\title{
Introducting short-stay intensive care after coronary artery surgery : studies in the development of a clinical pathway protocol
}

Citation for published version (APA):

Heijmans, J. H. (2007). Introducting short-stay intensive care after coronary artery surgery : studies in the development of a clinical pathway protocol. [Doctoral Thesis, Maastricht University]. Datawyse / Universitaire Pers Maastricht. https://doi.org/10.26481/dis.20071129jh

Document status and date:

Published: 01/01/2007

DOI:

10.26481/dis.20071129jh

Document Version:

Publisher's PDF, also known as Version of record

Please check the document version of this publication:

- A submitted manuscript is the version of the article upon submission and before peer-review. There can be important differences between the submitted version and the official published version of record.

People interested in the research are advised to contact the author for the final version of the publication, or visit the DOI to the publisher's website.

- The final author version and the galley proof are versions of the publication after peer review.

- The final published version features the final layout of the paper including the volume, issue and page numbers.

Link to publication

\footnotetext{
General rights rights.

- You may freely distribute the URL identifying the publication in the public portal. please follow below link for the End User Agreement:

www.umlib.nl/taverne-license

Take down policy

If you believe that this document breaches copyright please contact us at:

repository@maastrichtuniversity.nl

providing details and we will investigate your claim.
}

Copyright and moral rights for the publications made accessible in the public portal are retained by the authors and/or other copyright owners and it is a condition of accessing publications that users recognise and abide by the legal requirements associated with these

- Users may download and print one copy of any publication from the public portal for the purpose of private study or research.

- You may not further distribute the material or use it for any profit-making activity or commercial gain

If the publication is distributed under the terms of Article $25 \mathrm{fa}$ of the Dutch Copyright Act, indicated by the "Taverne" license above, 


\section{Introducing}

short-stay intensive care after coronary artery surgery

Studies in the development of a

clinical pathway protocol 
ISBN 9789052786766

(C) Copyright ].H. Heijmans, Maastricht 2007

Layout en druk: Datawyse / Universitaire Pers Maastricht Cover artwork: T. Heijmans - Debije 


\section{Introducing short-stay intensive care after coronary artery surgery}

Studies in the development of a clinical pathway protocol

\section{PROEFSCHRIFT}

ter verkrijging van de graad van doctor aan de Universiteit Maastricht, op gezag van de Rector Magnificus, Prof. mr. G.P.M.F. Mols volgens het besluit van het College van Decanen, in het openbaar te verdedigen op donderdag 29 november 2007 om 14.00 uutr

door

Joannes Hubertus Heijmans

Geboren te Echt

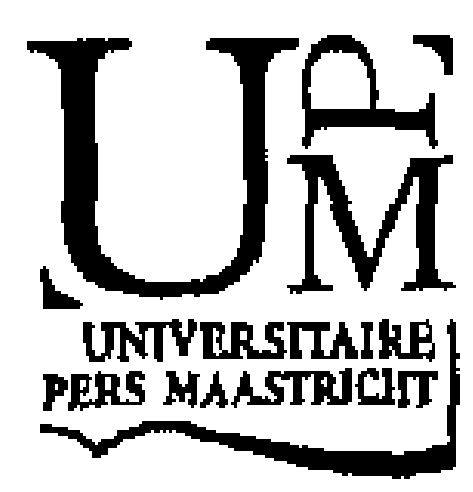




\section{PROMOTORES}

Prof. dr. M. van Kleef

Prof. dr. J.G. Maessen

\section{COPROMOTOR}

Dr. P.H.M.J. Roekaerts

\section{BEOORDELINGSCOMMISSIE}

Prof. dr. M.J.H.M. Jacobs (voorzitter)

Prof. dr. R. Autschbach (University of Aachen, Germany)

Prof. dr. A.P.M. Gorgels

Prof. dr. C.J. Kalkman (Universitair Medisch Centrum Utrecht)

Prof. dr. A. van Zundert (Catharina ziekenhuis, Eindhoven)

Prof. dr. J.H. Zwaveling 


\section{Content}

$\begin{array}{lll}\text { Chapter } 1 & \text { Introduction } & 7\end{array}$

Chapter 2 History of perioperative management in cardiac surgery

Chapter 3 Aims and outline of the thesis

Chapter 4 Risk stratification for adverse outcome in cardiac surgery

Chapter 5 Remifentanil provides better protection against noxious stimuli during cardiac surgery than alfentanil

Chapter 6 Comparison of the modulatory effects of four different fast-track anesthetic techniques on the inflammatory response to cardiac surgery with cardiopulmonary bypass

Chapter 7 Pulmonary function and inflammatory markers in patients undergoing coronary revascularization with or without cardiopulmonary bypass

Chapter 8 Assessment of cardiac responsiveness to fluid therapy after cardiac surgery

Chapter 9 Shortening ICU stay after coronary artery surgery

Chapter 10 Summary, discussion and conclusions

Chapter 11 Nederlandse samenvatting

Dankwoord

Curriculum Vitae

Publications 
CHAPTER 1

\author{
Introduction
}


Coronary artery bypass graft surgery, for the treatment of ischaemic heart disease, is a common procedure that is performed about 12500 times a year in the Netherlands. The procedure is complex, expensive and demanding for the hospital that provides this care. Because of the high costs involved and the high number of surgeries performed, coronary artery bypass graft surgery is classified as a high volume and high costs procedure. ${ }^{1,2}$

There is an increase in medical demand from the public community as a whole and there is an ongoing growth of the available medical technology and possibilities that are offered to the public community. ${ }^{3}$ These two factors added together impose an intolerable financial burden on the shoulders of the medical care providers and their governments. In order to prevent indefinite expenses in health care, governments are forced to contain costs and resources. Economics are forced into the decision making process, a process that used to belong to the medical doctor alone. ${ }^{+}$The benefit of a treatment must be put in perspective of the costs of that particular treatment. This is also a total new concept of medical decision making. The consequence of this is that the medical field has to change in the way it used to treat his patients and adapt to the new expectations and demands of the public and the government. The medical profession is expected to provide its patients with the highest standard of care, quality and safety of care, care that is available for everyone, that is efficient and efficacious and to do so with less means and reduced budgets.

A new concept, evidence-based medicine (EBM) was introduced in the early nineties. ${ }^{5} \mathrm{EBM}$ is "the integration of best research evidence with clinical expertise and patient values". "The purpose of EBM is to base medical practice, decision making, therapeutics, etc. on evidence-based clinical research findings eliminating intuition or unsystematic clinical experience. A purpose of EBM is to select and formulate what is considered 'best clinical practice', including economical aspects. ${ }^{7}$ The results from the conjunction of EBM with clinical expertise and patient values is then translated into hospital guidelines and protocols.

The medical philosophy on how to treat patients has changed from a medical specialist centred medical practice to a patient centred medical practice. The patient is no longer referred to a medical specialist and than referred to another and so on, instead the patient with his/her disease enters a disease-specific clinical pathway, for example cardiovascular, where different medical specialties (cardiology, cardiac surgery, anaesthesiology, ...) together are responsible for the care-process of the patient. ${ }^{8,9}$ Although there is no uniform definition of a clinical pathway, a clinical pathway can be described best by the definition of L. De Bleser as:" ... a method for the patient-care management of a well-defined group of patients during a welldefined period of time. A clinical pathway explicitly states the goals and key elements of care based on Evidence Based Medicine guidelines, best practice and patient expectations by facilitating the communication, coordinating roles and sequencing the activities of the multidisciplinary care team, patients and their rela- 
tives; by documenting, monitoring and evaluating variances; and by providing the necessary resources and outcomes. ${ }^{10}$ The aim of a clinical pathway is to improve the quality of care, reduce risks, increase patient satisfaction and increase the efficiency in the use of resources." This of course requires teamwork among the different medical specialties.

The present series of investigations was undertaken to provide sound information on issues which were thought to be essential in the development of an optimal clinical pathway protocol for selected low-risk coronary artery bypass graft surgery patients in the University Hospital in Maastricht. 


\section{REFERENCES}

1. Mark DB. Implications of Cost in Treatment Selection for Patients With Coronary Heart Disease. Annals of Thoracic Surgery 1996; 61: S12 - S15.

2. Birkmeyer JG, Skinner JS, and Wennberg DE. Will Volume-Based Referral Strategies Reduce Costs Or Just Save Lives? Health Affairs 2002;21:234- 241.

3. Mossialos E and Le Grand J (Editors). Health Care and Cost Containment in the European Union. Ashgate Publishing Limited, 1999. England.

4. Cheng DCH. Fast Track Cardiac Surgery Pathways. Early Extubation, Process of Care, and Cost Containment. Anesthesiology 1998; 88: 1429 - 1433.

5. Jamrozik K. Why Evidence-Based Medicine? Best Practice \& Research Clinical Anaesthesiology $2001 ; 15: 505-518$.

6. Sackett DL, Straus SE, Richardson WS, Rosenberg W, and Haynes RB. Evidence-Based Medicine. How to Practice and Teach EBM. Churchill Livingstone, second edition, 2000, London.

7. Pedersen T. Evidence-Based Anaesthesia and Health Economics. Best Practice \& Research Clinical Anaesthesia 2006; 20:347 - 360 .

8. Grol R and Wensing M (Editors). Implementatie. Effectieve Veranderingen in de Patientenzorg. Elsevier Gezondheidzorg, $2^{\text {de }}$ druk, 2001, Maarsen.

9. Kehlet $\mathrm{H}$ and Wilmore DW. Multimodal Strategies to Improve Surgical Outcome. The American Journal of Surgery 2002;183:630-641.

10. De Bleser L, Depreitere R, De Waele K, Vanhaecht K, Vlayen J, and Sermeus W. Defining Pathways. Journal of Nursing Management 2006; 14:553 - 563. 


\section{CHAP'TER 2}

History of perioperative management in cardiac surgery 
$1950-1990$

\section{Surgery}

The first surgical attempt to increase myocardial bloody supply, by creating a collateral circulation within the pericardium, was performed by C. Beck, Professor of Neurosurgery at the Western Reserve School of Medicine, Cleveland, USA in 1932.1

It lasted until 1950 when Arthur M. Vineberg at McGill University, Montreal, Canada operated on the first human patient. He mobilized the left internal mammary artery and implanted the end of the vessel into a tunnel in the left ventricle close to the left anterior descending coronary artery. ${ }^{\prime}$ So with Arthur $M$. Vineberg in 1950 the inception of an era of modern coronary artery bypass graft surgery began.

\section{Anaesthesia}

The anaesthetic technique in the early days of cardiac surgery was inhalationalbased. Oxygen/nitrous oxide in combination with volatile anaesthetics such as enflurane, halothane, or isoflurane were used. This anaesthetic technique however was associated with haemodynamic instabilities. For the treatment of hypo- and hypertension, additional pharmacological drugs had to be used. Another problem was the occurrence of awareness, meaning that some patients were apparently not deeply asleep at some timepoint during surgery and recalled after surgery some intraoperative events. ${ }^{2-4}$ Clinical observations showed that morphine did not cause cardiac depression and that the application of large doses of morphine did not have discernible haemodynamic effects. Lowenstein and co-workers demonstrated that a large dose of $3.0 \mathrm{mg} \mathrm{kg}^{-1}$ morphine-sulphate as an analgesic technique provided haemodynamic stability in cardiac surgery, even in patients with marginal cardiac reserve. ${ }^{5}$ The high-dose morphine-based anaesthetic technique seemed to be the technique of choice. However, the use of morphine as a mono-anaesthetic technique was not without side effects. Morphine produced profound analgesia but did not consistently caused loss of consciousness, so supplemental anaesthesia had to be given to provide hypnosis. Another problem with high-dose opioid anaesthesia was that the respiratory depression was prolonged into the postoperative period for $8-$ 12 hours which required controlled or assisted ventilation for a minimum of 12 to 18 hours. ${ }^{6,7}$ In 1978, Stanley and colleagues demonstrated the same absence of cardiovascular instabilities and attenuation of hormonal stress responses during anaesthesia using high doses of fentanyl, a new synthetic opioid at the time. ${ }^{8}$ The advantages of fentanyl as a sole anaesthetic over morphine-sulphate were numerous. Less hypotension associated with histamine release was observed, unconsciousness developed at relative low doses (without certaincy that amnaesia was achieved) and less prolonged respiratory depression was seen. ${ }^{\text {g-11 }}$ This lead to the worldwide 
acceptance of a high-dose opioid-based mono-anaesthetic technique as the technique of choice for cardiac anaesthesia. Very high doses of fentanyl up to $150 \mu \mathrm{g} \mathrm{kg}$ ${ }^{1}$ were then used to achieve suppression of hypertensive responses. ${ }^{12} \mathrm{De}$ Lange and colleagues, comparing the synthetic opioid sufentanil with fentanyl concluded that the more potent sufentanil was a superior narcotic in suppression of noxious stimuli as compared to fentanyl and that preoperative use of beta-adrenergic blocking drugs reduced intraoperative hypertension also. ${ }^{13}$ Another study by Sonntag and co-workers however, comparing different doses of sufentanil, concluded that sufentanil was not able to prevent hypertension associated with sternotomy or the occurrence of ischaemia and in this respect was not different from fentanyl. ${ }^{1+}$ As already stated, a consequence of the high-dose opioid-based mono-anaesthetic technique was the long postoperative period of respiratory depression and associated dependency of the patient on ventilatory support or assistance. However, at that time, it was thought that prolonged postoperative mechanical ventilation affected outcome beneficially. ${ }^{15-17}$ So far, the advantages of the high-dose opioid anaesthetic technique were appealing. However, no study had investigated the relationship between the choice of anaesthetic technique and outcome. In two studies, the relationship between the choice of the primary anaesthetic agent and postoperative outcome was investigated. The effects of several intravenous opioid-based and inhalational volatile-based anaesthetic techniques on the appearance of new myocardial ischaemia, postoperative myocardial infarction and/or death were studied. The conclusion of both studies was that none of the primary anaesthetics used influenced outcome. In both these studies $(n=1.012$ and $n=1.094)$, patients were ventilated overnight and stayed in the ICU for two to three days. ${ }^{18,19}$

\section{Perioperative management}

Perioperative management of these patients was more or less similar in most surgical institutions. The cardiac surgical patient was admitted to the hospital the day before the operation. After surgery under high-dose opioid anaesthesia, he was ventilated postoperatively for 24 hours or overnight with an average intensive care unit stay of 24-72 hours and a hospital length of stay of 12 days. ${ }^{20}$ The main reasons for prolonged mechanical ventilation were to reduce respiratory complications and to reduce the effort of breathing. ${ }^{15-17}$ The effort of spontaneous respiration was assumed to represent an extra burden to an already jeopardized myocardium. ${ }^{15}$

\section{Perioperative myocardial ischaemic damage}

A major concern in coronary artery surgery is the development of postoperative myocardial infarction, since this accounts for up to $40 \%$ of the mortality following CABG surgery. ${ }^{21}$ In a multicenter study, the Collaborative Study in Coronary Artery Surgery (CASS) involving 15 institutions and 6.176 patients on operative mortality in coronary artery surgery, an operative mortality of $2.3 \%$ was 
found. ${ }^{22}$ The incidence of perioperative $Q$-wave infarction was $4.6 \%$ and for the patients who did develop new postoperative $Q$ waves the mortality was $9.7 \%$ versus $1 \%$ in the patients who did not. Long-term survival was also adversely affected by the appearance of new postoperative Q-waves, with three-year survival rates of $85 \%$ versus $95 \%$ in patients with no new $Q$-wave infarction. ${ }^{23}$ The question raised then by Slogoff was: "Does perioperative myocardial ischemia lead to postoperative myocardial infarction?". Slogoff and Keats described in 1985 that the incidence of postoperative myocardial infarction was almost three times as high, $6.9 \%$ versus $2.5 \%$, in patients with perioperative ischaemic ST-segment depressions. In this study, ischaemia was significantly related to tachycardia but not to hypertension or hypotension and was frequently observed in the absence of haemodynamic instabilities. ${ }^{21}$ Knight and co-workers, investigating the relationship of pre-operative, intra-operative and post-operative ischaemia concluded that: 1) Coronary artery surgery patients have frequent pre-operative episodes of myocardial ischaemia, most of which are silent; 2) Anaesthesia and surgery do not worsen the preoperative ischaemic pattern; 3) ECG changes suggestive of nyocardial ischaemia frequently follow CABG surgery, although their pathogenesis and significance are yet unknown; 4) The majority of perioperative ischaemic ECG changes occur without acute haemodynamic changes prior to the onset of ischaemia; and 5) The preoperative ischaemic pattern appears to be recapitulated intraoperatively. ${ }^{2+}$ It is therefore relevant to examine the preoperative ischaemic pattern to assess the impact of anaesthesia and surgery on the development of intraoperative myocardial ischaemia. Because of the small study size and small number of postoperative myocardial infarctions, the researchers were unable to determine a relationship between perioperative ischaemia and postoperative myocardial infarction. Mangano and coworkers, investigating perioperative myocardial ischaemia in relation to cardiac morbidity and mortality in men undergoing non-cardiac surgery, found that postoperative myocardial ischaemia during the first 48 hours after surgery resulted in a nearly threefold increase in the odds of having an adverse cardiac outcome and a ninefold increase in the odds of having an ischaemic event such as cardiac death, nonfatal myocardial infarction, or unstable angina. They concluded that prevention of the occurrence and possible therapy of myocardial ischaemia in the postoperative period plays an important role in reducing perioperative morbidity. ${ }^{25}$ In a review about perioperative cardiac morbidity, the same author concluded that studies about postoperative predictors on outcome are lacking. ${ }^{26}$ In a prospective randomized study about postoperative myocardial ischaemia after $C A B G$ surgery, they concluded that the severity of ischaemic episodes can be diminished by use of prolonged intensive analgesia. ${ }^{27}$ Scientific proof that postoperative myocardial ischaemia is related to bad outcome and can be prevented by prolonged mechanical ventilation under analgo-sedation was in support for using the high-dose opioidbased mono-anaesthetic technique in CABG surgery, which inherently results in a 
prolonged postoperative period of deep analgesia and deep sedation necessitating mechanical ventilation.

\section{$1990-2007$}

Perioperative management of the coronary artery surgery patients changed dramatically over the last three decades and was associated with significantly reduced length of in-hospital stay. Several factors made fast-tracking of CABG patients not only necessary, but also feasible. These factors will be discussed in brief in the next paragraph.

\section{Changing insights on perioperative myocardial ischaemic complications}

The routine use of prolonged mechanical ventilation and its supposed beneficial effects was already questioned in the late seventies, especially in patients after uncomplicated cardiac surgery. Some authors thought that selected cardiac surgery patients with an uncomplicated operative and postoperative course would benefit from the undesirable effects of mechanical ventilation such as pulmonary infection, life-threatening airway obstruction, discomfort, and the need for heavy sedation. Prakash showed in 1977 that early extubation within 3 hours after chest closure, was successful in 118 out of 142 cardiac surgery patients. ${ }^{28}$ Quasha and co-workers investigated whether the duration of postoperative endotracheal intubation affected cardiopulmonary morbidity, haemodynamic performance, patient stress, drug utilization, or rate of recovery in the intensive care unit. No differences were found between those patients who were extubated early after 2 hours versus those who were extubated late after 18 hours, except that the early extubated patients received less morphine and diazepam. They concluded that early extubation is safe and does not increase postoperative cardiac or pulmonary complications, although ICU length of stay was the same for both groups (46 and 57 hours) ${ }^{29}$ However, the real scientific debate on pro and con early extubation started in the early nineties and is still ongoing. ${ }^{31,31}$ Justifications for the practice of prolonged ventilation were: (1) the presumed beneficial effect of high-dose opioid anaesthesia, which delayed weaning from artificial ventilation; (2) the frequent respiratory complications in the early postoperative phase; (3) the increased work of breathing in spontaneously breathing patients would represent an added burden on the myocardium at a time when cardiac function may still be compromized; (4) physiologic stress may induce myocardial ischaemia; (5) continuous intense postoperative analgo-sedation in combination with mechanical ventilation decreases myocardial ischaemic episodes in the early postoperative phase. ${ }^{31-34}$

New insights on the occurrence of perioperative myocardial ischaemic complications in coronary artery surgery did no longer supported the concept of beneficial prolonged mechanical ventilation under deep analgosedation. Indeed, 
perioperative myocardial ischaemia appeared to be related to myocardial oxygen/supply imbalances in less than $40 \%$ of the cases. Other aetiologic factors were considered to be even more important in the development of perioperative myocardial ischaemia. Vascular endothelial function, reperfusion injury, the systemic inflammatory response syndrome, coagulation abnormalities and vasomotor effects were found to be all critically important to outcome. Providing haemodynamic stability and maintaining the myocardial oxygen demand/supply ratio in balance can therefore no longer be considered as the only measures to prevent ischaemic myocardial damage. Optimizing endothelial function by nitroglycerin, nitric oxide, calcium channel-blockers, adenosine regulation agents and serine protease inhibitors may be even more essential to prevent perioperative ischaemic events. ${ }^{35,36}$

There is now mounting scientific evidence that early extubation is safe. Cheng studied in 1996 the morbidity of fast track surgery and found no increase in perioperative cardiac, respiratory, haemodynamic or sympathoadrenal morbidity. ${ }^{37}$ In this study, improved postoperative recovery resulted in reduced lengths of stay in the ICU and in the hospital: extubation time 4.1 versus $18.9 \mathrm{~h}, \mathrm{ICU}$ discharge 17.3 versus $25.6 \mathrm{~h}$, hospital discharge 5.7 versus 6.6 days. Reyes, examining low and moderate-risk patients in 1997, did not report increases in clinically important respiratory, cardiac, neurologic, renal, infectious complications or increase in number of reoperations or postoperative deaths. It was found that most low and moderate-risk patients, in whom cardiac operations under cardiopulmonary bypass are performed with opioid anaesthesia, can be extubated between 7 and $11 \mathrm{~h}$ after operation. ${ }^{38}$ Silbert concluded, from a prospective randomized trial in 1998, that early extubation is safe for primary outcome variables, incidence of reintubation, time in ICU, length of hospital stay and CK and CK-MB concentrations. ${ }^{34}$ In two large retrospective studies, comparing fast-track and non-fast-track patients, no differences were found for mediastinal or sternal infection, leg wound infection, operative mortality, late 1 to 24 month mortality and 30-day hospital readmission. ${ }^{40,41}$ A study comparing the effects of early versus late extubation on postoperative myocardial ischaemia, length of stay in ICU and hospital did not find significant differences between the two groups. ${ }^{42}$ Dumas investigated the effect of timing of extubation on cognitive functioning at day 3 and 5 , and 8 weeks postoperatively and found no differences between early and late extubation. ${ }^{4.3}$ These data were confirmed by Michalopoulos who showed that fast-track is safe compared to routine extubation with no differences in postoperative complications, reintubations, readmissions or mortality. ${ }^{44}$ Nicholson focused his research in 2002 on pulmonary function after early extubation, but did not find an increase in respiratory complications. ${ }^{45}$ Interestingly, Dowd and colleagues reported a very low incidence of awareness of $0.3 \%$ when low doses of opioids with short acting anaesthetics were used to facilitate early extubation in fast-track anaesthesia. ${ }^{\text {to }}$ In 1998 , Weintraub and co-workers reported a retrospective analysis of 12266 patients 
operated from 1988 to 1996 . Mortality rates decreased from $4.7 \%$ to $2.7 \%$, Q-wave infarction fell from $4.1 \%$ to $1.3 \%$, mean hospital costs decreased and length of stay after surgery decreased from 9.2 to 5.9 days. ${ }^{+7}$ Meade and Hawkes reported that early extubation was associated with less time spend in the ICU and in the hospital and was not different for mortality in intensive care, thirty day mortality, myocardial ischaemia or reintubation as compared to conventionally extubated patients. ${ }^{48,49}$ Time spent in ICU and hospital were significantly shorter. In 2003, a meta-analysis by Myles on safety and effectiveness of fast-track cardiac anaesthesia did not find evidence for increased mortality or morbidity. ${ }^{50}$

\section{Changing patient demands versus economic restraints}

At the beginning of the new century, several reports showed that the trend in coronary surgery over time had been towards operating on older patients with more comorbidities and increased predicted operative risk. Paone and colleagues, in a retrospective study comparing patients older than 70 years versus younger patients using a 5 -day postoperative pathway found a mortality rate of $5.5 \%$ versus $1.0 \%$ and a length of hospital stay of 7.9 versus 6.4 days in the older group as compared to the younger group. ${ }^{51}$ Cheng and co-workers found in his study that an age over 70 years was associated with longer times until extubation and ICU discharge and hospital length of stay. ${ }^{52}$ It appears logic that for older and sicker patients, perioperative care should be modified according to the specific needs of this patient population.

Together with the increase in demands for medical care by the public, cost containment and cost reduction became a major issue in health care. The medical profession was from then on expected to provide more care with less means and with the same or even better quality of care. ${ }^{53}$ The medical profession was faced with the enormous challenge to do more and better with less. Economic considerations became an intrinsic part of the medical decision making process, where formerly only medical considerations were part of the decision making process. Costs saving evaluation methods such as cost-benefit analysis, cost-utility analysis, cost-effectiveness analysis and cost-minimization analysis have entered the medical field to stay. ${ }^{5+}$ It is therefore not surprising that for economical reasons the cardiac surgical patient's track was reevaluated. It was assumed that, rapid extubation now being considered safe, fast-tracking of the cardiac surgical patients could decrease costs significantly. Therefore, studies on cost-saving were performed. These studies focused mainly on time spent in the ICU and hospital and on the development of cheaper post-surgical units, standard (nurse-led) operating procedures and protocols. ${ }^{55,56}$ Two studies comparing drugs and nursing costs in the ICU in an early versus a late extubation group, found a cost reduction of $25 \%$ in the early extubation group. ${ }^{57,58}$ Less expensive postoperative care facilities became under investigation and were described by Westaby in 1993 and later by Massey and Sirio. 
56,51,(11) The role of the ICU nurse in fast tracking of these patients became more prominent and Anderson in 1995 and Davies in 1997 described nurse-initiated extubation strategies. ${ }^{61,62}$ In order to streamline the fast track process, protocols for medical management, early extubation, anaesthetic technique and discharge criteria were developed and guidelines to allow faster recovery and earlier discharge from the ICU and hospital by Engelman in 1994-1996 and later by Coe. ${ }^{41,63,614}$

\section{Changing anaesthetic practice}

The lower doses of opioids used in the anaesthetic technique to shorten the postoperative intubation time mandate the addition of hypnotic agents in order to make sure that the patient is sufficiently sedated and has no recall of intraoperative events. The so-called balanced anaesthetic technique, with lower doses of opioids in combination with intravenous and/or volatile hypnotic agents, became the backbone of anaesthesia management. ${ }^{6.5}$ Many new drugs with faster onset times and shorter half-lifes were investigated in order to find the best possible combination of drugs to optimize the quality of anaesthesia and analgesia with the least cardiovascular side effects. ${ }^{6{ }^{6 i-72}}$ The technique should make early extubation possible, provide analgesia into the postoperative period and allow early mobilization. New volatile anaesthetics (isoflurane, sevoflurane and desflurane), hypnotics (midazolam and propofol) and opioids (alfentanil and remifentanil) became available in clinical practice. ${ }^{73,74}$ Volatile anaesthetics were often part of the balanced technique because of their presumed myocardial protective preconditioning effect. ${ }^{74,75}$ The shortacting hypnotic propofol was found to be superior to the benzodiazepine midazolam as part of a balanced anaesthetic technique as well as for postoperative sedation in this clinical setting. ${ }^{76-78}$ Several studies investigated the effects of the short-acting opioid alfentanil and ultra-short acting opioid remifentanil on extubation time, analgesia, haemodynamic stability and side effects in cardiac surgery. ${ }^{52,79,80}$ From all these studies, it can be concluded that a balanced anaesthetic technique using short-acting agents can provide good anaesthesia for cardiac surgery and allows for rapid extubation whenever indicated.

Total continuous intravenous anaesthesia, combining a short-acting opioid with a short-acting hypnotic, and administered with the newly developed volumetric infusion pumps, became a frequently used anaesthetic technique. ${ }^{81,82}$ Intravenous applied combinations of medications usually had a synergistic interaction, in order to achieve a similar effect the concentration of both drugs could be diminished. ${ }^{8,8+4}$ The depth of anaesthesia could be titrated more precisely and scheduled extubation could be performed more easily.

A further development was the introduction of the target-controlled infusion (TCI) techniques. ${ }^{79,86), 85}$ Anaesthetic drugs were titrated to the concentrations needed at effect site. Doses required to produce effective anaesthetic depth appeared to be less when using TCI, facilitating rapid extubation after surgery. Also, 
the application of the pharmacokinetic concept of context-sensitive time, the time required to produce a certain percentage change from the target concentration of an intravenous delivered drug, made scheduled extubation more feasible. ${ }^{83.86}$

The introduction of the bispectral index (BIS) as a new measurement of the depth of anaesthesia prevented awareness and on the same time monitored the dept of anaesthesia and therewith the minimal concentration of anaesthesia required, preventing pharmacological overdose to occur. ${ }^{87-89}$

In general surgery, the application of neuraxial blockade by thoracic epidural or spinal anaesthesia, in combination with a general anaesthetic approach was shown to have many beneficial effects. ${ }^{6}()_{1} \% 3$ The intraoperative reduction in anaesthetic drugs and the optimal pain relief were associated with rapid postsurgical extubation, early mobilization and less respiratory complications. ${ }^{94}$ Studies evaluating spinal and epidural techniques in combination with general anaesthesia in cardiac surgery were initiated in 1994." Most studies demonstrated good intraoperative haemodynamic stability and found faster extubation times and better postoperative pain relief in patients receiving neuraxial blockade for cardiac surgery. "The contribution of the addition of neuraxial blockade to general anaesthesia on clinical outcome is less easily defined. A meta-analysis by Rodgers and co-workers showed that mortality was significantly reduced in patients allocated to neuraxial blockade. ${ }^{\%}$ Neuraxial blockade also reduced the risk of deep vein thrombosis, pulmonary embolism, transfusion requirements, pneumonia, respiratory depression, myocardial infarction, and renal failure. However, these techniques are always associated with the risk of spinal cord injury and hematoma especially in anticoagulated patients. ${ }^{97-9 \%}$ Therefore, the debate whether or not neuraxial blockade should be applied routinely in cardiac surgery is still ongoing. Some authors described this neuraxial blockade as being safe in cardiac surgery when adherence was paid to a set of standard safety measures. ${ }^{1011}$ Others question the use of an epidural technique for safety reasons and argue the value of this technique in fast-track cardiac surgery, as rapid extubation after surgery can also be performed after anaesthesia without epidural blockade. ${ }^{1(11}$

\section{Changing surgical practice}

Cardiopulmonary bypass became more safe and less traumatic during the past decades. Membrane oxygenators, filters, bio-compatible materials and miniaturization are only some of the factors which improved safety. ${ }^{102-1105}$ Several reports in the literature demonstrated that moderate hypothermia $\left(32-34^{\circ} \mathrm{C}\right)$ was not associated with worse cerebral outcome as compared to deeper hypothermia $\left(28^{\circ} \mathrm{C}\right)$. There is now a trend towards surgery with $\mathrm{CPB}$ at normothermia, which obviously makes the initial postoperative course more uneventful. ${ }^{106-110}$ Less tissue trauma and a reduced pro-inflammatory effect were important factors in improving outcome after cardiac surgery. Surgical techniques also developed and coronary surgery 
without the use of cardiopulmonary bypass, so-called off-pump surgery, became popular. "I Off-pump surgery avoided the damaging effect of cardiopulmonary bypass. Other minimal invasive techniques and the use of robotics are continuously under development. ${ }^{112}$

All these surgical developments decrease the extend of the surgical trauma and are therefore expected to improve outcome. The reduction in stress response associated with these techniques may account for a smoother postoperative course.

\section{Where do we go from here}

In the early days of cardiac surgery, perioperative management was the same in every single patient. After surgery under high-dose opioid anaesthesia, the patient was ventilated postoperatively for 24 hours or overnight with an average intensive care unit stay of 24-72 hours and a hospital length of stay of 12 days. ${ }^{11}$

The developments described above show that patient categories have dramatically changed, and apart from younger and healthier adults also the older and sicker patients with major co-morbidities have become surgical candidates. ${ }^{+1.113}$ Also, anaesthetic and surgical techniques were refined and less traumatic procedures can now be applied, whenever indicated. ${ }^{47,113-116}$ Furthermore, it became more and more obvious that fast-tracking of cardiac surgical patients is safe and cost-effective $37,39,+2-45,+8-53,613,117-1211$ These developments raised the question that perhaps a certain category of patients would be better off when treated according to a fast-track protocol, while other patients perhaps still required a more prolonged perioperative in-hospital treatment. In other words, different types of perioperative care should be defined according to the patient's needs. ${ }^{55,56,59}$

The first question raised was then how to select patients which are suitable for fast-tracking. ${ }^{121}$ Several risk stratification studies tried to describe preoperative factors which could divide patients into a low-risk group, suitable for fast-track care and a high-risk group, not suitable for fast-tracking. ${ }^{122-124}$ The preoperative factors associated with prolonged stay in the intensive care are numerous. ${ }^{51,121,125-131}$ The most frequently cited are older patients, female sex, preoperative use of diuretics, unstable angina, urgent intervention, preoperative intra-aortic balloon counterpulsation, peripheral vascular disease, diabetes mellitus, congestive heart failure, symptomatic vascular disease, chronic obstructive pulmonary disease, ambulatory difficulties, cognitive impairement, low left ventricle ejection fraction, preoperative creatinine level, low body surface area, preoperative haematocrit, and nonelective surgery. Several scoring systems were developed to guide the physician in his patient selection. ${ }^{122-124}$ Intra operative risk factors were also defined and included bypass time, blood transfusion, use of inotropic agents, duration of the surgical procedure, lower temperature on bypass, blood transfusion, and inotropic use. $^{132-134}$ Evidently, postoperative developing instabilities, e.g. haemodynamic 
destabilizing rhythm disturbances, may necessitate prolonged ICU and hospital stay. ${ }^{1.35} \cdot 1.38$

Once selection of patients eligible for fast-tracking was done, the second question was how to provide best clinical practice to these patients according to the principles of evidence-based medicine. ${ }^{139}$ The concept of evidence based medicine (EBM) was introduced in the nineties as a means to base medical decision making on scientific proof rather than on subjective interpretations. ${ }^{1+1}$ Another purpose of EBM was an economic one, to get rid of expensive unproven therapeutic options. ${ }^{1+1}$ It became evident that clinical pathway protocols, based on the EBMprinciples, were an indispensable tool in the practice of best medical management of several different categories of patients in various clinical settings.

In developing a clinical pathway protocol for fast-track cardiac surgery in lowrisk coronary artery surgery patients in the University Hospital in Maastricht, we encountered several problems for which the literature provided no answers. To answer these questions, we formulated several research questions and initiated several clinical trials in order to develop a clinical pathway protocol for these patients that was safe, cost-effective and evidence-based. 


\section{REFERENCES}

1. Westaby S and Bosher C. Landmarks in Cardiac Surgery. Isis Medical Media Ltd. Oxford, UK, 2000.

2. Conahan III TJ, Ominsky AJ, Wollman H, and Stroth RA. A Prospective Random Comparison of Halothane and Morphine for Open-Heart Anesthesia: One Year's Experience. Anesthesiology $1973 ; 38: 528-535$.

3. Hall GM, Young $C$, Holdcroft $A$, and Alaghband-Zadeh J. Substrate Mobilization During Surgery. A Comparison between Halothane and Fentanyl Anaesthesia. Anaesthesia 1978;33:924 -930 .

4. Hess W, Arnold B, Schulte-Sasse U, and Tarnow J. Comparison of Isoflurane and Halothane when used to Control Intraoperative Hypertension in Patients Undergoing Coronary Artery Bypass Surgery. Anesthesia \& Analgesia 1983;62:15 - 20.

5. Lowenstein E, Hallowell P, Levine FH, Daggett WM, Austen WG, and Laver MB. Cardiovascular Response to Large Doses Intravenous Morphine in Man. The New England Journal of Medicine $1969 ; 281: 1389-1393$.

6. Lowenstein E. Morphine "Anesthesia" - a Perspective. Anesthesiology 1971;35:563 - 565.

7. Arens JF, Benbow BP, Ochsner JL, and Theard R. Morphine Anesthesia for Aortocoronary Bypass Procedures. Anesthesia \& Analgesia 1972; 51: $901-909$.

8. Stanley TH, and Webster LR. Anesthetic Requirements and Cardiovascular Effects of FentanylOxygen and Fentanyl-Diazepan1-Oxygen Anesthesia in Man. Anesthesia \& Analgesia 1978; 57 : $411-416$.

9. Lunn JK, Stanley TH, Eisele J, Webster L, and Woodward A. High Dose Fentanyl Anesthesia for Coronary Artery Surgery: Plasma Fentanyl Concentrations and Influence of Nitrous Oxide on Cardiovascular Responses. Anesthesia \& Analgesia 1979; 58:390 - 395.

10. Waller JL, Hug Jr CC, Nagle DM, and Craver JM. Hemodynamic Changes during FentanylOxygen Anesthesia for Aortocoronary Bypass Operation. Anesthesiology 1981; 55:212 - 217.

11. Stanley TH, Berman L, Green $O$, and Robertson D. Plasma Catecholamine and Cortisol Responses to Fentanyl-Oxygen Anesthesia for Coronary-Artery Operations. Anaesthesiology $1980 ; 53: 250-253$.

12. Wynands JE, Townsend GE, Wong P, Whalley DG, Srikant CB, and Patel YC. Blood Pressure Response and Plasma Fentanyl Concentrations during High- and Very High-Dose Fentanyl Anesthesia for Coronary Artery Surgey. Anesthesia \& Analgesia 1983; 62:661 - 665.

13. De Lange S, Boscoe MJ, Stanley TH, and Pace N. Comparison of Sufentanil-O2 and FentanylO2 for Coronary Artery Surgery. Anesthesiology 1982;56:112 - 118 .

14. Somntag $H$, Stephan $H$, Lange $H$, Rieke $H$, Kettler D, and N. Martschausky N. Sufentanil Does Not Block Sympathetic Responses to Surgical Stimuli in Patients Having Coronary Artery Revascularisation Surgery. Anesthesia \& Analgesia 1989; 68:584-592.

15. Thung N, Herzog P, Christlieb I, Thompson Jr WM, and Dammann JF. The Cost of Respiratory Effort in Postoperative Cardiac Patients. Circulation 1963; 28:552 - 559.

16. Lefemine AA and Harken DE. Postoperative Care following Open-Heart Operations: Routine Use of Controlled Ventilation. Journal of Thoracic and Cardiovascular Surgery 1966; 52: 207 216.

17. Andersen NB and Ghia J. Pulmonary Function, Cardiac Status, and Postoperative Course in Relation to Cardiopulmonary Bypass. The Journal of Thoracic and Cardiovascular Surgery 1970; $59: 474-483$.

18. Slogoff S and Keats AS. Randomized Trial of Primary Anesthetic Agents on Outcome of Coronary Artery Bypass Operations. Anesthesiology 1989;70:179-188.

19. Tuman KJ, McCarthy RJ, Spiess BD, DaValle M, Dabir R, and Ivankovich AD. Does Choise of Anesthetic Agent Significantly Affect Outcome after Coronary Artery Surgery? Anesthesiology 1989; 70: 189 - 198 . 
20. Klineberg PL, Geer RT, Hirsh RA, and Aukburg SJ. Early Extubation After Coronary Artery Bypass Graft Surgery. Critical Care Medicine 1977; 5: 272 - 274.

21. Slogoff S and Keats AS. Does Perioperative Myocardial Ischemia Lead to Postoperative Myocardial Infarction? Anesthesiology 1985; 62: 107- 114.

22. Kennedy JW, Kaiser GC, Fisher LD, Maynard C, Fritz JK, Myers W, Mudd JG, Ryan TJ, and Coggin J. Multivariate Discriminant Analysis of the Clinical and Angiographic Predictors of Operative Mortality from the Collaborative Study in Coronary Artery Surgery (CASS). Journal of Thoracic and Cardiovascular Surgery 1980;80:876-887.

23. Chaitman BR, Alderman EL, Sheffield LT, Tong T, Fisher L, Mock MB, Weins RD, Kaiser GC, Roitman D, Berger R, Gersh B, Schaff $H$, Bourassa MG, Killip T, and Participating CASS Medical Centers. Use of Survival Analysis to Determine the Clinical Significance of New QWaves After Coronary Bypass Surgery. Circulation 1983; 67:302 - 309.

24. Knight AA, Hollenberg M, London MJ, Tubau J, Verier E, Browner W, Mangano DT, and The S.P.I. Research Group. Perioperative Myocardial Ischemia: Importance of the Preoperative Ischemic Pattern. Anesthesiology 1988; 68:681 - 688.

25. Mangano DT, Browner WS, Hollenberg M, London MJ, Tubau JF, Tateo IM, and The Study of Perioperative Ischemia Research Group. Association of Perioperative Myocardial Ischemia with Cardiac Morbidity and Mortality in Men Undergoing Noncardiac Surgery. The New England Journal of Medicine 1990; 323: 1781 - 1788.

26. Mangano DT. Perioperative Cardiac Morbidity. Anesthesiology 1990; 72: $153-184$.

27. Mangano DT, Siliciano D, Hollenberg M, Leung JM, Browner WS, Goehner P, Merrick S, Verrier E, and The Study of Perioperative Ischemia (SPI) Research Group. Postoperative Myocardial Ischemia. Therapeutic Trials Using Intensive Analgesia Following Surgery. Anesthesiology 1992; 342: $342-353$.

28. Prakash O, Jonson B, Meij S, Bos E, Hugenholtz PG, Nauta J, and Hekman W. Criteria for Early Extubation After Intracardiac Surgery in Adults. Anesthesia \& Analgesia 1977; 56: 703 - 708.

29. Quasha AL, Locber N, Feeley TW, Ullyot DJ, and Roizen MF, Postoperative Respiratory Care: A Controlled Trial of Early and Late Extubation following Coronary-Artery Bypass Grafting. Anesthesiology 1980;52: 135 - 141.

30. Higgins TL. Pro: Early Endotracheal Extubation is Preferable to Late Extubation in Patients following Coronary Artery Surgery. Journal of Cardiothoracic and Vascular Anesthesia 1992; 6: $488-493$.

31. Siliciano D. Con: Early Extubation is Not Preferable to Late Extubation in Patients Undergoing Coronary Artery Surgery. Journal of Cardiothoracic and Vascular Ancsthesia 1992;6:494 - 498.

32. Higgins TL. Safety Issues Regarding Early Extubation After Coronary Artery Bypass Surgery. Journal of Cardiothorac and Vascular Anesthesia 1995; 9:24-29.

33. Mangano DT. Biventricular Function after Myocardial Revascularization in Humans: Deteriorating and Recovery Patterns during the First 24 Hours. Anesthesiology 1985;62:571 - 577.

34. Breisblatt WM, Stein KL, Wolfe CJ, Follansbee WP, Capozzi J, Armitage JM, and Hardesty RL. Acute Myocardial Dysfunction and Recovery: A Common Occurrence After Coronary Bypass Surgery. Journal of the American College of Cardiology 1990; 15: $1261-1269$.

35. Verrier ED, Wright IH, Cochran RP, and Spiess BD. Changes in Cardiovascular Surgical Approaches to Achieve Early Extubation. Journal of Cardiothoracic and Vascular Anesthesia 1995; 9, Suppl 1:10-15.

36. Hansson GK. Mechanisns of Disease. Inflammation, Atherosclerosis, and Coronary Artery Disease. The New England Journal of Medicine 2005; 352: 1685 - 1695.

37. Cheng DCH, Karski J, Peniston C, Asokumar B, Raveendran G, Cartoll J, Nierenberg H, Roger S, Mickle D, Tong J, Zelovitsky J, David T, and Sandler A. Morbidity Outcome in Early versus Conventional Tracheal Extubation after Coronary Artery Bypass Grafting: A Prospective Randomized Controlled Trial. Journal of Thoracic and Cardiovascular Surgery 1996;112:755 764. 
38. Reyes A, Vega G, Blancas R, Morato B, Moreno J-L, Torrecilla C, and Cereijo E. Early vs Conventional Extubation After Cardiac Surgery With Cardiopulnonary Bypass. Chest 1997; 112: $193-201$.

39. Silbert BS, Santamaria JD, O\&Brien JL, Blyth CM, Kelly WJ, Molnar RR, and the Fast Track Cardiac Care Team. Early Extubation Following Coronary Artery Bypass Surgery. A Prospective Randomized Controlled Trial. Chest 1998; 113: 1481 - 1488.

40. Engelman RM, Rousou JA, Flack III JE, Deaton DW, Humphrey CB, Ellison LH, Allmendinger PD, Owen SG and Pekow PS. Fast-Track Reovery of the Coronary Bypass Patient. Annals of Thoracic Surgery 1994;58:1742 - 1746.

41. Lee JH, Graber R, Popple CG, Furey E, Lyons T, Murrell HK, and Geha AS. Saftey and Efficacy of Early Extubation of Eldery Coronary Artery Bypass Surgery Patients. Journal of Cardiothoracic and Vascular Anesthesia 1998;12:381 - 384.

42. Berry PD, Thomas SD, Mahon SP, Jackson M, Fox MA, Fabri B, Weir WI, and Russell GN. Myocardial Ischaemia After Coronary Artery Bypass Grafting: Early vs Late Extubation. British Journal of Anaesthesia 1998; 80:20 - 25.

43. Dumas A, Gilles GH, Searle N, and Cartier R. Early Versus Late Extubation After Coronary Artery Bypass Grafting; Effects in Cognitive Function, Journal of Cardiothoracic and Vascular Anesthesia 1999; 13:130 - 135.

44. Michalopoulos A, Nikolaides A, Antzaka C, Deliyanni M, Smirli A, Geroulanos S, and Papadimitriou L. Change in Anaesthesia Practice and Postoperative Sedation Shortens ICU and Hospital Length of Stay Following Coronary Artery Bypass Surgery. Respiratory Medicine 1998; 92: $1066-1070$.

45. Nicholson DJ, Kowalski SE, Hamilton A, Meyers MP, Serrette C, and Duke PC. Postoperative Pulmotary Function in Coronary Artery Bypass Graft Surgery Patients Undergoing Early Tracheal Extubation: A Comparison Between Short-Term Mechanical Ventilation and Early Extubation. Journal of Cardiothoracic and Vascular Anesthesia 2002;16:27 - 31 .

46. Dowd NP, Cheng DCH, Karski JM, Wong DT, Carroll Munro JA, Sandler AN, Intraoperative Awareness in Fast-track Cardiac Anesthesia. Anesthesiology 1998; 89: 1068 - 1073.

47. Weintraub WS, Craver JM, Jones E, Gott JP, Deaton C, Culler SD and Guyton RA. Improving Cost and Outcome of Coronary Surgery. Circulation 1998; 98: II-23-II-28.

48. Meade $M O$, Guyatt $G$, Butler R, Elnıs B, Hand L, Ingram A, and Griffith L. Trials Comparing Early vs Late Extubation Following Cardiovascular surgery. Chest 2001 (Supplement); 120: $445 \mathrm{~S}$ $-452 S$

49. Hawkes, CA, Dhileepan S, and Foxcroft D. Early Extubation for Adult Cardiac Surgical Patietrus (Review). Cochrane Database of Systematic Reviews. 2003; Issue 4. Art. No.: CD003587: 1-29.

50. Myles PS, Daly DJ, Djaiani G, Lee A, and Cheng DCH. A Systematic Review of the Safety and Effectiveness of Fast-track Cardiac Anesthesia. Anesthesiology 2003; 99:982 - 987.

51. Paone G, Higgins RSD, Havstad SL, and Silverman NA. Does Age Limit the Effectiveness of Clinical Pathways After Coronary Artery Bypass Graft Surgery? Circulation 1998; 98: 11-41-I145.

52. Cheng DCH, Newman MF, Duke P, Wong DT, Finegan B, Howie M, Fitch J, Bowdle A, Hogue $C$, Hillel Z, Pierce E, and Bukenya D. The Efficacy and Resource Utilization of Remifentanil and Fentanyl in Fast-Track Coronary Artery Bypass Graft Surgery: A Prospective Randomized, Double-Blinded Controlled, Multi-Center Trial. Anesthesia \& Analgesia 2001;92:1094-1102.

53. Cheng DCH. Fast-Track Cardiac Surgery: Economic Implications in Postoperative Care. Journal of Cardiothoracic and Vascular Anesthesia 1998; 12:72 - 79.

54. Velasco FT, Tarlow LS, and Thomas SJ. Economic Rational for Early Extubation. Journal of Cardiothoracic and Vascular Anesthesia 1995; 9, Suppl 1:2 - 9.

55. Chong JL, Grebenik C, Sinclair M, Fisher A, Pillai R, and Westaby S. The Effect of a Cardiac Surgical Recovery Area on the Timing of Extubation. Journal of Cardiothoracic and Vascular Anesthesia 1993; 7: 137 - 141. 
56. Westaby S, Pillai R, Parry A, O'Regan D, Giannopoulos N, Grebenik K, Sinclair M, and Fisher A. Does Modern Cardiac Surgery Require Conventional Intensive Care? European Journal of Cardio-thoracic Surgery 1993; 7: 313-318.

57. Cheng DCH, Karski J, Peniston C, Raveendran G, Asokumar B, Carroll J, David T, and Sandler A. Early Tracheal Extubation After Coronary Artery Bypass Graft Surgery Reduces Costs and Improves Resourse Use. A Prospective Randomized Controlled Trial. Anesthesiology 1996; $85: 1300-1310$.

58. Sherry KM, McNamara J, Brown JS, and Drummond M. An Economic Evaluation of Propofol/Fentanyl Compared with Midazolam/Fentanyl on Recovery in the ICU following Cardiac Surgery. Anaesthesia 1996; 51:312 - 317.

59. Masscy D and Meggit G. Recovery Units: The Future of Postoperative Cardiac Care. Intensive and Clinical Care Nursing 1994; 10:71 - 74 .

60. Sirio CA and Martich GD. Who Goes to the ICU Postoperatively? Chest 1999;115:125S-129S.

61. Anderson J and O'Brien M. Challenges for the Future: The Nurse's Role in Weaning Patients from Mechanical Ventilation. Intensive and Critical Care Nursing 1995; 1:2 - 5 .

62. Davies N. Nurse-Initiated Extubation Following Cardiac Surgery. Intensive and Critical Care Nursing 1997; 13:77-79.

63. Engelman RM. Mechanisms to Reduce Hospital Stays. Annals of Thoracic Surgery, 1996; 61: $\mathrm{S} 26-\mathrm{S} 29$.

64. Coe V. Early Extubation: Perspective From a Community Hospital. Joumal of Cardiothoracic and Vascular Anesthesia 1995; 9, Suppl 1:37-43.

65. Karski JM. Practical Aspects of Early Extubation in Cardiac Surgery. Journal of Cardiothoracic and Vascular Anesthesia 1995; 9, Suppl 1:30-33.

66. Butterworth J, James R, Prielipp RC, Cerese J, Livingston J, and Burnett DA, the CABG Clinical Benchmarking Data Base Participants. Do Shorter-Acting Neuromuscular Blocking Drugs or Opioids Associate with Reduced Intensive Care Unit or Hospital Lengths of Stay After Coronary Artery Bypass Grafting? Anesthesiology 1998; 88: 1437-1446.

67. Ahonen J, Olkkola KT, Hynynen M, Seppala T, Ikavalko H, Remmerie B, and SaImenpera $M$. Comparison of Alfentanil, Fentanyl and Sufentanil for Total Intravenous Anaesthesia with Propofol in Patients Undergoing Coronary Artery Bypass Surgery. British Journal of Anaesthesia 2000; 85: $533-540$.

68. Ahonen J, Olkkola K T, Verkkala K, Heikkinen L, Jarvinen A, and Salmenpera M. A Comparison of Remifentanil and Alfentanil for Use with Propofol in Patients Undergoing Minimally Invasive Coronary Artey Bypass Surgery. Anesthesia \& Analgesia 2000; 90:1269 - 1274.

69. Mollhoff T, Herregods L, Moerman A, Blake D, MacAdams C, Demeyere R, Kirno K, Dybvik T, Shaikh S, and the Remifentanil Study Group. Comparative Efficacy and Saftey of Remifentanil and Fentanyl in "Fast-Track" Coronary Artery Bypass Graft Surgery: A Randonized, Doubleblind Study. British Journal of Anaesthesia 2001; 87: 718 - 726.

70. Engoren M, Luther G, and Fenm-Buderer N. A Comparison of Fentanyl, Sufentanil, and Remifentanil for Fast-Track Cardiac Anesthesia. Anesthesia \& Analgesia 2001; 93: 859- 864 .

71. Howie MB, Cheng D, Newman MF, Pierce ET, Hogue C, Hillel Z, Bowdle TA, and Bukenya D. A Randomized Double-Blinded Multicenter Comparison of Remifentanil Versus Fentanyl When Combined with Isoflurane/Propofol for Early Extubation in Coronary Artery Bypass Graft Surgery. Anesthesia \& Analgesia 2001; 92: 1084 - 1093.

72. Tritapepe L, Voci P, Di Giovanni C, Pizzuto F, Cuscianna E, Caretta Q, and Pietropaoli P. Alfentanil and Sufentanil in Fast-Track Anesthesia for Coronary Artery Bypass Graft Surgery. Journal of Cardiothoracic and Vascular Anesthesia 2002;16:157 - 162.

73. Engoren MC, Kraras C, and Garzia F. Propofol-Based versus Fentanyl-Isoflurane-Based Anesthesia for Cardiac Surgery.Journal of Cardiothoracic and Vascular Anesthesia 1998;12:177-181. 
74. De Hert SG, ten Broeke PW, Mertens E, Van Sommeren EW, De Blier I, Stockman BA, and Rodrigus IE. Sevolurane but Not Propofol Preserves Myocardial Function in Coronary Surgery Patients. Anesthesiology, 2002;97: 442 - 49.

75. Julier K, da Silva R, Garcia C, Bestmann L, Frascarolo P, Zollinger A, Chassot P-G, Schmid ER, Turina MI, von Segesser LK, Pasch T, Spahn DR, and Zaugg M. Preconditioning by Sevoflurane Decreases Biochemical Markers for Myocardial and Renal Dysfunction in Coronary Artery Bypass Graft Surgery: A Double--blinded, Placebo-controlled, Multicenter Study. Anesthesiology 2003; 98: 1315 - 1327.

76. Roekaerts PMHJ, Huygen FJPM, and de Lange S. Infusion of Propofol versus Midazolan for Sedation in the Intensive Care Unit following Coronary Artery Surgery. Journal of Cardiothoracic and Vascular Anesthesia 1993; 7:142 - 147.

77. Newman M and Reves JG. Midazolam is the Sedative of Choice to Supplement Narcotic Anesthesia. Pro: Midazolam Is the Sedative of Choise to Supplement Narcotic Anesthesia. Journal of Cardiothoracic and Vascular Anesthesia 1993; 7:615 - 619.

78. Roekaerts $P$ and de Lange S. Con: Midazolam is Not the Sedative of Choise to Supplement Narcotic Anesthesia. Journal of Cardiothoracic and Vascular Ansethesia 1993; 7:620 - 623.

79. Olivier P, Sirieix D, Dassier P, D'Attellis $\mathrm{N}$, and Baron J-F. Continuous Infusion of Remifentanil and Target-Controlled Infusion of Propofol for Patients Undergoing Cardiac Surgery: A New Approach for Scheduled Early Extubation. Journal of Cardiothoracic and Vascular Anesthesia $2000 ; 14: 29-35$.

80. De Castro V, Godet G, Mencia G, Raux M, and Coriat P.Target-Cotrolled Infusion for Remifentanil in Vascular Patients Improves Hemodynamics and Decreases Remifentanil Requirement. Anesthesia \& Analgesia 2003;96:33 - 38.

81. Manara AR, Monk CR, Bolsin SN, and Prys-Roberts C. Total I.V. Anaesthesia with Propofol and Alfentanil for Coronary Artery Bypass Grafting. British Journal of Anaesthesia 1991;66:716 -718 .

82. Bacon R, Chandrasekan V, Haigh A, Royston BD, Royston D, and Sundt T. Early Extubation After Open-Heart Surgery with Total Intravenous Anaesthetic Technique. The Lancet 1994; 345: $133-134$.

83. Glass PSA. Pharmacokinetic and Pharmacodynamic Principles in Providing "Fast-Track" Recovery. Journal of Cardiothoracic and Vascular Anesthetesia 1995; 9, Suppl 1:16- 20.

84. Short TG, Plummer JL, and Chui PT. Hypnotic and Anaesthetic Interactions Between Midazolam, Propofol and Alfentanil. British Journal of anaesthesia 1992; 69: 162 - 167.

85. Gale T, Leslie K, and Kluger M. Propofol Anaesthesia via Target Controlled Infusion or Manually Controlled Infusion: Effects on the Bispectral Index as a Measure of Anaesthesia Depth. Anaesthesia and Intensive Care 2001; 29:579-584.

86. Hughes MA, Glass PSA, and Jacobs JR. Context-Sensitive Half-Time in Multicompartment Pharmacokinetic Models for Intravenous Anesthetic Drugs. Anesthesiology 1992; 76:334-341.

87. Liu J, Singh H, and White PE. Electroencephalographic Bispectral Index Correlates with Intraoperative Recall and Depth of Propofol-Induced Sedation. Anesthesia \& Analgesia 1996; 84: $185-189$.

88. Puri GD and Murthy SS. Bispectral Index Monitoring in Patients Undergoing Cardiac Surgery under Cardiopulmonary Bypass. European Journal of Anaesthesiology 2003; 20:451 - 456.

89. Myles PS, Leslie K, McNeil J, Forbes A, Chan MTV, for the B-Aware Trial Group. Bispectral Index Monitoring to Prevent Awareness During Anacsthesia: The B-Aware Randomised Controlled Trial. The Lancet 2004; 363: 1757 - 1763.

90. Swenson JD, Hullander RM, Wingler K, and Leivers D. Early Extubation After Cardiac Surgery Using Combined Intrathecal Sufentanil and Morphine. Journal of Cardiothorac and Vascular Anesthesia 1994; 8:509-514. 
91. Shroff A, Rooke GA, and Bishop MJ. Effects of Intrathecal Opioid on Extubation Time, Analgesia, and Intensive Care Unit Stay Following Coronary Artery Bypass Grafting. Journal of Clinical Anesthesia 1997; 9:415 - 419.

92. Zarate E, Latham P, White PF, Bossard R, Morse L, Douning LK, Shi C, and Chi L. Fast-Track Cardiac Anesthesia: Use of Remifentanil Combined with Intrathecal Morphine as an Altenative to Sufentanil During Desflurane Anesthesia. Anesthesia \& Analgesia 2000;91:283 - 287.

93. Latham P, Zarate E, White PF, Bossard R, Shi C, Morse LS, Douning LK, and Chi L. Fast-Track Cardiac Anesthesia: A Comparison of Remifentanil Plus Intrathecal Morphine With Sufentanil in a Desflurane-Base Anesthetic. Journal of Cardiothoracic and Vascular Anesthesia 2000;14: p $645-651$.

94. Bettex DA, Schmidlin D, Chassot P-G, and Schmid ER. Intrathecal Sufentanil-Morphine Shortens the Duration of Intubation and Improves Analgesia in Fast-Track Cardiac Surgery. Canadian Journal of Anesthesia 2003; 49: 711 - 717.

95. Royse C, Royse A, Soeding P, Blake D, and Pang J. Prospective Randomized Trial of High Thoracic Epidural Analgesia for Coronary Artery Bypass Surgery. Annals of Thoracic Surgery 2003; 75:93 - 100 .

96. Rodgers A, Walker N, Schug S, McKee A, Kehlet H, van Zundert A, Sage D, Futter M, Saville G, Clark T, and MacMahon S. Reduction of Postoperative Mortality and Morbidity with Epidural or Spinal anaesthesia: Results from Overview of Randomised Trials. The British Medical Journal $2000 ; 321: 1-12$.

97. Liu S, Carpenter RL, and Neal JM. Epidural Anesthesia and Analgesia. Their Role in Postoperative Outcome. Anesthesiology 1995; 82: 1474 - 1506.

98. Goldstein S, Dean D, Kim SJ, Cocozello K, Grofsik J, Silver P, and Cody RP. A Survey of Spinal and Epidural Techniques in Adult Cardiac Surgery. Journal Cardiothoracic and Vascular Anesthesia $2001 ; 15: 158-168$.

99. GravleeGP. Epidural Analgesia and Coronary Artery Bypass Grafting: The Controversy Continues. Journal of Cardiothoracic and Vascular Anesthesia 2003; 17: $151-153$.

100. Pastor MC, Sanchez MJ, Casas MA, Mateu J, and Bataller ML. Thoracic Epidural Analgesia in Coronary Artery Bypass Graft Surgery: Seven Year's Experience. Journal of Cardiothoracic and Vascular Anesthesia 2003; 17: 154-159.

101. Walji S, Peterson RJ, Neis P, Dubroff R, Gray WA, and Benge W. Ultra-Fast Track Hospital Discharge Using Conventional Cardiac Surgical Techniques. Annals of Thoracic Surgery 1999; 67: 363 - 370 .

102. Nilsson L, Tyden $H$, Johnasson $O$, Nilsson $U$, Ronquist $G$, Venge P, Aberg T, and Nystrom SO. Bubble and Membrane OxygenatorsComparison of Postoperative Organ Dysfunction with Special Reference to Inflammatory Activity. Scandinavian Journal of Thoracic \& Cardiovascular Surgery 1990;24:59-64.

103. Moen O, Hogasen K, Fosse E, Dregelid E, Brockmeier V, Venge P, Harboe M, and Mollnes TE. Attenuation of Changes in Leucocyte Surface Markers and Complement Activation With Heparin-Coated Cardiopulmonary Bypass. Annals of Thoracic Surgery 1997; 63: 105 - 111.

104. Parolari A, Alamanni F, Naliato M, Spirito R, Franze V, Pompilio G, Agrifoglio M, and Biglioli. Adult Cardiac Surgery Outcomes: Role of the Pump Type. European Journal of CardioThoracic Surgery 2000; $18: 575-582$.

105. Rubens FD and Mesana T. The Inflammatory Response to Cardiopulmonary Bypass: A Therapeutic Overview. Perfusion 2004; 19: S5 - S12.

106. Christenson JT, Maurice J, Simonet FA, Velebit V, and Schmuziger M. Normothermic Versus Hypothermic Perfusion During Primary Coronary Artery Bypass Grafting. Cardiovascular Surgery $1995 ; 3: 519-524$.

107. Cook DJ. Changing Temperature Management for Cardiopulmonary Bypass. Anesthesia \& Analgesia 1999; 88: $1254-1271$. 
108. Insler SR, O'Connor MS, Leventhal MJ, Nelson DR, and Starr NJ. Association Between Postoperative Hypothermia and Adverse Outcome After Coronary Artery Bypass Surgery. Annals of Thoracic Surgery 2000; 70: $175-181$.

109. Grigore AM, Grocott HP, Mathew JP, Phillips-Bute B, Stanley TO, Butler A, Landolfo KP, Reves JG, Blumenthal JA, Newman MF, and the Neurologic Outcome Research Group of the Duke Heart Center. The Rewarming Rate and Increased Peak Temperature Alter Neurocognitive Outcome After Cardiac Surgery. Anesthesia \& Analgesia 2002; 94:4 - 10.

110. Shaaban $M$, Harmer $M$, and Kirkham F. Cardiopulmonary Bypass Temperature and Brain Function. Anaesthesia 2005;60:365-372.

111. Sellke FW, DiMaio JM, Caplan LR, Ferguson TB, Gardner TJ, Hiratzka LF, Isselbacher EM, Lytle BW, Mack MJ, Murkin JM, and Robbins RC. Comparing On-Pump and Off-Pump Coronary Artery Bypass Grafting. Numerous Studies but Few Conclusions. A Scientific Statement From the American Heart Association Council on Cardiavascular Surgery and Anesthesia in Collaboration With the Interdisciplinary Working Group on Quality of Care and Outcomes Research. Circulation 2005; 111:2858-2864.

112. Heres EK, Marquez J, Malkowski MJ, Magovern JA, and Gravlee GP. Minimally Invasive Direct Coronary Artery Bypass: Anesthetic, Monitoring, and Pain Control Considerations. Journal of Cardiothoracic and Vascular Anesthesin 1998; 12:385 - 389.

113. Abramov D, Tamariz MG, Fremes SE, Guru V, Borger MA, Christakis GT, Bhatnagar G, Sever JY, and Goldman BS. Trends in Coronary Artery Bypass Surgery Results: A Recent 9-Year Study. Annals of Thoracic Surgery 2000; 70:84-90.

114. Ferguson Jr TB, Hammill BG, Peterson ED, DeLong ER, and Grover FL for the STS National Database Committee. A Decade of Change - Risk Profiles and Outcomes for Isolated Coronary artery Bypass Grafting Procedures, 1990-1999: A Report From the STS National Database Committee and the Duke Clinical Research Institute. Annals of Thoracic Surgery 2002;73:480 -490 .

115. Wilmore DW and Kehlet H. Recent Advances. Management of Patients in Fast Track Surgery. British Medical Journal 2001; 322: $473-476$.

116. Kehlet $\mathrm{H}$ and Dahl JB. Anaesthesia, Surgery, and Challenges in Postoperative Recovery. The Lancet 2003;362: $1921-1928$.

117. Atom KV, Emery RW, Petersen RJ, and Schwartz M. Cost-Effectiveness and Predictors of Early Extubation. Annals of Thoracic Surgery 1995; 60:127-132.

118. Cheng DCH. Early Extubation After Cardiac Surgery Decreases Intensive Care Unit Stay and Costs. Pro: Early Extubation After Cardiac Surgery Decreases Intensive Care Unit Stay and Costs. Journal of Cardiothoracic and Vascular Anesthesia 1995; 9: 460 - 464.

119. Lee JIH, Kim KH, vanHeeckeren DW, Murrell HK, Cmolik BL, Graber R, Effron B and Geha AS. Cost Analysis of Early Extubation After Coronary Bypass Surgery. Surgery 1996;120:611 - 619.

120. Cheng DCH. Impact of Early Tracheal Extubation on Hospital Discharge. Journal of Cardiothoracic and Vascular Anesthesia 1998; 12, Suppl 2: 35- 40.

121. Wong DT, Cheng DCH, Kustra R, Tibshirani R, Karski J, Carrol-Munro J, and Sandler A. Risk factors of Delayed Extubation, Prolonged Length of Stay in the Intensive Care Unit, and Mortality in Patients Undergoing Coronary Artery Bypass Graft with Fast-track Cardiac Anesthesia. Anesthesiology 1999; 91: 936 - 944.

122. Nashef SAM, Roques F, Michel P, Gauducheau E, Lemeshow S, Salamon R, the EuroSCORE Study Group. European System for Cardiac Operative Risk Evaluation (EuroSCORE). European Journal of Cardio-Thoracic Surgery $1999 ; 16: 9-13$.

123. Geissler HJ, Holzl P, Marohl S, Kuhn-Regnier F, Mehhorn U, Sudkamp M, and de Vive R. R isk Stratification in Heart Surgery: Comparison of Six Score Systems. European Journal of CardioThoracic Surgery 2000; 17: 400 - 406 . 
124. Michel P, Roques F, Nashef SAM, The EuroSCORE Project Group. Logistic or Additive EuroSCORE for High-Risk Patients? European Journal of Cardio-Thoracic Surgery 2003; 23: $684-687$.

125. Michalopoulos A, Tzelepis G, Pavlides G, Kriaras J, Dafni U, and Geroulanos S. Determinants of Duration of ICU Stay After Coronary Artery Bypass Graft Surgery. British Journal of Anaesthesia 1996; 77:208-212.

126. Ott RA, Gutfinger DE, Miller MP, Selvan A, Codini MA, Alimadadian H, and Tanner TM. Coronary Artery Bypass Grafting "On Pump": Role of Three-Day Discharge. Annals of Thoracic Surgery 1997; 64: $478-481$.

127. Capdeville M, Lee JH, and TaylorAL. Effect of Gender on Fast-Track Recovery After Coronary Artery Bypass Graft Surgery. Journal of Cardiothoracic and Vascular Anesthesia 2001; 15:146 $-151$.

128. Millar K, Asbury AJ, and Murray GD. Pre-Existing Cognitive Impairment as a Factor Influencing Outcome After Cardiac Surgery. Britsh Journal of Anaesthesia 2001; 86: 63 - 76

129. Walthall $H$, Robson D, and Ray S. Do Any Preoperative Variables Affect Extubation Time after Coronary Artery Bypass Graft Surgery? Heart \& Lung 2001; 30: 216 - 224.

130. Parr KG, Patel MA, Dekker R, Levin R, Glynn R, Avorn J, and Morse DS. Multivariated Predictors of Blood Product Use in Cardiac Surgery. Journal of Cardiothoracic and Vascular Anesthesia 2003; 17: $176-181$.

131. Wigfield CH, Lindsey JD, Munoz A, Chopra P'S, Edwards NM, and Love ER. Is Extreme Obesity a Risk Factor for Cardiac Surgery? An Analysis of Patients with a BME $\geq 40$. European Journal of Cardio-Thoracic Surgery 2006; 29: $434-440$.

132. London MJ, Shroyer AL, Coll JR, MaWhinney S, Fullerton DA, Hammerrmeister KE, and Grover FL. Early Extubation Following Cardiac Surgery in a Veterans Population. Anesthesiology 1998; 88: 1447- 1458.

133. Welsby IJ, Bennet-Guerrero E, Atwell D, White WD, Newman MF, Smith PK, and Mythen MG. The Association of Complication Type with Mortality and Prolonged Stay After Caridiac Surgery with Cardiopulmonary Bypass. Anesthesia \& Analgesia 2002; 94:1072 - 1078.

134. Reich DL, Bodian CA, Krol M, Kuroda M, Osinski T, and Thys DM. Intraoperative Hemodynamic Predictors of Mortality, Stroke, and Myocardial Infarction After Coronary Artery Bypass Surgery. Anesthesia \& Analgesia 1999; 89:814-822.

135. Ridderstolpe L, Ahlgren E, Gill H, and Rutberg H. Risk Factor Analysis of Early and Delayed Cerebral Complications After Cardiac Surgery. Journal of Cardiothoracic and Vascular Anesthesia $2002 ; 16: 278-285$.

136. Stanley TO, Mackensen GB, Grocott HP, White WD, Blumenthal JA, Laskowitz DT, Landolfo KP, Reves JG, Mathew JP, Newman MF, The Neurologic Outcome Research Group, and The CARE Investigators of the Duke Heart Center. The Impact of Postoperative Atrial Fibrillation on Neurocognitive Outcome After Coronary Artery Bypass Graft Surgery. Anesthesia \& Analgesia $2002 ; 94: 290-295$

137. Athanasiou T, Aziz O, Mangoush O, Al-Ruzzeh S, Nair S, Malinovski V, Casula R, and Glenville B. Does Off-Pump Coronary Artery Bypass Reduce the Incidence of Post-Operative Atrial Fibrillation? A Question Revisited. European Journal of Cardio-Thoracic Surgery 2004; 26:701 -710 .

138. McMurry SA and Hogue Jr CV. Atrial Fibrillation and Cardiac Surgery. Current Opinion in Anaesthesiology 2004; 17:63 - 70.

139. Sackett DL, Straus SE, Richardson WS, Rosenberg W, and Haynes RB. Evidence-Based Medicine. How to Practice and Teach EBM. Churchill Livingstone, second edition, 2000, London.

140 Jamrozik K. Why Evidence-Based Medicine? Best Practice \& Research Clinical Anaesthesiology $2001 ; 15: 505-518$.

141. Pedersen T. Evidence-Based Anaesthesia and Health Economics. Best Practice \& Research Clinical Anaesthesia 2006; 20:347 - 360 . 


\section{CHAPTER 3}

Aims and outline of the thesis 
In developing a clinical pathway protocol for fast-track cardiac surgery in low-risk coronary artery surgery patients in the University Hospital in Maastricht, intense communication, education, cooperation, and teamwork was necessary among all parties involved in this clinical pathway. These parties included anaesthesiologists, cardiac surgeons, cardiologists, intensivists, administrators, nurses in the $\mathrm{OR}$ and in the ICU and many others.

After studying the literature and after evaluating our own clinical experience, we still encountered several problems for which we had no answers. To answer these questions, we formulated several research questions and initiated several clinical trials in order to develop a clinical pathway protocol for these patients that was safe, cost-effective and evidence-based:

\section{Chapter 4: How to select patients eligible for fast-tracking?}

To answer this question, we reviewed the literature for risk scoring and risk evaluating scoring systems that would serve our patients best.

\section{Chapter 5: Which anaesthetic drug is best in fast-tracking?}

We investigated the potential of the new ultra-short acting opioid remifentanil in fast-tracking and compared the effects of remifentanil with another short-acting opioid on operative haemodynamic stability, on the suppression of noxious stimuli, and on postoperative pain and extubation times.

Chapter 6: Can anaesthetics decrease the SIRS to cardiac surgery?

We compared the effects of several anaesthetic techniques on the postoperative systematic inflammatory response syndrome. Attenuating this response may decrease morbidity and makes the postoperative course more uneventful.

\section{Chapter 7: Does off-pump surgery decreases postoperative morbidity?}

We compared a group of coronary artery surgery patients undergoing surgery with cardiopulmonary bypass with an off-pump group. The hypothesis was that avoiding cardiopulmonary bypass would result in less inflammation with less organ damage.

Chapter 8: Is less and cheaper monitoring safe in fast-tracking?

We investigated whether the new dynamic parameter Stroke Volume Variation (SVV) better predicts the cardiac responsiveness to fluid therapy after cardiac surgery as compared to the old static parameters central venous pressure (CVP) and pulmonary capillary wedge pressure (PCWP), using the less invasive technique of pulse contour analysis.

Chapter 9: Is the implementation of our clinical pathway protocol safe and cost-effective? In a randomized clinical trial including 600 patients, we investigated whether our clinical pathway protocol including early postoperative extubation, rapid discharge within 8 hours from the ICU to a step-down unit and early hospital discharge, was safe and cost-effective. 


\section{CHAPTER 4}

\section{Risk stratification for adverse outcome in cardiac surgery}

J. H. Heijmans ${ }^{1,3}$, J. G. Maessen ${ }^{2,3}$, P. M. H.J. Roekaerts ${ }^{1,3}$

University Hospital Maastricht, Departments of ${ }^{1}$ Anesthesiology and ${ }^{2}$ Cardiothoracic Surgery; ${ }^{3}$ Cardiovascular Research Institute of Maastricht (CARIM), Maastricht, The Netherlands

European Journal of Anaesthesiology 2003; 20: 515-527. 


\section{SUMMARY}

Risk-adjusted outcome prediction is mainly important in two separate fields. The first is quality monitoring: measuring actual versus predicted mortality in an institution allows assessment of the clinical surgical and anaesthesia performance while adjusting for the risk profile of the patients. Without risk stratification, surgeons and hospitals treating high-risk patients will appear to have worse results than others. This may prejudice referral patterns, affect the allocation of resources and even discourage the treatment of high-risk patients. The second field is that of informed consent and clinical decision-making. Risk-adjusted predicted mortality should form an important part of patient and surgeon decisions on whether or not to proceed with surgery. Clearly, no 'perfect' model can be produced as some aspects of mortality will always be related to risk factors not included in the model (e.g. the quality of the distal coronary artery vessels in coronary artery surgery) or due to chance happenings not related to preoperative patient characteristics (such as surgical error). An individual patient will either survive or die after cardiac surgery. Clearly, no scoring system will predict the specific outcome for every patient. However, risk stratification will inform patients and clinicians of the likely risk of death for a group of patients with a similar risk profile undergoing the proposed operation. This information is useful and should form part of the basis on which the patient and surgeon decide whether to proceed. 


\section{INTRODUCTION}

Improvements in medical treatment and recent trends in interventional cardiology have altered the profile of patients referred for cardiac surgery. The proportion of high-risk patients has increased dramatically with greater numbers of elderly patients, those requiring re-operation and patients with a greater number of associated illnesses presenting for cardiac surgery.

A major problem in contemporary cardiac surgery and anaesthesia is quantification of operative risk. An accurate and objective estimate of perioperative risk would have important consequences for patients, clinicians and administrators. Such estimates could be used to help weigh the risks of surgical and conservative treatment; to advise individual patients of their perioperative risk; to allow fair comparison of outcome and costs between surgical units and within units between surgeons; to facilitate quality assurance by allowing comparison of outcomes from year to year, or before and after a change in practice; and, controversially, to aid selection of patient treatment based on risk versus predicted cost.

Risk-adjusted outcome prediction is thus mainly important in two separate fields. The first is quality monitoring: measuring actual versus predicted mortality in an institution allows assessment of clinical surgical and anaesthesia performance while adjusting for the risk profile of the patients. Regardless of the weaknesses of the scoring systems, such monitoring is important so that major underperformance does not go undetected for long, and so that measures can be taken to correct underperformance before the risk to patients is significantly increased. Listed by region and by hospital, such data are also useful in clinical research to assess the prevalence of cardiovascular disease, the effectiveness of therapy and trends in medical practice. The second field is that of informed consent and clinical decisionmaking. Risk-adjusted predicted mortality should form an important part of patient and surgeon decisions on whether or not to proceed with surgery.

Outcome data have also become important to payers of healthcare and insurance carriers, since the cost of surgery is highly influenced by the frequency of complications as well as the duration of intensive care unit (ICU) and total postoperative stay. Changes in healthcare financing have resulted in an emphasis on systematizing clinical practices in the care of cardiac surgical patients. As a means to reduce costs, the development and institution of clinical pathways have become commonplace. At the same time, the rapid advancement of invasive cardiology techniques has led to a dramatic increase in the preoperative risk profile of cardiac surgical patients. To build and maintain cardiac surgery programmes, clinicians are faced not only with the challenge of maximizing clinical efficiency, but also with that of critically assessing individual patient risk in relation to cost and finding ways to manage high-risk patients more effectively.

In a world of limited medical resources but increasing demand for medical services, rationing is something with which all members of the medical profession 
and their patients are familiar. Much time and effort have been spent trying to identify patients who would benefit from cardiac surgery and attempting to prioritize their position on waiting lists. However, it was quickly recognized that decisions about priority and urgency were often inconsistent. Therefore, a simple and objective scoring system for establishing surgical priority is warranted. In addition, clinicians are often required to identify patients who are likely to require a short period of high-dependency care following cardiac surgery. The number of available ICU beds may be limited by a large number of long-stay patients or, more commonly, because of a shortage of trained cardiac ICU nurses. When resources in the ICU are sparse, it is necessary to identify a group of patients with a high probability of requiring less than $24 \mathrm{~h}$ ICU care in order that some operations can be carried out each day. Prospective planning of resource utilization (ICU staffing and bed allocation) is therefore necessary.

Risk stratification systems such as the Goldman index have not been validated well when applied to cardiac surgical patients, and the Acute Physiologic and Chronic Health Evaluation (APACHE) scoring system not only requires the use of postoperative data, but also involves assessment of variables that are deliberately manipulated (temperature, blood pressure, heart rate, arterial $\mathrm{pH}$, level of consciousness) at the time of ICU arrival. Application of the APACHE system to the cardiac surgical population results in overprediction of mortality in patients with high scores, probably because of artificially high initial scores associated with many rapidly reversible physiological abnormalities often seen immediately after heart surgery. Cardiac surgery remains a very complex area for outcome prediction. Outcome prediction models can be broadly classified as disease-specific or general. The general severity of illness systems for critically ill patients such as APACHE I, II, III, Simplified Acute Physiology Score (SAPS I, II), and Mortality Probability Models (MPM I, II) have matured over the last two to three decades. Among the specific outcome prediction models, in 1989 Parsonnet and colleagues elaborated a method of uniform risk stratification for evaluation of the results of surgery in acquired adult heart disease.' Since that time, several other cardiac surgery risk models have been developed and attempts made to predict mortality and morbidity following cardiac surgery.

In this paper, the most important systems for cardiac operative risk evaluation will be reviewed. Criteria by which to evaluate risk-adjusted outcomes programmes in cardiac surgery will be discussed. As quality monitoring is now one of the requirements of good medical practice, this review will stress the need for using an operative risk scoring system as this is a tool by which quality monitoring can be achieved. 


\section{Criteria by which to evaluate risk-adjusted outcomes models}

Narrowly defined, the purpose of risk adjustment is to account for patient characteristics that influence patient outcomes. Broadly defined, risk adjustment seeks to account for all factors other than processes and structures of care that may explain variation in patient outcomes. Three factors may account for observed differences in outcomes: differences in significant risk factors among patients, random variation, or difference in the processes or structures of care. Focusing on outcomes adjusted for risk is an indirect way of studying the differences in processes or structures of care. Random variation is taken into account through the application of a variety of statistical techniques. Evaluating an adequate risk-adjustment system to assess the contribution of patient risk factors requires consideration of several criteria that are outlined below.

\section{Outcomes of interest}

In-hospital or hospitalization-associated mortality is the outcome that has been most frequently studied. In the field of cardiac surgery, mortality and survival are clearly important outcomes. The bypass grafting procedure carries with it a small, but significant, risk of death and, for some subsets of the population of patients with atherosclerotic cardiovascular disease and angina refractory to medical therapy, the procedure is performed to prevent death. Complications and potentially preventable morbidity after the grafting procedure are also important outcomes. Imprecise definitions of these events and the human tendency to under report these events in medical records may limit the reliable identification of these non-fatal outcomes. For patients and their families, outcomes after discharge, such as the ability of patients to function independently, to interact with families and friends and to return to work and daily routines, are critically important. Unfortunately, as the interval after the procedure lengthens, the ability to collect information about the patients' functional status and quality of life becomes more difficult. The science of measuring these outcomes has made considerable strides in the past 20 years, but measurement instruments that are both widely used and accepted by the clinical community are still only emerging. Consequently, hospital-associated mortality is the most widely used outcome, partly because it is easy to measure and readily available. Ideally, a range of pertinent outcomes (i.e. mortality, morbidity, changes in functional status and quality of life, cost of care, and patient-reported perceptions of the non-technical aspects of care) should be reported for each procedure.

\section{Time frame}

The time frame or window of observation should be determined for each outcome. Some options include the duration of hospitalization, a fixed period (e.g. 30 days) after the procedure, the episode of care (e.g. from the onset of symptoms to 
the time of cardiac rehabilitation), or a variable period initiated by the patient's admission to the hospital, by the performance of a procedure, or by the diagnosis of a condition. The window of observation may vary according to each outcome. To eliminate bias, it is better to identify a fixed period in terms of days or weeks after the procedure instead of the in-hospital period for determining post-procedural morbidity and mortality. The time frame for each outcome also influences the nature of significant patient risk factors. For example, the patient risk factors for 30 day mortality may be significantly different from the risk factors for functional status at 6 months postoperatively. Acute clinical stability is the most important component of risk for studies involving short time frames. Chronic disability, physical functioning and various non-clinical factors increase in importance as the time window expands.

\section{Units of observation}

Inlerently a comparative exercise, the most appropriate comparisons in outcomes assessment depend on who will be using the risk-adjusted outcome information and their purposes. Depending on the context, the unit of observation may be the individual patient or it may be groupings of patients according to individual surgeons, groups of surgeons, a hospital or groups of hospitals. The units are not mutually exclusive in the sense that smaller units are nested within larger units. In cardiac surgery, individual surgeons or groups of surgeons as well as hospitals are compared. The use of the individual patient as a unit of observation deserves special attention. Some investigators advocate the use of these risk models as clinical prediction rules. ${ }^{2}$ Diamond argues that, for example, the predicted probability of death obtained by entering an individual patient's risk factors into the risk model generates information that may be helpful to that patient and to his/her physician in making decisions about whether to pursue cardiac surgical treatment. Although this predicted probability of death might be one of many helpful pieces of clinical information available to the patient and surgeon, caution should be used in interpreting this predicted probability of death. First, the predicted probability of death generated by these risk-adjustment models reflects the likelihood of death for that patient if he/ she were operated on in the average hospital by the average surgeon of all the hospitals and surgeons in the database from which the models were developed, and not the predicted probability with respect to the surgeon whom the patient has consulted. Second, the uncertainty about the predicted probability of death for an individual patient is usually great. These predicted probabilities of death should be shared with patients cautiously and with the stated understanding that the accuracy of the prediction is quite limited. 


\section{Data sources}

Three sources of data are available for risk adjustment: discharge abstract information, retrospective chart review and prospective primary data furnished by the medical care team or others in the hospital. Primary data collection is more costly but more reliable.

\section{Data collecting}

The patient care team is the most appropriate group to collect the data because they are most familiar with by the medical care team or others in the hospital. Primary data collection is with the patient. Some resistance to collecting this information may exist among care providers because the use of risk-adjusted outcome information may be perceived as either irrelevant to the care of the patient or as part of a punitive exercise in quality assurance. Some clinicians may report that patients have risk factors that they do not have in order to inflate the risk profile of their patients and thus improve their risk-adjusted outcomes. Random audits of institutions can control a potential systematic bias in these systems.

\section{Standardization of definitions}

Training, standard reference manuals of terms and definitions, and readily available resources for assistance in the form of summary sheets, help screens in computer software or networks of data collectors who can answer questions all help to standardize the collection of risk factors and outcome data. Newsletters and regular meetings or conference calls may help to communicate changes in data collection systems, and frequent updates or new variables and definitions help to improve the reliability of data collection.

\section{Data entering}

Paper-and-pencil forms may be used and the data on them forwarded later to a central database, or a personal computer on the clinical care floors may be used and the data later transferred by electronic means to the central database.

\section{Reliability of data}

The reliability of the data needs to be assessed. The most important aspect of these reliability assessments is to establish reliability between data collectors. These reliability tests determine whether two trained data collectors find the same values of risk and outcome variables in the risk models on independent reviews of the patient and his/her care. 


\section{Sample population}

Predictive risk-adjusted models for coronary artery bypass grafting have been developed in voluntary regional or state alliances, state-mandated systems, national voluntary systems, and nationally mandated comprehensive systems. Most of the systems strive to collect data on every coronary artery bypass graft procedure performed within each hospital.

\section{Sampling period}

Given that it takes several years to build these databases because of the large number of cases needed to create stable models, the data in the database should be recent enough to represent both the current population of patients undergoing cardiac operations and the current practice of cardiac surgery.

\section{Database size}

The appropriate size of the database used to develop risk-adjustment models depends on the incidence of the outcome of interest, the number of patient risk factors to be included in the model and the statistical modelling approach. In studies of operative mortality after cardiac operations, stepwise logistic regression analysis as well as other methods should be performed, provided the number of deaths is at least 10 times greater than the number of predictor variables. As a rule of thumb, more than 2000 cases are necessary to develop stable logistic regression models for comparing hospitals or cardiac surgeons for a procedure such as coronary artery bypass grafting with a baseline mortality rate of 3-5\% and a reasonable number (i.e. $7-15$ ) of potential predictive variables.

\section{Statistics}

Multiple analytical issues must be considered in developing risk-adjustment models: data reduction, the structure of continuous variables such as age, clinical and statistical criteria for the selection of important risk factors for each outcome to be studied, and the choice of the most appropriate multivariate modelling techniques. Detailed information on these statistical issues is described elsewhere. ${ }^{3}$ For a good understanding of this review, we give a short description of the 'Bayes' theorem' and of the 'receiver operating characteristic curve'. Bayes set out his theory of probability in his Essay Towards Solving a Problem in the Doctrine of Chances, which was published in the Philosophical Transactions of the Royal Society of London (1764). Bayes' theorem is the formula that provides the relation between the prior odds and post odds:

$$
\text { Post odds = likelihood ratio } x \text { prior odds, }
$$




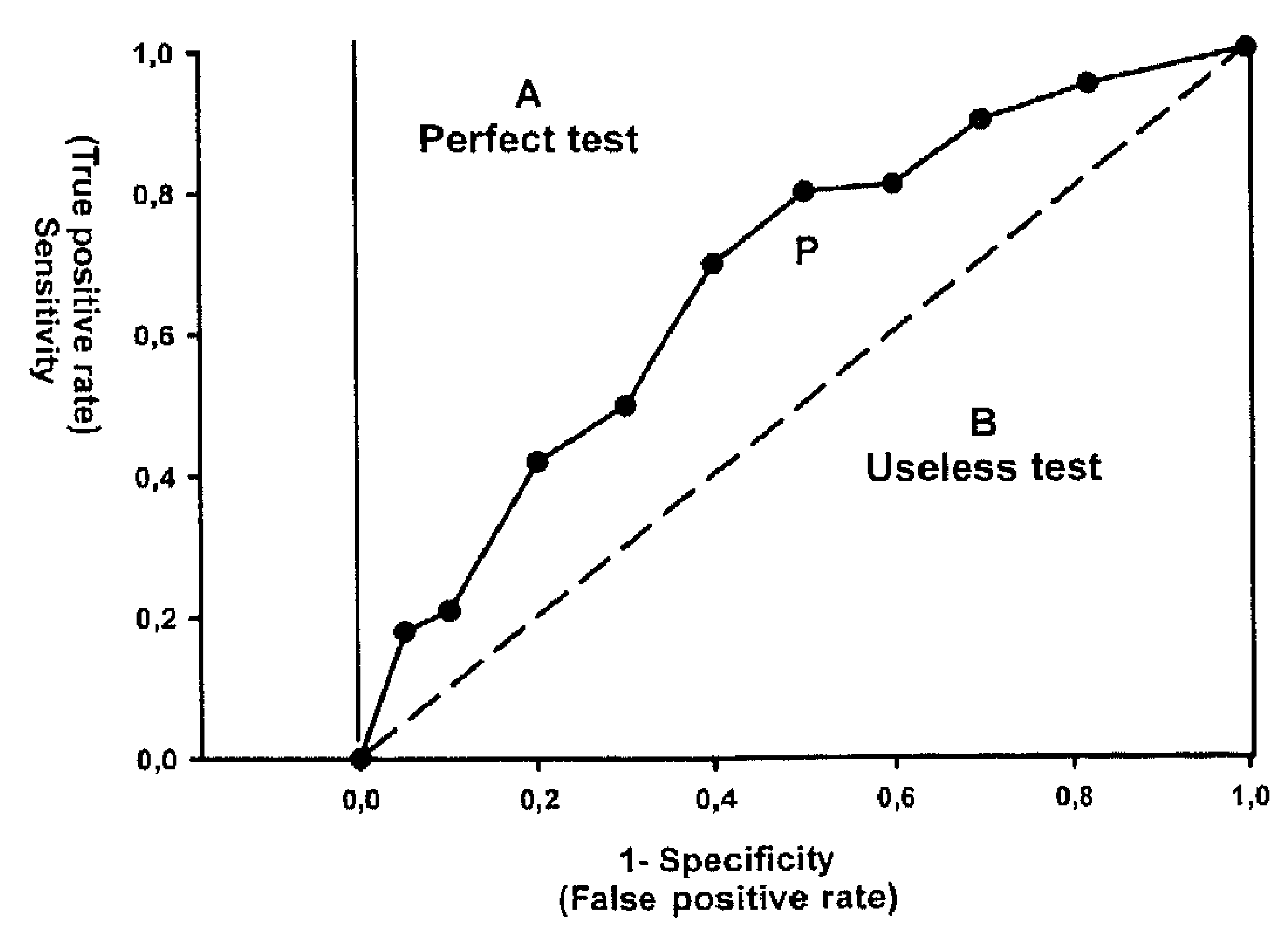

Figure 1. Receiver operating characteristic ROC curves.

The curve is a means of evaluating predictive tests that give a continuous output rather than a positive or negative value; it avoids the need to choose an arbitrary cut-offlevel. The curve is obtained by graphing sensitivity of the test for predicting an outcome (the true-positive rate) against 1-specificity (the false-positive rate), with each possible value of the test as a cut-off. The area under the curve is proportional to the probability of correctly predicting an individual outcome. Three ROC curves are shown. (A) Ideal test, without false-positives or -negatives, with a curve that runs from the origin to a point with a sensitivity of 1 and false-positive rate of 0 . (B) Curve for a test that produces results by chance. (P): Parsonnet score.

where the likelihood ratio expresses how much more likely it is that a patient with a given condition should fall among those who die rather than survive. The area under the receiver operating characteristic (ROC) curve is believed to be an appropriate statistical measure of the ability of a model to predict what it intends. It is a plot of sensitivity versus 1 - specificity, and the area under the curve is a useful summary measure of the diagnostic accuracy of the tool. An area of 1 suggests a perfect predictor, and a value of 0.5 is a test of no value. Areas of between 0.5 and around 0.7 represent a rather low accuracy - the true-positive proportion is not much greater than the false-positive proportion. Values between 0.7 and 0.9 represent a high accuracy (Fig. 1).

\section{Validity of the model}

Validity in risk adjustment systems is a multidimensional concept. Some dimensions of validity have to do with the accuracy of the data and the precision of the measures, and others have to do with the justifiability of the inferences drawn from the data. In assessing the validity of risk-adjustment methods, the most important types of validity are face validity, content validity, criterion or construct validity, attributional validity, and predictive validity. A detailed discussion of these issues may be found elsewhere. ${ }^{4}$ 
Table 1. Components of the Multivariate Logistic Regression Model

\begin{tabular}{|c|c|c|}
\hline Risk Factor & $\begin{array}{r}\text { Logistic } \\
\text { Coefficient B } \\
\end{array}$ & $\mathrm{p}$ \\
\hline Age & 0.054 & $<0.001$ \\
\hline Aortic valve disease & 0.235 & NS* \\
\hline Bypass only & -0.588 & 0.038 \\
\hline Bypass plus another procedure & 0.647 & 0.002 \\
\hline Elevated cholesterol & 0.083 & NS \\
\hline Diabetes & 0.456 & $<0.001$ \\
\hline Catastrophic states & 1.455 & $<0.001$ \\
\hline Family history & -0.065 & NS \\
\hline Female gender & 0.509 & $<0.001$ \\
\hline Hypertension & 0.263 & 0.028 \\
\hline Left ventricular aneurysm & -0.553 & NS* \\
\hline Left ventricular ejection fraction & 0.271 & 0.001 \\
\hline Mitral valve disease & 0.835 & $<0.001$ \\
\hline Obesity & -0.271 & $0.027^{\star}$ \\
\hline Preoperative intra-aortic balloon pump & 1.473 & $<0.001$ \\
\hline Reoperation & 0.893 & $<0.001$ \\
\hline Smoking & 0.089 & NS \\
\hline
\end{tabular}

NS, not significant; Regression intercept $B_{11}=-7.032, p<0.001 ;$ * Factors were predictive of increased risk of operative mortality in univariate analysis.

\section{Missing data}

Generally, the more complete the data set, the more valid the prediction model. Therefore, it is axiomatic that the first recording of the data should be as complete as possible. However, given that the problem of missing data will occur, there are two general approaches to dealing with this situation. The first is to impute the missing data, which means inserting a value for the missing data item. Imputation techniques may simply use the mean of the known values for that variable or estimate the missing value on the basis of the other characteristics of the patient using multivariate regression. The second general approach to dealing with missing data is to use a statistical model that does not drop the case if the value of one of the variables is missing. These techniques include classification and regression trees and the Bayesian model.

\section{Refinement of the model}

Given the changes in the risk profile of patients undergoing cardiac operations and the changes in the process of care, risk models need to be refined periodically. The optimal timing for recalibrating the models is not known. Significant changes in patient risk profiles or practice innovations should stimulate re-examination and potential revision of the models. ${ }^{5}$ 
Table 2. Components of the additive model.

\begin{tabular}{|c|c|}
\hline Risk factor & Assigned weight \\
\hline Female gender & 1 \\
\hline Morbid obesity & 3 \\
\hline Diabetes (unspecified type) & 3 \\
\hline Hypertension (systolic BP $>140 \mathrm{mmHg}$ ) & 3 \\
\hline \multicolumn{2}{|l|}{ Ejection fraction (\%): (actual value when available) } \\
\hline Good $( \pm 50)$ & 0 \\
\hline Fair $(30-49)$ & 2 \\
\hline Poor $(<30)$ & 4 \\
\hline \multicolumn{2}{|l|}{ Age $(y r)$} \\
\hline $70-74$ & 7 \\
\hline $75-79$ & 12 \\
\hline$\geq 80$ & 20 \\
\hline \multicolumn{2}{|l|}{ Reoperation } \\
\hline First & 5 \\
\hline Second & 10 \\
\hline Preoperative IABP & 2 \\
\hline Left ventricular aneurysm & 5 \\
\hline Emergency surgery following PTCA or & 10 \\
\hline \multicolumn{2}{|l|}{ catheterization complications } \\
\hline Dialysis dependency (PD or Hemo) & 10 \\
\hline $\begin{array}{l}\text { Catastrophic states (e.g., acute structural defect, } \\
\text { cardiogenic shock, acute renal failure) }{ }^{\star}\end{array}$ & $10-50^{+}$ \\
\hline $\begin{array}{l}\text { Other rare circumstances (e.g., paraplegia, pacemaker dependency, } \\
\text { congenital HD in adult, severe asthma) }\end{array}$ & $2-10^{+}$ \\
\hline \multicolumn{2}{|l|}{ Valve surgery } \\
\hline Mitral & 5 \\
\hline PA pressure $\pm 60 \mathrm{mmHg}$ & 8 \\
\hline Aortic & 5 \\
\hline Pressure gradient $>120 \mathrm{mmHg}$ & 7 \\
\hline$C A B G$ at the time of valve surgery & 2 \\
\hline
\end{tabular}

BP, blood pressure; IABP, intra-aortic balloon pump; PTCA, percutaneous transluminal coronary angioplasty; PD, peritoneal dialysis; Hemo: hemodialysis; $\mathrm{HD}$, heart disease; $\mathrm{PA}$, pulmonary artery; $\mathrm{CABG}$, coronary artery bypass graft: $\star$ On the actual worksheet, these risk factors require justification: + Values were predictive of increased risk of operative mortality in univariate analysis.

\section{Risk-stratification models}

\section{Parsonnet model}

Although several risk-stratification methods had been devised in the past, it was only in 1989 that Victor Parsonnet (Medical Director and Pacemaker Center Director of Surgical Research, Beth Israel Medical Center, Newark, NJ, USA) described a straightforward, uniform reporting system - applicable to all adult open-heart surgical procedures and based upon objective risk factors - that left 
Table 3. French score

\begin{tabular}{lc}
\hline Risk factor & Weighting index \\
\hline 1. Age $70-74$ & 3 \\
Age $75-79$ & 4 \\
Age $\geq 80$ & 5 \\
2. Chronic renal failure: creatinine $\geq 200 \mu \mathrm{mol} \mathrm{L}^{-1}$ & 5 \\
Chronic renal failure on dialysis & 6 \\
3. Ejection fraction $30-50 \%$ & 2 \\
Ejection fraction $\leq 30 \%$ & 5 \\
4. Saphenous vein graft only & 2 \\
5. Reoperation & 2 \\
6. Tricuspid surgery & 4 \\
7. Valve + CABG & 2 \\
8. Critical situations: & 15 \\
Myocardial infarct $<48 \mathrm{~h}$ & 4 \\
Ventricular tachycardia/fibrillation & 4 \\
Preoperative intubation & 4 \\
Transplantation & 4 \\
Post MI VSD & 9 \\
Acute aortic dissection & 4 \\
Pulmonary embolectomy & 4 \\
\hline
\end{tabular}

little room for observer bias. Uni- and multivariate logisticregression analyses were performed on data pertaining to 3500 consecutive open-heart surgery procedures selected retrospectively from an existing database covering a 5 yr period between 1982 and 1987. Relatively objective risk factors were assessed individually for significance with $\chi 2$-test analysis for categorical data, and $t$-test and one-way analysis of variance for continuous data. The criteria for inclusion of a variable were the following:

- Predictive value must be demonstrated by univariate analysis.

- Data must be available for every patient.

- Data must be available at every institution.

- Variable must be as free as possible from subjectivity or bias.

- Variable must be simple and direct (not derived from other information).

Beginning with an imaginary 'ideal' patient (e.g. age $<70 \mathrm{yr}$, left ventricular ejection fraction $=50 \%$, aortocoronary bypass only, first operation), retrospective analyses were performed with the risk factors listed in Table 1 taken one at a time. 
Table 4. EuroSCORE

Definition Score

Patient-related factors

Age

Sex

Chronic pulmonary disease

Extracardiac arteriopathy

Neurologic dysfunction

Previous cardiac surgery

Serum creatinine

Active endocarditis

Critical preoperative state

Cardiac-related factors

Unstable angina

LV dysfunction

Recent myocardial infarction

Pulmonary hypertension

Operation-related factors

Emergency

Other than isolated $\mathrm{CABG}$

Surgery on thoracic aorta

Postinfarct septal rupture
Per 5 years or part there of over 60 years 1

Female

Longterm use of bronchodilatators or steroids for lung disease 1

Any one or more of the following: claudicatio, carotid occlusion 2 or $>50 \%$ stenosis, previous or planned intervention on the abdominal aorta, limb arteries or carotids

Diseases severely affecting ambulation or day-to-day functioning 2

Requiring opening of the pericardium 3

$>200 \mu \mathrm{mol} \mathrm{L}-1$ preoperatively 2

Patient still under antibiotic treatment for endocarditis at the 3 time of surgery

Any one or more of the following: ventricular tachycardia or 3 fibrillation or aborted sudden death, preoperative cardiac massage, preoperative ventilation before arrival in the anaesthetic room, preoperative inotropic support, intraaortic balloon counterpulsation or preoperative acute renal failure (anuria or oliguria $<10 \mathrm{ml} \mathrm{h}^{-1}$ )

Rest angina requiring i.v. nitrates until arrival in the anaesthetic room

Moderate or LVEF $30-50 \%$

Poor or LVEF $<30$

$<90$ days 2

Systolic PA pressure $>60 \mathrm{mmHg}$

Carried out on referral before the beginning of the next working 2 day than or in addition to CABG

Major cardiac procedure other

2

For disorder of ascending, arch or descending aorta 3

Operative mortality was defined as any death occurring within 30 days of surgery. Odds ratio tests were used to determine the odds of dying with each risk factor. The significance level was chosen as $\mathrm{P}<0.05$. Those risk factors found to be significant were then assigned a value equal of that to that of the odds ratio, and an additive model was constructed in the form:

$$
\mathrm{P}=\mathrm{k}_{1}+\mathrm{k}_{2}+\ldots+\mathrm{k}_{15} \text {, }
$$

where $\mathrm{P}$ is the predicted probability of operative mortality and $\mathrm{k}_{1}-\mathrm{k}_{15}$ are the nonzero risk scores assigned for each applicable risk factor listed in Table 2. Seventeen factors that met the above criteria were analysed separately for association with operative mortality. A second model was constructed in the form: 


$$
P=\left(1+1 e^{z}\right)^{-1}
$$

where $\mathrm{P}$ is the predicted probability of death within 30 days, $\mathrm{Z}=\mathrm{B}_{0}+\mathrm{B}_{1} \mathrm{X}_{1}+\ldots+$ $B_{17} X_{17}$, and $B_{1}-B_{17}$ are the logistic coefficients shown in Table 2 . With two exceptions, each of the risk factors $X_{1}-X_{17}$ was entered as 1 (present) or 0 (absent). The exceptions were age $\left(\mathrm{X}_{1}=\right.$ age $\left.(\mathrm{yr})\right)$ and left ventricular ejection fraction $\left(\mathrm{X}_{12}=1\right.$ for ejection fraction $=50 \%, 2$ for ejection fraction between 30 and $49 \%, 3$ for ejection fraction $<30 \%$ ). A few other remarks need to be made about the components of the additive model (Table 2). Concerning gender, it is the consensus that females have a higher operative mortality than males, perhaps because of smaller artery size. Whatever the cause, females tend to fare less well than males. Diabetes mellitus was considered present if there was a history of diabetes, whether or not antidiabetic agents had been taken and regardless of the duration of disease. Hypertension was included if the blood pressure exceeded $140 / 90 \mathrm{mmHg}$ or if the patient had a history of high blood pressure or was taking antihypertensive medication. Ventricular aneurysm was included only if the aneurysm was resected. Otherwise, its presence was considered to have been reflected in the ejection fraction. Concerning catastrophic states and other rare circumstances, an estimate of risk was made by the surgical resident. Some risk factors were excluded as too subjective, or were available only after an operation, or were indefinable or were not universally available. These include chronic obstructive pulmonary disease, the number of cardiopulmonary bypass $(\mathrm{CPB})$ operations, the use of the internal mammary artery, the presence and degree of main stem stenosis, left ventricular end-diastolic pressure, extracranial cerebrovascular disease, CPB time, operative priority, and the severity of the atherosclerotic lesions. The two models were compared by regression analysis to test the validity of the additive univariate model. Three hundred earlier cases from the same database were then tested retrospectively by summation of all the risk factor scores applicable to each case; the total score was then compared with the actual outcome. The weights given to each risk factor were tested by regression analysis of those 300 cases. The correlation coefficient was 0.99 , which was regarded as adequate justification for a subsequent prospective study of 1322 cases with the additive model above. The predicted probability of operative mortality for the univariate model was stratified into ranges reflecting patients' expected mortality rate: good risk (0-4\%), fair risk (5-9\%), poor risk (10-14\%), high risk $(15-19 \%)$ and extremely high risk $(=20 \%)$. The probabilities of operative mortality for each patient, as determined with the two models, were compared, using linearregression analysis, to test the validity of the univariate additive model. The method was also used to analyse the relationship of risk to postoperative complications and to length of hospital stay. The system was tested prospectively in four other institutions. Of 17 variables (Table 1) subjected to univariate analysis, 15 fulfilled the purposes of the study (Table 2). The mean predicted operative mortality rate was 
$10.4 \%$. The mean observed operative mortality rate was $8.9 \%$. Comparing between predicted and actual outcomes for each of the five risk ranges revealed a correlation coefficient of 0.99 by regression analysis. A comparison of the uni- and multivariate models showed a correlation coefficient of 0.85 . To test the assumption that any kind of heart surgery might fit the predictive score, three general types of operations were compared: aortocoronary bypass alone, valves alone and valves plus aortocoronary bypass. The operative mortality in each subgroup resembled the results of the groups combined. The differences were not statistically significant. A comparison of the overall results obtained at the primary institution with those from the two other centres testing this method and the results observed at the three centres taken as a unit, with the mortality rates predicted by the additive model, showed no statistically significant difference. Further analysis showed that the duration of hospitalization and the incidence of non-fatal complications (including atrial fibrillation, hypokalaemiarelated dysrhythmias, stitch infections) was distinctly related to the severity of the disease.

\section{Other North American risk-stratification models}

The multivariate estimation of risk of in-hospital or perioperative mortality has been studied by several investigators the past three decades. ${ }^{(-1)}$

After the publication of the Parsonnet score in 1989, several other risk-stratification models were developed for predicting morbidity and mortality at cardiac surgery, including the scores described by Hannan and colleagues in 1990, ${ }^{10}$ O'Connor and colleagues in 1992, ${ }^{11}$ Higgins and colleagues in 1992, ${ }^{12}$ Tuman and colleagues in 1992, ${ }^{13}$ Tremblay and colleagues in 1993, ${ }^{14}$ Edwards and colleagues in $1994^{15}$ and $\mathrm{Tu}$ and colleagues in $1995^{16}$ The Society of Thoracic Surgeons National Cardiac Surgery Database model developed by Edwards and colleagues is the most widely used model in the USA and is an unparalleled effort in terms of its size and comprehensiveness. The current Bayesian equation being used incorporates 23 risk factors: age, female gender, left ventricular function, emergency/ urgent surgery, repeat operation, renal dysfunction, diabetes mellitus, hypertension, left ventricular aneurysm, morbid obesity, catastrophic states, previous myocardial infarction, left main disease, unstable angina, cerebrovascular disease, chronic obstructive pulmonary disease, cardiogenic shock, current tobacco use, intravenous nitrates, intravenous inotropic support, more than 100 pack-year smoking, percutaneous transluminal coronary angioplasty emergency, valvular disease, one-, two-, and three-vessel disease. The disadvantages of the Society of Thoracic Surgeons database are that it is commercially protected, participation is expensive and outcome prediction requires a computer.

$\mathrm{Tu}$ and colleagues developed a simple six-variable risk index (age, gender, left ventricular function, type of surgery, urgency of surgery, repeat operation). 
Mortality, ICU length of stay and postoperative length of stay after cardiac surgery could be predicted using this risk index.

\section{$U K$}

There are differences between the European and North American populations for coronary artery bypass grafting and the North American algorithms appear not to be very useful for predicting mortality in the UK. The UK national score was developed using Bayes' theorem. Bridgewater and colleagues compared four predictive models (Society of 'Thoracic Surgeons risk program, Ontario Province Risk Score, Parsonnet score, UK national scores); these programs all had areas under the ROC curves ranging from 0.60 to $0.75^{17}$

The Parsonnet score and preliminary version of the UK national scores seem to be the best tools available at present. Even these only have limited ability to predict observed outcome, as shown by the area under the ROC curve of 0.73 and 0.75 , respectively, and great care should still be exercised when using these models. Wynne-Jones and colleagues also highlighted the limitations of the Parsonnet score for measuring risk-stratified mortality in the North West of England in a prospective study on 8210 patients undergoing cardiac surgery between April 1997 and March 1999. The area under the ROC curve in this study was 0.74 is

\section{France}

In France, the area under the ROC curve using Parsonnet's score was 0.6519 Therefore, this score is not an appropriate choice as a standard assessment method in France. Seven risk factors in the Parsonnet system were not predictive according to multivariate analysis: gender, hypertension, left ventricular aneurysm, intra-aortic balloon pump, morbid obesity, mitral and aortic valve surgery. A 'French score' was therefore developed ( 42 centres, 7181 patients). Clear, simple and well-defined variables were selected.

Four variables were deliberately excluded in spite of their good predictive value: unstable angina, peripheral vascular disease, chronic obstructive pulmonary disease and congestive heart failure. Significant variations from one centre to another led to the consideration that these risk factors could not be clearly defined and therefore lacked objectivity. Eight simple and objective risk factors were selected (Table 3). Point weighting was assigned to each risk factor according to the statistical value. A simple cumulative score was thereby established to classify patients within four incremental risk groups:

- Group A: score $<2$

- Group B: 2-3

- Group C: 4-6

- Group D: $>6$ 
In the study by Roques and colleagues, the French score was predictive for mortality (area under ROC curve $=0.75$ ) and for severe morbidity (0.74). Because of the importance of epidemiological factors, the direct application of US systems in Europe is questionable. Socio-economic conditions, living standards, healthcare funding, and geographic and ethnic origins should be taken into account when using a scoring system. A wide consensus about a scoring system is recommended. As socio-economic and epidemiological conditions are supposed to be (or to become) uniform enough within the European Union, a selfassessment system based on a large database reflecting current practices in cardiac surgery in Europe is now being developed: the EuroSCORE (Table 4)

\section{EuroSCORE}

To construct a scoring system for the prediction of early mortality in cardiac surgical patients in Europe on the basis of objective risk factors, the Euro- SCORE database was developed. ${ }^{20}$ It was divided into developmental and validation subsets. In the former, risk factors deemed to be objective, credible, obtainable and difficult to falsify were weighed on the basis of regression analysis. An additive score of predicted mortality was constructed. Its calibration and discrimination characteristics were assessed in the validation dataset. Thresholds were defined to distinguish low- (EuroSCORE 1-2), moderate- (3-5) and high-risk (>6) groups. The developmental dataset had 13302 patients, calibration by Hosmer Lemeshow $x^{2}$ (goodness-of-fit testing) was $(8)=8.26(\mathrm{P}<0.40)$ and discrimination by area under ROC curve was 0.79 . The validation dataset had 1479 patients, calibration $\mathrm{x}^{2}(10)$ $=7.5(\mathrm{P}<0.68)$ and the area under the ROC curve was 0.76 .

\section{Comparison of risk-stratification models}

Weightman and colleagues compared in 1997 the Parsonnet, Higgins, Tremblay and Tu scores. ${ }^{21}$ Any of the scores could be used to estimate perioperative nisk and compare outcome between coronary surgery units, but none has sufficient specificity and sensitivity to identify individuals who will experience an adverse outcome. Therefore, none of the risk scores can be considered adequate screening tests for perioperative mortality. It is well known by epidemiologists that effective screening in a population with a low incidence of adverse events requires a test with high specificity. Although the risk scores are not sufficiently accurate to predict individual outcomes, the four scores had areas of $0.65-0.75$ under their ROC curves, indicating moderate usefulness as predictive tests. Therefore, the scores may be useful in comparisons of outcomes between groups of patients. Because all four are equally good at stratifying risk, it is immaterial which is used, as long as all cardiac surgery units agree. The Parsonnet score is a candidate for a universally applicable score. As already mentioned, it was introduced in 1989 with the specific intention of comparing preoperative risk and outcomes between units. Advantages 
are that it can be applied to all cardiac surgery, not just coronary artery surgery, and that is already in widespread use. However, it has the disadvantages of moderate complexity and significant observer discretion in point allocation. In contrast, the Tu score can be simply calculated from a limited set of data readily available for most patients undergoing heart surgery. Another proposed method of uniform data collection, described above, is the Society of Thoracic Surgeons database. In 1997, Pliam and colleagues published a comparative analysis of coronary surgery riskstratification models (Parsonnet, Higgins and Society of Thoracic Surgeons). ${ }^{22}$ These models could accurately discriminate outcome about $80 \%$ of the time. While such accuracy might be useful in aggregate decisionmaking, it would not appear to be justifiable to deny a treatment procedure to a given patient solely on the basis of a high-risk profile generated by any of these models. In contrast to the limited degree of accuracy for individual patients, each of the models under investigation provided reasonable assessments of overall population risk within their respective $95 \%$ confidence interval (mean \pm 1.98 standard deviations). When applied to relatively large numbers of patients, these models can be useful in managing risk, which relies upon the law of large numbers. Similarly, Pinna- Pintor and colleagues in 2002 described the inaccuracy of four coronary surgery risk-adjusted models to predict mortality in individual patients (Parsonnet, Higgins, French score and EuroSCORE). ${ }^{23}$ The four presurgical predictive models were similarly able to discriminate favourable versus unfavourable outcomes and highly accurate to predict overall mortality.

Lawrence and colleagues in 2000 described the Parsonnet score as a good predictor of postoperative complications and of the duration of ICU stay following cardiac surgery. ${ }^{24}$ Data in their study were prospectively collected on 5591 patients in London, UK. This finding is of value in selecting a cohort of patients likely to maintain a smooth flow of patients through the cardiothoracic unit when resources are limited to a few free ICU beds. In 2000, Doering and colleagues showed that the Parsonnet score, an established mortality risk measure, was a useful indicator of both ICU and hospital costs. ${ }^{25}$ Risk-adjusted mortality has been used to compare institutions as a whole or surgeons. Nashef and colleagues in 2001 showed that the relationship between Parsonnet's predicted mortality and the actual mortality at Papworth Hospital (Cambridge, UK) varied between surgeons. ${ }^{26}$ Individual surgeons may have strengths and weaknesses, which are related to preoperative risk stratification. Pinna-Pintor and colleagues showed, using the Parsonnet score, that institutions can affect mortality of patients undergoing open heart operations, regardless of the surgeons involved. ${ }^{27}$ The investigators compared the outcome of a surgical team working in two different hospitals. Based on a relatively small series of patients, Zenati and colleagues suggested that risk models geared for standard coronary bypass grafting were not appropriate for minimally invasive coronary bypass. ${ }^{28}$

We are far from having a gold standard; the proliferation of presurgical predictive methods is an indication of the inadequacy of the available models. The first 
version of Parsonnet's method is based on a surgical population operated on about 15 years ago. The mortality observed in that database is higher than the one usually obtained at present. The problem of the transferability of predictive models to other countries, other institutions or other times must be taken into consideration.

\section{Determinants of ICU length of stay after coronary artery bypass graft surgery}

Patients who have prolonged ICU stays may be exposed to increased risk of infection, delayed mobilization and prolonged isolation from the support of family and social contacts. Therefore, early identification of patients at risk for prolonged ICU stay will aid clinicians in planning prophylactic measures to prevent complications associated with longer ICU stays. Several investigators have identified preoperative factors associated with prolonged ICU stays in coronary artery bypass graft patients, including recent myocardial infarction, smoking, number of diseased arteries and preoperative left-ventricular end-diastolic pressure. Studies of postoperative factors associated with prolonged ICU stays have indicated that low cardiac output syndrome, postoperative use of inotropes, atrial dysrhythmias, respiratory complications and renal insufficiency contribute to prolonged ICU stays. Only two studies have included intraoperative variables, such as aortic cross-clamp time and CPB time, that were correlated with ICU duration when considered singularly. ${ }^{29,30}$ Because timely identification of patients likely to require prolonged ICU stays offers the opportunity to plan efficient use of resources, studies have considered specific models. The APACHE II score has been suggested as a predictive measure, but it is limited by variations in measurement modalities and requires extensive data collection.

Over the past decade, several studies have developed predictive indices of stay in the ICU after cardiac surgery. These studies used group comparisons or examined predictors of long versus short ICU stays. The majority of these studies has included preoperative variables and, in general, have not taken into account events that affect patient outcome during the operative or immediate postoperative period. A great disparity in the type and number of independent variables has been reported. Tuman and colleagues found 11 variables to be significantly associated with stay in the ICU, including emergency surgery, age, preoperative renal dysfunction, previous myocardial infarction, cerebrovascular disease, type of surgery, congestive heart failure and left ventricular dysfunction. Although the duration of stay in the ICU in this study correlated with a clinical risk score derived from these variables, no accurate predictive index of stay in the ICU based on clinical score was provided. Furthermore, it cannot be determined which risk factor is the most significant predictor of length of stay in the ICU. The Tuman model has not been well tested.

The Parsonnet score, an established mortality risk measurement system highly predictive of 30 day operative mortality and closely related to overall complication 
rate and duration of postoperative hospitalization, has not been evaluated as an indicator of ICU length of stay.

In a similar study of preoperative variables, Tu and colleagues suggested that length of stay in the ICU after cardiac surgery could be predicted by a multivariate predictive index. Five variables were found to be statistically significant independent predictors for a prolonged stay in the ICU (age, female gender, left ventricular function, urgency of surgery, type of surgery). A predictive index was developed for length of stay in the ICU with a risk score of $0-12$. Patients with a low-risk score remained in the ICU for a few days, while patients with a high-risk score had a prolonged stay in the ICU. However, this method has a poor predictive ability; $31.7 \%$ of patients with a lowrisk score (0-3) stayed $>2$ days in the ICU, whereas only $69.4 \%$ of patients with a high-risk score $(>8)$ had a prolonged $(>2$ days) stay in the ICU. The authors acknowledged that length of stay in the ICU could never be predicted with certainty.

Tu and colleagues reported 1 year later that duration of stay in the ICU after cardiac surgery could be predicted using a six-variable risk index, which included age, gender, preoperative ejection fraction, type of surgery, urgency of surgery and reoperation. Although the authors considered prolonged stay in the ICU as $>6$ days, the overall predictive ability of their index was poor (67\%).

Weintraub and colleagues showed that the correlates of prolonged hospital stay could not be predicted by preoperative variables alone.$^{30}$ The authors emphasized that serious complications were responsible for prolonging the length of stay after coronary artery bypass grafting surgery.

A fundamental different study is that by Michalopoulos and colleagues, ${ }^{31}$ because they took into account not only preoperative variables, but also significant variables related to both the operative and immediate postoperative period, such as the number of inotropes administered and blood transfusions performed. They found that the duration of stay in the ICU depended predominantly on a single critical variable, the number of inotropes administered during the immediate postoperative period. This is not surprising because inotropic support reflects poor cardiac function. It follows that the group requiring intensive inotropic support would include patients with intraoperative complications (e.g. myocardial ischaemia or infarction), inadequate revascularization, low cardiac output syndrome related to systemic inflammatory response syndrome (SIRS), stunned myocardium or inadequate myocardial protection during bypass. Apparently, none of these conditions can be predicted accurately by any preoperative variable. On the other hand, in some high-risk cases (e.g. low ejection fraction) there may be great improvement in left ventricular function after operation because of successful revascularization. This is supported by Zaroff and colleagues who found that although low preoperative ejection fraction is a known predictor of poor immediate postoperative outcome after cardiac surgery, not all patients with low preoperative ejection fraction required inotropic support. ${ }^{32}$ In both cases, the models based 
on preoperative variables are likely to fail in predicting stay in the ICU. Doering and colleagues also addressed the issue of determinants of ICU length of stay after coronary artery bypass graft surgery. ${ }^{33}$ This is the first study evaluating ICU length of stay in a multivariate linear regression model with pre-, intra- and postoperative variables. This study also evaluated the usefulness of the Parsonnet score as a prognostic indicator of ICU length of stay. Bivariate correlations of ICU length of stay with four preoperative, three intraoperative and nine postoperative variables were conducted to identify potential criterion variables for multiple linear regression. One preoperative variable (Parsonnet score) and four postoperative variables (length of intubation, presence of dysrhythmias, early haemodynamic instability, 12 $\mathrm{h}$ fluid balance) explained $45.6 \%$ of the variance in ICU length of stay. Intraoperative variables were not independent correlates of ICU length of stay. Classification as 'extremely high' risk by Parsonnet scoring (score 20) yielded a positive predictive value of $84 \%$ for ICU stay $>1$ day. Negative predictive value was $42.8 \%$. Thus, pre- and postoperative variables explained a large portion of the variance in ICU stay after coronary artery bypass grafting. Although the Parsonnet score was not helpful in identifying patients who require only a short ICU stay, it may help clinicians screen for patients likely to require stays $>1$ day and plan appropriate use of resources in the ICU. The ideal indicator would accurately identify patients requiring prolonged ICU stay as well as those likely to need only short stays. The Parsonnet score has a fairly high positive predictive value, indicating that classification in the extremely high category correctly identifies patients requiring ICU stays $>1$ day $84 \%$ of the time. However, the negative predictive value of the 'extremely high' Parsonnet score is low. This means that patients who require ICU stays of 1 day are classified incorrectly almost half of the time. The difficulty in identifying accurate prognostic indicators of ICU length of stay probably results from the wide variability among ICU patients in general and the large number of variables that could be considered. Although the Parsonnet score may be useful in identifying patients who need prolonged ICU care, it is not useful in identifying patients who need only short ICU stays.

\section{DISCUSSION}

The development of an appropriate risk-stratification model is an important goal in cardiac surgery, and an ideal tool should be easy to implement, be objective, be an accurate predictor of observed mortality and be in widespread use, allowing comparison between surgeons and units to be made readily. No existing tool fulfils all of these criteria. The use of risk-stratified mortality studies for analysing surgical results is obviously a developing area. There is a need for further validation studies on a larger scale than has been performed to clarify potential errors in databases and to see how such errors can influence the results of this type of analysis. It may be that changing techniques of data collection, modifying definitions or omitting 
various subjective risk factors from the predictive models will be necessary to obtain robust conclusions and eliminate the potential for gaming. Clearly, no "perfect' model can be produced as some aspects of mortality will always be related to risk factors not included in the model (e.g. the quality of the distal vessels) or due to chance happenings not related to preoperative patient characteristics (such as surgical error). Understanding the ability of the predictive model is important, but of greater importance is the way the models can be used to define the linits of acceptable practice and be incorporated into programmes to improve standards.

Those who provide, purchase and use healthcare recognize that the resources for such care are limited. It is now established that the cost of treatment must be taken into consideration in decisions about healthcare provision. Future debate in this field will focus on the quality of treatment and the measurements of this quality. In cardiac surgery, it has long been accepted that operative or hospital mortality is an indicator of quality of care. This is true to a large extent: death following heart surgery is often due to failure to achieve a satisfactory cardiac outcome, itself the cause of major early morbidity as well as poor longterm results. Crude operative mortality fails as a measure of quality only when there are major variations in case mix. For operative mortality to remain a valid measure of quality of care, it must be related to the risk profile of the patients receiving surgery, hence the need for a reliable risk-stratification model already recognized by earlier workers in this field. There is another reason for the development and regular use of risk stratification in the assessment of cardiac surgical results. Doctors and hospitals operate in an increasingly open system where the availability of results and public accountability may influence decisionmaking. Without risk stratification, surgeons and hospitals treating high-risk patients will appear to have worse results than others. This may prejudice referral patterns, affect the allocation of resources and even discourage the treatment of high-risk patients. This is especially undesirable in cardiac surgery because it is precisely this group of patients that stands to gain most from surgical treatment, in spite of the increased risk. Risk stratification helps eliminate the bias against high-risk patients. An individual patient will either survive or die after cardiac surgery. Clearly, no scoring system will predict the specific outcome for every patient. However, risk stratification will inform patients and clinicians about the likely risk of death for a group of patients with a similar risk profile undergoing the proposed operation. This information is useful and should form part of the basis on which the patient and surgeon decide whether to proceed. The Parsonnet score has been criticized for the nature of its statistical derivation in that it systematically overestimates mortality, particularly for high-risk patients, and because its scoring system is quite subjective, again especially in the high-risk group. It also omits many surgeons" favourite' risk factors such as the number of coronary vessels diseased, the urgency of operation and the presence of chronic obstructive pulmonary disease. It also contains several risk factors not associated with mortality. The score has also been customized in different ways by different centres. Nevertheless, it has strengths 
in that it is accepted by the world-wide cardiac surgical community, it is easy to use without computer assistance and it has been shown to have the ability to predict observed mortality in adult cardiac surgery within the useful range. The Society of Thoracic Surgeons National Cardiac Surgery Database model is the most widely used model in the USA and is an unparalleled effort in terms of its size and comprehensiveness. The disadvantages of that database are that it is commercially protected, participation is expensive and the outcome prediction requires a computer.

The EuroSCORE database is large, up to date, and unrivalled in completeness and accuracy. It is also derived from a cross-section of contemporary European cardiac surgery. It is therefore an appropriate database for the construction of a risk evaluation scoring system for Europe. The transition from database to scoring system sacrifices some precision for the sake of simplicity. A compromise must be reached so that the system recognizes common risk factors, can provide some degree of risk prediction yet remains simple enough to use at the point of delivery of care. The existence of the scoring system does not preclude full use of the database, when resources permit, for more precise analysis. The true test of the EuroSCORE is in its widespread application in the field. Doctors should put it to the test in their hospitals, overall and in individual patient and procedural subgroups, in relation to operative mortality, to major morbidity and to the use of resources. Quality monitoring is now one of the requirements of good medical practice. 


\section{REFERENCES}

1. Parsonnet $\mathrm{V}$, Dean $\mathrm{D}$, Bernstein $\mathrm{AD}$. A method of uniform stratification of risk for evaluating the results of surgery in acquired adult heart disease. Circulation 1989: 79; I3-I12.

2. Diamond GA. Future imperfect: the limitation of clinical prediction models and the limits of clinical prediction. J Am Coll Cardiol 1989; 14: 12-22.

3. Glantz SA. Primer of Biostatistics. New York, USA: McGraw Hill Book Company, 2001.

4. Donabedian A. The definition of quality and approaches to its assessment. Explorations in quality assessment and monitoring. Vol 1. Ann Arbor, MI, USA: Health Administration Press, 1980.

5. Daley J. Criteria by which to evaluate risk-adjusted outcomes programs in cardiac surgery. Ann Thorac Surg 1994; 58: 1827-1835.

6. Loop FD, Berrettoni JN, Pichard A, Siegel W, Razavi M, Effer DB. Selection of the candidate for myocardial revascularization; a profile of high risk based on multivariate analysis. $J$ Thorac Cardiovasc Surg 1975;69:40-51.

7. Kennedy JW, Kaiser GC, Fisher LD, et al. Multivariate discriminant analysis of the clinical and angiographic predictors of operative mortality from the Collaborative Study in Coronary Artery Surgery (CASS).J Thorac Cardiovasc Surg 1980; 80: 876-887.

8. Wright JG, Pifarre R, Sullivan $\mathrm{HJ}$, et al. Multivariate discriminant analysis of risk factors for operative mortality following isolated coronary artery bypass graft. Loyola University Medical Center experience, 1970 to 1984 . Chest 1987; 91:394-399.

9. Edwards FH, Albus RA, Zajtchuk R, et al. Use of a Bayesian statistical model for risk assessment in coronary artery surgery. Ann Thorac Surg 1988; 45: 437-440.

10. Hannan EL, Kilburn H Jr, O'Donnell JF, Lukacik G, Shields EP. Adult open heart surgery in New York State. An analysis of risk factors and hospital mortality rates.JAMA 1990;264:2768-2774.

11. O'Connor GT, Plume SK, Olmstead EM, et al.Multivariate prediction of in-hospital mortality associated with coronary artery bypass graft surgery. Northern New England Cardiovascular Disease Study Group. Circulation 1992; 85:2110-2118.

12. Higgins TL, Estafanous FG, Loop FD, Beck GJ, Blum JM, Paranandi L. Stratification of morbidity and mortality outcome by preoperative risk factors in coronary artery bypass patients. A clinical sevenity score. JAMA 1992; 267: 2344-2348.

13. Tuman KJ, McCarthy RJ, March RJ, Najafi H, Ivankovich AD. Morbidity and duration of ICU stay after cardiac surgery. A model for preoperative risk assessment. Chest 1992; 102: 36-44.

14. Tremblay NA, Hardy JF, Permalt J, Carrier M. A simple classification of the risk in cardiac surgery: the first decade. Can J Anaesth 1993; 40:103-111.

15. Edwards FH, Clark RE, Schwartz M. Coronary artery bypass grafting: the Society of Thoracic Surgeons National Database experience. Ann Thorac Surg 1994; 57: 12-19.

16. Tu JV, Jaglal SB, Naylor CD. Multicenter validation of a risk index for mortality, intensive care unit stay, and overall hospital length of stay after cardiac surgery. Steering Committee of the Provincial Adult Cardiac Care Network of Ontario. Circulation 1995;91:677-684.

17. Bridgewater $B$, Neve $H$, Moat $N$, Hooper T, Jones M. Predicting operative risk for coronary artery surgery in the United Kingdom: a comparison of various risk prediction algorithmus. Heart 1998; 79:350-355.

18. Wynne-Jones K, Jackson M, Grotte G, Bridgewater B. Limitations of the Parsonnet score for measuring risk stratified mortality in the north west of England. The North West Regional Cardiac Surgery Audit Steering Group. Heart 2000; 84:71-78.

19. Roques F, Gabrielle F, Michel P, De Vincentiis C, David M, Baudet E. Quality of care in adult heart surgery: proposal for a self-assessment approach based on a French multicenter study. Eur J Cardiothorac Surg 1995; 9: 433-439.

20. Nashef SA, Roques F, Michel P, Gauducheau E, Lemeshow S, Salamon R. European system for cardiac operative risk evaluation (EuroSCORE). Eur J Cardiothorac Surg 1999; 16: 9-13. 
21. Weightman WM, Gibbs NM, Sheminant MR, Thackray NM, Newman MA. Risk prediction in coronary artery surgery: a comparison of four risk scores. Med J Aust 1997; 166: 408-411.

22. Pliam MB, Shaw RE, Zapolanski A. Comparative analysis of coronary surgery risk stratification models. J Invas Cardiol 1997; 9:203-222.

23. Pinna-Pintor $\mathrm{P}$, Bobbio $\mathrm{M}$, Colangelo $\mathrm{S}$, et al. Inaccuracy of four coronary surgery risk-adjusted models to predict mortality in individual patients. Eur J Cardiothorac Surg 2002; 2: 199-204.

24. Lawrence DR, Valencia O, Smith EE, Murday A, Treasure T. Parsonnet score is a good predictor of the duration of intensive care unit stay following cardiac surgery. Heart 2000; 83: 429-432.

25. Doering LV, Esmailian F, Laks H. Perioperative predictors of ICU and hospital costs in coronary artery bypass graft surgery. Chest 2000;118:736-743.

26. Nashef SA, Carey F, Charman S. The relationship between predicted and actual cardiac surgical mortality: impact of risk grouping and individual surgeons. Eur J Cardiothorac Surg 2001; 19 : $817-820$.

27. Pinna Pintor P, Bobbio M, Sandrelli L, et al. Risk stratification for open heart operations: comparison of centers regardless of the influence of the surgical team. Ann Thorac Surg 1997; 64: $410-413$

28. Zenati M, Cohen HA, Holubkov $\mathrm{R}$, et al. Preoperative risk models for minimally invasive coronary bypass: a preliminary study. J Thorac Cardiovasc Surg 1998; 116: 584-589.

29. Christakis GT, Ivanov J, Weisel RD, Birnbaum PL, David TE, Salerno TA. The changing pattern of coronary artery bypass surgery. Circulation 1989;80: I151-I161.

30. Weintraub WS, Jones EL, Craver J, Guyton R, Cohen C. Determinants of prolonged length of hospital stay after coronary bypass surgery. Circulation 1989; 80:276-284.

31. Michalopoulos A, Tzelepis G, Pavlides G, Kriaras J, Dafni U, Geroulanos S. Determinants of duration of ICU stay after coronary artery bypass graft surgery. Br J Anaesth 1996; 77:208-212.

32. Zaroff J, Aronson S, Lee BK, Feinstein SB, Walker R, Wiencek JG. The relationship between immediate outcome after cardiac surgery, homogeneous cardioplegia delivery, and ejection fraction. Chest 1994; 106: 38-45.

33. Doering LV, Esmailian F; Imperial-Perez F, Monsein S. Determinants of intensive care unit length of stay after coronary artery bypass graft surgery. Heart Lung 2001; 30: 9-17. 


\section{CHAPTER 5}

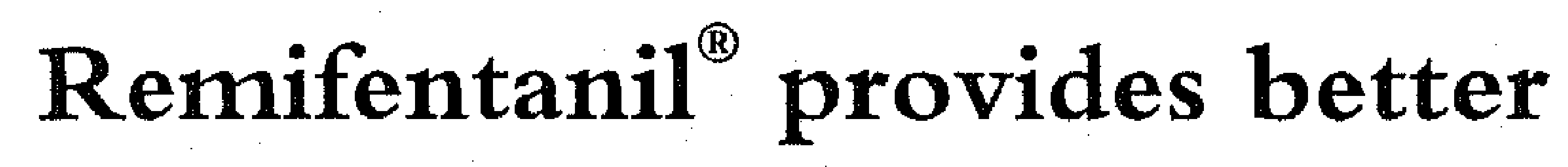
protection against noxious stimuli during cardiac surgery than Alfentanil ${ }^{\circledR}$

J. H. Heijmans ${ }^{1}$, J. G. Maessen ${ }^{2}$, P. M. H. J. Roekaerts ${ }^{1}$

University Hospital Maastricht, Departments of ${ }^{1}$ Anaesthesiology and ${ }^{2}$ CardioThoracic Surgery, Maastricht, The Netherlands

European Journal of Anaesthesiology 2004; 21: 612-618. 


\section{SUMMARY}

Background and objective: We hypothesized that remifentanil-propofol cardiac anaesthesia, plus administration of pirinitranide (piritramide) upon cessation of the remifentanil infusion, would be associated with a shorter time to tracheal extubation than alfentanil-propofol anaesthesia, without the occurrence of major haemodynamic instability.

Methods: Haemodynamic stability and recovery characteristics of two remifentanil infusion regimens $\left(0.5 \mu \mathrm{g} \cdot \mathrm{kg}^{-1} \cdot \mathrm{min}^{-1} ; 0.25 \mu \mathrm{g} \cdot \mathrm{kg}^{-1} \cdot \mathrm{min}^{-1}\right)$ were therefore compared with an alfentanil infusion regimen $\left(1 \mu \mathrm{g} \cdot \mathrm{kg}^{-1} \cdot \mathrm{min}^{-1}\right)$, in combination with target-controlled infusion of propofol, in a randomized blinded trial in 75 coronary artery surgery patients.

Results: Pirinitramide provided good postoperative analgesia without prolonging extubation times: median extubation time in minutes after stopping the opioid-sedative drugs was 300 in the higher-dose remifentanil group and 270 in the lower-dose remifentanil group and alfentanil group $(P=0.606)$. Significant timeby-treatment interactions were seen for systolic arterial pressure $(P=0.015)$, mean arterial pressure $(P=0.009)$ and diastolic arterial pressure $(P=0.006)$. No significant interaction $(P=0.489)$ and no constant treatment effect were seen for heart rate $(P=0.288)$. Time effects were highly significant $(P<0.0001)$ for all haemodynamic variables. Heart rate remained stable in all groups. In the higherdose remifentanil group, blood pressure was significantly different and lower during surgery and in this group less bolus doses of the opioid-sedative drugs $(P=0.015)$ had to be given.

Conclusion: The higher-dose remifentanil infusion provided superior suppression of haemodynamic responses to noxious stimuli with better haemodynamic stability. 


\section{INTRODUCTION}

The development of total intravenous (i.v.) anaesthesia and the widespread acceptance of propofol in cardiac anaesthesia have led to an increased interest in the combination of propofol with fentanyl, sufentanil or alfentanil..$^{1-3}$ The pharmacokinetic-based drug infusion systems, target-controlled infusion (TCI), can rapidly achieve and maintain a constant blood concentration of i.v. anaesthetic drugs $^{4.5}$ allowing more precise drug titration and, as a result, fast recovery." Alfentanil has been considered a very suitable opioid to use in combination with propofol, because of its pharmacokinetics, rapid onset of action and short duration of action. However, its context-sensitive halftime increases with the duration of infusion and reaches a plateau after approximately $1 \mathrm{~h}^{7}$ Due to its potency and predictable pharmacokinetic characteristics, remifentanil, an ultra short-acting opioid agonist with a close concentration-effect relationship, may be the ideal opioid to use in combination with propofol for continuous i.v. administration.8 Its brief and constant context-sensitive half-time, however, mandates a specific strategy for postoperative pain relief." The administration of a morphine derivative just before stopping the remifentanil infusion may be a simple and effective strategy for achieving good postoperative pain control. ${ }^{10-12}$ However, this technique could jeopardize early extubation after a remifentanil-based anaesthetic technique.

There are several reports about severe cardiovascular depression with remifentanil in patients undergoing cardiac surgery; ${ }^{13.14}$ these cardiovascular effects could be, in part, attributed to the dose and method of administration of remifentanil.

We hypothesized that remifentanil-TCI propofol anaesthesia with the administration of the synthetic morphine derivative pirinitramide (piritramide) upon cessation of the remifentanil administration would produce a shorter time to tracheal extubation compared with an alfentanil-TCI propofol anaesthetic technique. To test this hypothesis, we compared two infusion regimens of remifentanil with an earlier described alfentanil infusion regimen ${ }^{3}$ and included detailed haemodynamic assessments. Two remifentanil concentrations were studied since the optimal dose of remifentanil providing adequate anaesthesia without cardiovascular instability, has not yet been described.

\section{METHODS}

The study was approved by our hospital's Ethics Committee and written informed consent was obtained from 75 patients who were randomized into a higher dose remifentanil group (HDRG), a low-dose remifentanil group (LDRG) or an alfentanil group (AG), in combination with a propofol-TCI technique. The study was blinded for the opioid infusion. Exclusion criteria included left ventricular ejection fraction of less than $25 \%$, hypothermic circulatory arrest, recent 
myocardial infarction, preoperative inotropic or intraaortic balloon pump support, emergency surgery and significant pulmonary, endocrine, metabolic, or neurological diseases. All patients were receiving chronic $B$-adrenoceptor blocking drugs. The distribution of other medications affecting cardiovascular variables (calcium antagonists, ACE inhibitors, nitrates) was the same in all groups. All these other medications were stopped the evening before surgery. Patients were premedicated with lorazepam, administered together with their individual $\beta$-adrenoceptor blocking drug. Upon arrival in the operation room, patients received $7.5 \mathrm{~mL} . \mathrm{kg}^{-1}$ of a glucose-salt containing infusion and baseline haemodynamic data $\left(T_{1}\right)$ were obtained: heart rate (HR), systolic, mean and diastolic arterial pressure (SAP, MAP, DAP). During the induction of anaesthesia, this solution was administered to maintain cardiac filling pressures at their normal values (central venous pressure (CVP) between 6 and $12 \mathrm{mmHg}$ ). After preoxygenation and baseline haemodynamic measurements, a precurarization dose of pancuronium $20 \mu \mathrm{g} \cdot \mathrm{kg}^{-1}$ was administered to the patient. Immediately after this, both the alfentanil (Rapifen ${ }^{(B)}$, Janssen Pharmaceutica, Beerse, Belgium) or remifentanil (Ultiva ${ }^{(B)}$, GlaxoSmithKline, Zeist, The Netherlands) and propofol infusions were administered with the BD Master Diprifusor $^{\text {(i) }}$ TCI system (software developed by the University of Glasgow, UK).

Three groups of patients were compared: Group 1 (HDRG): a loading dose of remifentanil $2.5 \mu \mathrm{g} \cdot \mathrm{kg}^{-1}$ was infused over $4 \mathrm{~min}$; thereafter, remifentanil was infused at a maintenance rate of $0.5 \mu \mathrm{g} \cdot \mathrm{kg}^{-1} \cdot \mathrm{min}^{-1}$ throughout surgery. Group 2 (LDRG): a loading dose of remifentanil $2.5 \mu \mathrm{g} . \mathrm{kg}^{-1}$ was infused over $4 \mathrm{~min}$; thereafter, remifentanil was infused at a maintenance rate of $0.25 \mu \mathrm{g} \cdot \mathrm{kg}^{-1} \cdot \mathrm{min}^{-1}$ throughout surgery. Group 3 (AG): a loading dose of alfentanil $50 \mu \mathrm{g} \cdot \mathrm{kg}^{-1}$ was infused over 4 min; thereafter, alfentanil was infused at a maintenance rate of $1 \mu \mathrm{g} \cdot \mathrm{kg}^{-1} \cdot \mathrm{min}^{-1}$ throughout surgery.

The initial infusion setting for propofol on the Diprifusor ${ }^{(B)}$ was a plasma concentration of $2 \mu \mathrm{g} . \mathrm{mL}^{-1}$ to be reached in $4 \mathrm{~min}$, thereafter the propofol infusion was set at a plasma concentration of $1 \mu \mathrm{g} \cdot \mathrm{mL}^{-1}$. If the patient was still responsive after this 4 min period, the Diprifusor ${ }^{(B)}$ setting remained at $2 \mu \mathrm{g} \cdot \mathrm{mL}^{-1}$ until the patient became unresponsive; thereafter, the Diprifusor ${ }^{\mathbb{B}}$ was set at $1 \mu \mathrm{g} \cdot \mathrm{mL}^{-1}$. When patients became unresponsive to verbal commands (failure to open the eyes on three consecutive requests), additional pancuronium was given to facilitate endotracheal intubation. Thereafter, patients' lungs were ventilated with oxygenenriched air without the addition of volatile anaesthetics.

Blood pressure and HR were registered at the following time intervals: $T_{1}$ : baseline; 5 min after insertion of the venous cannulae and radial artery cannula; $T_{2}$ : 4 min after the start of induction; $\mathrm{T}_{3}: 3 \mathrm{~min}$ after endotracheal intubation; $\mathrm{T}_{4}: 3 \mathrm{~min}$ after incision; $T_{5:}$ immediately before sternotomy; $T_{6}: 3$ min after sternotomy; $T_{7}$ : immediately after heparinization; at the time of an haemodynamic intervention (see below) and 2 min after this intervention. 
At the time of an alteration in study drug administration (opioid bolus dose or opofol targetcontrol adjustment) and 2 min after alterations in study drug adminration, an additional remifentanil bolus dose $1 \mu \mathrm{g} \cdot \mathrm{kg}^{-1}$ was administered in oups 1 and 2. An additional bolus dose of alfentanil $25 \mu \mathrm{g} \cdot \mathrm{kg}^{-1}$ was administered Group 3 if there were responses indicating inadequate anaesthesia with this infuin regimen: (a) systolic blood pressure $25 \mathrm{mmHg}$ above preinduction control; (b) HR above 90 beats $\mathrm{min}^{-1}$ in the absence of hypovolaemia (CVP $<6 \mathrm{mmHg}$ ); (c) her autonomic signs such as lachrymation, flushing or sweating, somatic responses cluding swallowing, coughing, eye opening, grimacing or bodily movements.

If the first dose of opioid was not fully effective within $2 \mathrm{~min}$, a second dose was ministered. If there was no effect within $2 \mathrm{~min}$, the target plasma concentration propofol was increased in $1 \mu \mathrm{g} \cdot \mathrm{mL}^{-1}$ steps until haemodynamic control was hieved. Once the patient's response was considered adequately controlled, the opofol infusion was decreased to the $1 \mu \mathrm{g} \cdot \mathrm{mL}^{-1}$ setting, which was the original lintenance period infusion rate.

Any hypotension, defined as a SAP below $80 \mathrm{mmHg}$ for more than $1 \mathrm{~min}$, curring during induction or during the maintenance of anaesthesia period, was ated with i.v. fluids, and, when indicated, incremental doses of ephedrine $2.5 \mathrm{mg}$ and/or dopamine infusion. Bradycardia, defined as a $\mathrm{HR}<40$ beats. min $^{-1}$ for re than $1 \mathrm{~min}$, was treated with atropine i.v.

Haemodynamic interventions were defined as: (a) administration of an extra lume load $\left(250 \mathrm{~mL}\right.$ of a colloid solution (Haemohes $6 \%{ }^{\circledR}$, B. Braun Medical, Oss, 1e Netherlands)); (b) administration of ephedrine; (c) administration of dopane; (d) administration of atropine.

Standard institutional hypothermic $\left(28^{\circ} \mathrm{C}\right)$ cardiopulmonary bypass (CPB) th cold cardioplegic solution cardiac arrest was performed in all patients. Before :aning from $\mathrm{CPB}$, patients were rewarmed to a rectal temperature of $35^{\circ} \mathrm{C}$. After a patient had arrived in the intensive care unit (ICU), the sedative- analgesic usions were continued for $4 \mathrm{~h}$ and then stopped. In Groups 1 and 2, an infusion remifentanil $0.025 \mu \mathrm{g}, \mathrm{kg}^{-1} \cdot \mathrm{min}^{-1}$ and in Group 3 an infusion of alfentanil $0.1 \mu \mathrm{g}$. ${ }^{-1} \cdot \mathrm{min}^{-1}$ was administered. Fifteen minutes before cessation of the sedative- analsic infusion, pirinitramide - a synthetic morphine derivative (analgesic potency comparison with morphine (=1) is 0.7$)-0.3 \mathrm{mg} \cdot \mathrm{kg}^{-1}$ i.v. was administered to the JRG and LDRG, and placebo was administered to the $A G$ in a blinded hion. ${ }^{15}$ Acetaminophen (paracetamol) $1 \mathrm{~g}$ rectally four times daily was started sen the patient arrived in the ICU.

Propofol was continued in the ICU at a rate of $0.5 \mathrm{mg} \cdot \mathrm{kg}^{-1} \cdot \mathrm{h}^{-1}$ and, if necessary, :reased to achieve the desired level of sedation (Ramsay sedation score 3, 4 or 16

The criteria for initiating the weaning process were haemodynamic stability, rmothermia (core temperature $>36.5^{\circ} \mathrm{C}$ ), chest tube drainage $<100 \mathrm{~mL} \cdot \mathrm{h}^{-1}$, no portant dysrhythmias, absence of shivering and acceptable ventilatory parameters 
during synchronous intermittent mandatory ventilation $\left(\mathrm{F}_{\mathrm{i}} \mathrm{O}_{2}<40 \%\right.$, peak endexpiratory pressure $<5 \mathrm{~cm} \mathrm{H}_{2} \mathrm{O}, \mathrm{PaO}_{2}>12 \mathrm{kPa}$ ).

When the patient was awake, oriented and cooperative and had started triggering the ventilator, the ventilator setting was switched to the pressuresupport mode. If the patient's triggering was adequate, the pressure support was gradually decreased, maintaining ventilatory parameters within acceptable limits (tidal volume $>5 \mathrm{~mL} \cdot \mathrm{kg}^{-1}$, vital capacity $>10 \mathrm{~mL} \cdot \mathrm{kg}^{-1}$, respiratory rate $>10$ breaths. $\mathrm{min}^{-1}$, minute ventilation $>90 \mathrm{~mL} \cdot \mathrm{kg}^{-1} \cdot \mathrm{min}^{-1}, \mathrm{FiO}_{2}<40 \%$, peak end-expiratory pressure $<5 \mathrm{~cm} \mathrm{H}_{2} \mathrm{O}, \mathrm{PaO}_{2}>12 \mathrm{kPa}$ ). If the patient could maintain these ventilatory parameters during $30 \mathrm{~min}$ of spontaneous respiration with the peak end-expiratory pressure set at $5 \mathrm{~cm} \mathrm{H}_{2} \mathrm{O}$, tracheal extubation was performed. Arterial blood-gas values were assessed before tracheal extubation, and at $30 \mathrm{~min}$ and $4 \mathrm{~h}$ after extubation. After this initial $4 \mathrm{~h}$ period in the ICU, pirinitramide $0.3 \mathrm{mg} \cdot \mathrm{kg}^{-1}$ intramuscularly (i.m.) was administered upon demand.

The primary efficacy variable of this study was the time of extubation: time from arrival in the ICU to extubation, and time from stopping the sedative infusion to extubation.

The secondary efficacy variables were:

- The haemodynamic stability associated with the anaesthetic management as measured by the haemodynamic data at $\mathrm{T}_{1}, \mathrm{~T}_{2}, \mathrm{~T}_{3}, \mathrm{~T}_{4}, \mathrm{~T}_{5}, \mathrm{~T}_{6}$ and $\mathrm{T}_{7}$ and by the number of haemodynamic interventions in the pre-bypass period.

- The suppression of intraoperative responses to noxious stimuli in the pre-bypass period as assessed by the number of extra bolus doses of the opioid and by the number of alterations in propofol plasma concentration settings.

- The quality of post-sedation pain management as measured by the number of opioid administrations in the ICU until discharge to a step-down unit.

The number of patients required for this study was based on an expected difference in the time of extubation; 75 patients (25 patients per treatment arm) were recruited to detect a difference of at least 1 standard deviation (SD) between both HDRG and LDRG, and the AG at $\alpha=0.025$ and $\beta 0.10$ (power $=0.9$ ).

\section{Statistical analysis}

Extubation times were compared among the three groups with the Kruskal-Wallis test. The number of haemodynamic interventions, of opioid-sedative interventions, and of postoperative pirinitramide administrations were compared among the groups using Fisher's exact test. For the haemodynamic data, baseline-adjusted means with their standard errors were estimated using a mixedmodel ANOVA with one common baseline value. The explanatory variables are: time (a within-patient factor with six levels: $\mathrm{T}_{2}-\mathrm{T}_{7}$ ), treatment group (a betweenpatient factor with three levels) and with the baseline $\left(T_{1}\right)$ measurement of the 


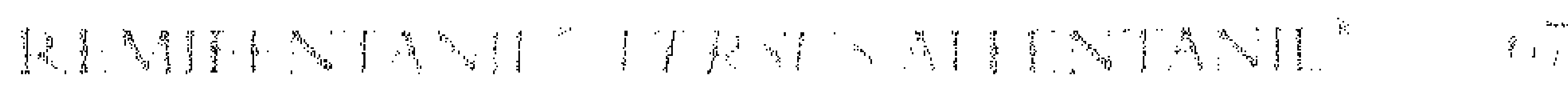

outcone variable considered as a co-variate. Also, the time-by-treatment interaction was tested.

\section{RESULTS}

Patient characteristics and surgical data are shown in Table 1.

\section{Extubation time}

No differences were observed between extubation times in the three groups (Table 2). Median extubation time after stopping the i.v. administration of sedativeopioid drugs was $300 \mathrm{~min}$ in the HDRG, $270 \mathrm{~min}$ in the LDR $G$ and $270 \mathrm{~min}$ in the $A G(P=0.606)$.

\section{Hacmodynamic stability}

Arterial pressure and $H R$ measured at $T_{1}, T_{2}, T_{3}, T_{4}, T_{5}, T_{6}$ and $T_{7}$ are shown in Figure 1. Significant time-by-treatment interactions were seen for $\mathrm{SAP}(\mathrm{P}=0.015)$, for MAP $(P=0.009)$ and for DAP $(P=0.0066)$. No significant interaction was seen for $\mathrm{HR}(\mathrm{P}=0.489)$. Also, no constant treatment effect was seen for $\mathrm{HR}(\mathrm{P}=$ $0.288)$. Time effects were highly significant $(P>0.01)$ for all haemodynamic variables considered. Figure 1 shows that $\mathrm{HR}$ remained stable in all groups throughout the study period. The induction of anaesthesia resulted in similar decreases in arterial pressure in the three groups. After endotracheal intubation, there was a similar, slight increase in arterial pressure in the various groups. Thereafter, arterial pressure remained stable and below baseline throughout the study period in HDRG; however, in LDRG and in the AG, arterial pressures increased and were higher than in HDRG from the start of surgery until the pre-cannulation surgical period.

The number of haemodynamic interventions in the pre-bypass period are shown in Table 3. Extra fluid was administered once in nine patients in HDRG, in five patients in LDRG and in four patients in AG. In one of the nine patients in the HDRG, a second bolus dose of extra fluid was given. Ephedrine $2.5 \mathrm{mg}$ intravenously was given in three patients in HDRG, in three patients in the LDRG and in four patients in AG. Atropine $0.5 \mathrm{mg}$ intravenously was used in one patient in AG. No dopamine was needed in any patient.

Atropine was administered for bradycardia after induction but before endotracheal intubation; most of the extra fluid and ephedrine was given in the preincision period in the absence of surgical stimulation. 
Table 1. Patients' characteristics and surgical data in patients undergoing propofol anaesthesia with either high-dose remifentanil, low-dose remifentanil or alfentanil for coronary artery surgery $(n=25$ patients)

\begin{tabular}{lccc}
\hline & HDRG & LDRG & AG \\
\hline Age $(\mathrm{yr})$ & $60.1 \pm 11.7$ & $60.2 \pm 9.4$ & $61.5 \pm 10.2$ \\
Weight $(\mathrm{kg})$ & $76.4 \pm 12.2$ & $83.2 \pm 13.3$ & $77.5 \pm 11.6$ \\
Surgery & & & \\
Number of distal anastomoses & $3.9 \pm 1.2$ & $3.6 \pm 1.1$ & $3.5 \pm 1.4$ \\
Duration of CPB (min) & $70.6 \pm 35.8$ & $72.9 \pm 29.76$ & $2.8 \pm 38.3$
\end{tabular}

Table 2. Extubation times.

\begin{tabular}{llll}
\hline & HDRG & LDRG & AG \\
\hline Median & 540 & 510 & 510 \\
Minimum & 360 & 240 & 340 \\
Maximum & 945 & 802 & 1050 \\
\hline
\end{tabular}

Extubation time is the time in minutes from entering the cardio-thoracic ICU until tracheal extubation; no significant differences were observed among the groups.

Table 3. The number of haemodynamic interventions in the pre-bypass period.

\begin{tabular}{lllll}
\hline & HDRG & LDRG & AG & $P$ \\
\hline Fluid & 9 & & & \\
$\quad$ Patients & 10 & 5 & 4 & 0.373 \\
$\quad$ Interventions & & 5 & 4 & \\
Ephedrine & 3 & & & \\
$\quad$ Patients & 3 & 3 & 4 & 1.000 \\
$\quad$ Interventions & & 3 & 4 & \\
Atropine & 0 & & & \\
$\quad$ Patients & 0 & 0 & 1 & 1.000 \\
$\quad$ Interventions & 0 & 0 & 1 & \\
\hline
\end{tabular}

Interventions: the number of fluid, ephedrine and atropine administrations in the pre-bypass period (fluid: administration of $250 \mathrm{~mL}$ of a colloid solution; ephedrine: a dose of $2.5 \mathrm{mg}$ ephedrine i.v.; atropine: a dose of $0.5 \mathrm{mg}$ atropine i.v.).

Table 4. The suppression of intraoperative responses to noxious stimuli in the pre-bypass period.

\begin{tabular}{lllll}
\hline & HDRG & LDRG & AG & P \\
\hline Opioid & & & & \\
$\quad$ Patients & 5 & 16 & 15 & 0.015 \\
$\quad$ Interventions & 9 & 30 & 27 & \\
Propofol & & & & \\
$\quad$ Patients & 1 & 4 & 2 & 0.516 \\
$\quad$ Interventions & 1 & 5 & 2 & \\
\hline
\end{tabular}

Interventions: the number of bolus doses of the opioid and alterations in propofol-TCI settings. 

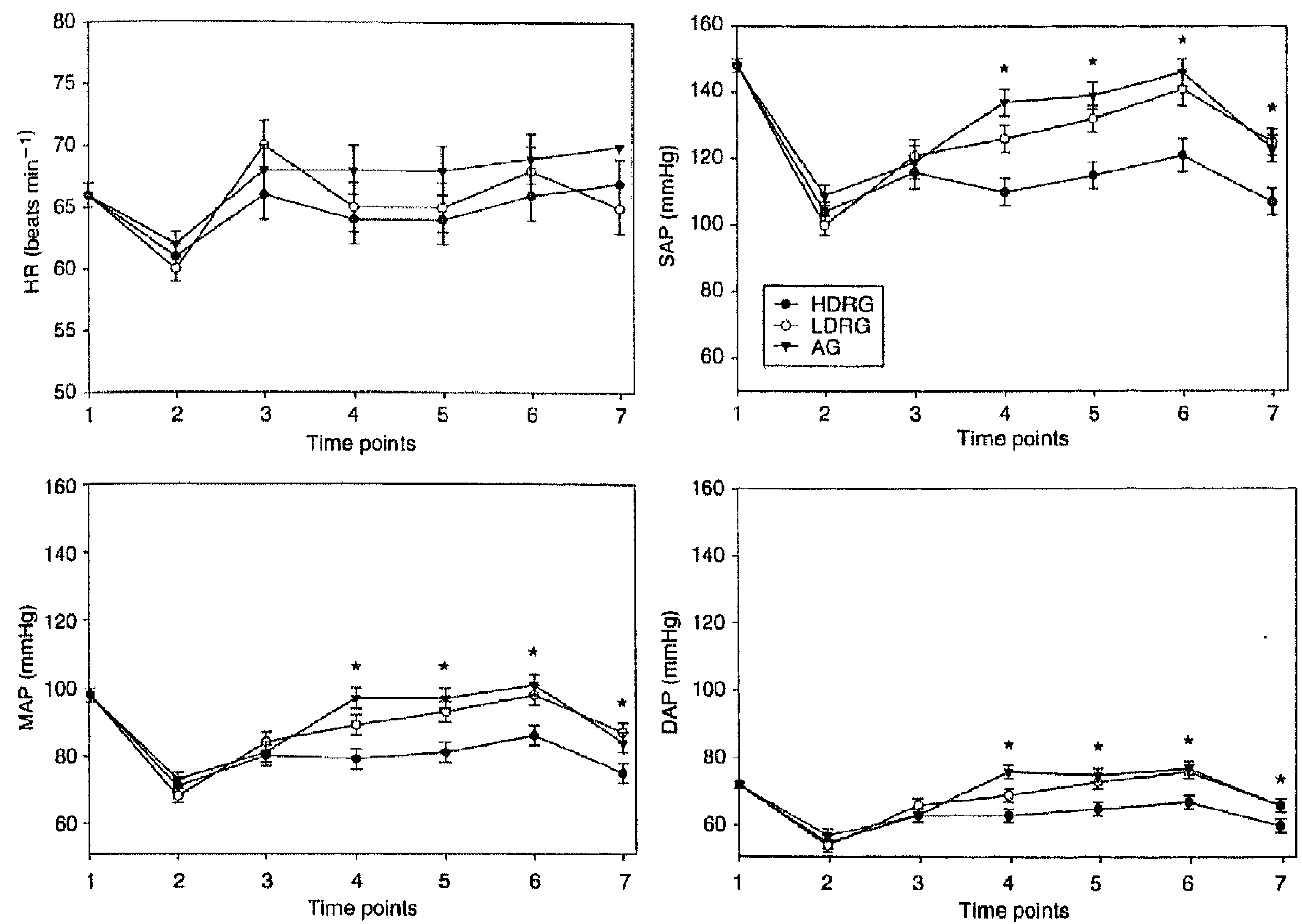

Figure 1. Haemodynamic effects of three different anaesthetic regimens in the pre-bypass period. Time points: 1 :baseline; $2: 4$ min after the start of induction; $3: 3 \mathrm{~min}$ after intubation; $4: 3$ min after incision; $5:$ immediately before sternotomy; $6: 3$ min after sternotony; $7:$ immediately after heparinization. Note that the scale for $\mathrm{HR}$ is from 50 to $80 \mathrm{beatsmin}^{-1}$ and that the scale for arterial pressure is from 50 to $160 \mathrm{~mm} \mathrm{Hg}$. Data are the mean $\pm \mathrm{SD}$. ${ }^{*}$ At these time points arterial pressures were significantly different between the three groups $(\mathrm{P}<$ $0.01)$.

The suppression of intraoperative responses to noxious stimuli in the pre-bypass period

The number of bolus doses of the opioid and of alterations in propofol-TCI settings are shown in Table 4. In HDRG, five patients required an extra bolus dose of the opioid. In LDRG, 16 patients were given an extra bolus of the opioid and in AG 15 patients $(P<0.015)$. An increase in the dose of propofol was necessary in one patient in HDRG, in four patients in the LDRG and in two patients in AG.

\section{Post-sedation analgesic requirements}

All patients remained overnight in the ICU. According to standard hospital procedures, patients who had undergone surgery in the morning hours were discharged from the ICU the next morning, patients who had undergone surgery in the afternoon were discharged from the ICU the following afternoon. Length of stay in the ICU was therefore similar in the three groups. 
Table 5. Post-sedation analgesic requirements.

\begin{tabular}{lllll}
\hline & HDRG & LDRG & AG & $P$ \\
\hline Pirinitramide & & & & \\
Patients & 11 & 12 & 18 & 0.118 \\
Interventions & 11 & 13 & 18 & \\
\hline
\end{tabular}

Interventions: the number of administrations of postoperative pirinitramide i.m.

During the patient's stay in the ICU pirinitramide i.m. was given to the patient when judged indicated by the nursing staff (VAS $>40 \mathrm{~mm}$ ). In HDR G, 11 patients received one dose of pirinitramide during their stay in the ICU. In LDRG, 12 patients received one dose of the opioid and one of these 12 patients received two doses. In AG, one dose of the opioid was given to 18 patients (Table 5).

There were no significant differences in the incidence of ischaemic injury in the three groups. None of the patients reported intraoperative recall.

\section{DISCUSSION}

In the present study, no differences in extubation time were observed among the three different anaesthetic techniques for coronary artery surgery. The administration of the long-acting opioid pirinitramide $15 \mathrm{~min}$ before stopping the propofol- remifentanil infusion did not prolong extubation time as compared to the propofol-AG and provided good postoperative analgesia. However, in the remifentanil $0.5 \mu \mathrm{g} \cdot \mathrm{kg}^{-1} \cdot \mathrm{min}^{-1}$ patient group, the suppression of haemodynamic responses to noxious stimuli was superior, as indicated by the significantly less additional opioid bolus doses required in this group. Also, in the remifentanil $0.5 \mu \mathrm{g}$. $\mathrm{kg}^{-1} \cdot \mathrm{min}^{-1}$ patient group, haemodynamic stability was superior, as indicated by the significant differences in blood pressures between the groups at the fixed measurement time points during surgery.

Total i.v. anaesthesia with a TCI of low-dose propofol has been described in cardiac surgery. ${ }^{17}$ The optimal target plasma propofol concentration is affected by the opioid with which propofol is combined and has not yet been defined. ${ }^{18} \mathrm{~A}$ propofol plasma concentration of $1.5-2 \mu \mathrm{g} \cdot \mathrm{mL}^{-1}$ has been used in cardiac surgery, without intraoperative recall. ${ }^{19}$

Due to their pharmacokinetic profile, both alfentanil and remifentanil are very suitable opioids to combine with propofol for cardiac anaesthesia when a fast postoperative recovery from surgery and anaesthesia is desired. An alfentanil infusion of $1 \mu \mathrm{g} \cdot \mathrm{kg}^{-1} \cdot \mathrm{min}^{-1}$, supplemented with additional boluses as necessary to the individual patient's requirements and immediately before surgical events that are known to be particularly stressful, has been described as a good anaesthetic technique for cardiac surgery. This technique was described as being probably superior to a technique which attempts to blunt all stress responses by the continuous administration of high infusion rates of the opioid without the use of boluses. ${ }^{3,20}$ 
The optimal dose of remifentanil infusion during cardiac surgery is still controersial. ${ }^{21}$ Infusions between 0.25 and $4 \mu \mathrm{g} \cdot \mathrm{kg}^{-1} \cdot \mathrm{min}^{-1}$ have been described. Severe ardiovascular depression with remifentanil has been reported. Perhaps infusion ttes too high (1-4 $\left.\mu \mathrm{g} \cdot \mathrm{kg}^{-1} \cdot \mathrm{min}^{-1}\right)$ for the surgical stimulus present, or the adminisation of bolus doses of remifentanil in the absence of surgical intervention could xplain, at least in part, this observed haemodynamic instability. In the present study, aemodynamic effects upon induction of anaesthesia were similar in the three roups, and during induction and surgery severe cardiovascular depression was not bserved. The minor decreases in arterial pressure were similar in the three groups ad could easily be treated with extra fluid and small doses of ephedrine. The need or additional bolus doses of the opioid or sedative was significantly less in the IDRG (P = 0.015). Therefore, in the present study, the haemodynamic response , stressful events was better controlled with remifentanil at an infusion rate of 0.5 $\mathrm{g} \cdot \mathrm{kg}^{-1} \cdot \mathrm{min}^{-1}$.

A major drawback of remifentanil, in this clinical setting, is the minimal residual nalgesia in the postoperative period. In the present study, remifentanil or alfentanil I combination with propofol were administered up until $4 \mathrm{~h}$ after the patient's rrival in the ICU. It has been reported that there is a lack of opioid tolerance uring remifentanil and alfentanil infusions for postoperative pain. ${ }^{22,23}$ irinitramide or placebo was administered in a blinded fashion i.v. 15 min before essation of the remifentanil infusion or of the alfentanil infusion, respectively. This zchnique provided good analgesia during the awakening and weaning period. Our ypothesis is that the administration of pirinitramide could explain, at least in part, thy the anticipated difference in extubation time between remifentanil and Ifentanil was not observed in the present study. Only in 11 patients in the HDRG ne dose of pirinitramide i.m. was given during the stay in the ICU vs. 18 patients I the AG. Patients were questioned about their pain sensations by the nursing staff t predetermined regular intervals on a scale from 'no pain' to 'slight pain' to 'modrate pain' to 'severe pain' to 'very severe pain'. Pirinitramide i.m. was administered on demand' whenever the patient's pain sensation was between slight and loderate.

In the present study, a delay of $4 \mathrm{~h}$ was chosen in the ICU before the cessation if the sedative-analgesic infusion to ensure the absence of bleeding, haemoynamic instability and hypothermia. Systemic normothermia with a core temperture of approximately $36.5^{\circ} \mathrm{C}$ is a key requirement for readiness of weaning and xtubation in post-cardiac surgical patients.

This study was not part of a fast-tracking protocol after cardiac surgery, as we elieve that efforts in fast tracking are better focused on the perioperative care of igh-risk patients rather than on methods to further reduce the already short duraion of postoperative ventilatory support in low-risk patients. ${ }^{24}$

In conclusion, in this study no differences were found in extubation times ,etween remifentanil/pirinitramide- and alfentanil-based total i.v. anaesthetic tech- 
niques using a TCI of propofol. Remifentanilbased cardiac anaesthesia was associated with superior suppression of intraoperative responses to noxious stimuli and resulted in superior haemodynamic stability. Good postoperative analgesia could easily be obtained by the administration of a long-acting opioid before cessation of the infusion of remifentanil. Further investigations are warranted to compare the use of intraoperative remifentanil and different strategies of postoperative pain management on postoperative recovery characteristics.

\section{Acknowledgements}

We would like to thank the CTICU nurses and the nurse anaesthetists for their help and participation in this study. 


\section{REFERENCES}

1. Phillips AS, McMurray TJ, Mirakhur RK, Gibson FM, Elliott P. Propofol-fentanyl anesthesia: a comparison with isoflurane-fentanyl anesthesia in coronary artery bypass grafting and valve replacement surgery. J Cardiothorac Vasc Anesth 1994; 8: 289-296.

2. Hall RI, Murphy JT, Landymore R, Pollak PT, Doak G, Murray M. Myocardial metabolic and hemodynamic changes during propofol anesthesia for cardiac surgery in patients with reduced ventricular function. Anesth Analg 1993; 77:680-689.

3. Roekaerts PMHJ, Gerrits HJ, Timmerman BE, De Lange S. Continuous infusions of alfentanil and propofol for coronary artery surgery. J Cardiothorac Vasc Anesth 1995; 9: 362-367.

4. Glass PSA, Hardman D, Kamiyama Y, et al. Preliminary pharmacokinetics and pharmacodynamics of an ultrashort- acting opioid: remifentanil (G187084B). Anesth Analg 1993; 77: 1031-1040.

5. Gray JM, Kenny GN. Development of the technology for 'Diprifusor' TCI systems. Anaesthesia 1998; 53 (Suppl 1): 22-27.

6. Sebel PS, Lowdon JD. Propofol: a new intravenous anesthetic. Anesthesiology 1989;71:260-277.

7. Kapila A, Glass PSA, Jacobs JR, et al. Measured contextsensitive half-times of remifentanil and alfentanil. Anesthesiology 1995; 83: 968-975.

8. Burkle H, Dunbar S, Van Aken H. Remifentanil: a novel, short-acting, i-opioid. Anesth Analg 1996; 83: 646-651.

9. Bowdle TA, Ready LB, Kharasch ED. Transition to postoperative epidural or patient-controlled intravenous analgesia following total intravenous anaesthesia with remifentanil and propofol for abdominal surgery. Eur J Anaesthesiol 1997; 14:374-379.

10. Albrecht S, Schuttler J, Yarmush J. Postoperative pain management after intraoperative remifentanil. Anesth Analg 1999; 89: S40-S45.

11. Fletcher D, Pinaud M, Scherpereel P, Clyti N, Chauvin M. The efficacy of intravenous 0.15 vs. $0.25 \mathrm{mg} / \mathrm{kg}$ intraoperative morphine for immediate postoperative analgesia after remifentanilbased anesthesia for major surgery. Anesth Analg 2000; 90: 666-671.

12. Yarmush J, D'Angelo R, Kirkhart B, et al. A comparison of remifentanil and morphine sulfate for acute postoperative analgesia after total intravenous anesthesia with remifentanil and propofol. Anesthesiology 1997; 87: 235-243.

13. Elliott P, O'Hare R, Bill KM, Phillips AS, Gibson FM, Mirakhur RK. Severe cardiovascular depression with remifentanil. Anesth Analg 2000; 91:58-61.

14. Kazmaier S, Hanekop GG, Buhre W, et al. Myocardial consequences of remifentanil in patients with coronary artery disease. Br J Anaesth 2000;84: 578-583.

15. Kumar N, Rowbotham DJ. Piritramide. Br J Anaesth 1999; 82: 3-5.

16. Ramsay MA, Savege TM, Simpson BR, Goodwin R. Controlled sedation with alphaxalone-alphadolone. BMJ 1974; 2:656-659.

17. Barvais L, Rausin I, Glen JB. Administration of propofol by target-controlled infusion in patients undergoing coronary artery surgery.J Cardiothorac Vasc Anesth 1996; 10:877-883.

18. Vuyk J, Mertens MJ, Olofsen E, Burm AG, Bovill JG. Propofol anesthesia and rational opioid selection. Anesthesiology 1997; 87: 1549-1562.

19. Olivier P, Sirieix D, Dassier P, D'Attellis N, Baron JF. Continuous infusion of remifentanil and target-controlled infusion of propofol for patients undergoing cardiac surgery: a new approach for scheduled early extubation. J Cardiothorac Vasc Anesth 2000; 14:29-35.

20. Philbin DM, Rosow CE, Schneider RC, Koski G, D'Ambra MN. Fentanyl and sufentanil anesthesia revised: how much is enough? Anesthesiology 1990; 73:5-11.

21. Hogue CWJ, Bowdle TA, O'Leary C. A multicenter evaluation of total intravenous anesthesia with remifentanil and propofol for elective inpatient surgery. Anesth Analg 1996;83:279-285.

22. Schraag S, Checketts MR, Kenny GNC. Lack of rapid development of opioid tolerance during alfentanil and remifentanil infusions for postoperative pain. Anesth Analg 1999; 89: 753-757. 
23. Cortinez LI, Brandes V, Munoz HR, Guerrero ME, Mur M. No clinical evidence of acute opioid tolerance after remifentanil-based anaesthesia. Br J. Anaesth 2001; 87: 866-869.

24. Coniat P, Beaussier M. Fast tracking after coronary artery bypass graft surgery. Anesth Analg 2001; 92: 1081-1083. 


\section{CHAPTER 6}

Comparison of the modulatory effects of four different fast-track anesthetic techniques on the inflammatory response to cardiac surgery with cardiopulmonary bypass

J. Heijmans ${ }^{1}$, E. Fransen ${ }^{2}$, W. Buurman ${ }^{3}$, J. Maessen $^{2}$, and P. Roekaerts ${ }^{1}$

University Hospital Maastricht, Departments of ${ }^{1}$ Anaesthesiology,

${ }^{2}$ Cardio-thoracic Surgery and ${ }^{3}$ Surgery.

Journal of Cardiothoracic and Vascular Anesthesia. Vol 21, No.4 (August), 2007: pp 512-518: 
7) MHTER

\section{ABSTRACT}

Objectives: To compare the modulatory effects of four anesthesia techniques on the inflammatory response to cardiac surgery.

Design: Prospective, randomized, comparative study.

Setting: Major university teaching hospital.

Participants: Sixty patients undergoing elective surgery.

Interventions: Patients were randomised into an alfentanil group, a high-dose remifentanil group, a low-dose remifentanil group or a thoracic-epidural group, in combination with a propofol-target controlled infusion. The study was blinded for the opioid, except in the epidural group. Tight control of perioperative hemodynamic parameters was maintained and the postoperative management was strictly standardised. Bactericidal permeability increasing protein as an indicator of the polymorphonuclear neutrophil response, interleukin-6 as an inducer of the acute phase response, and lipopolysaccharide binding protein and C-reactive protein as parameters of the acute phase response, were determined at regular intervals. Ventilator dependency and analgesia were evaluated as clinical outcome measures.

Measurements and Main Results: Interleukin-6 levels increased in all groups. Plasma levels in the epidural group were significantly higher at all timepoints than in the other groups. The increase in the plasma levels of bactericidal permeability increasing protein, lipopolysaccharide binding protein and C-reactive protein showed the same pattern in all groups and no significant differences between the four groups were observed.

Conclusions: Supplementation of a fast-track anesthetic technique with epidural analgesia preserves hemodynamic stability and is associated with faster extubation times and less postoperative pain. Furthermore, thoracic epidural analgesia, by less dampening of the IL-6 response to cardiac surgery as compared to a TIVA anesthestic technique, helps to maintain the inflammatory response to cardiac surgery. 


\section{INTRODUCTION}

Cardiac surgery provokes a vigorous inflammatory response which persists into the postoperative period. ' Factors that activate the inflammatory response include surgical trauma, blood transfusion, and hypothermia. In addition, cardiopulmonary bypass (CPB) may activate the inflammatory response via contact activation of the immune system following exposure of blood to the foreign surfaces of the CPBcircuit, via ischemia-reperfusion injury after release of the aortic cross-clamp and via splanchnic hypoperfusion allowing gut translocation of endotoxin. ${ }^{2}$ Factors influencing the incidence, severity, extent, and clinical outcome of the inflammatory response to cardiac surgery are numerous and include preoperative patient disorders, surgical factors, extracorporeal perfusion factors, transfusion factors and postoperative factors like continuous renal replacement therapies and mechanical ventilation. 3 However, the reasons why certain patients develop life-threatening perioperative complications while others do not, are currently not well understood. The contribution of the inflammatory response to patient outcome is potentially remediable and therefore deserves attention. Diverse techniques, including maintenance of hemodynamic stability, minimization of exposure to CPB circuitry, and pharmacologic and immunomodulatory agents have been studied by several investigators as well as in our institution. ${ }^{3-6}$ Recently, drugs and techniques used to produce anesthesia and maintain postoperative sedation and analgesia are thought to possess immunomodulatory effects. ${ }^{1,7-11}$ However, data on the effects of anesthesia management on the inflammatory response to cardiac surgery are lacking.

Therefore, we designed the present descriptive study to compare the potential modulatory effects of four different modern anesthesia techniques on the inflammatory response. We measured bactericidal permeability increasing protein (BPI) as an indicator of the polymorphonuclear neutrophil (PMN) response, interleukin-6 (IL-6) as an inducer of the acute phase response, and lipopolysaccharide binding protein (LBP) and C-reactive protein (CRP) as parameters of the acute phase during anesthesia and during the first 18 hours postoperatively. All groups received a total intravenous anesthetic technique using target-controlled infusion (TCI) of propofol in combination with, in group 1 the opioid alfentanil; in group 2 the opiod remifentanil in a high dose; in group 3 remifentanil in a low dose; and in group 4 remifentanil in combination with thoracic epidural analgesia. Intraoperative and postoperative management was otherwise similar in both groups according to a strict protocol which also included a sedation protocol. Because hemodynamic instabilities are known to affect the inflammatory response, hemodynamic parameters were tightly controlled within narrow limits and detailed hemodynamic measurements were performed. Ventilator dependency and analgesia were evaluated as clinical outcome measures. 


\section{METHODS}

The study was approved by our hospital's Medical Ethics Committee and written informed consent was obtained from 60 patients who were randomised into an alfentanil group (AG), a high-dose remifentanil group (HDRG), a low-dose remifentanil group (LDRG) or a thoracic-epidural group (TEG), in combination with a propofol-TCI technique. The study was blinded for the opioid infusion, except in the thoracic epidural group. Exclusion criteria included left ventricular ejection fraction of less than $25 \%$, hypothermic circulatory arrest, recent myocardial infarction, preoperative inotropic or intraaortic balloon pump support, emergency surgery and significant pulmonary, endocrine, metabolic, or neurological diseases. All patients were receiving chronic beta-adrenoceptor blocking drugs. The distribution of other medications affecting cardiovascular variables (calcium antagonists, ACE inhibitors, nitrates) was the same in all groups. All these other medications were stopped the evening before surgery. Patients were premedicated with oral midazolam 0.1-0.2 mg.kg, administered together with their individual betaadrenceptor blocking drug.

For the thoracic-epidural group, the epidural catheter (Arrow, FlexTipPlus, $19 \mathrm{G}$ ) was inserted at least more than one hour before surgery at the C7-T1 level by a median approach and hanging drop technique. A test dose of $2 \mathrm{~mL}$ of lidocaine $2 \%$ was given to test for the correct position of the catheter. A loading dose of 10 $\mathrm{mL}$ bupivacaine $0.25 \%$ with $2.5 \mathrm{mg}$ morphine was infused over one hour. National guidelines for neuraxis blockade and anticoagulation were respected concerning the insertion of the thoracic epidural catheter.

Upon arrival in the operation room, patients received $7.5 \mathrm{~mL} . \mathrm{kg}^{-1}$ of a glucosesalt containing infusion and baseline hemodynamic data (T1) were obtained: heart rate (HR), systolic, mean and diastolic arterial pressure (SAP, MAP, DAP). During the induction of anesthesia, this cristalloid solution was administered to maintain cardiac filling pressures at their normal values (central venous pressure (CVP) between 6 and $12 \mathrm{~mm} . \mathrm{Hg}$ ). After preoxygenation and baseline hemodynamic measurements, a precurarization dose of pancuronium $20 \mu \mathrm{g} \cdot \mathrm{kg}^{-1}$ was administered to the patient. Immediately after this, both the alfentanil (Rapifen, Janssen Pharmaceutica, Beerse, Belgium) or remifentanil (Ultiva, GlaxoSmithKline, Zeist, The Netherlands) and propofol infusions were administered with the BD Master Diprifusor TCI system (software developed by the University of Glasgow, UK). Four groups of patients were compared. Group 1 (AG): a loading dose of alfentanil $50 \mu \mathrm{g} \cdot \mathrm{kg}^{-1}$ was infused over $4 \mathrm{~min}$, thereafter, alfentanil was infused at a maintenance rate of $1 \mu \mathrm{g} \cdot \mathrm{kg}^{-1} \cdot \mathrm{min}^{-1}$ throughout surgery; group 2 (HDRG): a loading dose of remifentanil $2.5 \mu \mathrm{g} \cdot \mathrm{kg}^{-1}$ was infused over $4 \mathrm{~min}$, thereafter, remifentanil was infused at a maintenance rate of $0.5 \mu \mathrm{g} \cdot \mathrm{kg}^{-1} \cdot \mathrm{min}^{-1}$ throughout surgery; group 3 (LDRG): a loading dose of remifentanil $2.5 \mu \mathrm{g} \cdot \mathrm{kg}^{-1}$ was infused over 4 min, thereafter, remifentanil was infused at a maintenance rate of $0.25 \mu \mathrm{g} \cdot \mathrm{kg}^{-1} \cdot \mathrm{min}^{-1}$; and 
group 4 (TEG): a loading dose of remifentanil $2.5 \mu \mathrm{g} \cdot \mathrm{kg}^{-1}$ was infused over 4 min, thereafter, remifentanil was infused at a maintenance rate of $0.125 \mu \mathrm{g} \cdot \mathrm{kg}^{-1} \cdot \mathrm{min}^{-1}$ and via thoracic epidural infusion bupivacaine $0.375 \%$ plus morphine $0.2 \mathrm{mg} \cdot \mathrm{mL}^{-1}$ was administered at a rate of $1.5 \mathrm{~mL} \cdot \mathrm{h}^{-1}$ throughout surgery. The initial infusion setting for propofol on the Diprifusor was a plasma concentration of $2 \mu \mathrm{g} \cdot \mathrm{mL}^{-1}$ to be reached in $4 \mathrm{~min}$, there after the propofol infusion was set at a plasma concentration of $1 \mu \mathrm{g} \cdot \mathrm{mL}^{-1}$. If the patient was still responsive after this $4 \mathrm{~min}$ period, the Diprifusor setting remained at $2 \mu \mathrm{g} \cdot \mathrm{mL}^{-1}$ until the patient became unresponsive; thereafter, the Diprifusor was set at $1 \mu \mathrm{g} \cdot \mathrm{mL}^{-1}$. When patients became unresponsive to verbal commands (failure to open the eyes on three consecutive requests), additional pancuronium was given to facilitate endotracheal intubation. Thereafter, patients' lungs were ventilated with oxygen-enriched air without the addition of volatile anesthetics.

Blood pressure and $\mathrm{HR}$ were registered at the following time intervals: $\mathrm{T} 1$ : baseline, 5 min after insertion of the venous cannulae and radial artery cannula; T2: 4 min after the start of induction; T3: 3 min after endotracheal intubation; T4: 3 min after incision; T5: immediately before sternotomy; T6:3 min after sternotomy; T7: immediately after heparinisation; at the time of an hemodynamic intervention (see below) and 2 min after this intervention, at the time of an alteration in study drug administration (opioid bolus dose or propofol TCI adjustment) and 2 min after alterations in study drug administration. An additional bolus dose of alfentanil $25 \mu \mathrm{g} \cdot \mathrm{kg}^{-1}$ was administered in group 1 and an additional remifentanil bolus dose 1 $\mu \mathrm{g} \cdot \mathrm{kg}^{-1}$ was administered in groups 2,3 and 4 , if there were responses indicating inadequate anesthesia with this infusion regimen: (a) systolic blood pressure 25 mmHg above preinduction control; (b) a HR above 90 beats.min ${ }^{-1}$ in the absence of hypovolemia (CVP $<6 \mathrm{mmHg}$ ); (c) other autonomic signs such as lachrymation, flushing or sweating, somatic responses including swallowing, coughing, eye opening, grimacing or bodily movements. If the first dose of opioid was not fully effective within $2 \mathrm{~min}$, a second dose was administered. If there was no effect within $2 \mathrm{~min}$, the target plasma concentration of propofol was increased in 1 $\mu$ g.mL $\mathrm{m}^{-1}$ steps until hemodynamic control was achieved. Once the patients' response was considered adequately controlled, the propofol infusion was decreased to the $1 \mu \mathrm{g} \cdot \mathrm{mL}^{-1}$ setting, which was the original maintenance period infusion rate. Any hypotension, defined as a SAP below $80 \mathrm{mmHg}$ for more than $1 \mathrm{~min}$, occurring during induction or during the maintenance of anesthesia period, was treated with i.v. fluids, and, when indicated, incremental doses of ephedrine $2.5 \mathrm{mg}$ i.v. and/or norepinephrine infusion. Bradycardia, defined as a $\mathrm{HR}<40$ beats/min for more than $1 \mathrm{~min}$, was treated with atropine i.v. Hemodynamic interventions were defined as: (a) administration of an extra volume load $(250 \mathrm{~mL}$ of a colloid solution (Haemohes 6\%, B. Braun Medical, Oss, The Netherlands)); (b) administration of ephedrine; (c) infusion of norepinephrine; (d) administration of atropine. The hemodynamic stability associated with the anesthetic management was measured 
by the hemodynamic measurements at the predetermined timepoints and by the number of hemodynamic interventions in the pre-bypass period. The suppression of intraoperative responses to noxious stimuli in the pre-bypass period was assessed by the number of extra bolus doses of the opioid and by the number of alterations in propofol plasma concentration settings.

At arrival in the ICU, a sedative-analgesic infusion of propofol $0.5 \mathrm{mg} \cdot \mathrm{kg}^{-1} \cdot \mathrm{h}^{-1}$ together with, in group 1 alfentanil $0.1 \mu \mathrm{g} \cdot \mathrm{kg}^{-1} \cdot \mathrm{min}^{-1}$ and in groups 2,3 and 4 remifentanil $0.025 \mu \mathrm{g} \cdot \mathrm{kg}^{-1}$. $\mathrm{min}^{-1}$, was started for 4 hours. If necessary, propofol was increased to achieve the desired level of sedation (Ramsay sedation score 3,4 or 5). Additionally, acetaminophen $1 \mathrm{~g}$ four times daily was started as a basic analgesic. In the TEG group, the catheter was left in position 48 hours postoperatively and bupivacaine $0.125 \%$ and morphine $0.2 \mathrm{mg} \cdot \mathrm{mL}^{-1}$ were infused at at rate 1.5 $\mathrm{mL} \cdot \mathrm{h}^{-1}$. Fifteen minutes before cessation of the sedative-analgesic infusion, pirinitramide - a synthetic morphine derivate (analgesic potency in comparison with morphine (=1) is 0.7$)-0.15 \mathrm{mg} \cdot \mathrm{kg}^{-1}$ i.v. was administered in the remifentanil groups 2 and 3 , and placebo was administered to the AG in a blinded fashion.

After stopping the infusion, patients were questionned about their pain sensations by the nursing staff at predetermined regular intervals on a scale from "no pain" to "slight pain" to "moderate pain" to "severe pain" to "very severe pain". Piritramide $0.3 \mathrm{mg} \cdot \mathrm{kg}^{-1}$ intramuscularly (i.m.) was administered "on demand" whenever the patient's pain sensation was between slight and moderate. The quality of post-sedation pain management was measured by the number of opioid administrations in the ICU until discharge to a step-down unit. The criteria for initiating the weaning process were hemodynamic stability, normothermia (core temperature $>36.5^{\circ} \mathrm{C}$ ), chest tube drainage $<100 \mathrm{~mL} . \mathrm{h}^{-1}$, no important dysrhythmias, absence of shivering and acceptable ventilatory parameters $\left(\mathrm{FiO}_{2}<40 \%\right.$, peak end-expiratory pressure $<5 \mathrm{~cm} \mathrm{H}_{2} \mathrm{O}, \mathrm{PaO}_{2}>12 \mathrm{kPa}$ ). When the patient was awake, oriented and cooperative, and had started triggering the ventilator, the ventilator setting was switched to the pressure-support mode. If the patient's triggering was adequate, the pressure support was gradually decreased, maintaining ventilatory parameters within acceptable limits (tidal volume $>5 \mathrm{~mL} . \mathrm{kg}$, vital capacity $>10 \mathrm{~mL} . \mathrm{kg}$, respiratory rate $>10$ breaths $/$ min, minute ventilation $>90$ mL.kg.min, $\mathrm{FiO}_{2}<40 \%$, peak end-expiratory pressure $<5 \mathrm{~cm} \mathrm{H}_{2} \mathrm{O}, \mathrm{PaO}_{2}>12 \mathrm{kPa}$ ). If the patient could maintain these ventilatory parameters during $30 \mathrm{~min}$ of spontaneous respiration with the peak end-expiratory pressure set at $5 \mathrm{~cm} \mathrm{H}_{2} \mathrm{O}$, tracheal extubation was performed. Extubation times were recorded.

Cardiopulmonary bypass was standardized and performed using moderate hypothermia, a hollow-fiber membrane oxygenator (Capiox SX 18, Terumo Europe N.V. Leuven, Belgium), a venous reservoir (JVR 1900, Jostra AG, Hirrlingen, Germany), and an arterial line filter (Pall LeukoGuard LGG, Pall, England). The Capiox SX18 oxygenator is L-coated, all other components exposed to blood were pretreated with heparin-bonded coating (Bioline coating, Jostra). 
The standard priming of the extracorporeal circuit included gelofusine (B. Braun, Melsungen, AG, D-34209 Melsungen, Germany), mannitol 20\% and human llbumin 20\%. Mean arterial blood pressure was maintained between 70 and 90 $\mathrm{nmHg}$, in some patients by titrating continuous infusions of small doses of either ;odium nitroprusside or phenylephrine.

Blood samples for BPI, IL-6 and LBP were taken at five timepoints. After induction of anesthesia (T1), at the end of surgery (T2), four hours after arrival in the ICU (T3), eight hours after arrival (T4) and 18 hours after arrival (T5). Plasma levels of BPI, IL-6 and LBP were measured using sandwich enzymelinked immunosorbent assays (ELISAs). ${ }^{+}$Blood samples for C-reactive protein (CRP) were taken the day before surgery and on day one, two, four and six postoperatively. The CRP concentrations were measured using a turbidimetric method.

\section{Statistical Analysis}

Differences in clinical characteristics were compared using analysis of variance (ANOVA) with Bonferroni as post hoc test. To compare extubation times, a non parametric test, the Kruskal-Wallis test, was used. The General Linear Model (GLM) repeated measures analysis of variance was used to investigate the effects of four different anesthetic techniques on inflammatory mediators and hemodynamic parameters. Analyses were performed with group (four groups) as between subject factor, and time (five timepoints for the inflammatory mediators and seven timepoints for the hemodynamic data) as within subject factor. In case of significant difference, the Bonferroni post hoc test was applied. Sample size was determined using data from our laboratory for IL-6, and indicated that an enrollment of 15 patients per group would provide $80 \%$ power in detecting $100 \%$ change in IL-6. ${ }^{4}$ Differences were considered significant at p-values less than 0.05 . All data are presented as mean \pm standard error of the mean.

\section{RESULTS}

Patient characteristics and surgical data were similar in both groups (Table 1). Also, the total amount of blood transfusion was the same in the four groups and all groups were hemodynamic stable without inotropic support during the whole study and there were no complications necessitating prolonged hospitalisation (data not shown). In the TEG group, the extent of the neurogenic blockade was from thoracic sensory level 2 to thoracic sensory level 10 in all patients.

The intraoperative hemodynamic stability associated with the four different anesthetic techniques is shown in figure 1 and table 2 . Overall hemodynamic stability was excellent with minor, although significant, differences between the four groups. Heart rates were similar at all time points and mean arterial blood pressures were also similar at timepoints 1,2 and 7. At timepoint 3, mean arterial blood 

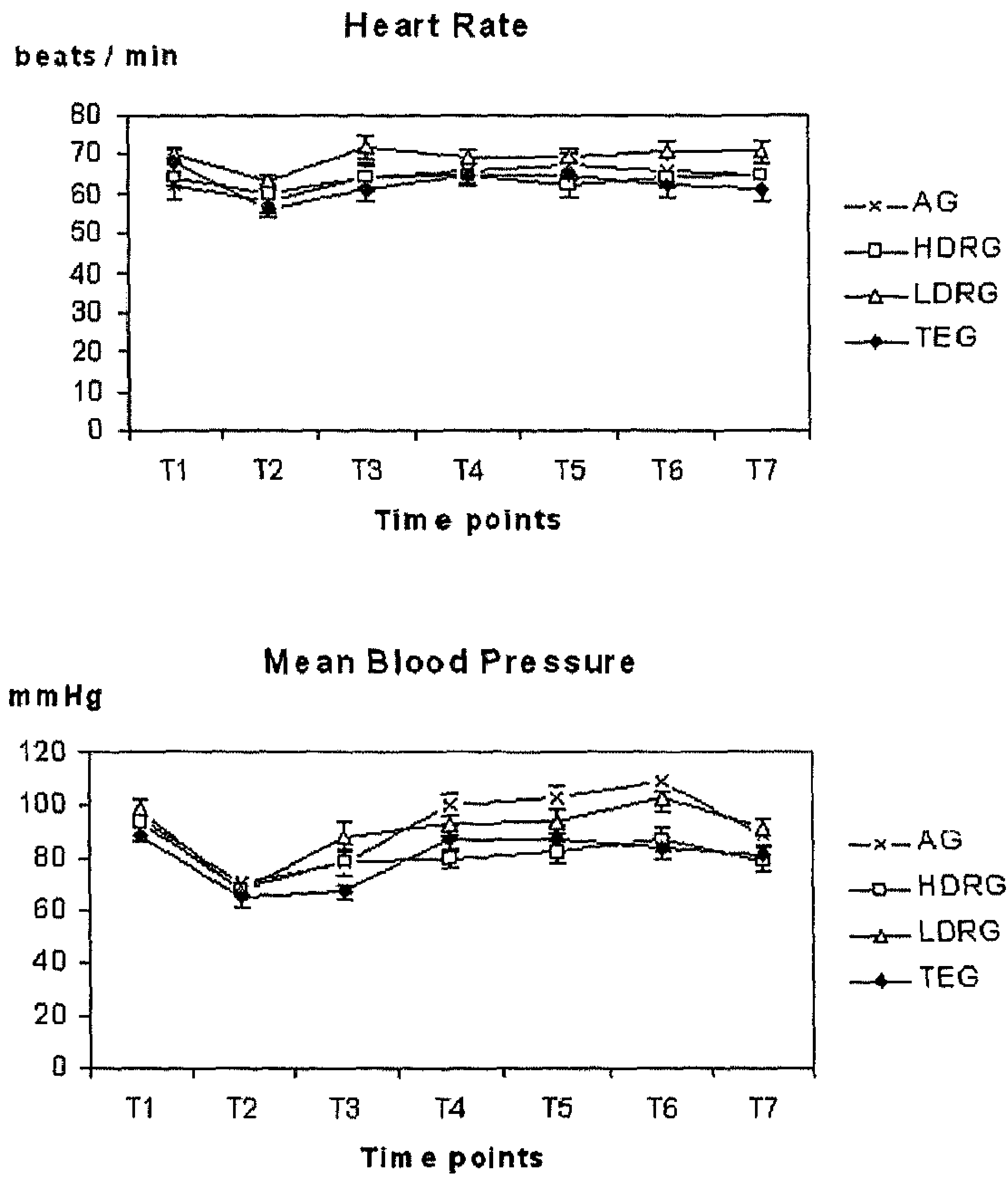

Figure 1. Heart Rate and Blood Pressure for 60 patients in four different anesthesia groups and at seven different timepoints; $A G=$ alfentanil group;HDR $G=$ high-dose remifentanil group; $L D R G=$ low-dose remifentanil group; TE $G$ = thoracic epidural group; $\mathrm{T} 1=$ baseline, $5 \mathrm{~min}$ after insertion of the venous cannulae and radial artery cannula; $T 2=4 \mathrm{~min}$ after the start of induction; $\mathrm{T} 3=3 \mathrm{~min}$ after endotracheal intubation; $\mathrm{T} 4=3$ min after incision; $\mathrm{T} 5=$ immediately before sternotomy; $\mathrm{T} 6=3 \mathrm{~min}$ after sternotomy; $\mathrm{T} 7=$ immediately after heparinization; Significant differences were observed at T3: TEG versus $L D R G$ ( $P=0.007)$, at T4: HDRG versus $A G(P=0.01)$, at $T 5: H D R G$ versus $A G(P=0.009)$ and at $T 6$ :TEG versus $L D R G(P=0.02)$, TEG versus $A G(P=0.001)$ and $H D R G$ versus $A G(P=0.006)$.

Table 1. Clinical Characteristics

\begin{tabular}{lccccc}
\hline & AG & HDRG & LDRG & TEG & P \\
\hline Age (years) & $61 \pm 10$ & $57 \pm 11$ & $61 \pm 9$ & $61 \pm 10$ & NS \\
Anastomoses $(\mathrm{n})$ & $3 \pm 2$ & $4 \pm 1$ & $3 \pm 1$ & $4 \pm 1$ & NS \\
CPB time (min) & $72 \pm 26$ & $80 \pm 31$ & $79 \pm 20$ & $74 \pm 23$ & NS \\
X-clamp time (min) & $51 \pm 23$ & $57 \pm 25$ & $51 \pm 14$ & $46 \pm 17$ & NS \\
\hline
\end{tabular}

Data are mean \pm SEM. NS $=$ no significant difference. Four patient groups $(n=15$ in each group): $A G=$ alfentanil anesthesia; HDRG: high-dose remifentanil anesthesia; LDR G: low-dose remifentanil anesthesia; TEG: thoracic epidural anesthesia; $\mathrm{CPB}=$ cardiopulmonary bypass; $\mathrm{X}$-clamp: aortic cross clamp. 

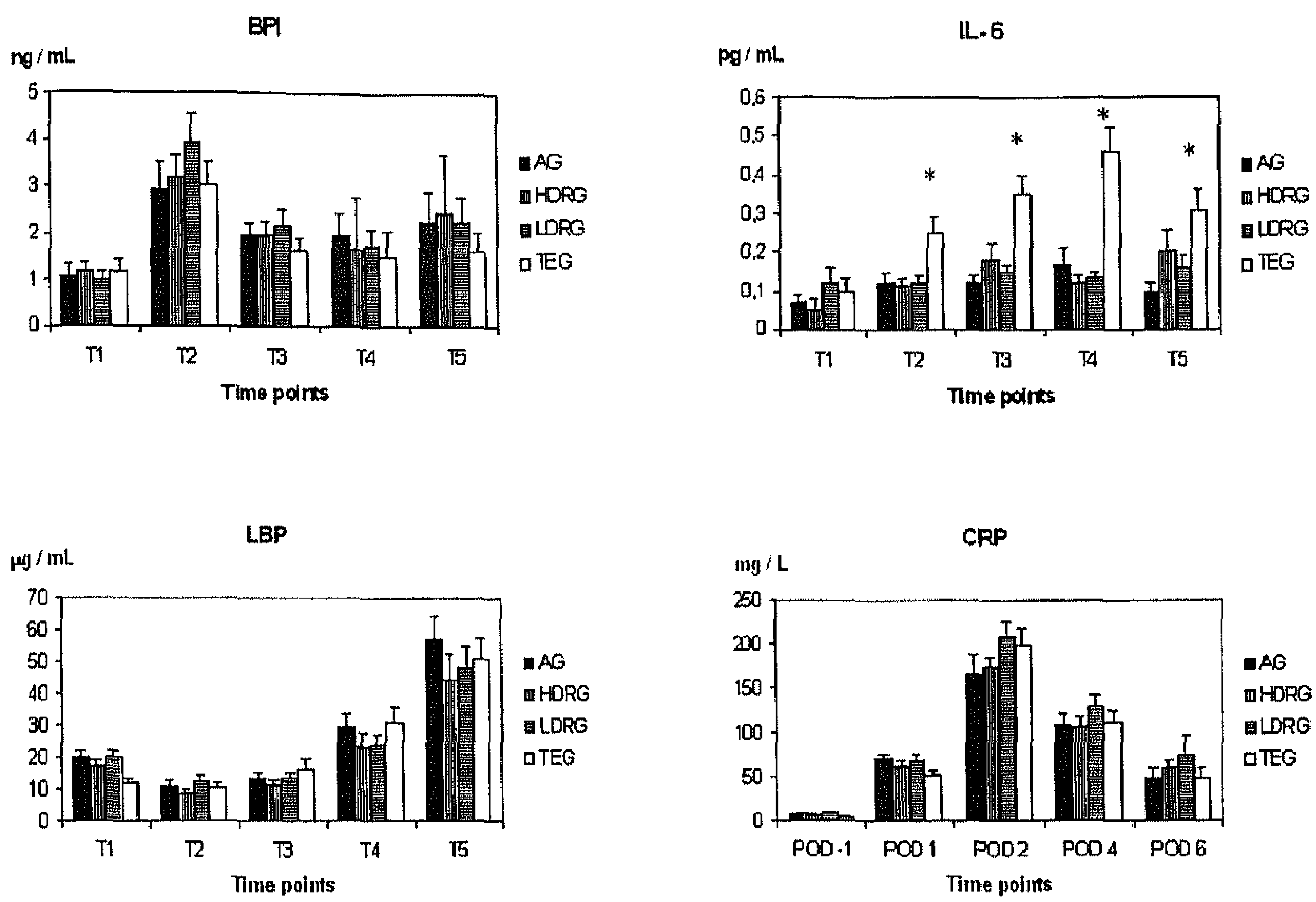

Figure 2, Plasma levels for 60 patients of bactericidal permeability increasing protein, interleukin-6, lipopolysaccharide binding protein and C-reactive protein in four different anesthesia groups and at five different timepoints; $A G=$ alfentanil group; $H D R G=$ high-dose remifentanil group; $L D R G=$ low-dose remifentanil group; $\mathrm{TEG}=$ thoracic epidural group; $\mathrm{T} 1=$ after induction of anesthesia; $\mathrm{T} 2=$ at the end of surgery; $\mathrm{T} 3=$ four hours after arrival in the ICU; T4 = eight hours after arrival in the ICU; $T 5=18$ hours after arrival in the ICU; $\mathrm{POD}=$ post operative day; Significant differences were observed in the IL- 6 group at T2: TEG versus AG, LDR $G$ and HDR $G(P=0.01)$, at T3: TEG versus $A G, L D R G$ and HDRG $(P=0.01)$, at T4: TEG versus $A G$, LDRG and HDRG $(P<0.000)$, and at T5: TEG versus AG, LDRG and HDRG $(P=0.006)$.

pressure was lower in the TEG group as compared to the LDRG. At timepoints 4 and 5, the MAP was lower in the HDRG as compared to the AG. At timepoint 6, the MAP was lower in the TEG as compared to the AG and the LDRG and the MAP was also lower at this timepoint in the HDRG as compared to the AG. In table 2 is shown that there was no difference between the groups concerning the administration of ephedrine, atropine and extra bolus doses of propofol; in the HDRG more fluids and less opioid bolus doses had to be given as compared to the three other groups to achieve optimal hemodynamic stability.

In table 3, postoperative ICU data are shown. The post-sedation analgesic requirement, as an indicator of postoperative stress, was similar in the $A G$, the LDRG and the HDRG. In the TEG, no additional opioids had to be administered in the postoperative period. The median extubation time from stopping the sedative infusion until extubation, was comparable in the four groups, although patients in the TEG were extubated faster than in the AG and HDRG. 
Table 2. Perioperative Clinical Management

\begin{tabular}{lccccc}
\hline Patients $(\mathbf{n})$ & AG & HDRG & LDRG & TEG & P \\
\hline Fluid & 1 & $6^{\star}$ & 3 & 0 & $\mathrm{P}=0.007$ HDRG vs TEG \\
Ephedrine & 1 & $7^{\star}$ & 3 & 1 & $\mathrm{P}=0.016$ HDRG vs TEG \\
Atropine & 1 & 0 & 0 & 1 & $\mathrm{NS}$ \\
Opioid & 9 & $4^{\star}$ & 9 & 7 & $\mathrm{P}=0.007$ HDRG vs TEG \\
Propofol & 3 & 0 & 2 & 0 & $\mathrm{NS}$ \\
\hline
\end{tabular}

$\star \mathrm{P}=<0.05$; Four patient groups ( $\mathrm{n}=15$ in each group): $\mathrm{AG}=$ alfentanil anesthesia; HDRG: high-dose remifentanil anesthesia; LDRG: low-dose remifentanil anesthesia; TEG: thoracic epidural anesthesia.

Table 3. Postoperative ICU data

\begin{tabular}{lccccc}
\hline & AG & HDRG & LDRG & TEG & $P$ \\
\hline Piritramide (n patients) & 10 & 7 & 10 & $0^{\star}$ & $P=0.045$ HDRG vs TEG \\
Median Extubation time & 505 & 510 & 450 & $375^{\star}$ & $P=0.003$ HDRG vs TEG \\
\hline
\end{tabular}

$\star \mathrm{P}=<0.05$; Four patient groups $(\mathrm{n}=15$ in each group): $\mathrm{AG}=$ alfentanil anesthesia; HDR $\mathrm{G}$ : high-dose remifentanil anesthesia; LDR G: low-dose remifentanil anesthesia; TEG: thoracic epidural anesthesia.

A similar pattern in changes of the plasma levels of the measured inflammatory mediators at the different time points was observed in the four experimental patient groups.

Baseline levels of BPI were the same in all groups (Fig 2). BPI levels in all groups were elevated from the first postbypass measurement until the first postoperative day, reaching peak levels at timepoint T2. At this timepoint, BPI levels were significantly elevated in all groups. At timepoint T3, plasma levels were significantly higher in the AG, LDRG and HDRG. At timepoint T5, only the LDRG was still significant from baseline.Baseline levels of IL-6 were the same in all groups (Fig 3). IL-6 plasma levels increased in all groups, but no significance was reached in the LDRG. In the AG, IL-6 plasma levels were significantly higher than their baseline value at time points T3 and T4. Increases in IL-6 in the HDRG and in the TEG were significant at all timepoints. The plasma levels of IL-6 in the thoracic epidural group were significantly higher at all timepoints than in the other three general anesthesia groups.

Baseline levels of LBP were the same in all groups (Fig 4). Mean plasma levels of LBP were initially decreased at timepoints $T 2$ and $T 3$, reaching significance in the AG and in the HDRG. Plasma levels were significantly increased in the AG, LDRG and TEG at time point $T 4$, and significantly increased in all groups on the first postoperative day. No significant differences were found between the four groups at any timepoint. Baseline levels of CRP were the same in all groups (Fig 5). There was a similar and significant increase in CRP in all groups at all time points, reaching a peak plasma concentration on the second postoperative day. No significant differences were found between the four groups at either timepoint. 


\section{DISCUSSION}

The present study was performed to compare the effect of four different anesthetic techniques on the inflammatory response to cardiac surgery with cardiopulmonary bypass in patients where the perioperative management was strictly standardised. The release pattern of all measured inflammatory mediators was the same in all patient groups. No significant differences in the plasma levels of BPI, LBP, and CRP were observed between the four study groups. However, IL-6 levels were significantly higher at all timepoints in the patient group where thoracic epidural analgesia was part of the otherwise total intravenous anesthesia technique as compared to the other three patient groups where no epidural technique was applied.

The development of strategies to dampen the overexpressed, detrimental neuro-endocrine response to stress is of utmost importance, as an uncontrolled inflammatory response plays a significant role in the morbidity and mortality observed in patients undergoing cardiac surgery with CPB. Recent studies show that propofol and opioids posess potentially important immunomodulatory effects 1.2 , but controlled studies comparing the effects of anesthesia management with different new opioids on the inflammatory response to cardiac surgery are lacking. The hypothesis underlying the present study was that the choice of opioid and/or timing of administration and/or dosing of the opioid could influence the degree and magnitude of the subsequent inflammatory response and its consequences. ${ }^{12}$ With the increasing emphasis on fast-tracking the cardiac surgical patient, total intravenous anesthesia with propofol in combination with thoracic epidural analgesia and shorter-acting narcotics are gaining more popularity. Therefore, we compared the short-acting alfentanil with different doses of the very potent remifentanil, the latest fentanyl analog available for clinical use. Thoracic epidural analgesia supplemented anesthesia in the lowest dose remifentanil group. Remifentanil is the first nonspecific esterase-metabolized opiate. Rapid onset, rapid recovery, and lack of accumulation make it an ideal anesthetic. ${ }^{13}$ Furthermore, in the present study, the entire postoperative management in the intensive care unit was very strictly standardized the first 24 hours postoperatively by the research protocol to minimize the effects of varying somatic conditions on the inflammatory response. In our study, three groups differed solely in the opioid or dose of opioid they received during anesthesia.

Modern total intravenous anesthesia (TIVA) techniques using propofol in combination with alfentanil or remifentanil in different doses have similar effects on the cytokine release to cardiac surgery. In the thoracic epidural group, plasma levels of IL- 6 were higher at all timepoints as compared to the three general anesthesia groups. This is a surprising finding, because in several previous studies epidural anesthesia did not affect the cytokine response. ${ }^{8,9,11}$ Epidural analgesia combined with general anesthesia did not influence the IL-6 response to pelvic 
surgery compared with general anesthesia, ${ }^{1+}$ and the same was found in upper abdominal surgery. ${ }^{15}$ The cytokine and inflammatory response using either a highdose opioid fentanyl anesthetic technique or a combination of thoracic epidural analgesia with bupivacaine and low-dose fentanyl in combination with midazolam and enflurane showed no difference between the groups with regard to cytokine response. ${ }^{11}$ Consistent with these findings, it was reported that thoracic epidural anesthesia for cardiac surgery is not associated with a reduction in cytokine release. ${ }^{16}$ The cytokine response and also the magnitude of the IL-6 release depends on the surgical intervention, the amount of blood transfusions and duration of extracorporeal circulation. ${ }^{5}$ These factors were the same in all groups. However, recent evidence suggests that epidural anesthesia can potentially affect the inflammatory response to surgery. For instance, epidural analgesia blunts the postsurgical lymphocyte depression, which is important in infection resistance. ${ }^{8,17}$ The effect of epidural anesthesia on several cytokines which are key players in the innate immune response has been reported in a few studies. In one of these studies in CABG patients, epidural anaesthesia decreased circulating levels of the anti-inflammatory cytokine IL-10 but did not affect IL $-6 .^{18}$ In another study, epidural analgesia suppressed the pro-inflammatory cytokine IL-6 release from in vitro stimulated monocytes compared to opioid analgesia. ${ }^{17}$

The reason for elevated levels of IL-6 during epidural anesthesia as compared to remifentanil anesthesia remains unclear, but may be explained by one of the following hypotheses. First, this could be due to the less intense sympatholytic effect of remifentanil anesthesia as compared to epidural anesthesia. It was reported that a high sympathetic tone inhibits the inflammatory response. ${ }^{19}$ However, we did not measure plasma cartecholamines in the present study. Secondly, in the epidural group, morphine was injected into the cervical epidural space, and it has been reported that central activation of opioid receptors from morphine results in an increase in IL-6 through autonomic activation of the adrenal cortex. ${ }^{20}$ In accordance, in pediatric surgical patients perioperative release of IL-6 was higher after caudal sufentanil. ${ }^{21}$ A third explanation could be that remifentanil itself may decrease IL-6 plasma concentration. Opioids have multiple effects on the immune system, mediated indirectly via the central nervous system or through direct interactions with the $\mu 3$ opioid receptors on cells of the immune system. ${ }^{1,22}$ In noncardiac surgery, total intravenous anesthesia with alfentanil and propofol diminished the IL -6 response to abdominal surgery as compared to volatile anesthesia with isoflurane. ${ }^{24}$ The authors suggested that this reduction was mediated by alfentanil via opioid receptors on monocytes. Remifentanil also seems to blunt parts of the cellular immune response. ${ }^{25}$ However, there are no reports studying the effects of remifentanil on the IL-6 response. There is one study in CABG patients anesthetized with propofol and remifentanil in which IL-6 concentrations were also measured. In this study, plasma concentrations of $60 \mathrm{pg} \cdot \mathrm{mL}^{-1}$ were measured at the end of surgery, which is comparable with our results. In contrast, in other studies in 
CABG patients with different long-acting opioid-based anesthetic techniques, IL-6 plasma concentrations between 200 and $500 \mathrm{pg} \cdot \mathrm{mL}^{-1}$ were measured. ${ }^{4,11,18}$

The important concept of peri-operative cytokine modulation by anesthetic agents is exemplified by IL-6. This pro-inflammatory cytokine is released in response to major surgery, ${ }^{25,26}$ and is known to be associated with postoperative morbidity, ${ }^{27-30}$ with a reported reduction in mortality of septic shock in response to treatment with monoclonal antibodies to IL- 6 or IL- 6 receptor. ${ }^{30}$ The clinical implications of this difference in IL-6 response remain uncertain, as the immune system is complex and difficult to assess from individual tests. A first implication could be the increased likehood for adverse outcome after central neuraxial analgesia. However, in a recent meta-analysis, no differences in the rates of mortality or myocardial infarction after $C A B G$ surgery were found with epidural analgesia. ${ }^{31}$ Also, the association between IL-6 and adverse outcome seems to be only valid in the presence of an overwhelming systemic release, such as in sepsis. ${ }^{32}$ Moreover, immunological complications after cardiac surgery appear to be mainly linked to an insufficient production of anti-inflammatory cytokines instead of an overproduction of pro-inflammatory cytokines. ${ }^{33}$ Nevertheless, the decision to insert a thoracic epidural in a patient for cardiac surgery should be made very carefully. Until the added risk of epidural hematoma formation is not quantified, the riskbenefit ratio of this technique should be assessed for each individual patient. A second implication for the less dampened IL-6 response during epidural anesthesia is a potential improved resistance to infection, because this pro-inflammatory protein produced by macrophages, monocytes and endothelial cells is necessary for initiating an effective inflammatory response against infection. In a meta-analysis, less wound infections were described in patients having neuro-axial anesthesia. ${ }^{34}$ In the current study, there were no infectious complications in neither group although our study was underpowered to investigate such an effect.

A limitation is that this study could not be blinded completely for obvious reason, which could have influenced the results on detubation time and on pain measurement. However, the moment of detubation and administration of analgesics were strictly protocolised and performed by nurses not involved with the study. Another limitation is that IL- 6 concentrations but not IL- 6 activity was measured which is considered a limitation of most studies on IL-6. ${ }^{35}$

In conclusion, we demonstrated that the supplementation of a fast-track anesthetic technique with epidural analgesia preserves hemodynamic stability and is associated with faster extubation times and less postoperative pain. Numerous other benefits for epidural analgesia in CABG surgery have been cited. ${ }^{36}$ This study focused on a potential immunomodulatory beneficial effect of thoracic epidural analgesia in this clinical setting. We described that thoracic epidural analgesia, by less dampening of the IL-6 response to cardiac surgery as compared to a TIVA anesthestic technique, helps to maintain the inflammatory response to cardiac surgery. Thus, thoracic epidural anesthesia is in many ways an ideal technique for 
fast-tracking in cardiac surgery. However, until the added risk of a spinal cord compression has not been quantified, the decision to insert a thoracic epidural catheter should be made very carefully. 


\section{REFERENCES}

1. Laffey JG, Boylan JF, Cheng DCH: The systemic inflammatory response to cardiac surgery. Anesthesiology 97:215-252,2002

2. Levy JH, Tanaka KA: Inflammatory response to cardiopulmonary bypass. Ann Thorac Surg 75 : S715 - S720, 2003

3. Hall RI, Smith MS, Rocker G: The systemic inflammatory response to cardiopulmonary bypass: Pathophysiological, Therapeutic, and Pharmacological Considerations. Anesth Analg 85: 766782, 1997

4. Fransen E, Maessen J, Dentener M, et al: Systemic inflammation present in patients undergoing CABG without extracorporeal circulation. Chest 113:1290 - 1295, 1998

5. Fransen $E$, Maessen J, Dentener $M$, et al: Impact of blood transfusion on inflammatory mediator release in patients undergoing cardiac surgery. Chest 116:1233 - 1239, 1999

6. Fransen EJ, Maessen JG, Hermens WT, et al: Peri-operative myocardial tissue injury and the release of inflammatory mediators in coronary artery bypass graft patients. Cardiovasc Res 45 : $853-859,2000$

7. Schneemilch CE, Ittenson A, Ansorge S, et al: Effect of 2 anesthetic techniques on the postoperative proinflammatory and anti-inflammatory cytokine response and cellular immune function to minor surgery. J Clin Anesth 17:517 - 527,2005

8. Volk T, Schenk M, Voigt K, et al: Postoperative epidural anesthesia preserves lymphocyte, but not monocyte, immune function after major spine surgery. Anesth Analg 98: 1086 - 1092, 2004

9. Yokoyama M, Itano $\mathrm{Y}$, Katayama $\mathrm{H}$, et al: The effects of continuous epidural anesthesia and analgesia on stress response and immune function in patients undergoing radical esophagectomy. Anesth Analg 101: 1521 - 1527, 2005

10. Hashimoto T, Hashimoto S, Hori $Y$, et al: Epidural anaesthesia blocks changes in peripheral lymphocytes subpopulation during gastrectomy for stomach cancer. Acta Anaesthesiol Scand 39: $294-298,1995$

11. Brix-Christensen V, Tonnesen E, Sorensen IJ, et al: Effects of anaesthesia based on high versus low doses of opioids on the cytokine and acute-phase protein responses in patients undergoing cardiac surgery. Acta Anaesthesiol Scand 42:63-70, 1998

12. Abraham E: Physiologic stress and cellular ischemia: Relationship to immunosuppression and susceptibility to sepsis. Ciit Care Med 19:613-618, 1991

13. Heijmans JH, Maessen JG, Roekaerts PM: Remifentanil provides better protection against noxious stimuli during cardiac surgery than alfentanil. Eur J Anaesthesiol 21:612 - 618,2004

14. Moore $\mathrm{CM}$, Desborough JP, Powell $\mathrm{H}$, et al: Effects of extradural anaesthesia on interleukin-6 and acute phase response to surgery. Br J Anaesth 72:272 - 279, 1994

15. Naito Y, Tamai S, Shingu K, et al: Responses of plasma adrenocorticotropic hormone, cortisol, and cytokines during and after upper abdominal surgery. Anesthesiology 77:426 - 431, 1992

16. Ganapathy S, Murkin JM, Dobkowski W, et al: Stress and inflammatory response after beating heart surgery versus conventional bypass surgery: The role of thoracic epidural anesthesia. Heart Surg Forum 4:323-327,2001

17. Beilin $B$, Shavit $Y$, Trabekin $E$, et al: The effects of postoperative pain management on immune response to surgery. Anesth Analg 97:822 - 827, 2003

18. Volk T, Dopfiner UR, Schmutzler M, et al: Stress induced IL-10 does not seem to be essential for early monocyte deactivation following cardiac surgery. Cytokine 24:237-243, 2003

19. Sternberg EM: Neural regulation of innate immunity: a coordinated non-specific host response to pathogens. Nat Rev Immunol 6:318 - 328, 2006

20. Houghtling RA, Bayer BM: Rapid elevation of plasma interleukine- 6 by morphine is dependent on autonomic stimulation of adrenal gland. J Pharmacol Exp Ther 300:213-219, 2002

21. Bichel T, Rouge J-C, Schlegel S, et al: Epidural sufentanil during paediatric cardiac surgery: effects on metabolic response and postoperative outcome. Paediatr Anaesth 10:609-617,2000 
22. Bidlack JM: Detection and function of opioid receptors on cells from the immune system. Clin Diagn Lab Immunol 7:719-723,2000

23. Crozier TA, Muller JE, Quittkat D, et al: Effect of anaesthesia on the cytokine responses to abdominal surgery. Br J Anaesth 72:280 - 285, 1994

24. Sacerdote P, Gaspanil L, Rossoni G: Effect of the opioid remifentanil on cellular immune response in the rat. Int Immunopharmacol 1:713 - 719, 2001

25. Cruickshank AM, Fraser WD, Burns HJ, et al: Response of serum interleukin-6 in patients undergoing elective surgery of varying severity. Clin Sci (Lond) 79:161-165, 1990

26. Goerlach $G$, Holzheimer R, Nimitz K, et al: Behaviour of interleukin- 6 in patients undergoing cardiopulmonary bypass. J Cardiovasc Surg 33: 66, 1992

27. Hall GM, Desborough JP: Interleukin-6 and the metabolic response to surgery. $\mathrm{Br}$ J Anaesth 69: $337-338,1992$.

28. Ertel W, Faist E, Nestle C, et al: Kinetics of interleukin-2 and interleukin-6 synthesis following major mechanical trauma.J Surg Res 48:622-628, 1990

29. Oka Y, Murata A, Nishijima J, et al: Circulating interleukin-6 as a useful marker for predicting postoperative complications. Cytokine 4:298-304, 1992

30. Libert C, Vink A, Coulie P, et al: Limited involvement of interleukin-6 in the pathogenesis of lethal septic shock as revealed by the effect of monoclonal antibodies against interleukin- 6 or its receptor in various murine models. Eur J Immunol 22: 2625 - 2630, 1992

31. Liu SS, Block BM, Wu CL: Effects of perioperative central neuraxial analgesia on outcome after coronary artery bypass surgery: A meta-analysis. Anesthesiology 101: 153-161, 2004

32. Pinsky MR, Vincent J-L, Deviere J, et al: Serum cytokine levels in human septic shock. Relation to multiple-system organ failure and mortality. Chest 103: 565 - 575, 1993

33. Nathan N, Preux PM, Feiss P, et al: Plasma interleukin-4, interleukin-10, and interleukin-13 concentrations and complications after coronary artery bypass surgery. J Cardiothorac Vasc Anesth 14:156-160,2000

34. Rodgers $A$, Walker $N$, Shug $S$, et al: Reduction of postoperative mortality and morbidity with epidural or spinal anaesthesia: results from overview of randomised trials. BMJ 321:1 - 12,2000

35. Song M, Kellum JA: Interleukin-6. Crit Care Med 33 (12 Suppl): S463 - S465, 2005

36. Kamming $D, D$ avis $W$ : Thoracic epidural analgesia for coronary artery surgery. A bridge too far? Eur J Anaesthesiol 22: $85-88,2005$ 


\section{CHAPTER 7}

\section{Pulmonary function and inflammatory markers in patients undergoing coronary revascularization with or without cardiopulmonary bypass}

J.H. Heijmans ${ }^{1}$, K.S.A.E. Liem ${ }^{1}$, G.M.C. Damoiseaux ${ }^{2}$, J.G. Maessen ${ }^{3}$, P.M.H.J. Roekaerts ${ }^{1}$

\footnotetext{
${ }^{1}$ Department of Anaesthesiology. ${ }^{2}$ Department of Clinical and Experimental Immunology. ${ }^{3}$ Department of Cardiothoracic Surgery
} 


\section{SUMMARY}

Lung injury after cardiac surgery is believed to result from cardiopulmonary bypass and its pro-inflammatory effects. To test this hypothesis, we compared the oxygenation ratios, the extravascular lung water indices and the systemic and pulmonary TNF- $\alpha$ and IL- 8 at predetermined intervals in coronary artery surgery patients with or without cardiopulmonary bypass. No differences in oxygenation ratios and in extravascular lung water indices were found. Serum values of TNF- $\alpha$ and IL-8 increased in both groups but were higher in the cardiopulmonary bypass group (end of surgery: mean (SD) TNF- $\alpha 3.68$ (2.5) vs 2.20 (1.2) pg. $\mathrm{ml}^{-1}(\mathrm{P}=0.043$ [CI 0.05-2.9]) and mean (SD) IL-8 19.45 (10.8) vs 6.31 (5.3) pg.m ${ }^{-1}(\mathrm{P}=0.001$ [CI 6.9-19.3]). In bronchoalveolar lavage fluid, TNF- $\alpha$ and IL-8 increased in both groups with no differences between the groups. 


\section{INTRODUCTION}

Pulmonary dysfunction after cardiac surgery with cardiopulmonary bypass (CPB) is an important cause of postoperative morbidity.' The aetiology of this pulmonary impairment is believed to be multifactorial, occurring as a result of the combined effects of anaesthesia, surgery and CPB. CPB activates inflammatory processes, resulting in damage to lung parenchyma and increased pulmonary capillary permeability. ${ }^{2,3}$

We therefore hypothesized that cardiac surgery with cardiopulmonary bypass ( $\mathrm{CPB}$ group), may be associated with more postoperative pulmonary dysfunction than cardiac surgery without CPB (off-pump group). Recent studies comparing pulmonary dysfunction after cardiac surgery with or without $C P B$ have produced conflicting evidence. However markers of systemic and/or pulmonary inflammation were not measured in any of these studies. ${ }^{4-8}$ These markers would provide evidence as to whether differences in lung dysfunction between the two groups are due to the inflammatory effects of $\mathrm{CPB}$ or to other interventional differences.

The aim of this prospective study was to assess the inflammatory effect of CPB on postoperative pulmonary dysfunction. To compare the magnitude of pulmonary dysfunction, the oxygenation ratios and extravascular lung water indices were measured in two groups of patients undergoing coronary artery surgery with or without $\mathrm{CPB}$. The inflammatory effects were assessed using the pro-inflammatory mediators, IL-8 and TNF- $\alpha$, in blood and bronchoalveolar lavage (BAL) fluid.

\section{METHODS}

Approval of the hospital ethics committee and patients' written informed consent was obtained for this prospective study of 20 patients undergoing coronary artery surgery with cardiopulmonary bypass (CPB group) and 20 patients undergoing coronary artery surgery without CPB (off-pump group). Exclusion criteria included left ventricular ejection fraction $<30 \%$, recent myocardial infarction, preoperative inotropic or intra-aortic balloon pump support, emergency surgery and significant pulmonary, endocrine, metabolic, or neurological diseases. All patients were receiving chronic beta-adrenoceptor blocking drugs. The distribution of other medications affecting cardiovascular variables was the same in the two groups. Patients were premedicated with midazolam together with their individual anti-anginal medication.

Upon arrival in the operation room patients received $7.5 \mathrm{ml} . \mathrm{kg}^{-1}$ of a solution containing $\mathrm{NaCl} 0.3 \%$ and Glucose 3.3\% (Baxter B.V., NL-3542 Utrecht, Netherlands). During induction of anaesthesia, this crystalloid solution was administered to maintain central venous pressure (CVP) between 6 and $12 \mathrm{mmHg}$. After preoxygenation, a precurarisation dose of pancuronium $20 \mu \mathrm{g} \cdot \mathrm{kg}^{-1}$ was adminis- 
tered. Immediately thereafter alfentanil $50 \mu \mathrm{g} \cdot \mathrm{kg}^{-1}$, etomidate $0.3 \mathrm{mg} \cdot \mathrm{kg}^{-1}$ and pancuronium $80 \mu \mathrm{g} \cdot \mathrm{kg}^{-1}$ were administered. Maintenance of anaesthesia was with alfentanil $2 \mu \mathrm{g} \cdot \mathrm{kg}^{-1} \cdot \mathrm{min}^{-1}$ and propofol $2 \mathrm{mg} \cdot \mathrm{kg}^{-1} \cdot \mathrm{hr}^{-1}$ supplemented, if necessary, with bolus doses of alfentanil or propofol.

\section{Anaesthetic management in the $C P B$ group}

Before connecting the extracorporeal circuit for CPB, heparin 300 IU. $\mathrm{kg}^{-1}$ (Heparin Leo, Leo Pharmaceutical Products BV, Weesp, The Netherlands) was administered to achieve an activated coagulation time (ACT) greater than 480 . The extracorporeal circuit consisted of a hollow-fiber membrane oxygenator (Capiox SX 18, Terumo Europe N.V. Leuven, Belgium), a venous reservoir (JVR 1900, Jostra AG, Hirrlingen, Germany) and arterial line filter (Pall LeukoGuard LGG, Pall, England). The Capiox SX18 oxygenator was L-coated; all other components exposed to blood were pretreated with a heparin-bonded coating (Bioline coating, Jostra). The standard priming of the extracorporeal circuit was $1300 \mathrm{mll}$ of Gelofusin (B. Braun, Melsungen, AG, D-34209 Melsungen, Germany), $200 \mathrm{ml}$ mannitol 20\%,100 ml human albumin $20 \%, 50 \mathrm{ml} \mathrm{NaHCO} 38.4 \%, 20 \mathrm{ml} \mathrm{KCL}$ 7.45\% (B. Braun Medical BV, Uden, The Netherlands), and 6500 IU heparin.

After institution of $\mathrm{CPB}$ at a flow rate of $2.41 \mathrm{~min}^{-1}$ per square meter and after a blood temperature of $34^{\circ} \mathrm{C}\left(32.0\right.$ to $\left.36.0^{\circ} \mathrm{C}\right)$ had been reached, the heart was topically cooled with $4^{\circ} \mathrm{C} 0.9 \%$ saline solution until it fibrillated. The aorta was then crossclamped, and a single dose of approximately $800 \mathrm{ml}(600$ to $1000 \mathrm{ml})$ of St. Thomas No. 1 cardioplegic solution at $4.0^{\circ} \mathrm{C}$ was infused into the aortic root over a 4 minute period to provide myocardial preservation. Topic cooling was maintained during the infusion of the cardioplegic solution. Target flow rates of 2.4 l. $\mathrm{min}^{-1} \cdot \mathrm{m}^{-2}$ were maintained through all periods of CPB. Pulsatile perfusion was used during the period of aortic crossclamping. Mean arterial blood pressure was maintained between 70 and $90 \mathrm{mmHg}$.

In all patients, the left anterior descending coronary artery was revascularized with the left internal mammary artery. Papaverine was locally applied to the mammary artery after dissection to prevent spasm. After completion of all the distal anastomoses, the aortic crossclamp was removed and the proximal anastomosis was performed with a partial occlusion clamp after spontaneous or electrical defibrillation, while rewarming of the patient to $36^{\circ} \mathrm{C}$ continued.

After $\mathrm{CPB}$ and at the end of surgery, heparin was reversed by $3 \mathrm{mg} \cdot \mathrm{kg}^{-1}$ protamine chloride (Hoffman-La Roche BV, Mijdrecht, The Netherlands). Reversal of the heparin effect was determined by heparin-protamine titration (titration assay cartridges, Medtronic HemoTec, Inc., Englewood, Colo.) on the Hepcon System-Four (Medtronic HemoTec). All pump blood was returned to the 
patient through the aortic cannula or via infusion bags without haemoconcentration.

\section{Anaesthetic management in the off-pump group}

The anaesthetic technique was the same as in the CPB group. Heparin 150 IU. $\mathrm{kg}^{-1}$ was given before surgery to achieve an ACT of greater than 250 seconds. After surgery, the heparin effect was reversed with $1.5 \mathrm{mg} \cdot \mathrm{kg}^{-1}$ protamine and checked with the Hepcon system.

Exposure of the heart was done with the posterior pericardial stitch of the "notouch' technique and the use of the Octopus Evolution Tissue Stabilizer TS 2000 and Starfish 2 Heart Positioner (Medtronic Inc., Minneapolis, MN, USA). The left internal mammary artery was always attached to the left anterior descending coronary artery and venous grafts were used for all other necessary grafts. A cell saver was used in all patients. Heat loss during surgery was prevented with a warm room temperature at $22{ }^{\circ} \mathrm{C}$, forced air warming blankets (Bair Hugger Blanket Model 630 cardiac and Bair Hugger Heater/Cooler Model 505 at $43{ }^{\circ} \mathrm{C}$, Augustine Medical Inc., Eden, Prairie, MN, USA), warmed fluids (Ranger Blood/Fluid Warming System at $41{ }^{\circ} \mathrm{C}$, Arizant Health Care Inc., Eden, Prairie, MN, USA), and rebreathing anaesthesia circuits. Preload was optimised during surgery with gelofusin and the Trendelenburg position. Mean arterial pressure was maintained above $70 \mathrm{mmHg}$ to allow a safety margin above the critical coronary perfusion pressure. Therefore, atrial pacing and small doses of $50 \mu \mathrm{g}$ of phenylephrine were used, when indicated. Cardiovascular performance was monitored with the continuous mixed venous oxygen saturation and the consequences of myocardial ischaemia were monitored with the diastolic pulmonary artery pressure.

Postoperative intensive care management was standardised. After four hours of positive pressure ventilation with $8 \mathrm{cmH}_{2} \mathrm{O}$ of positive end expiratory pressure (PEEP), and haemodynamic and ventilatory parameters being stable, the sedation was stopped and patients were rapidly weaned from the ventilator. A standardised lung protective ventilatory strategy is routinely applied in our hospital, and all measures were taken in the operating room and in the intensive care unit (ICU) to prevent postoperative atelectasis.

Blood and sputum samples for I1-8 and TNF- $\alpha$ were taken at four time points. After induction of anaesthesia (T1), at the end of surgery (T2), two hours after arrival in the ICU (T3) and four hours after arrival in the ICU (T4). The blood samples were collected in evacuated blood collecting tubes $(10 \mathrm{~mL}$, Monojet, Sherwood Medical, Ballymoney, N. Ireland) containing ethylenediamine-teraacetic acid. The sputum samples were taken by inserting a sterile suction catheter into the endotracheal tube and advancing it until it wedged. Sterile saline, $0.5 \mathrm{ml} . \mathrm{kg}^{-1}$, was injected and immediately aspirated into a sputum trap. ${ }^{9}$ Blood and sputum samples 
were centrifuged (1500 rpm, $10 \mathrm{~min}$, room temperature) and plasma and sputum was stored at $-70^{\circ} \mathrm{C}$ until analysis

Concentrations of IL- 8 and TNF- $\alpha$ were analysed in the commercially available PeliKine Compact ELISA system (Sanquin, Amsterdam, The Netherlands). Serum samples were diluted twofold for both cytokines, whereas sputum samples were diluted tenfold for IL- 8 and twofold for TNF- $\alpha$. . Consecutive samples, either serum or sputum, of the same patient were run on the same ELISA plate in duplicate. Results were only accepted if duplicates had coefficient of variation values less than $20 \%$, unless the calculated concentrations were below the lowest standard. Samples with higher coefficient of variation values were re-analysed. Concentrations were calculated from a 7-point standard curve ranging from 1-240 pg. $\mathrm{ml}^{-1}$ for IL-8 and from 1.4-1000 pg.ml ${ }^{-1}$ for TNF- $\alpha$, taking into account the respective dilution factors. The correlation coefficient for the curve fit of the standard curve was always $>99.5 \%$.

The oxygenation ratios $\left(\mathrm{PaO}_{2} / \mathrm{F}_{\mathrm{I}} \mathrm{O}_{2}\right)$ and the amount of extravascular lung water (EVLWI; PICCO system, Pulsion Medical Systems, Munich, Germany) were measured at the four time points.

The primary outcome variable was the oxygenation ratio, secondary outcome variables were the EVLWIs and the inflammatory markers in blood and BAL. A sample size calculation estimated that 19 patients would be required in each group to detect a $15 \%$ difference in the $\mathrm{PaO}_{2} / \mathrm{F}_{\mathrm{I}} \mathrm{O}_{2}$ ratio between groups, with $\alpha=0.05$ and $80 \%$ power.

Investigators analysing the laboratory data and the oxygenation ratio and EVLWIs were blinded for the study groups. Clinical characteristics were analysed using student t-tests. Generalized Linear Models (GLM) with repeated measures analysis of variance was used to compare the two groups. Analyses were performed with group (two levels: CBP group and off pump group) as between-subject variable and time (four levels: T1, T2, T3 and T4) as the within-subject factor. Where significant differences were found, independent sample t-test analyses were performed for differences between the two groups and paired $t$-test analyses for differences within group, comparing the differences between pre-test and followup time points. Differences were considered significant at p-values less than 0.05 . All data are presented as mean (SD). All statistical analyses were performed using SPSS 11.5 .

\section{RESULTS}

The clinical characteristics of the study population are shown in the table. No myocardial infarctions, re-sternotomies or other major complications were observed. There were fewer grafts in the off-pump group, where surgery time was also shorter. (Table 1) 
Table 1. Clinical characteristics of the study population.

\begin{tabular}{lccccc}
\hline & \multicolumn{2}{c}{ CPB } & \multicolumn{2}{c}{ Off-pump } & P value \\
\hline Age (years) & 61.4 & $(9.5)$ & 63.7 & $(12.8)$ & 0.525 \\
Height (cm) & 175 & $(7.9)$ & 173 & $(9.7)$ & 0.443 \\
Weight (kg) & 86.5 & $(13.2)$ & 80.5 & $(12.6)$ & 0.175 \\
Sex (M : F) & $16: 4$ & & & $14: 6$ & 0.152 \\
Body Mass Index (kg.m-2) & 27.9 & $(3.5)$ & 26.9 & $(2.8)$ & 0.330 \\
Number of grafts (n) & $3.9^{\star}$ & $(1.2)$ & 2.1 & $(1.1)$ & 0.000 \\
Aortic cross-clamp (min) & 53.7 & $(20.9)$ & & & \\
CPB time (min) & 81.3 & $(26.9)$ & & & \\
Surgery time (min) & $162^{+}$ & $(24)$ & 113 & $(33)$ & 0.000 \\
Intubation time in ICU (h) & 7.5 & $(3.8)$ & 7.8 & $(3.3)$ & 0.832 \\
ICU stay (h) & 29.0 & $(13.8)$ & 26.0 & $(17.1)$ & 0.569 \\
Hospital Length-of-Stay (day)s & 6.3 & $(1.9)$ & 6.3 & $(1.7)$ & 0.973 \\
Packed Cells (n units) & 1.3 & $(2.3)$ & 1.6 & $(2.1)$ & 0.722 \\
Fresh Frozen Plasma (n units) & 0.0 & $(0.7)$ & 0.0 & $(0.0)$ & 0.324 \\
Platelets (n units) & 0.1 & $(0.1)$ & 0.0 & $(0.0)$ & 0.324 \\
\hline
\end{tabular}

Values are mean $(\mathrm{SD}) . \mathrm{CPB}=$ group who received cardiopulmonary bypass; * $[\mathrm{CI} 95 \% 1.6-2.5$ and 3.4 $-4.5] ;{ }^{+}$[CI $95 \% 97-128$ and $\left.149-175\right]$

In figure 1, the EVLWI and oxygenation ratios are shown. EVLWI measurements at baseline were the same in both groups. No increases from baseline nor any differences between the two groups were observed in the present study at any timepoint; $(\mathrm{CPB}$, mean $(\mathrm{SD}): \mathrm{T} 1=6.3(3.6), \mathrm{T} 2=5.8(1.9), \mathrm{T} 3=6.4(1.8), \mathrm{T} 4=6.0(1.7)$ ml.kg ${ }^{-1}$; off-pump, mean (SD): $\mathrm{T} 1=5.6(1.2), \mathrm{T} 2=5.9(1.1), \mathrm{T} 3=6.1(1.3), \mathrm{T} 4=$ $\left.6.2(1.4) \mathrm{ml} \mathrm{kg}^{-1}\right)$. The oxygenation ratio $\left(\mathrm{PaO}_{2} / \mathrm{F}_{\mathrm{I}} \mathrm{O}_{2}\right)$ was the same at baseline in the CPB group and off-pump group. There were no increases from baseline and there were no significant differences between the two groups at the different measured time points.

In figure 2, the blood and bronchial levels of TNF- $\alpha$ and IL-8 are shown. Blood TNF- $\alpha$ baseline concentrations were the same in the two groups. TNF- $\alpha$ concentrations in the $\mathrm{CPB}$ group increased significantly at timepoint 2 compared to baseline $(p=0.004)$ and were at that timepoint also significantly higher than in the off-pump group: mean (SD) $=3.68(2.5)$ vs $2.20\left(1.2\right.$ pg. $\left.\mathrm{ml}^{-1}\right), \mathrm{p}=0.043[\mathrm{CI}$ $0.05-2.9]$. TNF- $\alpha$ concentrations in the off-pump group remained around their baseline level at all the measured timepoints. Baseline blood IL-8 concentrations were the same in both groups. In both groups, IL-8 concentrations increased significantly from baseline concentrations at all timepoints. At timepoints $\mathrm{T} 2$ and T3, the IL-8 concentrations in the CPB group were significant higher than in the off-pump group: mean $(\mathrm{SD})=19.45(10.8)$ vs $6.31(5.3) \mathrm{pg} \cdot \mathrm{ml}^{-1}(\mathrm{P}=0.001)[\mathrm{CI}$ 6.9-19.3] and $18.86(10.9)$ vs $8.44(6.5) \mathrm{pg} \cdot \mathrm{ml}^{-1}(\mathrm{P}=0.001)$ [CI 3.7-17], respectively. 


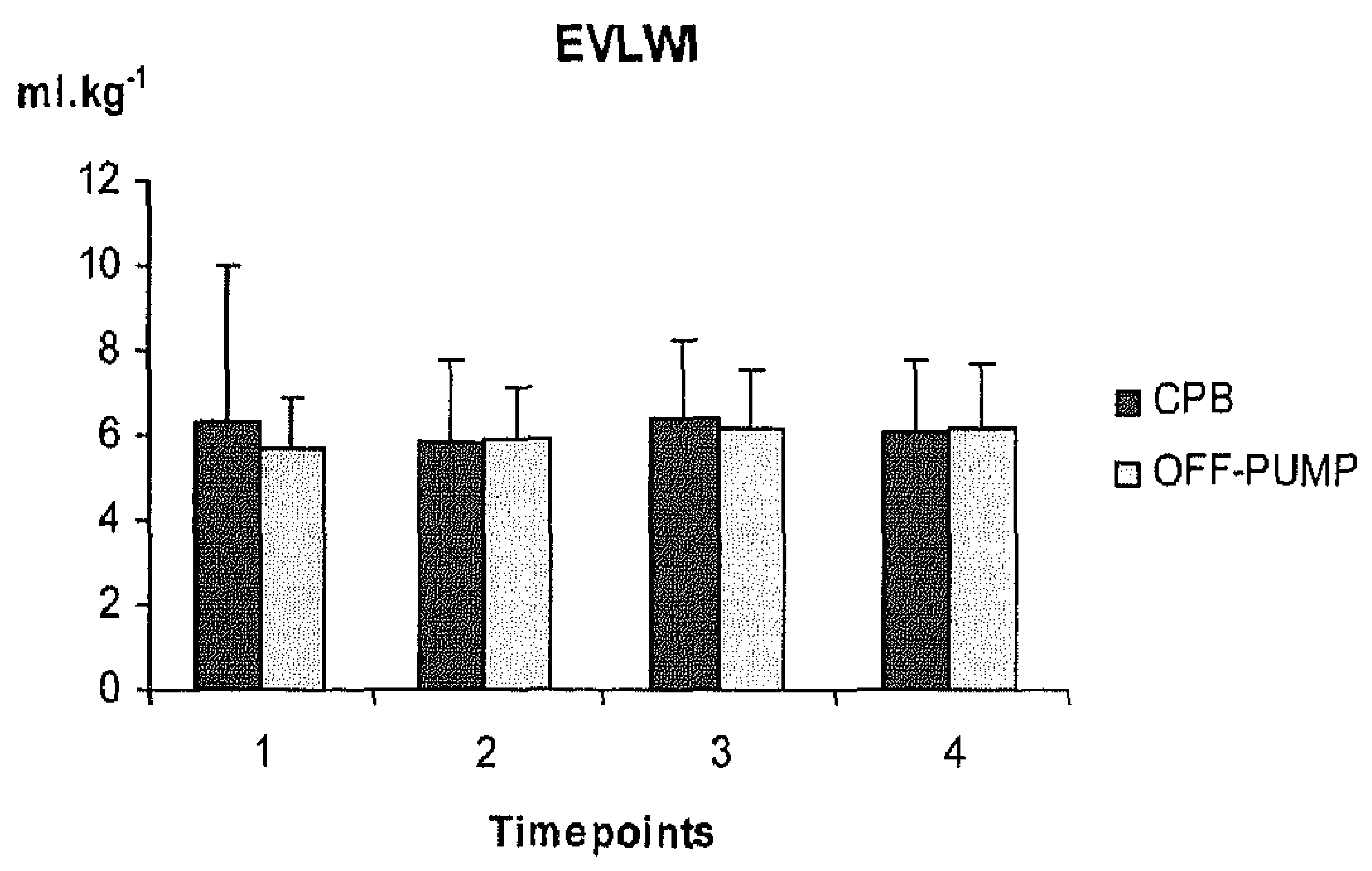

$\mathrm{PaO}_{2} / \mathrm{FiO}_{2}$ ratio

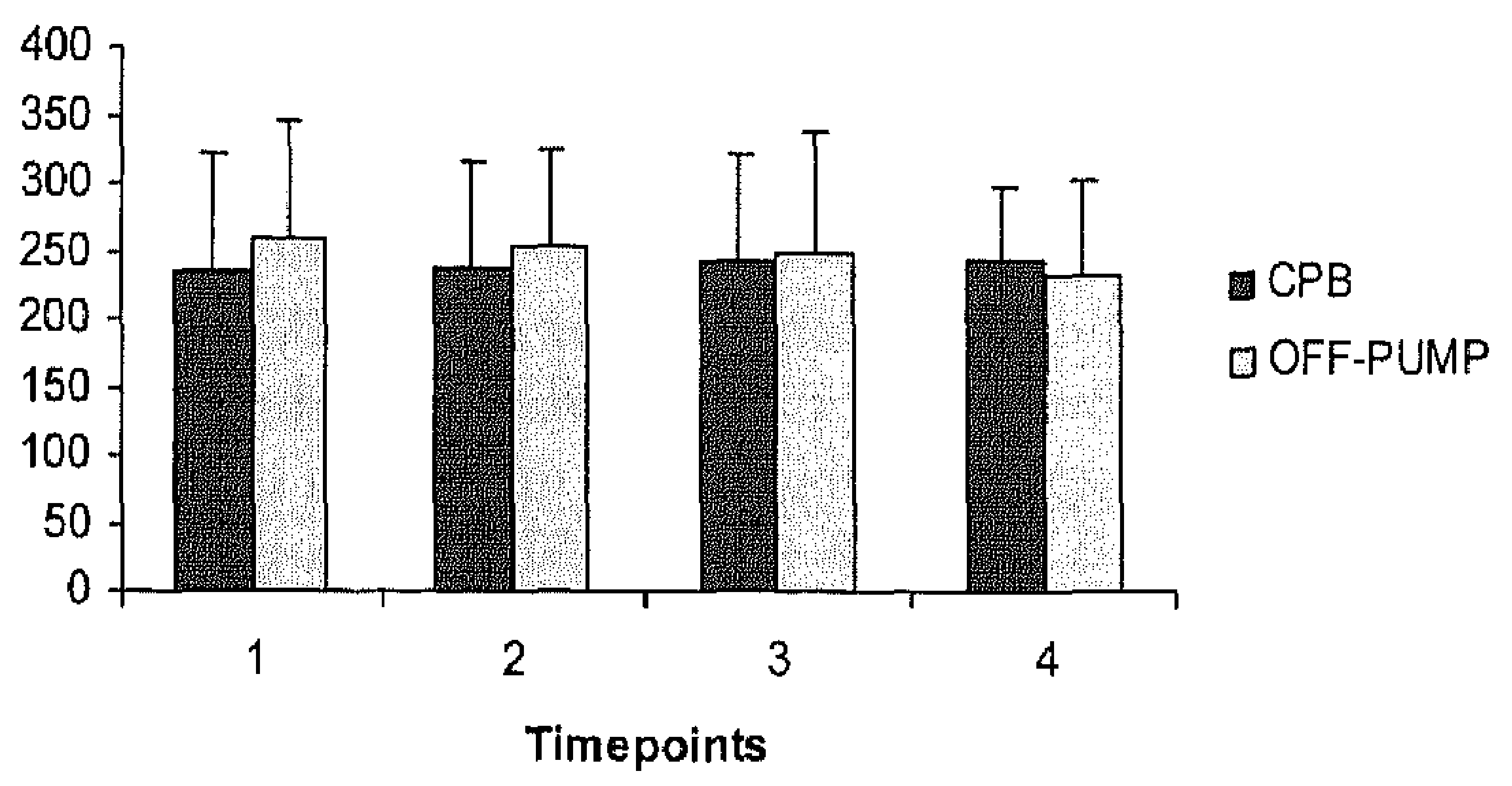

Figure 1. Extra Vascular Lung Water Index and Oxygenation ratios in the two study groups.

Data are given as mean (SD); Timepoints: $1=$ after induction of anaesthesia, $2=$ at the end of surgery, $3=$ two hours after arrival in the ICU, $4=$ four hours after arrival in the ICU; Off-pump = off-pump coronary artery surgery; $\mathrm{CPB}=$ conventional coronary artery surgery.

Bronchial baseline concentrations of TNF- $\alpha$ were the same in both groups. At timepoints $\mathrm{T} 2$ and $\mathrm{T} 3$ in the $\mathrm{CPB}$ group $(\mathrm{P}=0.043$ and $\mathrm{P}=0.017)$ and at timepoint $\mathrm{T} 3$ in the off-pump group $(\mathrm{P}=0.023)$, the TNF- $\alpha$ concentrations were significantly increased compared to baseline. There were no differences between the two groups at any measured timepoint. Bronchial IL-8 concentrations were the same at baseline in the two groups. In both groups, bronchial IL-8 concentrations increased significantly from baseline at all time points. There were no significant differences in bronchial IL-8 concentrations between the two groups. 

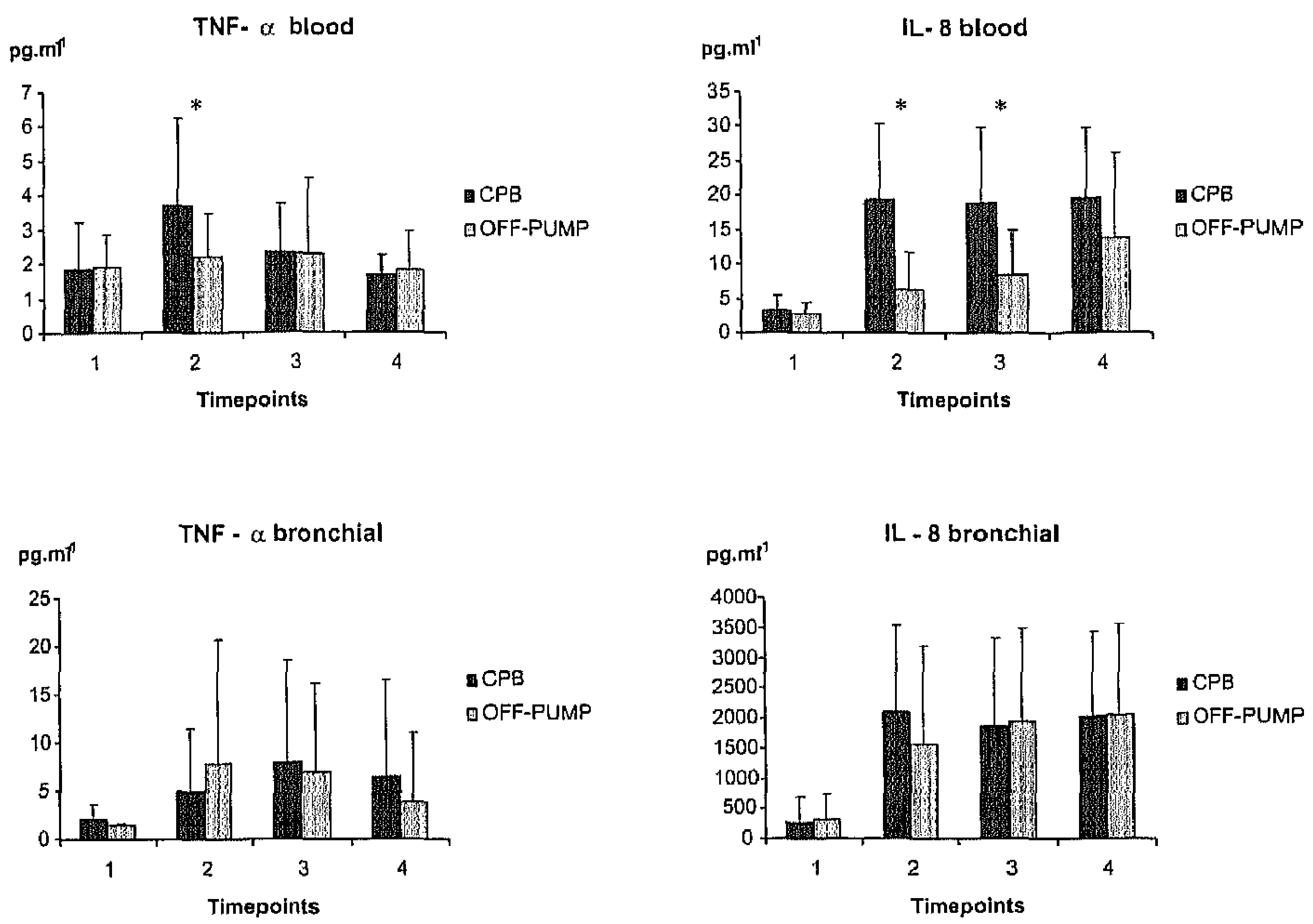

Figure 2. Concentrations of TNF- $\alpha$ and IL-8 in blood and in bronchoalveolar lavage fluid (BAL). Data are given as mean (SD) . $\star=P<0.005$, significant difference between the two groups; Timepoints: $1=$ after induction of anaesthesia, $2=$ at the end of surgery, $3=$ two hours after arrival in the ICU, $4=$ four hours after arrival in the ICU; Off-pump = off-pump coronary artery surgery; CPB = conventional coronary artery surgery.

\section{DISCUSSION}

The hypothesis underlying the present study was that off pump surgery is association with a reduced pulmonary inflammatory response compared to surgery requiring cardiopulmonary bypass, and this will be reflected in better postoperative lung function. However, no differences in postoperative lung function were found between the CPB and off-pump groups, as evidenced by the oxygenation ratios and LVSWIs. In addition no differences were observed between the pro-inflammatory cytokines TNF- $\alpha$ and IL-8 in the BAL of the two groups. We found that CPB was associated with more systemic inflammation than off-pump surgery, as shown by the blood levels of TNF- $\alpha$ and IL- 8 .

Cardiopulmonary bypass is associated with an intense inflammatory response because of the change from pulsatile to continuous flow, blood contact with the artificial bypass surface, cold cardiac ischaemia, and hypothermia. This inflammatory response causes temporary dysfunction of nearly every organ. ${ }^{10}$ Postoperative lung dysfunction may consist of increased pulmonary capillary protein permeability, increased extravascular lung water or oedema and atelectasis. These compli- 
cations often lead to hypoxaemia, ventilator dependency and adverse outcome. ${ }^{11}$ Evidence from a number of randomised controlled studies shows that the inflammatory response produced by CPB surgery is much more severe than that produced off-pump surgery. ${ }^{12-15}$ Avoidance of inflammation due to CPB is a major reason for the recent resurgence of interest in off-pump surgery. In our study, the blood levels of TNF- $\alpha$ and IL-8, as markers of systemic inflammation, indicate that the systemic inflammatory response was more pronounced in the $\mathrm{CPB}$ group than in the off-pump group. However, comparison of the inflammatory effects of $\mathrm{CPB}$ between different studies and/or institutions is extremely difficult, because the magnitude of the inflammatory response is largely affected by the CPB-management. Indeed, biocompatible materials, filters, pulsatility of flow, prime, cardiotomy suction and use of anti-inflammatory medications are all factors that affect the severity of the inflammatory response or its consequences. ${ }^{16-19}$ In our institution, we routinely use biocomapible materials, filters, pulsatile flow and restrict cardiotomy suction. The prime consists of a colloid solution of gelatin and albumin; whenever the colloidal osmotic pressure is below $2 \mathrm{kPA}$, additional albumin is added to the circuit. We do not use steroids or aprotinin. This 'anti-inflammatory' CPB-management could explain why the magnitude of the inflammatory response in the CPB group, and consequently, the difference in inflammation between the $\mathrm{CPB}$ group and the off-pump group, is less in the present study compared to some other studies.

No differences were observed in the amount of TNF- $\alpha$ and IL- 8 in the BAL between the two study groups. This could suggest that the difference in systemic inflammation between the two groups is too small to cause any difference in inflammation at the organ level. However, interpretation of the results of organ inflammation should be made very cautiously, because of the limited experience with these data. Frass and collegues found that CPB caused impairment of the antioxidant defense system as well as a protease/antiprotease imbalance in the blood and broncheoalveolar lavage fluid. ${ }^{20}$ Massoudy and colleagues described increased pulmonary cytokine concentrations in the pulmonary vein after $\mathrm{CPB}$ surgery as evidence for inflammatory responses of the lung ${ }^{11}$ TNF- $\alpha$ and IL- 8 are cytokines found in the alveoli of the lung and detection of IL- 8 in bronchial secretions is associated with the presence of nosocomial pneumonia ${ }^{21}$ or acute lung injury. ${ }^{9}$ In BAL of susceptible patients, increased concentrations of IL-8 are associated with ARDS. ${ }^{22}$

The inflammatory response to injury depends on the balance of pro and antiinflamattory mediators as well as the absolute values of certain compounds. We only measured the cytokine interleukin- 8 and tumor necrosis factor because they are both early markers of lung injury. ${ }^{11,21,23}$ and very often used as representative samples of systemic inflammation. ${ }^{15}$

The major reason for studying off-pump surgery patients was the expectation that less inflammation would reduce lung injury. Our assumption that the differ- 
ence was largely due to $\mathrm{CPB}$ was consistent with the hypothesis that the general inflammatory response associated with $\mathrm{CPB}$ allows macromolecules to enter the pulmonary interstitium and the alveoli contributing to abnormalities of pulmonary gas exchange and to pulmonary edema."

The $\mathrm{PaO}_{2} / \mathrm{F}_{\mathrm{I}} \mathrm{O}_{2}$ ratio is a well recognised indicator of lung dysfunction ${ }^{2+}$ and, in combination with other lung-related parameters, is used in the definitions of ALI and ARDS. ${ }^{25}$ The accumulation of extravascular lung water due to capillary leakage has been considered an important pathophysiologic mechanism of impaired lung function after cardiac surgery and correlates well with the severity of lung injury. ${ }^{26}$ Therefore, we measured the EVLWI with the PiCCO system, which is a valid and reliable instrument for measuring EVLW compared to the golden standard, gravimetric measurement. ${ }^{27,28}$ EVLWI was found to be slightly increased after cardiac surgery with $\mathrm{CPB}$, being higher in non-pulsatile vs pulsatile $\mathrm{CPB},{ }^{\text {th }}$ when a crystalloid prime was used versus a colloid prime ${ }^{29}$ and when the colloid osmotic pressure during CPB is maintained at normal levels. ${ }^{17}$ It is lower when steroids are given ${ }^{18}$ indicating the sensitivity of this parameter. Some previous studies have failed to show any beneficial effect on pulmonary gas exchange of offpump techniques. ${ }^{4-8}$ The present investigation confirms and extends insights from previous work. Our study is the first investigation to study pulmonary outcome in CPB surgery versus off-pump surgery by measuring not only the oxygenation ratios but also extravascular lung water, linking the pulmonary outcome measures with systemic inflammation and pulmonary inflammation. In the present study, no differences in the oxygenation ratios and EVLWI were observed between the two groups. Moreover, no changes from baseline were observed concerning these two parameters. Indeed, the activated pulmonary cytokine response did not result in an increase in EVLW. EVLWI measurements remained at mean baseline values between 5.85-6.4 ml. kg-1 in the CPB group and between $5.64-6.2 \mathrm{ml}^{-1} \mathrm{~kg}^{-1}$ in the off-pump group. These data are below $7 \mathrm{ml} . \mathrm{kg}^{-1}$, the normal range for EVLWI, and well below the mean (SD) values 12.3 (3.4) $\mathrm{ml}^{\mathrm{kg}} \mathrm{kg}^{-1}$ as seen in patients with ARDS. ${ }^{301}$ Thus, our results indicate that our CPB current technique for up to 90 minutes has little effect on postoperative respiratory dysfunction compared with that reported over the last two decades.

The major potential limitation of this study lies in the design weakness of nonrandomization. However, the off-pump patients were exclusively defined by the absence of disease in the circumflex territory and otherwise met all the criteria to enter the study. As less than $20 \%$ of our patients were considered suitable for offpump surgery at that time, it was impractical to randomize these patients into a CPB group and an off-pump group. Furthermore, the study was performed in relatively healthy patients and these results may not extrapolate to older and sicker patients. Systemic inflammation increases with duration of surgery. In the present study, surgery time was shorter in the off-pump group. To what extend this differ- 
ence in surgery time might have affected our measured levels of pro-inflammatory markers, remains speculative.

Adverse effects of anaesthesia, surgery, and ICU management on lung function are numerous and have been the subject of several previous investigations. ${ }^{31,32}$ Surgical factors include pleural opening, mechanical alteration in rib cage and sternal alignment, phrenic nerve dysfunction, reflex-mediated decreases in diaphragmatic function, direct contact with the lungs, cardiac manipulation with subsequent pulmonary oedema, and inflammation due to surgical trauma. Anaesthesia and ICU management factors include the use of potent vasodilators with subsequent shunting, moderate positive end-expiratory pressure during $\mathrm{CPB}$, positive pressure ventilation, restriction on coughing and deep breathing by postoperative pain and recruitment maneuvers. Although some of these may result in increased interstitial fluid in the lung, the most consistent result of all these processes is regional atelectasis. ${ }^{33,34}$ Therefore, if pulmonary gas exchange deteriorates after cardiac surgery, it may be a consequence of the anaesthetic, surgical and ICU techniques used or of the degree of inflammation.

In the present study, all possible measures were taken to decrease the inflammatory effects of $\mathrm{CPB}$ and to prevent postoperative atelectasis, and no significant postoperative lung injury was observed in our patients. The importance of these two strategies is unclear but the lung injury previously observed in similar studies may be related to shortcomings in delivering these strategies. ${ }^{11,31,32}$ The optimal strategy to attenuate the inflammatory response to $\mathrm{CPB}$ and to prevent and treat regional atelectasis remains to be defined. 


\section{REFERENCES}

1. Taggart DP, El-Fiky M, Carter R, Bowman A, Wheatley DJ. Respiratory dysfunction after uncomplicated cardiopulmonary bypass. Annals of Thoracic Surgery 1993; 56: 1123-8.

2. Kirklin JK, Westaby S, Blackstone EH, Kirklin JW, Chenoweth DE, Pacifico AD. Complement and the damaging effects of cardiopulmonary bypass. Journal of Thoracic and Cardiovasular Surgery $1983 ; 86: 845-75$.

3. Royston D, Minty BD, Higenbottam TW, Wallwork J, Jones GJ. The effect of surgery with cardiopulmonary bypass on alveolar-capillary barrier function in human beings. Annals of Thoracic Surgery 1985; 40: 139-41.

4. Kochamba GS, Yun KL, Pfeffer TA, Sintek CF, and Khonsari S. Pulmonary abnormalities after coronary arterial bypass grafting operation: cardiopulmonary bypass versus mechanical stabilization. Annals of Thoracic Surgery 2000;69: 1466-70.

5. Cox CM, Ascione R, Cohen AM, Davies IM, Ryder IG, and Angelini GD. Effect of cardiopulmonary bypass on pulmonary gas exchange: a prospective randomised study. Annals of Thoracic Surgery 2000; 69: 140 - 5.

6. Taggart DP. Respiratory dysfunction after cardiac surgery: effects of avoiding cardiopulmonary bypass and the use of bilateral internal mammary arteries. European Journal of Cardiothoracic Surgery 2000; 18:31-7.

7. Montes FR, Maldonado JD, Paez S, Ariza F. Off-Pump versus on-pump coronary artery bypass surgery and postoperative pulmonary dysfunction. Journal of Cardiothoracic and Vascular Anesthesia 2004; 18: 698-703.

8. Syed A, Fawzy $H$, Farag $A$ and Nemlander A. Comparison of pulmonary gas exchange in OPCAB versus conventional CABG. Heart, Lung and Circulation 2004; 13:168-72.

9. Krupa A, Kato H, Matthay MA, and Kurdowska A. Proinflammatory Activity of anti-IL-8 autoantibody: IL -8 complexes in alveolar edema fluid from patients with acute lung injury. American Journal of Physiology - Lung Cellular and Molecular Physiology 2004; 286: L1105L13.

10. Butler J, Rocker GM, Westaby S. Inflammatory response to cardiopulmonary bypass. Annals of Thoracic Surg 1993; 55: 552-9.

11. Massoudy P, Zahler S, Becker BF, Braun S L, Barankay A, and Meisner H. Evidence for inflammatory responses of the lungs during coronary artery bypass grafting with cardiopulmonary bypass. Chest 2001;119:31- 6 .

12. Ascione R, Lloyd CT, Underwood M J, Lotto A A, Pitsis AA, and Angelini GD. Inflammatory response after coronary revascularization with or without cardiopulmonary bypass. Annals of Thoracic Surgery 2000; 69: 1198-240.

13. Wan S, Izzat MB, Lee TW, Wan IY, Tang NLS and Yim AP. Avoiding cardiopulmonary bypass in multivessel CABG reduces cytokine response and myocardial injury. Annals of Thoracic Surgery $1999 ; 68: 52-7$.

14. Diegeler $A$, Doll $\mathrm{N}, \mathrm{R}$ auch $\mathrm{T}$ et al. Humoral immune response during coronary artery bypass grafting: a comparison of limited approach, "off-pump" technique, and conventional cardiopulmonary bypass. Circulation 2000, 102: III95-III100.

15. Matata BM, Sosnowski AW, Galinanes M. Off-pump bypass graft operation significantly reduces oxidative stress and inflammation. Annals of Thoracic Surgery 2000; 69:785-91.

16. Boldt J, Zickmann B, Dapper F and Hempelmann G. Does the technique of cardiopulmonary bypass affect lung water content? European Journal of Cardiothoracic Surgery 1991;5:22 - 6 .

17. Eising GP, Niemeyer M, Gunther Th et al. Does a hyperoncotic cardiopulmonary bypass prime affect extravascular lung water and cardiopulmonary function in patients undergoing artery bypass surgery? European Journal of Cardiothoracic Surgery 2001;20:282-9. 
18. Von Spiegel T, Giannaris S, Wietasch GJ et al. Effects of dexamethasone on the intravascular and extravascular fluid balance in patients undergoing coronary bypass surgery with cardiopulmonary bypass. Anesthesiology 2002;96:82-34.

19. Shimamoto A, Kanemitsu S, Fujinaga $\mathrm{K}$ et al. Biocompatibility of silicone-coated oxygenator in cardiopulmonary bypass. Annals of Thoracic Surgery 2000; 69: 115-20.

20. Frass $O \mathrm{M}$, Buhling $\mathrm{F}$, Trager $\mathrm{M}$ et al. Antioxidant and antiprotease status in peripheral blood and BAL fluid after cardiopulmonary bypass. Chest 2001; 120: 1599-1608.

21. Rodriguez JL, Miller CG, DeForge LE et al. Local production of interleukine-8 is associated with nosoconial pneumonia. Journal of Trauma 1992;33: 74-81.

22. Donnelly SC, Strieter RM, Kunkel SL et al. Interleukin-8 development of adult respiratory distress syndrome in at-risk patient groups. The Lancet 1993; 341:643-7.

23. Ware LB, Matthay MA. The acute respiratory distress syndrome. New England Journal of Medicine $2000 ; 342: 1334-49$.

24. Gould MK, Ruoss SJ, Rizk NW et al. Indices of hypoxemia in patients with acute respiratory distress syndrome: reliability, validity, and clinical usefulness. Critical Care Medicine 1997;25;6-8.

25. Wyncoll DL and Evans TW. Acute Respiratory Distress Syndrome. The Lancet 1999; 354: 497 -501 .

26. Kuzkov VV, Kirov MY, Sovershaev MA et al. Extravascular lung water determined with single transpulmonary thermodilution correlates with the severity of sepsis-induced acute lung injury. Critical Care Medicine 2006; 34: 1-7.

27. Katzenelson $\mathrm{R}$, Perel A, Berkenstadt $\mathrm{H}$ et al. Accuracy of transpulmonary thermodilution versus gravimetric measurement of extravascular lung water. Critical Care Medicine 2004;32, 1550-4.

28. Kirov M, Kuzkov VK, Kuklin VN, Waerhaug K, Bjertnaes LJ. Extravascular lung water assessed by transpulmonary single thermodilution and postmortem gravimetry in sheep. Critical Care 2004; 8: R451-R8.

29. Boldt J, van Bormann B, Kling D, Scheld HH, Hempelmann G. The influence of extracorporeal circulation on extravascular lung water in coronary surgery patients. Journal of Cardiothoracic and Vascular Surgery 1986;345: 110-5.

30. Patroniti N, Bellani G, Maggioni E, Manfio A, Marcora, Pesenti A. Measurement of pulmonary edema in patients with acute respiratory distress syndrome. Critical Care Medicine 2005; 33: 2547-54.

31. Johnson $D$, Hurst $T$, Thomson $D$ et al. Respiratory function after cardiac surgery. Journal of Cardiothoracic and Vascular Anesthesia 1996; 10:571-7.

32. Vargas FS, Terra-Filho M, Hueb W, Teixeira LR, Cukier A, Light RW. Pulmonary function after coronary artery bypass surgery. Respiratory Medicine 1997; 91:629-33.

33. Tenling A, Hachenberg $T$, Tyden $H$, Wegenius $G$, Hedenstierna $G$. Atelectasis and gas exchange after cardiac surgery. Anesthesiology 1998; 89:371-8.34.Magnusson L, Zemgulis V, Tenling A et al. Use of a vital capacity maneuver to prevent atelectasis after cardiopulmonary bypass. Anesthesiology 1998; 88: 134-42. 


\section{CHAPTER 8}

\section{Assessment of cardiac responsiveness to fluid therapy after cardiac surgery}

John H. Heijmans ${ }^{1}$, Yuri M. Ganushak ${ }^{2}$, Maurice S. Theunissen ${ }^{1}$, Jos G. Maessen ${ }^{2}$ and Paul M.H.J. Roekaerts ${ }^{3}$

${ }^{1}$ Department of Anesthesiology. ${ }^{2}$ Department of Cardiothoracic surgery.

${ }^{3}$ Department of Intensive Care and Anesthesiology

Accepted for publication: Journal of Cardiothoracic and Vascular Surgery 


\section{ABSTRACT}

Objectives. Central venous pressure (CVP) and pulmonary capillary wedge pressure (PCWP) are insensitive preload markers and sometimes misleading. The introduction of the pulse contour method for monitoring of continuous cardiac output enabled the real-time quantification of stroke volume variation (SVV). Studies evaluating the accuracy of this parameter as a measure of preload are still limited and conflicting results have been published in cardiac surgical patients. The aim of this study was to reevaluate the predictive value of SVV regarding cardiac responsiveness to fluid therapy and to compare it with the standard preload variables in a clinical setting in the ICU after cardiac surgery.

Methods. The assessment of cardiac responsiveness to fluid therapy (HAES- steril $6 \% 10 \mathrm{~mL}$ * Body Mass Index) was performed in 92 coronary artery surgical patients after arrival in the ICU. After the fluid load, detailed hemodynamic measurements were performed. A 'responder' was defined as a patient with a gain in stroke volume index (SVI) of $5 \%$ or more from baseline value to the volume challenge.

Results. There were 47 responders to the fluid challenge and 45 nonresponders. Haemodynamic data before the fluid therapy show that stroke volume variation in the responders group was significantly higher than in the nonresponders groups $(9.7 \pm 4.3 \%$ versus $7.6 \pm 3.0 \%)$. The receiver operating characteristic curves for the baseline values of CVP, PCWP and SVV were constructed. The area under the curve for baseline values of SVV was significantly higher than random guess (area $=0.65, \mathrm{p}<0.05$ ), indicative of the value of SVV as a predictor of cardiac responsiveness to fluid therapy. The static preload parameters CVP and PCWP had no predictive value.

Conclusion. SVV is a good preload parameter for predicting and for on-line monitoring of cardiac responsiveness to fluid therapy. This functional preload marker is far superior to the static parameters CVP and PCWP.

\section{INTRODUCTION}

The initial postoperative course after cardiac surgery is characterised by impaired ventricular contractility and substantial changes in intravascular volume. The maintenance of an adequate preload during this period is crucial to optimise left ventricular performance and global oxygen delivery and may help in avoiding detrimental volume overload or inappropriate use of inotropic drugs.

Thus, in hypotensive patients, methods to unmask decreased preload and to predict whether cardiac output will increase or not with fluid therapy have been sought after for many years. Frequently used static preload indexes such as central venous pressure or pulmonary capillary wedge pressure often fail to provide reliable information on cardiac preload and are not capable of predicting a cardiac response 
to fluid therapy.' Measuring left ventricular end-diastolic area index by transesophageal echocardiography ${ }^{2}$ or assessment of intrathoracic blood volume index should provide an accurate estimation of hypovolemia but cannot be used routinely in most patients. ${ }^{3}$

Recently, dynamic preload variables, such as systolic pressure variation (SPV), pulse pressure variation (PPV) and stroke volume variation (SVV) have been introduced into clinical practice and were found to be superior to the standard static indicators of preload. ${ }^{4-13}$ All these new parameters are based on ventilation-induced changes in left ventricular stroke volume. ${ }^{14}$ Positive pressure ventilation is known to affect cardiovascular function due to intermittent increases in the intrathoracic pressure. The increase in intrathoracic pressure affects the preload and afterload of both ventricles, though its most common effect is to decrease systemic venous return and hence the cardiac output. Such a decrease is dependent on the intravascular volume status and is greater under hypovolemic conditions. Both SPV and PPV have been shown to permit titration of fluid therapy in various patient populations. However, SPV and PPV are more influenced by vasomotor tone than SVV, which is therefore considered the most accurate parameter.

The introduction of the pulse contour method for the monitoriong of continuous cardiac output enabled the real-time quantification of SVV. Studies evaluating the accuracy of this parameter as a measure of preload are still limited and were performed in small patient groups using the PiCCO (plus) system (Pulsion Medical Systems, Munich, Germany). Conflicting results from these studies have been published regarding the clinical use of SVV. ${ }^{15-2()}$ Clearly, further studies are required to investigate the value of dynamic preload parameters as predictors of fluid responsiveness in larger patient groups and in different clinical settings. In the present study, we used the LiDCO pulse-contour cardiovascular monitor (LiDCO Ltd, London, UK) to continuously assess SVV.

The aim of this study was to reevaluate the predictive value of SVV regarding cardiac responsiveness to fluid therapy and to compare it with the standard preload variables in a clinical setting in the ICU after cardiac surgery.

\section{METHODS}

After approval of the hospital medical ethics committee and written informed consent, ninety-two patients scheduled for elective coronary artery surgery, were included in the study. Exclusion criteria included left ventricular ejection fraction of less than $40 \%$, recent myocardial infarction, preoperative inotropic or intra-aortic balloon pump support, dysrhythmias, emergency surgery and significant pulmonary, endocrine, metabolic or neurologic disease. All patients were on chronic betaadrenoreceptor blocking drugs. The distribution of other medications affecting cardiovascular variables (nitrates, calcium antagonists, ACE inhibitors) was the same for all patients. Patients were premedicated with midazolam together with their 
individual beta-adrenoreceptor blocking drug. Anaesthesia consisted of a standardised total intravenous anaesthesia technique with propofol and alfentanil. Monitoring included a radial artery catheter and a pulmonary artery catheter (Swan Ganz catheter, Edwards Lifesciences, Irvine, CA, USA) connected to a Vigilance monitor (Edwards Lifesciences, Irvine, CA, USA) for continuous cardiac output and mixed venous oxygen saturation measurements. Additionally, the PulseCO Haemodynamic Monitor (LiDCO Limited, London, UK) was used for haemodynamic measurements. The cardiac output for the PulseCO was calibrated using the pulmonary artery thermodilution measurement technique. The stroke volume variation (SVV) was calculated by a formula $\left[S V V=\left(\mathrm{Sv}_{\max }-\mathrm{SV}_{\text {min }}\right) / \mathrm{Sv}_{\text {mean }}\right]$ over a time period of 10 seconds (PulseCO Haemodynamic Monitor, user's manual version 3) and expressed in \%. According to our standard hospital practice, all patients were ventilated and received sedative-analgesic infusions of propofolpiritramide after arrival in the ICU for four hours; thereafter, sedative-analgesic infusions were stopped and patients rapidly weaned from the ventilator. Ventilator settings included tidal volumes of $6 \mathrm{~mL} \cdot \mathrm{kg}^{-1}$ and a PEEP - level of $8 \mathrm{~cm} \mathrm{H}_{2} \mathrm{O}$. All patients received a standard dose of $0.5 \mu \mathrm{g} \cdot \mathrm{kg}^{-1} \cdot \mathrm{min}^{-1}$ nitroglycerine intravenously.

The assessment of cardiac responsiveness to fluid therapy was performed within four hours after arrival in the ICU, when the following criteria were met: (1) patient were fully rewarmed, centrally as well as peripherally; (2) patients were haemodynamically stable; (3) there were no important dysrhythmias; (4) the level of sedation was constant; (5) the level of analgesia was constant; (6) the administration rate of fluid was constant and low; (7) the amount of ungoing blood loss was limited to less than $50 \mathrm{ml}$ per half hour; (8) if the administration of inotropic drugs was necessary, these drugs were given in low dose and at a constant infusion rate; (9) infusions of drugs affecting peripheral resistance were given in low dose and at a constant infusion rate; (10) the respiratory parameters were stable without spontaneous breathing efforts by the patients; (11) tidal volumes were constant; (12) the level of positive end-expiratory pressure was stable; and (13) the attending supervising staff anaesthesiologist-intensivist decided that fluid therapy was indicated.

The fluid challenge was performed by infusion of HAES-sterile 6\% (Fresenius Kabi, Bad Homburg, Germany) $10 \mathrm{~mL}$ * BMI (body mass index) over a period of 15 minutes. Haemodynamic measurements included: stroke volume variation (SVV); central venous pressure (CVP); pulmonary artery capillary wedge pressure (PCWP); systolic, mean and diastolic arterial pressure (SAP, MAP, DAP); heart rate $(\mathrm{HR})$; mixed venous oxygen saturation $\left(\mathrm{SVO}_{2}\right)$; cardiac index $(\mathrm{CI})$; systemic vascular resistance (SVR) and stroke volume (SV). Measurements were performed in triplicate before the fluid challenge at minutes $-3,-2$ and -1 and after the fluid challenge at minutes $+1,+2$ and +3 . The average of these measurements was taken as the pre-challenge value and the post-challenge value.

A positive cardiac response to the fluid therapy was defined as a gain in stroke volume index (SVI) of $5 \%$ or more from baseline value, where SVI $=\mathrm{SV} / \mathrm{BSA}$ and 
SVI $\%=$ SVI (baseline) - SVI (after the challenge) * $100 /$ SVI (baseline). Patients with a SVI gain $\geq 5 \%$ were called 'responders' and patients with a SVI gain $<5 \%$ 'non-responders'.

\section{Statistics}

The One-way ANOVA test was used to compare parameters between the groups and the differences between the pre-load and post-load conditions. Results are shown as mean \pm SD. A P value of $<0.05$ was considered a significant difference. Evaluation of the ability of the tested parameters to predict positive fluid responsiveness was performed by constructing receiver operating characteristic curves. The area under each curve was calculated, and the respective values were compared. A value of ROC curve of 1.0 indicates perfect performance with $100 \%$ sensitivity and $100 \%$ specificity for the corresponding indicator, whereas the value of 0.5 means that the predictive performance of the indicator is no better than chance.

\section{RESULTS}

There were 47 responders to the fluid challenge (Group 1) and 45 nonresponders (Group 2). There were no differences between the two groups concerning demographic and intraoperative data (Table 1).

Haemodynamic data before the fluid therapy are shown in table 2. SVV in the responders group was significantly higher than in the non-responders groups $(9.7 \pm$ $4.3 \%$ versus $7.6 \pm 3.0 \%$ ). CI and SVI were lower and the SVRI higher in the responders group.

In table 3 , the haemodynamic data of the responders are shown before and after the fluid therapy. The fluid therapy in this group was associated with increases in SVV from $7.5 \%$ to $9.7 \%(\mathrm{P}=0.01)$ and in CVP from $5.6 \%$ to $6.6 \%(\mathrm{P}=0.04)$. CI, SV and SVI increased also, and SVRI decreased from 2330 to 1983 dynesec. $\mathrm{cm}^{-5} \cdot \mathrm{m}^{-2}$.

In table 4 , the haemodynamic data of the non-reponders are shown before and after the fluid therapy. In this group, fluid therapy did not result in increases in cardiac performance. SVV was the only preload parameter which indicated that a fluid challenge had been given: SVV decreased from $7.6 \pm 3.0 \%$ to $6.1 \pm 2.6 \%$ (P $=$ $0.02)$.

The ROC curves for the baseline values of CVP, PCWP and SVV pressure are presented in figures 1,2 and 3, respectively. The area under the curve for baseline values of SVV was significantly higher than random guess (area $=0.65, \mathrm{p}<0.05$ ), indicative of the value of SVV as a predictor of cardiac responsiveness to fluid therapy. The static preload parameters CVP and PCWP had no predictive value. 

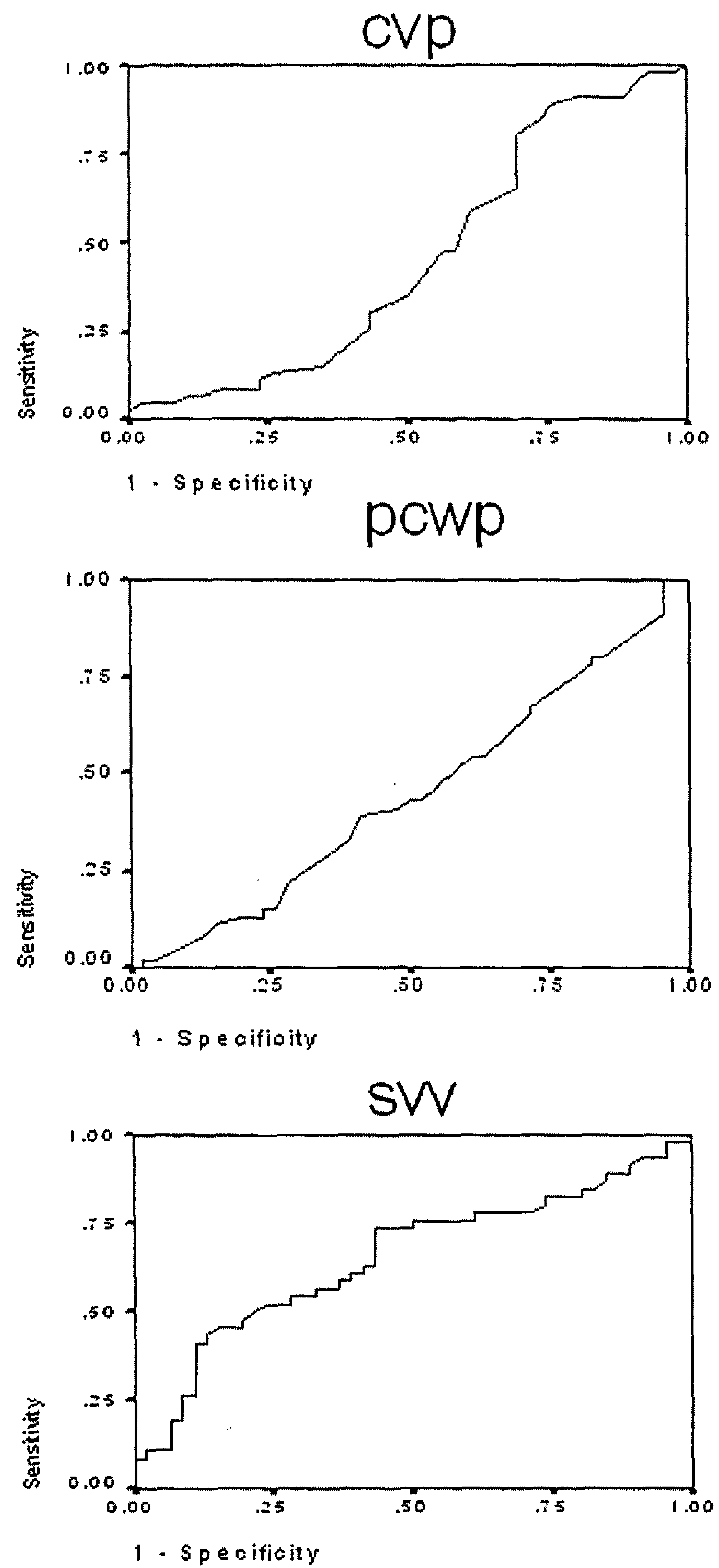

Figure 1,2 and 3 Receiver operating characteristic (ROC) analysis for the central venous pressure (CVP), the pulmonary capillary wedge pressure (PCWl') and the stroke volume variation (SVV). 
Table 1. Demographic data

\begin{tabular}{lccccc}
\hline & \multicolumn{2}{c}{$\begin{array}{c}\text { Group 1 } \\
(\mathrm{n}=47)\end{array}$} & \multicolumn{2}{c}{$\begin{array}{c}\text { Group 2 } \\
(\mathrm{n}=45)\end{array}$} & P value \\
& Mean & SD & Mean & SD & \\
\hline Age (years) & 64.6 & 9.9 & 64.1 & 9.5 & 0.815 \\
Heigth (cm) & 172.8 & 8.6 & 172.6 & 8.8 & 0.918 \\
Weight (kg) & 82.8 & 13.3 & 84.4 & 15.6 & 0.582 \\
BMI (kg m-2) & 27.6 & 3.9 & 27.8 & 3.5 & 0.862 \\
CPB time (min) & 63.2 & 19.4 & 73.0 & 21.0 & 0.046 * \\
Aorta X time (min) & 38.9 & 12.6 & 41.7 & 10.4 & 0.579 \\
Anastomoses (numbcr) & 3.3 & 1.2 & 3.6 & 1.2 & 0.152 \\
\hline
\end{tabular}

Abbreviations. BMI: body mass index; CPB: cardiopulmonary bypass; X: cross clamp. Group 1: responders (gain in stroke volume index of $5 \%$ or more from baseline value after fluid therapy; Group 2: nonresponders. Values are mean \pm standard deviation. ${ }^{\star} \mathrm{P}<0.05$ was considered a significant difference.

Table 2. Hemodynamic data before fluid therapy

\begin{tabular}{lccccc} 
& \multicolumn{2}{c}{ Group 1 } & \multicolumn{2}{c}{$\begin{array}{c}\text { Group 2 } \\
\text { (n=45) }\end{array}$} \\
& Mean & SD & Mean & SD \\
\hline SVV (\%) & 9.66 & 4.34 & 7.57 & 2.96 & $0.01^{\star}$ \\
CVP (mmHg) & 5.61 & 2.19 & 6.03 & 2.65 & 0.40 \\
PCWP (mmHg) & 9.27 & 3.19 & 10.02 & 3.62 & 0.29 \\
SAP (mmHg) & 111.09 & 14.94 & 113.45 & 19.90 & 0.52 \\
MAP (mmHg) & 73.11 & 10.89 & 72.65 & 14.10 & 0.86 \\
DAP (mmHg) & 56.65 & 9.59 & 55.37 & 10.77 & 0.55 \\
HR (beats min-1) & 72.30 & 10.44 & 76.20 & 11.72 & 0.09 \\
CI l (min-1 m-2) & 2.43 & 0.61 & 2.78 & 0.58 & 0.01 * \\
SV (ml) & 66.65 & 17.31 & 74.17 & 20.03 & 0.06 \\
SVI (ml m-2) & 33.53 & 7.26 & 37.12 & 8.78 & 0.04 * \\
SVRI (dyne s cm-5 m $^{-2}$ ) & 2329.90 & 607.06 & 1982.80 & 516.72 & $0.00 \star$ \\
\hline
\end{tabular}

Abbreviations. SVV: stroke volume variation; CVP: central venous pressure; PCWP: pulmonary capillary wedge pressure; SAP: systolic arterial pressure; MAP: mean arterial pressure; DAP: diastolic arterial pressure; HR: heart rate; CI: cardiac index; SV: stroke volume; SVI: stroke volume index; SVRI: systemic vascular resistance index. Group 1: responders (gain in stroke volume index of $5 \%$ or more from baseline value after fluid therapy; Group 2: non-responders. Values are mean \pm standard deviation. $* \mathrm{P}<0.05$ was considered a significant difference. 
Table 3. Hemodynamic data after fluid therapy in patients of Group 1

\begin{tabular}{lccccc}
\hline & \multicolumn{2}{c}{ Before fluid therapy } & \multicolumn{2}{c}{ After fluid therapy } & P value \\
& Mean & SD & Mean & SD & \\
\hline SVV (\%) & 9.66 & 4.34 & 7.51 & 3.43 & 0.01 * \\
CVP (mmHg) & 5.61 & 2.19 & 6.60 & 2.37 & 0.04 * \\
PCWP (mmHg) & 9.27 & 3.19 & 10.11 & 3.34 & 0.22 \\
SAP (mmHg) & 111.09 & 14.94 & 116.42 & 14.99 & 0.09 \\
MAP (mmHg) & 73.11 & 10.89 & 75.02 & 11.06 & 0.40 \\
DAP (mmHg) & 56.65 & 9.59 & 57.14 & 9.43 & 0.80 \\
HR (beats min-1) & 72.30 & 10.44 & 73.07 & 10.45 & 0.72 \\
CI $1\left(\mathrm{~min}^{-1} \mathrm{~m}^{-2}\right)$ & 2.43 & 0.61 & 2.82 & 0.78 & $0.01 *$ \\
SV (ml) & 66.65 & 17.31 & 76.99 & 22.57 & $0.01 *$ \\
SVI (ml m-2) & 33.53 & 7.26 & 38.68 & 9.60 & $0.00 *$ \\
SVRI (dyne s cm-5 $\mathrm{m}^{-2}$ ) & 2329.90 & 607.06 & 2054.58 & 544.05 & $0.02 *$ \\
\hline
\end{tabular}

Abbreviations. SVV: stroke volume variation; CVP: central venous pressure; PCWP: pulmonary capillary wedge pressure; SAP: systolic arterial pressure; MAP: mean arterial pressurc; DAl: diastolic arterial pressure; HR: heart rate; CI: cardiac index; SV: stroke volume; SVI: stroke volume index; SVRI: systemic vascular resistance index. Group 1: responders (gain in stroke volume index of 5\% or more from baseline value after fluid therapy $(n=47)$. Values are mean \pm standard deviation. $\star P<0.05$ was considered a sigmificant difference.

Table 4. Hemodynamic data after fluid therapy in patients of Group 2

\begin{tabular}{lccccc}
\hline & \multicolumn{2}{c}{ Before fluid therapy } & \multicolumn{2}{c}{ After fluid therapy } & P value \\
& Mean & SD & Mean & SD & \\
\hline SVV (\%) & 7.57 & 2.96 & 6.11 & 2.61 & 0.02 * \\
CVP (mmHg) & 6.03 & 2.65 & 7.01 & 2.88 & 0.10 \\
PCWP (mmHg) & 10.02 & 3.62 & 11.12 & 3.62 & 0.15 \\
SAP (mmHg) & 113.45 & 19.90 & 117.37 & 18.65 & 0.34 \\
MAP (mmHg) & 72.65 & 14.10 & 73.91 & 13.58 & 0.67 \\
DAP (mmHg) & 55.37 & 10.77 & 55.74 & 9.93 & 0.87 \\
HR (beats min-1) & 76.20 & 11.72 & 77.77 & 11.39 & 0.52 \\
Cl 1 (min-1 m-2) & 2.78 & 0.58 & 2.78 & 0.59 & 0.96 \\
SV (ml) & 74.17 & 20.03 & 71.85 & 18.44 & 0.57 \\
SVI (ml m-2) & 37.12 & 8.78 & 35.94 & 7.90 & 0.51 \\
SVRI (dyne s cm & 1982.80 & 516.72 & 1991.89 & 514.39 & 0.93 \\
\hline
\end{tabular}

Abbreviations. SVV: stroke volume variation; CVP: central venous pressure; PCWP: pulmonary capillary wedge pressure; SAP: systolic arterial pressure; MAP: mean arterial pressurc; DAP: diastolic arterial pressure; HR: heart rate; CI: cardiac index; SV: stroke volume; SVI: stroke volume index; SVRI: systemic vascular resistance index. Group 2: non-responders (gain in stroke volume index of less than 5\% from baseline value after fluid therapy $(\mathrm{n}=45)$. Values are mean \pm standard deviation. $\star \mathrm{P}<0.05$ was considered a significant difference. 


\section{DISCUSSION}

The results of the present study show that pulse contour-derived variations in stroke volume were significantly correlated with changes in cardiac index, thus indicating the increase in cardiac preload due to volume loading. Furthermore, SVV was predictive of cardiac responsiveness to fluid therapy. In contrast, routinely used static variables of cardiac preload, such as CVP and PCWP, failed to predict volume responsiveness.

Stroke volume variation occurs due to cyclic changes of intrathoracic pressure induced by mechanical ventilation. An increase in intrathoracic pressure during the inspiratory phase leads to a temporary reduction in cardiac preload and therefore to a temporary reduction in stroke volume. In the presence of hypovolaemia, the left ventricle operates on the ascending part of the Frank-Starling curve and changes in $\mathrm{SV}$ are more pronounced than during normovolaemia. During fluid therapy, there is a rightward shift of the left ventricular function on the Frank-Starling curve, corresponding to the observed decrease of SVV. SVVs result in concomitant changes of arterial pressure, which can be assessed by measurement of SPV or PPV. In a recent systematic review, it was concluded that these dynamic measures of preload responsiveness were superior to the static preload parameters CVP or PCWP. The review acknowledged that there were still few studies validating dynamic parameters. ${ }^{.}$Previous studies demonstrated the ability of SVV to predict fluid responsiveness in patients during sepsis state ${ }^{21}$ or undergoing neurosurgery, ${ }^{11}$ but inconsistent results were found in cardiac surgery patients. ${ }^{16,18}$ Our current study in 92 postoperative cardiac surgical patients clearly demonstrated that SVV is a good predictor of fluid responsiveness, and as such far better than static parameters of preload.

This study suggests that a SVV of $9.6 \pm 4.3 \%$ will predict positive fluid responsiveness, defined as an increase in SVI by $5 \%$ or more. By using ROC analysis, it was shown that not only was the predictive value of SVV superior to the predictive value of CVP and PCWP, but also that the area under the curve indicates these static preload measures are not better than random guess. The results from our study confirm the previously proposed threshold values to discriminate between responders and non-responders. In patients with SVV values of $7.6 \pm 3 \%$, an increase in SVI by $5 \%$ or more cannot be expected. This is of evident clinical interest to reduce needless fluid therapy.

The results of the present investigation show the same pattern as most of the previously performed studies on SVV. However, comparison of SVV studies is difficult, mainly because of the following five reasons: differences in ventilator setting, in cardiac function, in volume load, in the amount of SVI increase considered a positive response and differences in the equipment used for analysing SVV. It was reported that SVV values were greater when using large tidal volumes $\left(\mathrm{TV}_{\mathrm{i}}\right)$, both during volume responsiveness and after volume loading. ${ }^{22}$ In our study, the ${ }^{\top} \mathrm{Tv}_{\mathrm{i}}$ was 
set at $6 \mathrm{~mL} \mathrm{~kg}{ }^{-1}$ ideal body weight and the PEEP at $8 \mathrm{~cm} \mathrm{H}_{2} \mathrm{O}$. It is our standard hospital practice to provide lung protective ventilation in most of our patients, applying these ventilator settings. In previous work, higher $T v_{i} s\left(7-15 \mathrm{~mL} \cdot \mathrm{kg}^{-1}\right)$ were used and also higher baseline SVVs (13.6-19.9\%) were observed. ${ }^{16}$ These higher $\mathrm{Tv}_{\mathrm{i}} \mathrm{s}$ may explain the apparently conflicting differences between these results of earlier studies and the average baseline SVV value of $8.6 \%$ in our study. It was shown that SVV is also potentially influenced by a compromised left ventricle's ability to use the Frank-Starling mechanism. The predictive value of SVV as a marker of fluid responsiveness was less in patients with reduced cardiac function. ${ }^{23}$ In the present study, patients were studied after cardiac surgery with cardiopulmonary bypass. Although all our study patients had an ejection fraction of $40 \%$ or more before surgery, it is clear that the postischemic contractile dysfunction in these patients often resulted in much lower ejection fractions at the time of the investigation. Therefore, it is difficult to compare the postoperative cardiac surgical patients with moderate left ventricular functions to studies that analyse data from patients with good left ventricles. We have arbitrarily defined both the volume load that was used $(10 \mathrm{ml}$ * $\mathrm{BMI}$ of a plasma expander) as well as what was considered to be a positive response to a fluid load (increase of SVI of $5 \%$ or more of its previous value). This choice was made in order to obtain data comparable to findings from similar research. Volume load in previous studies ranged between $7-10 \mathrm{ml} . \mathrm{kg}^{-1}$ and $10-20 \mathrm{ml}$ * BMI and a positive response to a fluid load ranged between 5-15\% increase in SVI. ${ }^{13,16-18,211}$ From our data it can be concluded that the SVV variable is as good predictor of the cardiac responsiveness to even a small volume load, which is of course of utmost clinical importance. Most previous research on SVV was performed with the PiCCO system, while in the present study the LiDCO system was used. These recently introduced devices both continuously analyse the arterial waveform, based on an algorithm first described by Wesseling and co-workers, and allow monitoring of beat-to-beat stroke volumes. ${ }^{21}$ Our study indicates that the LiDCO system is also an accurate device to measure SVV and to predict fluid responsiveness, but comparison of these two devices is beyond the scope of this investigation.

Our findings are of particular interest given the patient population studied. In these patients after coronary artery surgery, it is well-recognised that LV ejection fraction is usually reduced significantly. ${ }^{2-4}$ The overuse of catecholamines in this setting without adequate volume resuscitation may result in tissue ischemia. Conversely, the excessive use of fluids without resultant increase in cardiac output may cause edema and contribute to further tissue injury and organ dysfunction. In this vulnerable period after cardiac surgery where precise hemodynamic management is warranted, CVP and PCWP are insensitive preload markers and sometimes misleading. SVV on the other hand enabled prediction of cardiac responsiveness to fluid therapy and assessment of the hemodynamic effects of volume loading. 
The clinical use of these functional hemodynamic parameters has certain limitations: these methods may be used only in mechanically ventilated patients with no arrhythmias, whose arterial pressure is monitored invasively and under relatively stable conditions as described in our methods section.

There is no single parameter which can give us under all circumstances in all patients the information whether the muscle fibers of the left ventricle are still in the volume-dependent part of the Starling curve or have already reached their optimal length, the so-called preload. Clinical expertise and the use of several different parameters are necessary for precise titration of fluid therapy and cardiovascular management in daily practice. The limited value of CVP and PCWP as preload markers, the ungoing debate regarding the safety and efficacy of pulmonary artery catheters, the search for cheaper, continuous and less-invasive monitor systems are all factors contributing to the renewed interest in arterial pulse contour analysis, a technique first described in 1930. Continuous arterial waveform analysis has been extensively validated as a method for measuring continuous cardiac output. ${ }^{25}$ The pulse contour monitoring systems give the benefits of continuous numerical SVV as well as continuous SV measurements and other variables not reported in this study. SVV can predict fluid responsiveness and allows real-time assessment of the cardiovascular effects of volume loading. Therefore, together with continuous cardiac output measurements, on-line SVV monitoring helps to optimise hemodynamic conditions in ventilated high-risk patients after cardiac surgery.

In summary, our results strengthen the importance of functional hemodynamic monitoring. SVV is a good preload parameter for predicting and for on-line monitoring of cardiac responsiveness to fluid therapy. This functional preload marker is far superior to the static parameters CVP and PCWP. 


\section{REFERENCES}

1. Kumar A, Anel R, Bunnell E, Habet K, Zanotti S, Marshall S, Neumann A, Ali A, Cheang M, Kavinsky C, Parrillo JE. Pulmonary artery occlusion pressure and central venous pressure fail to predict ventricular filling volume, cardiac performance, or the response to volume infusion in normal subjects. Crit Care Med 2004;32:691-699.

2. Cheung A, Savino J, Weiss S, Aukburg S, Berlin J. Echocardiographic and hemodynamic indexes of left ventricular preload in patients with normal and abnormal ventricular function. Anesthesiology 1994;81:376-387.

3. Sakka S, Bredle D, Reinhart K, Meier-Hellmann A. Comparison between intrathoracic blood volume and cardiac filling pressures in the early phase of hemodynamic instability of patients with sepsis or septic shock. J Crit Care 1999;14:78-83.

4. Perel A, Pizov R, Cotev $S$. The systolic pressure variation is a sensitive indicator of hypovolemia in ventilated dogs subjected to graded hemorrhage. Anesthesiology 1987;67:489-502.

5. Perel A. Assessing fluid responsiveness by the systolic pressure variation in mechanically ventilated patients. Anesthesiology 1998;89:1309-1310.

6. Rooke $G$, Schwid $H$, Shapira $Y$. The effect of graded hemorthage and intravascular volume replacement on systolic pressure variation in humans during mechanical and spontaneous ventilation. Anesth Analy 1995;80:925-932.

7. Tavernier B, Makhotine O, Lebuffe G, Dupont J, Scherpereel P. Systolic pressure variation as a guide to fluid therapy in patients with sepsis-induced hypotension. Anesthesiology 1998;89:1313-1321.

8. Michard F, Teboul J. Predicting fluid responsiveness in ICU patients. Chest 2002;121:2000-2008.

9. Pinsky M. Functional haemodynamic monitoring. Intensive Care Med 2002;28:386-388.

10. Kramer A, Zygun D, Hawes H, Easton P, Ferland A. Pulse pressure variation predicts fluid responsiveness following coronary artery bypass surgery. Chest 2004;126:1563-1568.

11. Berkenstadt H, Margalit N, Hadani M, Friedman Z, Segal E, Villa Y, Perel A. Stroke volume variation as a predictor of fluid responsiveness in patients undergoing brain surgery. Anesth Analg 2001;92:984-989.

12. Preisman S, Kogan S, Berkenstadt H, Perel A. Predicting fluid responsiveness in patients undergoing cardiac surgery: functional haemodynamic parameters including the Respiratory Systolic Variation Test and static preload indicators. Br J Anaesth 2005;95:746-755.

13. I-Iofer C, Mueller S, Ferrer L, Klaghofer R, Genoni M, Zollinger A. Stroke volume and pulse pressure variation for prediction of fluid responsiveness in patients undergoing off-pump coronary artery bypass grafting. Chest 2005; $128: 848-854$.

14. Michard F, Teboul J. Using heart-lung interactions to assess fluid responsiveness during mechanical ventilation. Crit Care 2000;4:282-289.

15. Wesseling $\mathrm{K}$, de Wit $\mathrm{B}$, Weber J, Smith $\mathrm{N}$. A simple device for the continuous measurement of cardiac output. Adv Cardiovasc Physiol 1983;5:16-52.

16. Reuter D, Felbinger T, Schmidt C, Kilger E, Goedje O, Lamm P, Goetz A. Stroke volume variation for assessment of cardiac responsiveness to volume loading in mechanically ventilated patients after cardiac surgery. Intensive Care Med 2002;28:392-398.

17. Reuter D, Felbinger T, Kilger E, Schmidt C, Lamm P, Goetz A. Optimizing fluid therapy in mechanically ventilated patients after cardiac surgery by on-line monitoring of left ventricular stroke volume variations. Comparison with aortic systolic pressure variations. $\mathrm{Br} \mathrm{J}$ Anaesth 2002;88:124-126.

18. Wiesenack C, Prasser C, Roedig G, Keyl C. Stroke volume variation as an indicator of fluid responsiveness using pulse contour analysis in mechanically ventilated patients. Anesth Analg 2003;96:1254-1257. 
19. Rex S, Brose S, Metzelder S, Hueneke R, Schaclte G, Autschbach R, Rossaint R, Buhre W. Prediction of fluid responsiveness in patients during cardiac surgery. Br J Anaesth 20(1);93:782788.

20. Wiesenack C, Fiegl C, Keyser A, Prasser C, Keyl C. Assessment of fluid responsiveness in mechanically ventilated cardiac surgical patients. Eur J Anaesthesiol 2005;22:658-665.

21. Marx G, Cope T, McCrossan L, Swaraj S, Cowan C, Mostafa S, Wenstone R, Leuwer M. Assessing fluid responsiveness by stroke volume variation in mechanically ventilated patients with severe sepsis. Eur J Anaesthesiol 2004;21:132-138.

22. Reuter D, Bayerlein J, Goepfert M, Weis F, Kilger E, Lamm P, Goetz A. Influence of tidal volume on left ventricular stroke volume variation measured by pulse contour analysis in mechanically ventilated patients. Intensive Care Med 2003;29:476-480.

23. Reuter D, Kirchner A, Felbinger T, Weis F, Kilger E, Lanm P, Goetz A. Usefulness of left ventricular stroke volume variation to assess fluid responsiveness in patients with reduced cardiac function. Crit Care Med 2003;31:1399-1404.

24. Breisblatt W, Stein K, Wolfe C, Follansbee W, Capozzi J, Armitage J, Hadersty R. Acute myocardial dysfunction and recovery: a common occurrence after coronary bypass surgery.J Am Coll Cardiol 1990;15:1261-1269.

25. Goedje O, Hoeke K, Lichtwarck-Aschoff M, Falthauser A, Lamm P, Reichart B. Continuous cardiac output by fensoral arterial thermodilution calibrated pulse contour analysis: comparison with pulmonary arterial thermodilution. Crit Care Med 1999;27:2407-2412. 


\section{CHAPTER 9}

\section{Shortening ICU stay after coronary artery surgery}

John H. Heijmans ${ }^{1}$, MD, MS; Ghislaine A.P.G. van Mastrigt ${ }^{2}$, MS; Jos G. Maessen ${ }^{2}, \mathrm{MD}, \mathrm{PhD}$ and Paul M.H.J. Roekaerts ${ }^{13}, \mathrm{MD}, \mathrm{PhD}$

${ }^{1}$ Department of Anesthesiology, ${ }^{2}$ Department of Cardiothoracic Surgery,

${ }^{3}$ Department of Intensive Care

Submitted for publication.

In part published in Critical Care medicine 2006; 34: 65-75 


\section{ABSTRACT}

Study Objective: We recently published a randomized clinical trial comparing safety and cost-effectiveness in 600 patients undergoing either short-stay intensive care ( 8 hours of intensive care treatment) or control treatment (care as usual, overnight intensive care treatment) after coronary artery surgery. The reasons why in some patients in the short stay group discharge from the ICU within 8 hours failed, are now described.

Design: Retrospective analyzis by an investigational team of 6 experts working in the field of cardiac surgery, anesthesiology and cardiac critical care of the data of patients in which rapid discharge failed.

Setting: Perioperatively in low-risk coronary artery surgery patients.

Paticnts: 137 patients in the short-stay ICU group in whom rapid discharge failed.

Interventions: Nothing was done with these 137 patients on behalf of this study.

Measurements: Three research questions were formulated before analyzing the data: What was the primary factor responsible for the delayed discharge? Could this factors' effect on discharge have been avoided? Could a small change in the SSIC protocol be made so that these factors in the future no longer delay discharge from the ICU? Answers were formulated within the investigational team by concensus.

Main Results: Weaning from the ventilator was not initiated in time in 55/137 patients because of intercurrent problems (ischemic: 12; low cardiac output: 7 hypertension: 7; hypotension: 8; major dysrhythmias: 3; excessive bleeding: 17). Weaning from the ventilator was initiated in time but rapid weaning failed in 65/137 patients (pulmonary factors: 14 ; decreased arousal: 28 ; restless agitation: 23 ) Rapid discharge failed due to logistic reasons in $18 / 137$ patients.

In was concluded by the investigators that, in retrospect, $38 / 137$ patients could have been successfully discharged within 8 hours after arrival in the ICU: 4 in the ischemic group, 3 in the hypertension group, 2 in the hypotension group, 5 in the bleeding group, 10 in the pulmonary factors group and 14 in the logistics group.

Conclusions: Recommendations are made how to further improve the success rate of this safe and cost-effective short-stay ICU treatment by developing a Clinical Pathway Protocol and guidance of this protocol by a Continuous Quality Improvement Team. 


\section{INTRODUCTION}

We recently published a randomized clinical trial on safety and cost-effectiveness of short-stay intensive care (SSIC) after coronary artery surgery in 600 lowrisk surgical patients.' Patients were randomly assigned to undergo either SSIC treatment ( 8 hours of intensive care treatment) or control treatment (care as usual, overnight intensive care treatment). We concluded that discharge from the ICU to a step-down unit within eight hours after the end of surgery is a safe and cost-effective approach as compared to the common practice of overnight ICU treatment.

In the SSIC group, 137 patients could not be discharged timely from the ICU. There were various reasons for the delayed discharge, but these were not analyzed in-depth in that study. Understanding the factors which prevent early discharge are of imminent importance for the further clinical development of SSIC. The aim of the present investigation was to analyze the different factors which prevented rapid discharge from the ICU and to study whether these factors could have been prevented and/or whether corrections could be made in the SSIC protocol so that these factors in the future could no longer prevent rapid discharge from the ICU. From these data, important recommendations could de made for the development of a Clinical Pathway Protocol that allows rapid transfer of low-risk coronary artery surgical patients out of the ICU.

\section{METHODS}

The safety and cost-effectiveness of short-stay intensive care (SSIC) treatment for low-risk coronary artery surgery patients was evaluated in a recently published randomized clinical equivalence trial. A total of 600 patients were randomly assigned to undergo either SSIC treatment ( 8 hours of intensive care treatment) or control treatment (care as usual, overnight intensive care treatment). The primary outcomes measures were intensive care readmissions and total hospital stay. The secondary outcome measures were total hospital costs, quality of life, postoperative morbidity, and mortality. Hospital costs consisted of the cost of hospital admission or admissions and outpatient costs.

We now retrospectively analyzed the hospital charts of the 137 SSIC patients in whom discharge out of the ICU within 8 hours failed for various reasons. The investigation team which analyzed the data consisted of four investigators from the RCT (GvM, JH, PR, JM) and two experienced cardiac anesthesiologistsintensivists, not primarily involved in the RCT. Three research questions were formulated before analyzing the data and the answers were formulated after concensus within the team:

1. What was the primary factor responsible for the delayed discharge?

2. Could this factors' effect on discharge have been avoided? 
3. Could a small change in the SSIC protocol be made so that these factors no longer delay discharge from the ICU?

The night before surgery, all patients received lorazepam orally as sleep medication. The dose was individualized according to age and body weight $(1-2.5 \mathrm{mg})$. The morning of surgery, all patients received midazolam orally as premedication. The dose was individualized according to age and body weight (3.75-7.5 mg). Anaesthesia management was standardized and consisted of continuous infusions of propofol 1-2 mg. $\mathrm{kg}^{-1} \cdot \mathrm{h}^{-1}$ and 1-2 $\mu \mathrm{g} \cdot \mathrm{kg}^{-1} \cdot \mathrm{min}^{-1}$ alfentanil. ${ }^{2.3}$ In the ICU, for pain relief, the opioid piritramide was given $1 \mathrm{mg} \cdot \mathrm{h}^{-1}$ until discharge and paracetamol rectally was started 4 times daily $1000 \mathrm{mg}$. No NSAIDs were given because of their potential detrimental effect on the myocardium Propofol $1 \mathrm{mg} \cdot \mathrm{kg}^{-1} \cdot \mathrm{h}^{-1}$ was continued in the ICU if the patients' core temperature was below $37^{\circ} \mathrm{C}$ for thermal distress and was stopped when patients' were rewarmed until $37^{\circ} \mathrm{C}$. ${ }^{+}$The dose was adjusted, if necessary, in small steps to provide optimal patient comfort. Weaning from the ventilator was protocol driven and completely managed by the ICU-nurses (Figure 1). Criteria for discharge of patients from the ICU to a stepdown unit (cardiothoracic medium care unit) are listed in Table 1.

ICU treatment included: (1) continuous monitoring of vital parameters, including data from arterial lines and central venous lines (often pulmonary catheters in more complex cases, Swan-Ganz, Edwards Lifesciences, Irvine, CA, USA); (2) always a dedicated ICU doctor on the ward and full-time supervision of treatment by a qualified intensivist; (3) nurse-patient ratio mostly $1: 1$ and vital parameters registered by nurses every $15 \mathrm{~min}$ or every $30 \mathrm{~min}$ or every $60 \mathrm{~min}$, as dictated by protocol; (4) possibility for all types of mechanical ventilation and cardiovascular support and experience with all types of monitoring devices.

MCU treatment included: (1) continuous monitoring of most vital parameters (ECG, peripheral saturation, respiratory rate, non-invasive automated blood pressure measurements at the set intervals) and, although not routinely applied, possibility for monitoring with arterial lines and central venous lines (central venous pressure); (2) During day hours, a cardiothoracic registrar in the MCU supervised by a cardiothoracic surgeon and during night hours, this team is out of the hospital but available on-call.; (3) nurse-patient ratio mostly $1: 2$ and vital parameters registered by nurses every $60 \mathrm{~min}$ or every $120 \mathrm{~min}$, as dictated by protocol; (4) possibility for oxygen therapy.

\section{RESULTS}

The difference in intensive care readmission between the two groups of $1.13 \%$ was very small and not significantly different $(\mathrm{p}=0.241 ; 95 \%$ confidence interval, $0.9 \%$ to $2.9 \%$ ). The total hospital stay ( $\mathrm{p}=0.807 ; 95 \%$ confidence interval, 1.2 to 0.4 ) and postoperative morbidity were comparable between the groups. The SSIC 


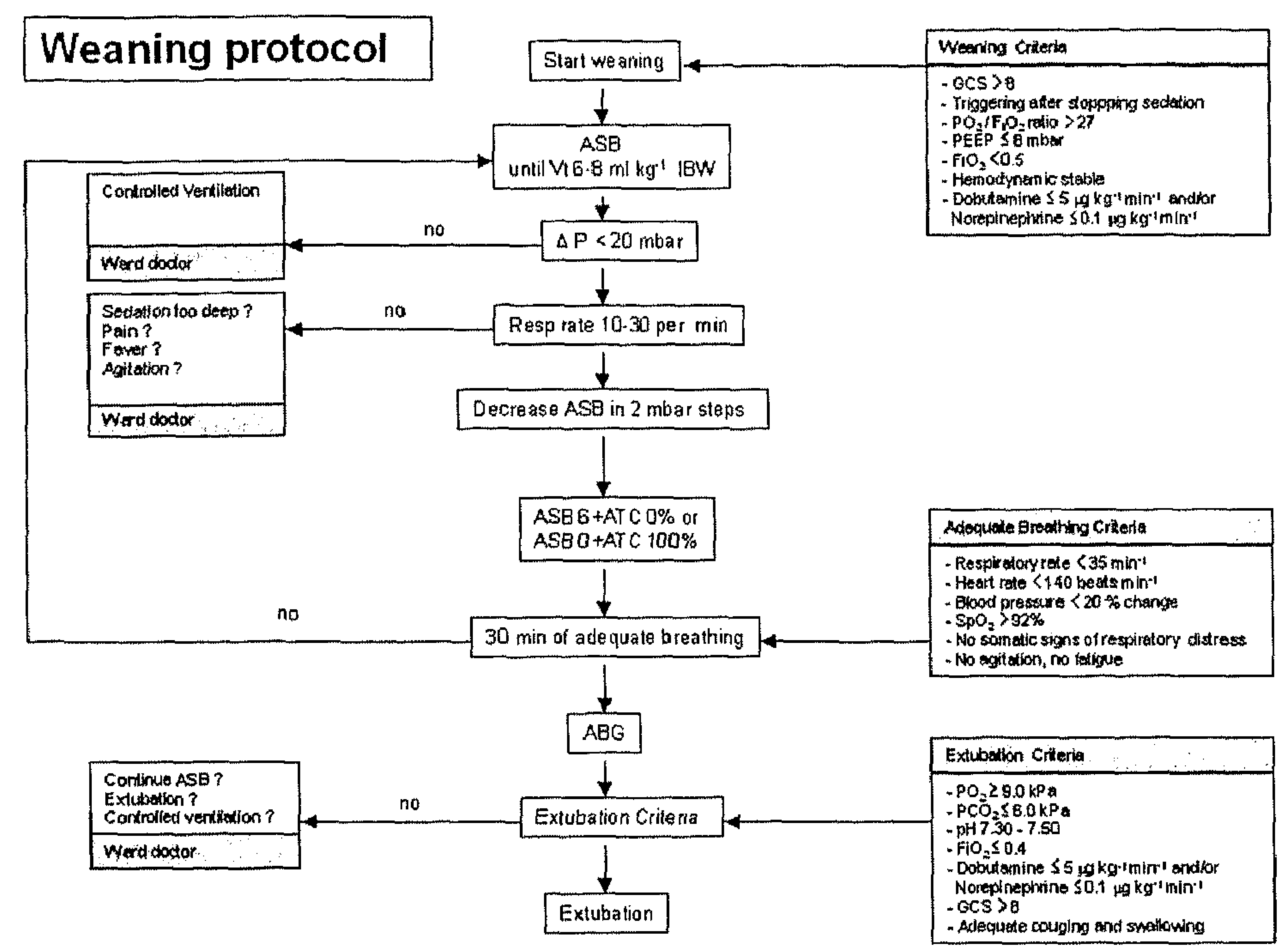

Figure 1. The weaning protocol for low-risk coronary artery surgery patients.

Abbreviations. ASB: assisted spontaneous breathing; Vt = tidal Volume; IBW; Ideal Body Weight; DeltaP: driving pressurc; ATC: automatic tube compensation.

group's quality of life improved more compared with the control group's quality of life $(p=0.0238 ; 95 \%$ confidence interval, 0.0012 to 0.0464$)$. The total hospital costs for SSIC were significantly lower (95\% confidence interval, $-€ 1,581$ to $-€$ 174) compared with those for the control group ( $€ 4,625$ and $€ 5,441$, respectively). The estimated incremental cost-effectiveness ratio (cost/delta quality-adjusted life months) thus showed the dominance of SSIC. Bootstrap and sensitivity analyses confirm the robustness of the study findings.

Retrospectively, the charts of the 137 patients which could not be discharged from the ICU within eight hours were analyzed. It was concluded that there were basically three categories of patients: (1) weaning from the ventilator was not initiated in time because of intercurrent problems; (2) weaning from the ventilator was initiated in time but rapid weaning failed; and, (3) rapid discharge failed due to logistic reasons. A further subdivision of these three categories of patients was made and 10 patient groups were formulated according to the most important factor responsible for delayed discharge from the ICU (Table 2). 
Table 1. ICU discharge criteria

\begin{tabular}{|c|c|}
\hline Pulmonary & $\begin{array}{l}\text { Extubation } \geq 30 \text { min } \\
\text { Oxygen } \leq 51 \mathrm{~min}^{-1} \text { nasally } \\
\text { Respiratory rate }=10 \mathrm{~min}^{-1} \text { and } \leq 25 / 30 \mathrm{~min}^{-1} \\
\mathrm{PaO}_{2}>9 / 10 \mathrm{kPa}^{-1} \mathrm{PCO}_{2}<6 \mathrm{kPa} \text { (or less than } 25 \% \text { deviation from preop values) }\end{array}$ \\
\hline Cardiac & $\begin{array}{l}\text { No myocardial ischemia or ongoing infarction } \\
\text { No hemodynamically significant dysrhythmias }\end{array}$ \\
\hline Fluid balance & $\begin{array}{l}\text { Chest tube drainage } \leq 100 \mathrm{~mL} \cdot \mathrm{h}^{-1} \\
\text { Diuresis }>0.5 \mathrm{~mL} \cdot \mathrm{kg}^{-1} \cdot \mathrm{h}^{-1}\end{array}$ \\
\hline Neurologic & No signs/symptoms of major neurologic complication \\
\hline Hemodynamic & $\begin{array}{l}\text { No jv vasoactive drugs, except dopamine } 2 \text { and/or nitroglycerine } 0.5 \mu \mathrm{g} \cdot \mathrm{kg}^{-1} \cdot \mathrm{min}^{-1} \\
\text { No IABP } \\
\text { Cardiac Index }>2 \mathrm{~L} \cdot \mathrm{min}^{-1} \cdot \mathrm{m}^{-2} \text { [if PAC is inserted] }\end{array}$ \\
\hline
\end{tabular}

Table 2. Reasons for failed early discharge from the ICU

\begin{tabular}{llc}
\hline I. No weaning initiated & & 12 \\
1. Cardiovascular & ischemic & 7 \\
& low cardiac output & 7 \\
& hypertension & 8 \\
& hypotension & 3 \\
& major dysrhytmias & 17 \\
2. Surgical/Hematologic & excessive bleeding & \\
II. Failed rapid weaning & & 14 \\
1. Pulmonary factors & & 28 \\
2. Decreased arousal & & 23 \\
3. Restless agitation & & 18 \\
III. Logistics & \\
\hline
\end{tabular}

In was concluded by the investigators that $38 / 137$ patients could have been succesfully discharged within 8 hours after arrival in the ICU: 4 in the ischemic group, 3 in the hypertension group, 2 in the hypotension group, 5 in the bleeding group, 10 in the pulmonary factors group and 14 in the logistics group.

In the pulmonary factors group mean extubation time was $450 \pm 228 \mathrm{~min}$, in the decreased arousal group $637 \pm 166 \mathrm{~min}$ and in the restless agitation group 865 $\pm 186 \mathrm{~min}$. 


\section{DISCUSSION}

Routine overnight postoperative ventilation following cardiac surgery has been standard practice for several decades. 5 Justifications for this practice were: (1) the presumed beneficial effect of high-dose opioid anesthesia, which delayed weaning from artificial ventilation; (2) the frequent respiratory complications in the early postoperative phase $;^{7-14}$ (3) the increased work of breathing in spontaneously breathing patients would represent an added burden on the myocardium at a time when cardiac function may still be compromised; (4) physiologic stress may induce myocardial ischemia; (5) continuous intense postoperative analgo-sedation in combination with mechanical ventilation decreases myocardial ischemic episodes in the early postoperative phase. ${ }^{15}$

In recent years, the increasing number of patients undergoing cardiac operations under cardiopulmonary bypass and the limited resources for postoperative intensive care have stimulated attempts to reduce the length of stay in these highcost units. Indeed, cost containment and reduction without compromising quality of care have become major issues in cardiac surgery. One of the first measures taken was the early extubation of these patients. ${ }^{16-17}$ In recent years, more emphasis has been put on the early discharge of these patients from high-cost units.

Several reports in the literature, including our study, now suggest that early extubation and discharge from the ICU is safe and cost-effective. However, this practice has not yet become generalized, because some debate is still going on. ${ }^{18-31}$ Some authors found increased myocardial ischemia in fast-track patients and attributed this finding to increased activity of the sympathetic nervous system, increased myocardial oxygen demand and hemodynamic instability during this period. Other authors state that early extubation and discharge, apart from the economic benefit, have several clinical benefits, like improved ventricular performance, improved renal and respiratory function, early mobilization and faster return to normal nutrition. These authors suggest that early extubation and discharge should be the current standard of care after uncomplicated elective cardiac operations. As stated before by Meade, ${ }^{17}$ while research has not unequivocally established the safety of early extubation and discharge, serious adverse events are so low that a trial of thousands of patients (or, with even a combined morbidity endpoint, at least many hundreds of patients) would be required to definitively establish the effect. Given the widespread adoption of early extubation following cardiovascular surgery, and suggestions of cost-effectiveness, spending scarce resources on further studies to detect small but clinically important increases in morbidity following early extubation may not be warranted.

In the present study, after arrival in the ICU, patients had to be extubated within 7.5 hours and discharged within 8 hours to meet the criteria of short-stay intensive care treatment. For the purpose of the discussion, we define extubation times as early (within $7.5 \mathrm{~h}$ ), intermediate (within $12 \mathrm{~h}$ ) and late (after $12 \mathrm{~h}$ ). 
In general, it is not advisable to fast-track high-risk patients. Also, unexpected events in the operating room such as incomplete revasculanization or poor myocardial preservation and an inadequate or unstable cardiovacular performance postoperatively may dictate prolonged postoperative ventilation to reduce the work of breathing and to allow adequate analgo-sedation. ${ }^{32-37}$ Our first category of patients, where no early weaning from the ventilator was initiated, clearly fit into these types of problems. However, in the 'ischemic' group, 4 patients had discrete and aspecific ST-segment alterations compatible with pericarditis who were managed by the ward doctor and nurse as if they had myocardial ischemia. Just to be on the safe side, these patients were sedated longer. It is advized by the investigation team, that, whenever ST-segment alterations develop an intensivist must take the decision whether or not that specific patient can further be treated as a SSIC patient. In the 'hypertension' and 'hypotension' groups, 5 patients with minimal blood pressure instabilities were managed with continuous intravenous medications which necessitated longer invasive monitoring and thus were a contraindication for discharge. It is advised by the investigation team to administer in this setting more often sublingual, oral, rectal or IM medication, making discharge in this way possible. In the 'excessive bleeding' group, the investigation tean concluded that 5 patients were bleeding due to haematologic reasons. After pro-coagulant therapy, bleeding stopped immediately, although it was given at a late timepoint after surgery making rapid discharge impossible. It is advised by the investigation team give pro-coagulant therapy earlier in SSIC patients, but still only when indicated.

In our study, patients which could not be discharged from the ICU because of weaning problems were divided in to three groups. In the 'pulmonary factors' group mean extubation time was early $(7.5 \mathrm{~h})$, in the 'decreased arousal' group mean extubation time was intermediate $(10.5 \mathrm{~h})$ and in the 'restless agitation' group extubation was late $(15 \mathrm{~h})$.

Postoperative morbidity and mortality are, to a large extent, predictable based on preoperative status. ${ }^{38-39}$ Patients likely to have respiratory complications after cardiac surgery can be identified by the presence of heart failure, reoperation, emergency surgery, prior vascular operation, old age, impaired renal function and chronic obstructive pulmonary disease. Clearly, in our study, in some patients preoperative existing pulmonary problems were missed upon preoperative screening. These patients perhaps should not have been included in the study. Nevertheless, mean extubation time in these patients was early $(7.5$ hours after arrival). These patients were however not discharged because nurse and ward doctor believed that, on the basis of sequential suboptimal blood gas analyses, these patients would be better off in an ICU setting. Retrospectively, the investigation team thought that in $10 / 14$ patients SSIC treatment could have been performed with acceptance of suboptimal blood gas analyzis. The team advized that timely consultation of the intensivist in the future can prevent that these patients are kept 
to long in the ICU, as the MCU has all equipment and skills to further monitor these patients.

It has been recognized that the type of anesthetic-analgesic management during surgery and in the early postoperative period is a major determinant of early extubation and discharge. The debate whether one should use inhalational anesthesia or opioid anesthesia in this clinical setting is not yet settled. "We used a total intravenous anesthesia and analgo-sedation technique, as described in the methods section. When opioid anesthesia is used with the aim of reducing the sympathetic response to stress and the incidence of perioperative myocardial ischemia, early extubation is more difficult. In our intermediate extubation group, 28 patients (9.3\%) failed early extubation because of a decreased arousal which is usually described as "persistent anesthetic effect". Mean extubation time in this group was $637 \mathrm{~min}(10.5 \mathrm{~h})$ after arrival in the ICU. In this failed early extubation group, all patient were easily wakable, quiet, oriented, cooperative, painfree and calm. Left unstimulated, these patients closed their eyes, tried to sleep and had a very slow respiratory rate. Nevertheless, more than $90 \%$ of patients in the study population had no "persistent anesthetic effect". We therefore believe that our perioperative anesthesia-analgesia management is feasible in fast-tracking cardiac surgical patients. A possible advantage of our protocol is that it combines the allegedly beneficial effects of opiod anesthesia during the first postoperative hours, in which the incidence of myocardial dysfunction and ischemic episodes is highest, with relatively early extubation and discharge. Analysing the charts in the study population, revealed that the temperature was $35.4 \pm 0.7^{\circ} \mathrm{C}$ upon arrival in the ICU, that the time to rewarming thereafter to $37^{\circ} \mathrm{C}$ was $181 \pm 79 \mathrm{~min}$ and that propofol was given for $281 \pm 230 \mathrm{~min}$. The protocol dictated that propofol had to be stopped when then the patient's core temperature was $37^{\circ} \mathrm{C}$. Clearly, propofol was given almost 2 hours too long and there is clear evidence that this protocol violation was due to nurse-related factors. It can be assumed that stopping propofol infusion earlier, could have led to sooner extubation in some patients in this group. Keeping the patients warm or rewarming them in the operation room to $37^{\circ} \mathrm{C}$ and keeping them warm during transport to the ICU and installation in the ICU, is probably the single most important factor in making rapid weaning and early discharge from the ICU successful. Indeed, the faster the patient is warm, the sooner sedation can be stopped. The dose/administration of anesthetics-analgesics in the perioperative period is evidently the most important factor for delaying extubation time in this group. However, considering this as being the only factor is far too easy. Patient factors like personality-type and metabolism also affect the level of arousal after the administration of lorazepam, midazolam, propofol, alfentanil, and piritramide. These patient factors are clearly difficult to modulate.

Restless agitation in patients after stopping sedation was the reason for delayed discharge in 23 patients in the study population (7.5\%). These patients were restless, agitated, not communicative, not cooperative and stressed. The only therapeutic 
measure taken in this group was resedation with propofol and stopping sedation at regular intervals until patients were clear-headed and calm. Mean extubation time in this group was 15 hours after arrival in the ICU, the only group in which extubation was late. The etiology of this phenomenon is not clear, but certainly multifactorial. ${ }^{+1-51}$ We suggest that "encephalopathy" best describes the primary determinant for late extubation in this patient group. Encephalopathy is a wellrecognized complication of cardiac surgery. This delirium or encephalopathy is characterized by confusion, agitation, disorientation, slowness to regain consciousness after anesthesia, hyperautonomic states (rapid heart rate, jumps in blood pressure, sweating, and tremor), drowsiness, decreased alertness, memory deficit, or seizure but without obvious focal or localizing deficits. The incidence in the literature is $3-10 \%$. Cardiopulmonary bypass is associated with gaseous and particulate cerebral microembolisation, periods of hypoperfusion and a systemic inflammatory response syndrome (SIRS) contributing to cerebral edema. Therefore, CPB is considered as the major determinant of encephalopathy. In elderly patients, encephalopathy is more common. They are more sensitive to the effects of sensory deprivation, sedative-hypnotic drugs, electrolyte abnormalities, mild cerebral hypoxia, and the delayed effects of anesthesia. Other risk factors for encephalopathy include liver and renal disease, medications, chronic illness, cardiac failure, infections, history of stroke, hypertension, atrial fibrillation, diabetes, presence of a carotid bruit, $\mathrm{CPB}$ time, hypothermia and rewarming during $\mathrm{CPB}, \mathrm{pH}$-management during $\mathrm{CPB}$ and alcohol/anxiolytic drugs withdrawal states, pain, the deliriogenic effect of benzodiazepines, stress of surgery and anesthesia, sleep deprivation, anesthetic-analgesic drugs, and new environments. Regardless of these possible precipitating factors, the presence of vascular risk factors appears to be an important determinant of postoperative encephalopathy. Thus, apart from the triad microembolization-inflammation-hypoperfusion of the brain, patient factors play a pivotal role in the genesis of early postoperative encephalopathy. Although speculative, the personality type of the patient could, in part, also explain the restless agitation upon stopping the sedation after surgery. As early as 1939, the american psychiatrist Karen Horney described genetically-determined character traits underlying personality. Through the following decades, the NPA theory of personality has been further developed. ${ }^{51}$ It is interesting to think that, according to the NPA theory, the aggressive personality type shows restless agitation upon awakening after surgery, while, in contrast, the submissive type personality is more calm and cooperative after awakening in an unfamiliar environment. Because etiologic factors for encephalopathy or restless agitation are so numerous, it is difficult to advise how these problem could best be countered. Avoiding CPB or shortening CPB time, the use of in-line arterial filters, the use of membrane oxygenators and biocompatible tubings, optimal titration of hemodynamics, prudent thermoregulatory control and meticulous cannulation and surgery are all factors during surgery which could beneficially affect neurologic outcome. The anesthesiologist 
should titrate his anesthetic to the desired effect and avoid over-dosing. In the ICU, pain relief is crucial and all sedative-analgesic regimens should start with a basic analgesic infusion, supplemented with propofol to provide optimal patient comfort. This concept of providing analgesia-first, supplemented by sedation when needed has recently been proven effective in several studies. The ideal target comfort goal for most patients still appears to minimizing pain and maintaining a sleepy but arousable state which avoids coma and allows evaluations of pain and neurologic function. As therapeutic measures are currently limited, the importance of measures aimed at prevention should not be underestimated. Neuroprotective agents hold some promise, but none of these have been proven effective and safe so far. What impact this transient restless agitation has on long-term outcomes is currently unclear, but it may be a predictor of subsequent cognitive decline. Attempts to understand this phenomenon of restless agitation and to modify its outcome are warranted.

Most cardiovascular surgery is elective and a relatively small team of surgeons, perfusionists, anesthesiologists, intensivists and nurses are involved in caring for these patients. This should facilitate standardizing a number of aspects of care. However, changing from routine practice to early extubation and discharge requires education of, and cooperation from the entire medical and nursing staff. This team should develop a Clinical pathway Protocol. Care of patients identified for early extubation will differ in a number of ways, including altered preoperative education, adequate rewarming, titrated anesthesia-analgesia protocols, and motivation of all health workers involved. In our study, 18 patients could not be discharged early because of logistic problems. These included laboratory delay, human errors concerning the protocol, no step-down bed available and others. The investigation team concluded that $12 / 18$ failed discharge cases were however more or less due to a lack of interest or lack of belief in the project from individuals within the medical and nursing team involved. Also, a lack of ongoing information provided by the investigators to all health workers involved was at times a reason for failing discharge in these 12 patients. Therefore, a 'Continuous Quality Improvement Team' (1 cardiac anesthesiologist, 2 ICU nurses and 2 intensivists) was instituted recently to continuously motivate all health workers involved and to give the medical and nursing staff ongoing information, feed-back, and education.

We recently concluded that SSIC is a safe and cost-effective approach and that SSIC can be considered as an alternative for conventional postoperative intensive care treatment for low-risk coronary artery bypass graft patients. Other benefits include improved patient comfort, earlier mobilization, and more efficient resource utilization. Although it was previously argued that postoperative cardiac surgery patients could be treated out of the ICU in a recovery room, ${ }^{52-55}$ we strongly belief that an initial ICU setting is necessary for all coronary artery surgical patients and that a short period of optimal analgosedation with mechanical ventilation has numerous benefits in the early unstable postoperative phase, when patients still can 
suddenly deteriorate severely. Therefore, all ICU skills should be readily available in the immediate postoperative period. To our knowledge, most of the cardiac recoveries are located in a dedicated area within the ICU department, obviously for these safety issues.

In the present study, we described the patients in which early extubation and discharge from the ICU failed for different kinds of reasons. Recommendations are made how to further improve success rate. Developing a Clinical Pathway Protocol and guidance of this protocol by a Continuous Quality Improvement Team will definitely improve safety and efficacy of short-stay ICU treatment. 


\section{REFERENCES}

1. van Mastrigt A, Heijmans J, Severens J, et al. Short-stay intensive care after coronary artery bypass surgery: randomized clinical trial on safety and cost-effectiveness. Crit Care Med 2006;34:65-75.

2. Roekaerts PM, Gerrits HT, Timmerman BE, de Lange S. Continuous infusions of alfentanil and propofol for coronary artery surgery.J Cardiothorac Vasc Anesth 1995;9:362-367.

3. Roekaerts PM, de Lange S. Con: midazolam is not the sedative of choice to supplement narcotic anesthesia.J Cardiothorac Vasc Anesth. 1993;7:620-623.

4. Roekacrts PM, Huygen FJ, de Lange S. Infusion of propofol versus midazolam for sedation in the intensive care unit following coronary artery surgery. J Cardiothorac Vasc Anesth 1993;7:142147.

5. Dammann JF Jr, Thung N, Chistl L 2nd, Littefield JB, Muller WH Jr. The management of the severely ill patient after open heart surgery. Thorac Cardiovasc Surg 1963;45:80-90.

6. Lowenstein E, Hallowell P, Levine FH, Daggert WM, Austen WG, Laver MB. Cardiovascular responses to large doses of intravenous morphine in man. N Engl J Med 1969;281:1389-1393.

7. Thung N, Herzog P, Christlieb II, Thompson WM Jr, Dammann JF Jr. The cost op respiratory effort in postoperative cardiac patients. Circulation 1963;28:552-559.

8. Quasha AL, Loeber N, Feeley TW, Ullyot DJ, Roizen MF. Postoperative respiratory care: a controlled trial of early and late extubation following coronary artery bypass grafting. Anesthesiology 1980;52;135-141.

9. Shackford SR, Virgileo RW, Peters RM. Early extubation versus prophylactic ventilation in the high risk patient: a comparison of postoperative management in the prevention of respiratory complications. Anesth Analy 1981;60:70-80.

10. Johnson D, Thomson D, Mycyk T, Burbridge B, Mayers I: Respiratory outcomes with early extubation after coronary artery bypass surgery. J Cardiothorac Vasc Anesth 1997;64:706-709.

11. Shapiro BA, Vender JS, Peruzzi WT. Airway pressure therapy for cardiac surgical patients: state of the art and clinical controversies. J Cardiothorac Vasc Anesth 1992;6:735-748.

12. Ingersoll GL, Grippi MA. Preoperative pulmonary status and postoperative extubation outcome of patients undergoing elective cardiac surgery. Heart Lung 1991;20:137-143.

13. Jardin F, Farcot JC, Boisante L, Prost JF, Gueret P, Bourdarias JP. Influence of positive end-expiratory pressure on left ventricular performance. N Engl J Med 1981;304:387-392.

14. Lefemine AA, Harken DE. Postoperative care following open-heart operations: routine use of controlle ventilation. J Thorac Cardiovac Surg 1966;52:207-216.

15. Mangano D, Siliciano D, Hollenberg M, et al. The study of perioperative ischenia (SPI) research group. Postoperative myocardial ischemia: therapeutic trials using intnsive analgesia following surgery. Anesthesiology 1992;342-353.

16. Engelman RM, Rousou JA, Flack JE, et al. Fast-track recovery of the coronary bypass patient. Ann Thorac Surg 1994;58:1742-1746.

17. Meade $M O$, Gayatt $G$, Butler $R$, et al. Trials comparing early versus late extubation following cardiovascular surgery. Chest 2001:6:445S-453S.

18. Higgins TL. Pro: early endotracheal extubation is preferable to late extubation in patients following coronary artery surgery.J Cardiothorac Vasc Anesth 1992;6:488-493.

19. Cheng DC, Karski J, Peniston C, et al. Morbidity outcome in early versus conventional tracheal extubation after coronary artery bypass grafting: a prospective randomized controlled trial. Thorac Cardiovasc Surg 1996;112:755-764.

20. Berry PD, Thomas SD, Mahon SP, et al. Myocardial ischemia after coronary artery bypass grafting: early vs late extubation. Br J Anaesth 1998;80:20-25.

21. Silbert BS, Santamaria JD, O' Brien JL, Blyth CM, Kelly WJ, Molnar RR. Early extubation following coronary artery bypass surgery: a prospective randomized controlled trial: The fast track cardiac care team. Chest 1998;113:1481-1488. 
22. London $M$, Shroyer $A$, Jernigan $V$, et al. Fast-track cardiac surgery in a department of veterans affairs patient poulation. Ann Thorac Surg 1997;64:134-141.

23. Quigley RL, Reitknecht FL. A coronary artery bypass "fast-track" protocol is practical and realistic in a rural environment. Ann Thorac Surg 1997;64:706-709.

24. Siliciano D. Con: early extubation is not preferable to late extubation in patients undergoing coronary artery surgery. J Cardiothorac Vasc Anesth 1992;6:494-498.

25. Aps C. Fast-tracking in cardiac surgery. Br J Hosp Med 1995;54:139-142. Hall RI. Anesthesia for coronary artery surgery - a plea for a goal-directed approach. Can J Anaesth 1993;40:1178-1194.

26. Jones EL, Weintraub WS, Craver JM, Guyton RA, Cohen CL. Coronary bypass surgery. Is the operation different today? J Thorac Cardiovasc Surg 1991:101:108-115.

27. Edwards FH, Clark RE, Schwartz M. Coronary artery bypass grafting; the Society of Thoracic Surgeons National Database experience. Ann Thorac Surg 1994;57:12-19.

28. Butler J, Chong GL, Pillai R, Westaby S, Rocker GM. Early extubation after coronaryartery bypass surgery: effects on oxygen flux and hemodynamic variables. J Cardiovasc Surg 1992;33:276-280.

29. Klineberg PL, Geer RT, Hirsh RA, Aukburg SJ. Early extubation after coronary artery bypass graft surgery. Crit Care Med 1977;5:272-274.

30. Christakis GT, Ivanov J, Weisel RD, Birnbaum PL, David TE, Salemo TA. The changing pattern of coronary artery bypass surgery. Circulation 1989;80:1151-I161.

31. Hickey RF, Cason BA. Timing of tracheal extubation in adult cardiac surgery patients. J Card Surg 1995;10:340-348.

32. Slogoff S, Keats AS. Does perioperative myocardial ischemia lead to postoperative myocardial infarction? Anesthesiology 1995;62:107-114.

33. Snith R, Leung J, Mangano D. Postoperative myocardial ischemia in patients undergoing coronary artery bypass graft surgery. Anesthesiology 1991;74:464-473.

34. Mangano DT. Biventricular function after myocardial revascularization in humans: deterioration and recovery patterns during the first 24 hours. Anesthesiology 1985;62:571-577.

35. Stein KL, Breisblatt W, Wolfe C, Gasior T, Hardesty RL. Depression and recovery of right ventricular function after cardiopulmonary bypass. Crit Care Med 1990;18:1197-1200.

36. Leung JM, O'Kelly B, Browner WS, Tubau J, Hollenberg M, Mangano DT. Prognostic importance of postbypass regional wall-motion abnormalities in patients undergoing coronary artery bypass graft surgry. Anesthesiology 1989;71:16-25.

37. Gall SA, Olsen CO, Reves JG. Beneficial effects of endotracheal extubation on ventricular performance. J Thorac Cardiovasc Surg 1988;95:819-827.

38. Hammermeister KE, Burchfield C, Johnson R, Grover FL. Identification of patients at greatest risk for developing major complications at cardiac surgery. Circulation 1990;82:IV380-IV389.

39. Heijmans JH, Maessen JG, Roekaerts PM. Risk stratification for adverse outcome in cardiac surgery. Eur J Anaesthesiol 2003;20:515-527.

40. Tuman KJ, McCarthy RJ, Spiess BD, et al. Does choice of anesthetic agent significantly affect outcome after coronary surgery? Anesthesiology 1989;70:189-198.

41. Dumas A, Dupuis GH, Searle N, Cartier N. Early versus late extubation after coronary artery bypass grafting: effects on cognitive function.J Cardiothorac Vasc Anesth 1999;13:130-135.

42. Kelly BJ, Matthay MA. Prevalence and severity of neurologic dysfunction in critically ill patients. Influence on need for continued mechanical ventilation. Chest 1993;104:1818-1824.

43. McKhann GM, Grega MA, Borowicz ML, et al. Encepalopathy and stroke after coronary artery bypass grafting. Arch Neurol 2002;59:1422-1428.

44. Infeld B. Management of the elderly cardiac surgical patient: neurologic sequelae. Heart, Lung and Circulation 2004;13S:S19-S23.

45. McKhann GM, Grega MA, Borowicz LM Jr, Selnes OA. Stroke and encephalopathy after cardiac surgery: an update. Stroke 2006;37:562-571. 
46. Taggart PT, Westaby S. Neurological and cognitive disorders after coronary artery bypass grafting. Current Opinion in Cardiology 2001;16:271-276.

47. Gill R, Murkin JM. Neuropsychologic dysfunction after cardiac surgry: what is the problem? J Cardiothorac Vasc Anesth 1996;10:91-98.

48. Borowicz LM, Goldsborough MA, Selnes OA, McKhann GM. Neuropsychologic change after cardiac surgery : a critical review. J Cardiothorac Vasc Anesth 1996;10:105-112.

49. Stump DA, Rogers AT, Hammon JW, Newman SP. Cerebral emboli and cognitive outcome after cardiac surgery. J Cardiothorac Vasc Anesth 1996;10:113-119.

50. Llinas R, Barbut D, Caplan LR. Neurologic complications of cardiac surgery. Progresss in Cardiovascular Diseases 2000;43:101-112.

51. Benis AM. Toward self and sanity: on the genetic origins of the human character. Psychological dimensions, New York, 1985. ISBN 0884370747.

52. Chong JL, Grebenik C, Sinclair M, Fisher A, Pillai R, Westaby S. The effect of a cardiac surgical recovery area on the timing of extubation.J Cardiothorac Vasc Anesth 1997;11:474-480.

53. Chong JL, Pillai R, Fisher A, Grebenik C, Sinclair M, Westaby S. Cardiac surgery; moving away from intensive care. Br Heart J 1992;68:430-433.

54. Massey D, Meggit G. Recovery units: the future of post-operative cardiac care. Intensive Crit Care Nurs 1994;10:71-74.

55. Westaby $S$, Pillai $R$, Parry A, et al. Does modern cardiac surgery require conventional intensive care? Eur J Cardiothorac Surg $1993 ; 7: 313-318$. 
CHAPTER 10

Summary, Discussion and Conclusions 


\section{SUMMARY}

In the present thesis the development of a Clinical Pathway Protocol (CPP) for low - risk elective coronary artery surgery patients in the University Hospital of Maastricht is described. The aim was to outline a protocol that would define the best clinical practice for these patients, based on the principles of evidence-based medicine and taking into account economical aspects. Special emphasis was placed on the perioperative anaesthesiologal management and on the optimal use of all inhospital high-cost facilities such as the intensive care unit and the step-down facilities after surgery. The medical care according to the CPP included early postoperative extubation, rapid discharge within 8 hours from the ICU to a step-down unit and early hospital discharge. During the development of this protocol, several questions raised for which we could not find clear-cut answers in the literature. These unanswered questions led to the formulation of six research questions. Six studies were then performed in order to be able to describe a CPP that would incorporate cost-effective evidence-based best medical practice for the selected patient group. These six studies are presented in the present thesis.

In Chapter 2, "History of perioperative management in cardiac surgery", a brief overview is given of the development of perioperative anaesthetic management in cardiac surgery from the early fifties until present day. Over the years, there is an increase in medical demand from the public community as a whole and there is an ongoing growth of the available medical technology and possibilities that are offered to the public community. These factors added together, and facing an aging population, impose an intolerable financial burden on the shoulders of medical care providers and their governments. Governments must contain costs and resources. Economics are forced into the decision making process, a process that formerly used to belong to medical doctors alone. The consequence of this is that the medical field has to change in the way it used to treat its patients and to adapt to the new expectations and demands of the public and government. The task at hand is far from easy, the medical field has to do more with less. A new concept Evidence Based Medicine (EBM) was introduced with the purpose to base medical practice on best research findings eliminating subjective medical practice and thereby increasing cost-benefit ratios. The results from the conjunction of EBM with clinical expertise and patient values is then translated into hospital guidelines and protocols. The medical philosophy on how to treat patients best has changed from a medical specialist centered medical practice to a patient centered medical practice. A consequence of this is the establishment of so called clinical pathways. In this thesis, the development of a clinical pathway protocol (CPP) for low-risk elective coronary artery surgery patients is described. 
The aim was to describe a CPP only for low -risk coronary artery surgery patients. Therefore, it was necessary to be able to stratify patients into low-risk and high-risk patients. In Chapter 4, "Risk stratification for adverse outcome in cardiac surgery", we reviewed the literature for risk scoring and risk evaluating scoring systems that would help us in selecting those patients eligible for our CPP. Risk stratification will inform patients and clinicians of the likely risk of major complications or death for a group of patients with a similar risk profile undergoing the proposed operation. Clearly, there is no scoring system that perfectly will predict the specific outcome for every individual patient. Risk-adjusted outcome prediction is also important for quality monitoring, measuring actual versus predicted mortality in an institution and allows the assessment of the clinical surgical and anaesthesia performance while adjusting for the risk profile of the patients. Without risk stratification, surgeons and hospitals treating high-risk patients will appear to have worse results than others. This may prejudice referral patterns, affect the allocation of resources and even discourage the treatment of high-risk patients. In our University Hospital the Euroscore and the Nice score (Nederlandse Intensive Care Evaluatie) are now routinely applied for risk stratification and quality assurance.

The use of long-acting sedative and analgesic drugs is associated with prolonged intubation times and longer stay in the intensive care unit. Therefore, we evaluated newer short-acting drugs. In Chapter 5, "Remifentanil provides better protection against noxious stimuli during cardiac surgery than alfentanil", new anaesthetic drugs and techniques were studied that could ameliorate the quality of perioperative medical care in these patients. The potential of the new ultra-short acting opioid remifentanil to provide stable anaesthesia with rapid recovery and good postoperative analgesia in comparison with another short-acting opioid alfentanil, using a Total-IntraVenous based Anaesthetic technique (TIVA) with Target Controlled Infusion (TCI) of propofol, was studied. In the remifentanil patients the suppression of haemodynamic responses to noxious stimuli was superior and overall haemodynamic stability was better. Good postoperative analgesia could easily be obtained by the adninistration of a long-acting opioid before cessation of the infusion of remifentanil. There were no differences in the extubation times between the groups. This study showed that the use of short-acting anaesthetic drugs administered to the patient with specific drug - delivery systems, provides stable anaesthesia with a rapid postoperative recovery.

The reduction in inflammatory response after cardiac surgery can reduce morbidity and mortality. The present study was undertaken to investigate whether certain anaesthetic drugs and/or techniques can decrease the systemic inflammatory response syndrome (SIRS) and in this way make the postoperative course more uneventfull and improve outcome. In Chapter 6, "Comparison of the 
modulatory effects of four different fast-track anesthetic techniques on the inflammatory response to cardiac surgery with cardiopulmonary bypass", we investigated this issue. Four patient groups were compared using a total intravenous anaesthetic technique with target controlled infusion (TCI) of propofol and the opioids alfentanil or remifentanil in different doses and with/without the supplementation of the anaesthetic technique with epidural anaesthesia. We measured bactericidal permeability increasing protein (BPI) as an indicator of the polymorphonuclear neutrophil (PMN) response, interleukin-6 (IL-6) as an inducer of the acute phase response, and lipopolysaccharide binding protein (LBP) and C-reactive protein (CRP) as parameters of the acute phase response during anesthesia and during the first 18 hours postoperatively. A similar pattern in changes of the plasma levels of the measured inflammatory mediators at the different time points was observed in the four experimental patient groups. The significant higher plasma levels of IL-6 in the epidural group at all timepoints as compared to the three general anaesthetic groups was a surprising finding. A possible explanation could be the less intense sympatholytic effect of remifentanil in the epidural group, or a central effect of morphine from the cervical epidural space, where morphine by central autonomic activation of the adrenal cortex can increase IL-6 levels. A third explanation could be that remifenanil itself may decrease the plasma IL-6 concentration. Recent evidence suggests that epidural anaesthesia can potentially affect the inflammatory response to surgery, for example, it can attenuate the postsurgical lymphocyte depression, which is important in infection resistance. The clinical implications of this difference in IL-6 response remains uncertain. The application of routine epidural analgesia in cardiac surgery however remains very controversial, as this technique is associated with the potential formation of epidural hematomas in heparinized patients. This study showed that anaesthetic drugs can modulate and attenuate the inflammatory response to cardiac surgery and therefore can affect outcome.

New surgical techniques include off-pump cardiac surgery. Surgery without cardiopulmonary bypass (CPB) may reduce costs, improve outcome and reduce length-of-stay in ICU and in-hospital. We hypothesized a beneficial effect of offpump surgery on pulmonary outcome after cardiac surgery. This study is described in Chapter 7, "Pulmonary function and inflammatory markers in patients undergoing coronary revascularization with or without cardiopulmonary bypass". We compared a group of coronary surgery patients undergoing surgery with CPB with an off-pump group. The hypothesis was that avoiding cardiopulmonary bypass would result in less inflammation and less organ damage. The conventional cardiac surgery with the use of $C P B$, as compared to the off-pump surgery group, caused an increased systemic inflammatory response as evidenced by the elevated pro-inflammatory cytokines TNF- $\alpha$ and IL-8, not resulting however in a more pronounced pulmonary inflammatory response. The 
increased systemic and bronchial cytokine response did not result in an increase in extra vascular lung water (EVLW) or change in oxygenation ratio. We conclude that off-pump surgery produces no beneficial effect on pulmonary function, as measured with the oxygenation ratio and amount of extravascular lung water. $A$ potential deterioration in pulmonary gas exchange after cardiac surgery is therefore more likely a consequence of the anesthetic, surgical and ICU techniques used which lead to atelectases, rather than an effect of cardiopulmonary bypass. This study emphasizes the importance of preventing and treating intraoperative and postoperative atelectases, mainly by the use of a lung-protective ventilatory strategy.

Over the past 30 years the pulmonary artery catheter (PAC) has become a widely used haemodynamic monitoring device in the management of the cardiac surgical patient. Doubts about its safety and cost-effectiveness have dimished the widespread application of PACs. ${ }^{1,2}$ However, to titrate haemodynamic performance in the vulnerable cardiac surgical patients, cardiovascular monitoring remains of vital importance. We therefore compared the effectiveness of an arterial pulse contour monitor to measure cardiac output and to predict fluid responsiveness with the PAC in Chapter 8, "Assessment of cardiac responsiveness to fluid therapy after cardiac surgery". The main issue here is whether these less invasive and cheaper systems can be used to accurately monitor the cardiac surgical patients for whom this CPP is developed. We investigated whether the new dynamic parameter Stroke Volume Variation (SVV) better predicts the cardiac responsiveness to fluid therapy after cardiac surgery as compared to the old static parameters central venous pressure (CVP) and pulmonary capillary wedge pressure (PCWP), measured with a PAC. The less invasive technique of pulse contour analysis for monitoring continuous cardiac output enables real-time quantification of stroke volume and SVV as dynamic preload variables. The area under the curve, from the receiver operating characteristic curves, for baseline values of SVV was significantly higher than random guess (area $=0.65, \mathrm{p}<0.05$ ), indicative of the value of SVV as a predictor of cardiac responsiveness to fluid therapy. The static preload parameters CVP and PCWP had no predictive value. Monitoring with pulse contour is therefore a very good alternative for PAC in these patients. SVV is even far more superior to the static parameters CVP and PCWP in predicting and for on-line monitoring of cardiac responsiveness to fluid therapy. This study showed that the less-expensive, less-invasive pulse-contour technique is a good alternative for the PAC in these patients.

In Chapter 9, "Shortening ICU stay after coronary artery surgery" we investigated the safety and cost-effectiveness of our Clinical Pathway Protocol. In a randomized clinical trial including 600 patients, we investigated whether our CPP including early postoperative extubation, rapid discharge within 8 hours from the ICU to a step-down unit and early hospital discharge, was safe and cost-effective. 
Patients were randomly assigned to undergo either short-stay intensive care (SSIC) treatment ( 8 hours of intensive care treatment) or control treatment (care as usual, overnight intensive care treatment). The primary outcome variables IC readmission (1.34\% control group and $2.68 \%$ SSIC group) and total hospital stay (8.5 days control group and 8.1 day SSIC group) were not significantly different between the two groups. No significant differences were found between the two groups with respect to postoperative morbidity and 30-day mortality. The SSIC group's quality of life improved more compared with the control group's quality of life. The total hospital costs for the SSIC ( $€ 4.625$ ) were significantly lower as compared to the control group ( $€ 5.441)$ with a mean difference of $-€ 816$. Retrospectively, the charts of the 137 patients which could not be discharged from the ICU within eight hours were analyzed. There were three categories of patients who were not successfully discharged to a step-down unit: (1) weaning from the ventilator was not initiated in time because of inter-current problems ( 55 patients; ischemia, low cardiac out put, excessive bleeding); (2) weaning from the ventilator was initiated in time but rapid weaning failed (65 patients;pulmonary factors, decreased arousal, restless agitation); and (3) rapid discharge failed due to logistic reasons (18 patients; laboratory data delay, human errors concerning the protocol). In retrospect, out of the 137 patients who failed to be discharged within 8 hours after arrival in the ICU to a step down unit, 38 more patients could have been successfully discharged. In this study, recommendations are made how to further improve the success rate of this safe and cost-effective short-stay ICU treatment by the continuous further development of a Clinical Pathway Protocol and by guidance of this protocol by a Continuous Quality Improvement Team. 


\section{DISCUSSION}

The organization and orchestration of a clinical pathway in a hospital is not an easy task to fulfill. It implies change. Change in a big organization is not easy, nothing that is big can be changed easily. Even more difficult is to change something that has proven itself in time, in a place where tradition rules strong and safety burdens progress. There are many layers of management to convince, many different opinions, responsibilities, and interests. All participants involved in the CPP have to face the same direction and work towards the same goal.

There are many different schemes and plans to develop and implement a clinical pathway program: ${ }^{3}$

\section{1) Assessment of the present situation}

A strategic plan has to be formed in perspective of the objectives or the new goals. Therefore, an infrastructure, resources and direction for the program have to be setup, together with an evaluation program. A multi-disciplinary steering committee has to be appointed and a new philosophy has to be adhered to. Important issues have to be discussed: Are all patients potential candidates for fast-track cardiac surgery or only selected patients? ${ }^{4}$ How to define the post-cardiac surgical ICU-model, the step-down units and the graded levels of care? ${ }^{5}$ Motivation of the professionals involved is a priority, with equal and graded levels of responsibilities and with management - and leadership styles in perspective of a learning-environment.

2) The design of a plan

A multi-disciplinary team has to provide multi-modal plans, programs, protocols for best clinical practice based on evidence - based principles. A risk-stratification model and an evaluation model has to be chosen. Research has to become part of the program to find answers to old or new questions. A patient information and education plan, in perspective of patient's expectations, demands and whishes, has to be developed. A training, education and evaluation plan has to be provided for all personnel involved. A documentation and monitoring system has to be installed. Central or key players in the logistics and on the floor have to be appointed. The anaesthesiologist, when involved in the entire perioperative setting, is a pivotal player. Its is very important to understand that the whole effort is a team effort, where communication and motivation of all involved make the program.

\section{3) A pilot implementation}

A test run to find ways of improving the pathway, to ensure maximal usage and acceptance is warranted. There are many obstacles to the implementation of the project to be expected, for example, lack of clinical support and acceptance, anxiety and scepticism among many of the professionals involved. Legal issues, documentation problems, lack of information systems are all essential supporting issues 
4) Full implementation

This phase involves implementation, monitoring and problem solving.

5) The evaluation and integration phase

The predetermined goals and objectives on safety, quality of the care process, complication registration, means and costs evaluation, have to be evaluated in order to constantly improve the implementation process. One has to make sure that the pathway maintains a learning, constantly adapting and flexible program to adjust to the demands of the moment and near future. Data collection, complication registration, evaluation and feedback has to steer the program in the right direction and can be used as a quality instrument. Decisions have to be made who has access to all this new information. ${ }^{6}$

Future directions in the field of fast tracking clinical pathway protocols The world literature on fast tracking cardiac surgical programs mainly investigated selected low risk patient populations. Studies addressing the same issues in high-risk groups are lacking. ${ }^{7}$ However, there is clearly a trend towards fast-tracking the higher-risk patients. ${ }^{8}$ It is generally accepted by clinicians that these patients are also better off with rapid extubation, with shorter ICU stay and with early mobilization. Future research has to verify this.

Some authors propagate the postoperative extubation in the operation room." It We believe that the optimal extubation timepoint, the optimal window of opportunity, lies between two to four hours after arrival in the ICU. ${ }^{15,16}$ Research indicates that in these patients especially the first postoperative hours are characterized by hemodynamic instability, temperature dysregulation, increased mediastinal blood loss and other homeostatic disturbances. Patients can rapidly deteriorate during these first few hours in the ICU, and we believe that these problems are more easy to manage in sedated and ventilated patients.

In some institutions, patients are operated under regional anaesthetic blocks while awake and in some other institutions patients are discharged from the hospital on the first postoperative day. ${ }^{17-23}$ We do not believe these programs provide the best of care. But, in order to provide quality of care and patient safety in a cost-effective manner, searching for borders comes at a price. ${ }^{24-27}$ How far, how fast, should we go or how far should we push the limit is a question time will provide an answer. 


\section{CONCLUSIONS}

In order to be able to describe a Clinical Pathway Protocol (CPP) defining the best clinical practice for low - risk coronary artery surgery patients, based on the principles of evidence-based medicine and taking into account economical aspects, six studies had to be performed. All six studies provided invaluable information in the description of our CPP that incorporated fast extubation, short-stay ICU, rapid mobilization and early hospital discharge.

Study 1 . The use of a risk stratification system is necessary for proper decision making in implementing fast-tracking and short-stay intensive care, and is essential for quality assessment.

Study 2. Anaesthesia management should focus on short-acting drugs. Remifentanil provides better protection against noxious stimuli than alfentanil and makes early extubation possible.

Study 3. Anaesthetic drugs can modulate the inflammatory response syndrome after surgery and in this way improve outcome; optimal analgesia management is imperative in fast-track cardiac programs. Supplementation of a fast-track anaesthetic technique with epidural analgesia preserves haemodynamic stability and is associated with faster extubation times and less postoperative pain.

Study 4. Intraoperative and postoperative care should focus on the prevention of pulmonary atelectases by implementing a lung-protective ventilation protocol. Surgery without the use of cardiopulmonary bypass does not result in less lung dysfunction as measured by inflammatory cytokines, amount of extra vascular lung water, and oxygenation ratios as compared to the conventional technique with cardiopulmonary bypass.

Study 5. Perioperative cardiovascular care can be titrated with less-invasive cardiovascular pulse - contour monitoring devices and is cost-effective. Stroke volume variation is a better parameter for the assessment of cardiac responsiveness to fluid therapy after cardiac surgery than the older static parameters central venous pressure and pulmonary capillary wedge pressure.

Study 6. We introduced short stay intensive care (SSIC) for low-risk coronary artery surgery patients. In a large randomized trial in 600 patients we demonstrated that our protocol was safe and cost-effective. 


\section{REFERENC:ES}

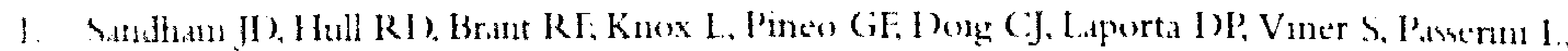
1) R.andunzed. Cintrolled Irial of the Use of l'ulmonury-Artery Catheters in High-Risk Surgh al

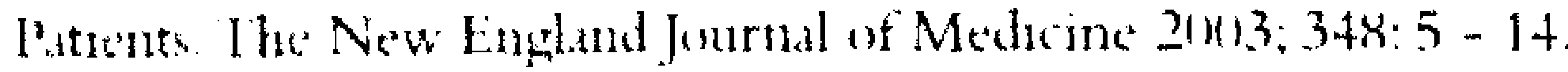

2. Harvey S, Harrison I)A. Singer M. Asheroft J. Jones (M. Elbourne I), Bramton W. Willum 1)

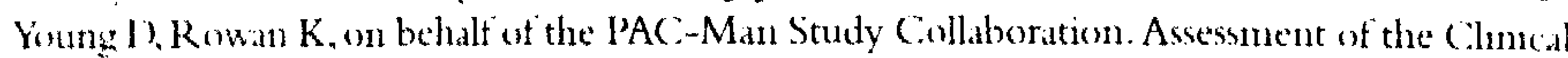

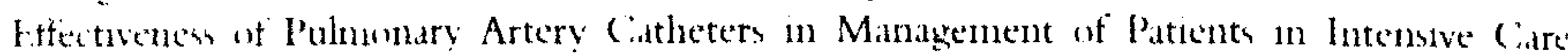
(PAC $-M a n)$ : A Randomised Controlled Trial. The Lancet 2015; 366: $472-477$.

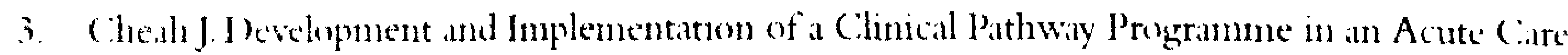
(reneral Humpital in Singapore. International Journal for Quality in Health Care 2(14): 12: $4113-$ 412.

4. Royatum I. Pastent Selectum and Anestletic Management for Early Extubation and Hosptal

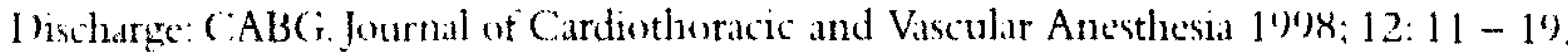

5. Wake ly and (heng I CH. Postoperative Intensive Care in Cardiac Surgery. Current Opmon m Andesthexiulogy $20101 ; 14:+1-45$.

6. White SM. Confidentiality" No Blame Culture" and Whistleblowing Non-Physician Practice and Accountability: Best Practice \& Revearch Clinical Anaesthesiology 2(1)6;21):525 - 543

7. Allan C., Tordman Fi Karabulut EH, Tarcan S, Dagdelen S, Eren N, and Calglar N. Fast Track Recovery of High Risk Coronary Bypass Surgery Patients. European Journal of CardioThoracit Surgery $2(13 ; 23: 678-683$.

x. London MJ, Shrover ALW, and Grover Fl. Fast Tracking into the New Millemium. An Evolving Paradigm. Anesthesiology 1949:91:911-915

9. Royse CF Ruyse AG, and Soeding PF Routine Immediate Extubation After Cardiac Operation A Review of Our First lin Patients. Annals of Thoracic Surgery 1999;68:1326-132\%.

10. Lee TWR and Jacobsohn E. Pro: Tracheal Extubation Should Occur Routinely in the Operating Room After Cardiac Surgery. Journal of Cardiothoracic and Vascular Anesthesia 20101; $14: 6113$ 610.

11. Peragallo RA and Cheng DCH. Con: Tracheal Extubation Should Not Occur Routinely in the Operating Room After Cardiac Surgery. Journal of Cardiothoracic and Vascular Anesthesia 2mk:; $14: 611-613$

12. Djaiani GN. Ali M, Heinnich L. Bruce J, Carroll J, Karski J, Cusimano RJ, and Cheng DCH Ultra-Fast-Track Anesthetic Technique Facilitates Operating Room Extubation in Patients Undergoing Off-Pump Coronary Revascularization Surgery. Journal of Cardiothoracic and Vascular Anesthesia 2001; 15:152 - 157.

13. Straka Z, Brucek P, Vanek T, Votava J, and Widimsky P. Routine Immediate Extubation for OffPump Coronary Artery Bypass Grafting Without Thoracic Epidural Analgesia. Annals of Thoracic Surgery 20(12;74:1544-1547.

14. Horswell JL, Herbert MA, Prince SL, and Mack MJ. Routine Immediate Extubation After OtfPump Coronary Artery Bypass Surgery: 514 Consecutive Patients.Journal of Cardiothoracic and Vascular Anesthesia 20105; 19: $282-287$.

15. Higgins T. Safety lssues Regarding Early Extubation After Coronary Artery Bypass Surgery. Journal of Cardiothoracic and Vascular Anesthesia 1995:9:24-29.

16. Mangano DT. Biventricular Function After Myocardial Revascularization in Humans: Deteriord tion and Recovery Patterns During the First 24 Hours. Anesthesiology 1985; 62:571 - 577.

17. Karagoz IIY, Sonnez B, Bakkaloglu B, Kurtoglu M. Erdinc M, Turkeli A, and Bayazit K. Coronary Artery Bypass Crufting in the Conscious patient Without Endotracheal General Anesthesta Anth of Thoracic Surgery 20101; 70:91 - 96. 
18. Anderson MB, Kwong KF, Furst AJ, and Salerno TA. Thoracic Epidural Anesthesia for Coronary Bypass via Left Anterior Thoracotomy in the Conscious Patient. European Journal of CardioThoracic Surgery 2001;20:415-417.

19. Vanek T, Straka Z, Brucek P, and Widimsky P. Thoracic Epidural Anesthesia for Off-Pump Coronary Artery Bypass Without Intubation. European Journal of Cardio-Thoracic Surgery $2001 ; 20: 858-860$.

20. Chakravarthy M, Jawali V, Patil TA, Jayaprakash K, and Shivananda NV. High Thoracic Epidural Anesthesia as the Sole Anesthetic for Performing Multiple Grafts in Off-Pump Coronary Artery Bypass Surgery. Journal of Cardiothoracic and Vascular Anesthesia 2003; 17: 160 - 164.

21. Aybek T, Kessler P, Khan MF, Dogan S, Neidhart G, Moritz A, and Wimmer-Greinecker G. Operative Techniques in Awake Coronary Artery Bypass Grafting. The Journal of Thoracic and Cardiovascular Surgery 2003; 125: $1394-1404$.

22. Chakravarthy M, Jawali V, Patil TA, Jayaprakash K, Kolar S, Joseph G, Das JK, Maheswari U, and Sudhakar N. Conscious Cardiac Surgery With Cardiopulmonary Bypass Using Thoracic Epidural anesthesia Without Endotracheal General Anesthesia. Journal of Cardiothoracic and Vascular Anesthesia 2005; 19:300 - 305.

23. Calafiore AM, Scipioni G, Teodori G, Di Giammarco G, Di Mauro M, Canosa C, Iaco AL, and Vitolla $G$. Day 0 Intensive Care Unit Discharge - Risk or Benefit for the Patient who Undergoes Myocardial Revascularization? European Journal of Cardio-Thoracic Surgery 2002; $21: 377$ 384.

24. Lahey SJ, Campos CT, Jennings B, Pawlow P, Stokes T, and Levitsky S.Hospital Readmission After Cardiac Surgery. Does 'Fast-track' Cardiac Surgery Result in Cost Saving or Cost Shifting? Circulation 1998; 98:1I-35-II40.

25. Baisden CE, Bolton JWR, and Riggs MW. Readmission and Mortality in Patients Discharged the Day After Off-Pump Coronary Bypass surgery. Annals of Thoracic Surgery 2003; 75: 68 73.

26. Mora Mangano CT. Risky Business. The Journal of Thoracic and Cardiovascular Surgery 2003; 125: $1204-1207$.

27. Cheng DCH. Routine Immediate Extubation in the Operating Room After OPCAB Surgery: Benefits for Patients, Practitioners, or Providers? Journal of Cardiothoracic and Vascular Anesthesia $2005 ; 19: 279-281$. 
CHAPTER 11

Samenvatting, Discussie en Conclusies 


\section{SAMENVATTING}

In dit proefschrift wordt de ontwikkeling beschreven van een Klinisch Zorglijn Protocol (Clinical Pathway Protocol) voor patiënten die kransslagaderchirurgie ondergaan in het Academisch Ziekenhuis Maastricht. Het doel was het opstellen van een protocol met de beste mogelijke zorg ('best clinical practice') voor deze patiënten, gebaseerd op de principes van wetenschappelijk aantoonbaar effect (evidence-based medicine) en rekening houdend met economische aspecten van klinische zorg. Bijzondere nadruk werd gelegd op de anesthesiologische zorg rondom een hartoperatie en het optimale gebruik van de in het ziekenhuis beschikbare faciliteiten zoals de intensieve zorg afdeling en de lagere-zorg afdelingen na de hartoperatie. De medische zorg volgens het Klinisch Zorglijn Protocol omvatte snelle postoperatieve extubatie, het binnen 8 uur ontslaan van de patiënten van de intensieve zorg afdeling naar een afdeling met een lager nivo van zorg en snel ontslag uit het ziekenhuis. Tijdens het opstellen van dit protocol zijn verschillende vragen naar boven gekomen waarvoor in de bestaande literatuur geen kant en klare antwoorden gevonden konden worden. Deze onbeantwoorde vragen hebben geleid tot de formulering van zes onderzoeksvragen. Vervolgens zijn zes studies uitgevoerd om een Klinisch Zorglijn Protocol te kunnen schrijven dat kosten-effectief is, en gebaseerd op evidence-based 'best clinical practice' voor de geselecteerde patiëntengroep. Deze zes studies worden in dit proefschrift beschreven.

In Hoofdstuk 2, "Geschiedenis van de medische zorg rondom een hartoperatie" wordt een kort overzicht gegeven van de ontwikkeling van de anesthesiologische zorg in de hartchirurgie vanaf de vroege vijftiger jaren tot heden. In de loop van de tijd is de vraag vanuit de matschappij naar medische zorg en mogelijkheden alleen maar toegenomen, aan de andere kant is er ook een steeds maar toenemend aanbod van medische ontwikkelingen en mogelijkheden. Deze factoren tezamen, en de steeds ouder wordende bevolking, leggen een haast onacceptabel hoge financiële druk op de schouders van medische zorginstellingen en hun regeringen. De regeringen moeten zowel de kosten als bronnen beheersen. Economische factoren zijn noodzakelijkerwijs onderdeel van het medisch beslissingsproces geworden, een proces dat voorheen enkel toebehoorde aan de medische specialisten. Het gevolg hiervan is dat de medische wereld de manier waarop patiënten worden behandeld moet veranderen en zich moet aanpassen aan de nieuwe vragen en verwachtingen van de bevolking en de regering. Deze taak is verre van gemakkelijk, de medische wereld moet meer en beter doen met minder. Een nieuw concept 'Evidence Based Medicine' is geïntroduceerd met als doel om de medische praktijkvoering te baseren op wetenschappelijk aantoonbare bevindingen, om subjectief medisch handelen te vermijden zodat kosten-baten ratios kunnen stijgen. De combinatie van Evidence Based Medicine met klinische exper- 
tise en patiëntenwaarden is vervolgens vertaald in richtlijnen en protocollen voor het ziekenhuis. De medische gedachte hoe patiënten het beste behandeld kunnen worden is veranderd van een medisch specialist georiënteerde medische zorg naar een patiënten georiënteerde medische zorg. Een gevolg hiervan is het ontstaan van zogenaamde Klinische Zorglijnen. In dit proefschrift wordt de ontwikkeling van een Klinisch Zorglijn Protocol voor electieve laag-risico kransslagaderoperatie patiënten beschreven.

Het doel was om een Klinisch Zorglijn Protocol te schrijven voor laag-risico kransslagaderoperatie patiënten. Hiertoe was het noodzakelijk om patiënten te kumnen onderverdelen in laag risico en hoog risico patiënten. In Hoofdstuk 4, "Risico inschatting en ongewenste medische resultaten na hartchirurgie", hebben we de literatuur onderzocht naar risico-inschatting en risicoevaluatie systemen die ons zouden kunnen helpen bij het selecteren van patiënten die in aanmerking komen voor ons Klinisch Zorglijn Protocol. Risico inschatting zal patiënten en clinici informeren over het mogelijk risico van eventuele complicaties en overlijden voor een groep patiënten met een vergelijkbaar risicoprofiel die de voorgestelde operatie moeten ondergaan. Uiteraard bestaat er geen risicoinschattingsysteem dat perfect de specifieke uitkomst zal voorspellen voor iedere individuele patiënt. Risico-gerelateerd resultaat voorspellen is eveneens belangrijk voor het bewaken van kwaliteit en voor het meten van actuele versus voorspelde mortaliteit binnen een instelling. Hiermee kunnen de chirurgische en anesthesiologische prestaties bekeken worden terwijl aanpassingen worden gemaakt voor het risicoprofiel van de patiënten. Zonder risicoinschatting, lijken chirurgen en ziekenhuizen die hoog-risico patiënten behandelen, slechtere resultaten te behalen dan anderen. Hierdoor zouden voorkeur-verwijzingspatronen kunnen ontstaan, de toekenning van middelen kunnen beVnvloed worden en zelfs de behandeling van hoog- risico patiënten ontmoedigd. In ons universiteits ziekenhuis worden de Euroscore en de Nice score (Nederlandse Intensive Care Evaluatie) nu als routine toegepast voor risico-inschatting en kwaliteitgarantie.

Het gebruik van langwerkende slaapmiddelen en pijnstillers wordt geassocieerd met langere tijden die patiënten moeten doorbrengen aan een beademingsmachine en een langer verblijf binnen de intensieve-zorg afdeling. We hebben dan ook nieuwere, kortwerkende medicijnen onderzocht. In Hoofdstuk 5, "Remifentanil geeft betere bescherming tegen schadelijke stimuli gedurende hartchirurgie dan Alfentanil", hebben we nieuwe anesthesiologische medicijnen en technieken bestudeerd die de kwaliteit van de medische zorg rond een operatie bij deze patiënten kunnen verbeteren. De mogelijkheden van het nieuwe ultrakortwerkende opium-achtige middel remifentanil, met betrekking tot het verschaffen van een stabiele anesthesie, snel herstel na operatie en goede postoperatieve pijnstilling, in vergelijking tot een ander kortwerkend opiaat alfentanil, gebruik makend 
van een volledig intraveneuze anesthesie techniek (TIVA) met plasmaconcentratiegecontroleerde infusie (Target Controlled Infusion) van propofol, werd bestudeerd. Bij de remifentanil patiënten was de onderdrukking van de schadelijke stimuli en stabiliteit van hartfrequentie en bloeddruk beter. Goede postoperatieve pijnstilling kon gemakkelijk bereikt worden door de toediening van een langer werkend opiaat voor de beëindiging van de remifentanil infusie. Er waren geen verschillen in de extubatietijden tussen de groepen patiënten. Dit onderzoek toonde aan dat het gebruik van kortwerkende anesthesiologische medicijnen, aan patiënten gegeven middels een specifiek toedieningssysteem, stabiele anesthesie met een snel herstel na de operatie geeft.

Het verminderen van de ontstekingsreactie na hartchirurgie kan de kans op ziekte en sterfte verlagen. Het onderhavig onderzoek is uitgevoerd om te bekijken of bepaalde anesthesiologische medicijnen en/of technieken de ontstekingsreactie (SIRS) die optreedt na de operatie kunnen verminderen om zo het klinisch verloop na de hartoperatie te optimaliseren met als uiteindelijk doel betere resultaten te behalen. In Hoofdstuk 6 "Vergelijking van de modulerende effecten van vier verschillende anesthesietechnieken op de ontstekingsreactie na hartchirurgie met hart-long machine", hebben we dit onderwerp onderzocht. Vier patiëntengroepen werden vergeleken, gebruik makend van een volledig intraveneuze anesthesietechniek (TIVA) met plasmaconcentratie-gecontroleerde infusie (TCI) van propofol en de opioiden alfentanil of remifentanil in verschillende doseringen alsook een remifentanil-groep in combinatie met een hoge epidurale anesthesietechniek. We hebben bactericidal permeability increasing protein (BPI) bekeken als een indicator van de neutrofiele granulocyt (polymorphonuclear neutrophil) reactie, interleukin-6 (IL-6) als een aanzetter van de acute fase reactie, en lipopolysaccharide binding protein (LBP) en C-reactive protein (CRP) als parameters van de acute fase reactie gedurende anesthesie en gedurende de eerste 18 uur postoperatief. Er werd een vergelijkbaar patroon in veranderingen van de plasmaniveaus van de gemeten ontstekingsmediatoren op de verschillende tijdstippen geobserveerd in de vier experimentele patiëntengroepen. Het significant hoger plasma niveau van IL-6 in de epidurale groep op alle tijdstippen vergeleken met de drie algemene anesthesie groepen was een verbazingwekkende bevinding. Een mogelijke verklaring zou het minder intense sympathicolitische effect van remifentanil kunnen zijn in de epidurale groep, of een centraal effect van morfine vanuit de cervicale epidurale ruimte, waar morfine door centrale autonome activatie van de bijnierschors IL- 6 concentraties kan doen toenemen. Een derde verklaring zou kunnen zijn dat remifenanil zelf de plasma IL-6 concentratie zou kunnen laten afnemen. Uit recent onderzoek blijkt dat epidurale anesthesie de ontstekingsreactie die volgt op de chirurgische prikkel zou kunnen beVnvloeden, het kan de postchirurgische lymphocyten depressie verminderen, die belangrijk is voor infectie resistentie. De klinische implicatie van het verschil in IL-6 reactie 
blijft een raadsel. De routine toepassing van epidurale pijnstilling in hartchirurgie blijft echter controversieel, daar deze techniek geassocieerd wordt met de potentiële vorming van een z.g. epidurale bloeduitstorting (hematoom) speciaal in ontstolde (geheparinizeerde) patiënten. Dit onderzoek heeft aangetoond dat anesthesiologische medicijnen de ontstekingsreactie na een hartoperatie kunnen beVnvloeden en verzwakken en dientengevolge de uiteindelijke resultaten van een hartoperatie kunnen beVnvloeden.

Nieuwe hartoperatietechnieken omvatten het opereren van kransslagaders zonder gebruikmaking van de hart-long machine (off-pump hartchirurgie). Hartoperaties zonder gebruikmaking van de hart-long machine kunnen kostenbesparend zijn, betere resultaten opleveren en het verblijf op de intensieve zorg afdeling en in het ziekennuis verkorten. We veronderstelden een gunstig effect van offpump hartchirurgie op de longfunctie na hartchirurgie. Dit onderzoek wordt beschreven in Hoofdstuk 7, "Longfunctie en ontstekingsindicatoren bij patiënten die kransslagaderchirurgie ondergaan met of zonder gebruik van de hart-long machine". We hebben een groep kransslagaderoperatie-patiënten die een operatie ondergingen met gebruikmaking van een hart-long machine vergeleken met een groep patiënten die zonder gebruikmaking van een hart-long machine (off-pump) geopereerd werden. De hypothese was dat het vermijden van de hart-long machine zou resulteren in minder ontstekingsreacties en minder orgaanschade. De patiëntengroep die geopereerd werd met behulp van de hart-long machine, vergeleken met de patiënten die zonder hart-long machine geopereerd werden, had een verhoogde systemische ontstekingsreactie zoals blijkt uit de verhoogde pro-inflammatoire cytokines TNF- $\alpha$ (tumor necrosis factor alpha) en IL-8 (interleukine-8), hetgeen echter niet resulteerde in een meer uitgesproken pulmonale ontstekingsreactie. De verhoogde systemische en bronchiale cytokine reactie resulteerde niet in een toename van extravasculair longwater (EVLW) of verandering in oxygenatie ratio. We concluderen dat kransslagaderchirurgie zonder hart-long machine geen voordelig effect heeft op de longfunctie, zoals gemeten met de oxygenatie ratio en hoeveelheid extra vasculair long water. Het is dan ook waarschijnlijker dat een mogelijke verslechtering van pulmonale gasuitwisseling na hartchirurgie een gevolg is van anesthesiologische, chirurgische en intensieve zorg technieken die leiden tot atelectase (huchtarmoede van de longblaasjes), dan een gevolg van de hart-long machine. Dit onderzoek benadrukt het belang van preventie en behandeling van intraoperatieve en postoperatieve atelectase-vorming, voornamelijk door het gebruik van longbeschermende beademingsstrategieën.

Gedurende de laatste 30 jaren is de arteria pulmonalis-catheter een wijdverbreid instrument voor het monitoren van de hart-en bloedvaten-functie geworden van de hartoperatie patiënt. Twijfels over de veiligheid en kosten-effectiviteit 
hebben de wijdverbreide toepassing van arteria pulmonalis-catheter doen dalen. 1.2 Echter, het titreren van het hartdebiet in de kwetsbare hartchirurgische patiënt en het bewaken van hart- en bloedvaten-functie is uiterst belangrijk. We hebben daarvoor de effectiviteit van een middels bloeddrukgolfanalyse-berekend hartdebiet en reactie van het hart op een volume belasting vergeleken met waarden verkregen met de arteria pulmonalis-catheter in Hoofdstuk 8, "Inschatting van het hartdebiet na volume therapie na hartchirurgie". Belangrijk te weten is of deze minder invasieve en goedkopere systemen gebruikt kunnen worden om op accurate wijze de hartchirurgische patiënt, voor wie dit Klinisch Zorglijn Protocol is ontwikkeld, te monitoren. We hebben onderzocht of de nieuwe dynamische parameter slagvolume variatie beter de hartreactie na volume therapie na een hartoperatie voorspelt, vergeleken met de oude statische parameters centraalveneuze druk en wiggedruk, gemeten met een arteria pulmonalis-catheter. De minder invasieve techniek van bloeddrukgolfanalyse om continu het hartdebiet te meten maakt actuele (real time) kwantificatie van slagvolume en slagvolumevariatie mogelijk. Het gebied onder de curve, van de receiver operating characteristic curve, voor uitgangswaarden van slagvolume variatie was significant hoger (gebied onder de curve $=0,65, \mathrm{p}<0,05)$, indicatief voor het belang van slagvolumevariatie als een voorspeller van reactie van het hart na volumetherapie. De statische vullingsparameters (preload) van het hart, centraalveneuze druk en wiggedruk, hadden geen voorspellende waarde. Het monitoren met behulp van de bloedrukgolfanalyse is dan ook een heel goed alternatief voor arteria pulmonalis-catheter bij deze patiënten. Slagvolume variatie scoort een stuk beter in vergelijking tot de statische parameters centraalveneuze druk en wiggedruk in het voorspellen van en on-line monitoren van het hartdebiet na volume therapie. Uit dit onderzoek blijkt dat de goedkopere, minder invasieve bloedrukgolfanalyse techniek een goed alternatief is voor de arteria pulmonalis-catheter bij deze groep patiënten.

In Hoofdstuk 9, "Het verkorten van de verblijfsduur op de intensievezorg afdeling na kransslagaderchirurgie" hebben we de veiligheid en kosteffectiviteit van ons Klinisch Zorglijn Protocol bestudeerd. In een gerandomiseerde klinische trial van 600 patiënten, hebben we bekeken of het Klinisch Zorglijn Protocol, inclusief snelle postoperatieve extubatie, snel ontslag binnen 8 uur van de intensieve-zorg afdeling naar een lagere-zorg afdeling en snel ziekenhuis ontslag, veilig en kosten-effectief was. De patiënten werden volgens een willekeurige steekproefbepaling aangewezen om ofwel korte verblijfsduur intensieve-zorg (ShortStay Intensive Care, SSIC) behandeling te ondergaan ( 8 uur intensieve-zorg behandeling) of standaard behandeling (zorg zoals gebruikelijk, intensieve-zorg behandeling tot de volgende dag). De primaire onderzoeksresultaten, heropname op de intensieve-zorg afdeling (1,34\% controle groep en $2,68 \%$ SSIC groep) en totaal ziekenhuis verblijf ( 8,5 dagen controle groep en 8,1 dagen SSIC groep) waren niet significant verschillend tussen de twee groepen. Er zijn geen significante verschillen 
gevonden tussen de twee groepen met betrekking tot postoperatieve morbiditeit en 30-dagen mortaliteit. De kwaliteit van leven van de SSIC- groep verbeterde meer in vergelijking tot de kwaliteit van leven van de controle groep. De totale ziekenhuis kosten voor de SSIC-groep ( $€ 4$.625) waren significant lager vergeleken met de controle-groep ( $€ 5.441)$ met een gemiddeld verschil van $-€ 816$. Achteraf werden de gegevens geanalyseerd van de 137 patiënten die niet binnen 8 uur van de intensieve-zorg afdeling konden worden ontslagen. Er waren drie categorieën patiënten die niet succesvol werden overgeplaatst naar een lagere-zorg afdeling: (1) het ontwennen van de beademingsmachine was niet op tijd ten gevolge van klinische onregelmatigheden ( 55 patiënten; zuurstoftekort van het hart, te laag hartdebiet, overmatig postoperatief bloedverlies); (2) het ontwennen van de beademingsmachine was tijdig begonnen maar snelle extubatie kon niet plaats vinden ( 65 patiënten; longfunctie factoren, verlaagd bewustzijn, onrust en agitatie); en (3) snel ontslag vond niet plaats om logistieke redenen (18 patiënten; vertraging in laboratoria data, menselijke vergissingen betreffende het protocol). Terugkijkend, van de 137 patiënten die na 8 uur verblijf in de intensieve-zorg afdeling niet konden worden overgebracht naar een lagere-zorg afdeling, hadden 38 patiënten toch succesvol overgebracht kunnen worden. In deze studie zijn aanbevelingen opgesteld ter verbetering van de slagingskans van deze veilige en kosten-effectieve kortverblijf intensieve-zorg (Short Stay Intensive Care) behandelingsmethode door het Klinisch Zorglijn Protocol continu te verbeteren door een Continu Kwaliteits Verbeterings Team.

\section{DISCUSSIE}

Het organiseren en orchestreren van een klinische zorglijn binnen een ziekenhuis is geen gemakkelijke taak. Het betekent verandering, en verandering in een grote organisatie is niet gemakkelijk. Het is zelfs nog moeilijker om iets te veranderen dat zich reeds bewezen heeft binnen een omgeving met een stevige traditie en waar veiligheid vooruitgang bemoeilijkt. Er zijn verschillende management niveaus die overtuigd moeten worden, veel verschillende meningen, verantwoordelijkheden en belangen. Alle betrokkenen binnen de klinisch zorglijn moeten dezelfde kant op kijken en samenwerken voor hetzelfde doel. Er bestaan verschillende schema's en plannen om een klinische zorglijn te ontwikkelen en te implementeren: ${ }^{3}$

\section{1) Beoordeling van de huidige situatie}

Er dient een strategisch plan te worden opgesteld in het licht van de doelstellingen of het nieuwe doel. Hiertoe dient een infrastructuur, bronnen en richting voor het programma te worden opgesteld, tezamen met een evaluatie programma. Een multidisciplinaire stuurgroep dient te worden aangewezen en een nieuwe filosofie dient ontwikkeld te worden. Belangrijke onderwerpen dienen op de agenda te 
worden gezet: zijn alle patiënten potentiële kandidaten voor kortverblijf intensievezorg hartchirurgie (Fast-Track en Short-Stay Intensive Care) of enkel geselecteerde patiënten? ${ }^{+}$Hoe dienen het postoperatief-hartchirurgisch intensief-zorgmodel, de lagere-zorg afdelingen en de verschillende zorgniveaus te worden gedefiniëerd? Het motiveren van de betrokken professionals met dezelfde en verschillende verantwoordelijkheden heeft prioriteit. Management - en leiderschapstijlen dienen in perspectief van een lerende omgeving en organisatie te staan.

\section{2) Het ontwerp van een plan}

Een multidisciplinair team dient zorg te dragen voor multi-modale plannen, programma's en protocollen voor goede klinische zorg ('best clinical practise'), die wetenschappelijk onderbouwd is. Een risicostratificatie model en een evaluatie model dient te worden gekozen. Onderzoek dient onderdeel te worden van het programma om antwoorden te vinden op bestaande en nieuwe vragen. Er dient een patiënten-informatie en - educatie plan te worden ontwikkeld, rekening houdend met de verwachtingen, verzoeken en wensen van patiënten. Er dient een training-, onderwijs- en evaluatie-plan te zijn voor het betrokken personeel. Een documentatie- en monitoring-systeem dient te worden geVnstalleerd. Binnen de logistiek en op de werkvloer dienen er centrale of sleutelfiguren worden aangesteld, de anesthesioloog-intensivist zal hierin een spilfunctie innemen. Het is heel belangrijk te beseffen dat het hele gebeuren een teamgebeuren is en dat het programma staat of valt met de motivatie van eenieder.

\section{3) Een pilot implementatie}

Een testfase kan gebruikt worden om te bekijken of de klinische zorglijn verbeterd kan worden, of het maximaal gebruikt en geaccepteerd wordt. Er zijn vele obstakels voor de implementatie van het project te verwachten, bijvoorbeeld, het gebrek aan klinische ondersteuning en acceptatie, angst en scepticisme onder veel van de betrokkene professionals. Wet en regelgeving, documentatie problemen, gebrek aan informatie systemen, het zijn allemaal essentiële ondersteunende items die opgelost dienen te worden.

\section{4) Volledige implementatie}

Deze fase bestaat uit implementatie, monitoring en het oplossen van problemen.

\section{5) De evaluatie en integratie fase}

De vooraf opgestelde doelen en doelstellingen met betrekking tot veiligheid, kwaliteit van het zorgproces, complicatie registratie, middelen en kosten, dienen te worden geëvalueerd om steeds weer het implementatie proces te verbeteren. Men dient er voor te zorgen dat het klinisch zorgtraject een lerende organisatie blijft die flexibel is en zich constant aanpast aan nieuwe omstandigheden, niet alleen aan de eisen van vandaag maar ook aan die voor de toekomst. Data verzameling, complicatie registratie, evaluatie en feedback dienen het programma in de juiste richting te 
sturen en kunnen gebruikt worden als kwaliteitsinstrumenten. Er moet vooraf besloten worden wie toegang heeft tot al deze nieuwe en mogelijk verwarrende informatie."

Toekomstige ontwikkelingen in de wereld van snelle extubatie en klinische zorglijn protocollen.

Wetenschappelijk onderzoek betreffende snelle extubatie na hartchirurgie (FastTrack-Anesthesia) betreft met name laag-risico patiëntenpopulaties. Studies over dezelfde onderwerpen binnen de hoog-risico groepen ontbreekt veelal. ${ }^{7}$ Echter, er is een duidelijke trend in de richting van snelle extubatie van hoog-risico patiënten. ${ }^{8}$ Het is algemeen geaccepteerd dat deze patiënten ook beter af zijn met een snelle extubatie, een korter verblijf op de intensieve-zorg afdeling en met snellere mobilisatie. Toekomstig onderzoek zal dit moeten uitwijzen.

Sommige auteurs zijn voorstander van extubatie na de hartoperatie in de operatiekamer." ${ }^{\text {"1t }}$ We zijn van mening dat het optimale tijdstip voor extubatie ligt tussen de twee tot vier uur na aankomst op de intensieve-zorg afdeling. ${ }^{15.11}$ Uit onderzoek blijkt dat deze patiënten, met name de eerste postoperatieve uren, gekenmerkt worden door instabiliteit van de hartfunctie, hartfrequentie en bloedvatenfunctie, temperatuur ontregelingen, verhoogd bloedverlies uit het operatiegebied en andere homeostatische verstoringen. Patiënten kunnen in deze eerste uren in snel tempo achteruitgaan en we zijn van mening dat deze problemen beter kunnen worden aangepakt bij gesedeerde en beademde patiënten.

In sommige instellingen worden patiënten geopereerd met gebruikmaking van locale analgesie technieken (thoracale epiduraal) terwijl ze licht gesedeerd zijn, in weer andere instellingen worden patiënten op de eerste postoperatieve dag ontslagen uit het ziekenhuis. ${ }^{17-23}$ We denken niet dat deze programma's de beste mogelijke zorg geven. Maar, om kwaliteit en veiligheid te leveren op een kosteneffectieve manier, gaat het opzoeken van grenzen ten koste van een prijs. ${ }^{2+-27}$ Hoe ver of hoe snel we moeten gaan of hoe ver we de grenzen moeten opzoeken, dat is een vraag die de tijd ons zal leren.

\section{CONCLUSIES}

Voor de ontwikkeling van een Klinisch Zorglijn Protocol, het definiëren van wat wordt beschouwd 'Best Clinical Practice' (de beste medische zorg die mogelijk is), voor laag risico hartoperatiepatiënten, gebaseerd op de principes van EvidenceBased Medicine (wetenschappelijk aantoonbaar resultaat) en rekening houdend met economische aspecten, zijn zes studies uitgevoerd. Alle zes studies hebben belangrijke informatie opgeleverd voor de totstandkoming van ons Klinisch Zorglijn Protocol dat snelle extubatie (fast-track anesthesia), kort-verblijf intensievezorg (short-stay intensive care), snelle mobilisatie en snel ziekenhuis ontslag omvatte. 
Studie 1. Het gebruik van een risico-stratificatie systeem is noodzakelijk om de juiste klinische beslissingen te kunnen nemen bij het implementeren van vroege extubatie en kortverblijf intensieve-zorg en is essentieel voor kwaliteitsbeoordeling.

Studic 2. Anesthesiologisch management dient de nadruk te leggen op kortwerkende medicijnen. Remifentanil beschermt beter tegen schadelijke stimuli dan alfentanil en maakt snelle extubatie mogelijk.

Studic 3. Anesthesiologische middelen kunnen de ontstekingreactie (SIRS) na hartchirurgie beïnvloeden en zo de resultaten van een operatie verbeteren; optimale postoperatieve pijnstilling is een must voor hartoperatieprogramma's die vroege extubatie nastreven. De combinatie van algehele anesthesie met een hoge epidurale techniek zorgt voor een stabiel verloop van hartfrequentie en bloeddruk, maakt snelle extubatie mogelijk en zorgt voor optimale postoperatieve pijnstilling.

Studie 4. Bij intraoperatieve- en postoperatieve zorg dient nadruk te liggen op het voorkomen van atelectasevorming door het implementeren van een longbeschermend beadmingsprotocol. Hartchirurgie zonder gebruik te maken van de hart-long machine (off-pump) resulteert niet in minder longdysfunctie gemeten aan de hand van de ontstekingsreactie, hoeveelheid extravasculair longwater, en oxygenatie ratios in vergelijking tot de conventionele hartoperatie met de hartlong machine.

Studie 5. Perioperatieve hart- en bloeddruk variaties kunnen nauwkeurig worden bewaakt met behulp van minimaal invasieve bloeddrukgolfanalyse technieken en deze zijn kosten-effectief. Slagvolume variatie is een betere parameter voor de beoordeling van het hartdebiet na een volumebelasting na hartchirurgie dan de oudere statische parameters centraal veneuze druk en wiggedruk.

Studie 6. We hebben kort verblijf intensive-zorg (Short-Stay Intensive Care) geVntroduceerd voor laag-risico kransslagader-operatiepatiënten. In een grote gerandomiseerde studie van 600 patiënten hebben we aangetoond dat ons protocol veilig en kosteffectief was. 


\section{DANKWOORD}

De realisatie van een proefschrift vergt de inzet van velen. De totstandkoming van dit proefschrift des te meer, daar niet alleen op velen een beroep werd gedaan voor het uitvoeren van de klinische studies, maar ook voor het ontwikkelen, plannen, implementeren en evalueren van een nieuw zorgtraject binnen het HartVaatcentrum van het academisch ziekenhuis Maastricht, het "Short-Stay Intensive Care after Cardiac Surgey" project.

Allereerst wil ik diegene bedanken die de meeste tijd en energie in de totstandkoming van dit proefschrift heeft gestoken, mijn onderzoeksbegeleider en copromotor Dr. Roekaerts. Ook zijn echtgenote Monika dank ik voor de vele uren vrije tijd die Paul aan dit proefschrift kon besteden. Beste Paul, jouw inzichten en conclusies bleven en blijven mij telkens weer verbazen en daardoor aanzetten om ook deze vaardigheden meester te worden. Ik heb je leren kennen als een serieus, intelligent, hard werkend persoon en dokter, iemand voor wie de patiënt centraal staat, met een gewogen mening puttend uit jarenlange klinische en wetenschappelijke ervaring, en die op een bescheiden manier als voorbeeld voor anderen wil dienen. Door onze nauwe samenwerking heb ik heel veel van je mogen leren, niet alleen op klinisch en onderzoeksgebied, maar ook daarbuiten. Ik voel mij hierin bevoorrecht.

Ik dank mijn promotor Prof. Dr. Maessen, hoogleraar cardiothoracale chirurgie. Beste Jos, jouw inzet en gedrevenheid om van de afdeling cardiothoracale chirurgie een "state of the art" product te maken op klinisch-, wetenschappelijk-, en onderwijsgebied, vind ik bewonderenswaardig. Je vakoverstijgende wetenschappelijke en klinische interesse werkt inspirerend. Dank voor het in mij gestelde vertrouwen om het "Short-Stay Intensive Care" project, een intensieve samenwerking tussen operatiekamer en intensive care en tussen de afdeling cardiothoracale chirurgie en cardio-anesthesie, tot een succesvol einde te brengen. Dank voor je persoonlijke vriendschap en je culinaire hoogstandjes.

Ik dank mijn afdelingshoofd en promotor Prof. Dr. van Kleef, hoogleraar anesthesiologie. Beste Marten, bedankt voor de geboden gelegenheid en het gestelde vertrouwen om binnen de afdeling anesthesiologie onderzoek te mogen en kunnen doen. Ik hoop deze leerzame periode in de toekomst te mogen voortzetten.

Het secretariaat anesthesiologie, Hanny Donders, Hermina Gielen, Marie-Josê Königs, Patrice Moonen-Florax, Sandra Reijnders-Keijdener, Thea Cremers, Wendy van Engelshoven - Thoma en Lilian van Thiel-Kraft, de centrale plaats van de afdeling waar iedereen altijd even vriendelijk wordt onthaald en er altijd tijd wordt gemaakt voor een kort persoonlijk en attent praatje, bedank ik voor de ondersteuning en belangstelling. 
Ik wil mijn collega cardio-anesthesiologen Chris Lawrence, Riet Smets, Frank Kwinten, John Gregoire (2007 †), Lex Pfaff (Maxima Medisch Centrum), Elise Machiels, Esther Bouwman, Jurgen Pétry, Henriëtte Willigers, Marcus Lancé en Jan-Willem de Poel bedanken voor de ruimte die ze me gaven om onderzoek te doen, hun flexibiliteit om weer eens van $\mathrm{OK}$ te ruilen om een onderzoekspatiënt te kunnen includeren en hun actieve inzet om het "Short-Stay Intensive Care" project te laten slagen.

Mijn collega Herman Bos, anesthesioloog/intensivist; Herman, bedankt voor je welgemeende interesse in de vorderingen van mijn promotieonderzoek en steun hiervoor.

De anesthesiemedewerkers, Bianca Marco, Aggie van der Sluijs Veer, Andrew Lotz, Angelica Smeets, Armand Beetz, Armand Ten Haaf, Bart Haemers, Bart Moesen, Bert Daemen, Bjorn Colen, Carla Roeffen, Centa Arentsen, Chrétien Muermans, Cornee Visser, Dalilah Leonora, Dennis Hebben, Desiree Cremer, Frank Ogg, Frans Theunissen, Gemma Haasdijk, Jacques van der Sluijs Veer, Jacqueline de Haan, Jeroen Hoebers, Jo Gielen, John Frissen, John Gerards, Kina Flaus, Laila Harti-Saba, Marc Jans, Marianne Cuypers, Marloes Homberg, Martine Vonk, Maud Speentjens, Marc Velmers, Marzenna van der togt-Zyutczynsk, Myra Monsewije, Nick Bedingfield, Peggy van der Maat, Roger Bekkers, Roy Pelzer, Ruurd van der Goot, Sandra Medenbach, Sanne Lindelauf, Simon Dixon, Sjaak Wojtowicz, Sylvia Wilhelmus, Wil Beckers, Ingrid Hollander-Mus, Jos Austen (PA), Paul Stocker, Wilbert Oudmans en oud medewerkers Jean Motten, Eugene Branche, Pierre van Engelshoven, Harry Janssen, Wim Nijssen, samen met jullie zorgen we voor het welzijn van de patiënt op de operatiekamer, wens ik van harte te danken voor hun professionele inzet, enthousiasme en plezierige samenwerking elke dag weer. Mijn bijzondere dank gaat ook naar Lion Stassen en René Roubben voor het warme hart dat ze de cardio-anesthesiologie toedragen, nooit was hen iets te veel om klinische zorg in combinatie met onderzoek mogelijk te maken.

De cardiochirurgen, Gijs Geskes, Prof. Dr. Bas Mochtar, Paul Barenbrug, Leen van Garsse, Mark La Meir, Ted Elenbaas (Catharina ziekenhuis Eindhoven) en Kees Prenger (Haga ziekenhuis Den Haag), de artsen Ceciel Soemers en Berry Phelps en de cardiologen Dr. Vincent van Ommen en Jindra Vainer, bedank ik voor de collegiale en plezierige samenwerking bij de de totstandkoming van het "Short-Stay Intensive Care" project. Het secretariaat van de cardiothoracale chirurgie, dank ik voor alle geboden ondersteuning voor mijn onderzoek, Lysette Liégeois-Bouvrie, Helma Aerdts-Poesen, Anja de Rooij-Klinkers, Lucienne Manders, Sylvia LebonFrederix.

Het verplegend personeel van de operatiekamers en in het bijzonder Patrick Biesmans, Tamara Broeksmit, Gaby Deumes, Barbara Dijk, Ramona Dingena, Aukje Edelbroek, Maya Sisic-Haurdic, Ilona Intemann, Miranda Janssen, MarieJose van de Camp, Kim Minkenberg, Marieke Eygelshoven, Shirly Fijttes-Brandt, Irmina Feyts-Bakkes, John Schreurs, Bert Hu-a-ng, Edith Claessens, Nora Harim- 
Oubaha, Stella Janssen-Scheerder, Brigitte Schevelier-Albus, Carina SluismansKuik, Martijne Tromp, Eef Brulmans-Wijnants en Diana Onstenk dank ik voor hun fijne samenwerking en hun niet-aflatende toewijding voor de patiënt om deze ook na afloop van de operatie optimaal te begeleiden tot aan het transport naar de intensive care.

De perfusionisten, Dick de Jong, Yvonne Bosch, Nicole Mak-Caberg, Diana van Dijk, Evelien Janssen-Vernaus, Dr. Yuri Ganuschak (ik heb nu weer tijd voor "only one question"), Ellen Heerdt, Georgette Molegraaf, Annekatrien Menten, Erwin Severdija, Marc Souren, Pieter Wortel, Dr. Patrick Weerwind en Jan de Munnik dank ik voor hun gewaardeerde samenwerking in de zorg voor onze patiënten en bij het het totstandkomen van de diverse onderzoeken.

Dr. Fransen, bewegingswetenschapper (Vitaphone Nederland, Tilburg); beste Erik, je hebt me geintroduceerd in de wereld van inflammatie en de diepere geheimen van de statistiek helpen exploreren, dank hiervoor.

Dr. Damoiseax, medisch immunoloog; beste Jan, bedankt voor de interessante en prettige samenwerking op het gebied van de cytokines.

Drs. Liem, anesthesioloog (Slingeland ziekenhuis, Doetinchem); beste Andy, bedankt voor je enthousiasme en gedrevenheid orn de studie "Pulmonary function and inflammatory markers in patients undergoing coronary revascularisation with or without cardiopulmonary bypass" tot een goed einde te brengen.

Dhr. Theunissen, onderzoekverpleegkundige; beste Maurice, dank voor je hulp bij het includeren van onderzoekspatiënten, je correcte werkwijze en de nauwkeurige dataverzameling.

Drs. van Mastrigt, bewegingswetenschapster (MAASTRO Clinic); beste Ghislaine, samen zijn we begonnen aan het "Short-Stay Intensive Care" project, na een voorzichtige start volgde een productieve samenwerking, jouw boekje is ook bijna klaar, heel veel succes en sterkte met de laatste loodjes. Dank je wel voor je fijne samenwerking.

Monique Ben-Miesen, physisian assistant cardiothoracale chirurgie, en Monique Jacobs-Dols, onderzoeksverpleegkundige, dank ik voor de nauwkeurige datavergaring van meer dan 600 onderzoekspatiënten, een hele klus.

Dr. Willigers, anesthesiologe; beste Henriëtte, partner in cardioanesthesieresearch, onderzoek doen komt niet vanzelf en gaat niet vanzelf, het vergt hard werken en doorzetten met een flinke portie geluk en humor, dank je voor je steun en luisterend oor om mijn beklag in ontvangst te nemen wanneer ik weer eens mijn research-dagje niet had.

Dr. van Hooren, psychologe en Universitair Docent Open Universiteit Nederland, beste Susan, dank voor al je 'tips and tricks' die je me gaf aangaande statistiek, epidemiologie en data-verwerking. Dank je wel dat je een van mijn paranimfen bent.

Dr. van der Hulst, plastisch chirurg; beste René dank je wel voor je lange vriendschap en dat je mijn paranimf wil zijn. 
De afdeling Cardiochirurgische Intensive Care, onder leiding van hoofdverpleegkundige C. Bout; beste Christophe, jouw leiderschapsstijl, variërend van participerend op de werkvloer tot gezag hebben en krijgen als afdelingshoofd, is voor mij nog steeds letterlijk voorbeeldig. De intensive-care verpleegkundigen Nicole Feij, Pim Ackerman, Kim Bartels, Ben Bouvrie, Guy Colson, Henk Creemers, Frans Creusen, Miet van Dael, Joep Dinjens, Monique Dols, Danny Donders, Rob Franssen, Rosanna Frau, Annick Groenen, Serge Heines, Peter Hendriks, Maurice Hoorens, Sonya Karmane, Gerda Kikkert, Paul Koeijmans, Vivianne Kuipers, Leon Moonen, Gerard Muller, Guus van der Nat, Janine Olivers, Jean Piere Partoens, Mieke Peeters, Francis Pieters, Johnan Raets, Wivina Rousseau, Maurice Scheres, Andre Steenhof, Marcella Terwingen, Jean Marie Thijs, Sjarel Verhoeven, Marion van der Vleuten, Leon Voncken, Marius van de Warenburg, Marc de Wilde, Cindy Cleuren, alsook de secretaresses Monique Drielsma en Jolanda Stessen, dank ik voor hun professionele en gemotiveerde inzet, de fijne samenwerking en voor alles wat ik van hun heb mogen leren. Jullie bijdrage, inzet en wil tot veranderen en verbeteren van zorgprocessen hebben van het "Short-Stay Intensive Care" project een succes gemakt.

Prof. Dr. Drs. Pieter, bewegingswetenschapper en niet-westers socioloog, Science University of Malaysia, Kubang Kerian, Kelantan, Malaysia; beste Willy, jouw enthousiasme voor kennisoverdracht, om vragen met vragen te beantwoorden en zo inzicht te laten groeien en om onderzoek te doen heeft vele jaren geleden een eerste kiem gelegd die nu een rijpe vrucht is geworden. Dank je hiervoor.

Verder wens ik iedereen te bedanken die betrokken is bij de zorg voor de hartchirurgische patiënt in het azM. Het is enkel door teamwork en een goede teamspirit dat we een zorglijn hebben kunnen opbouwen waar we met z'n allen trots op mogen zijn en die maximale garanties biedt voor een zo optimaal mogelijke zorg voor elke patiënt.

Tante Tinie Heijmans-Debije wil ik bedanken voor het mooie schilderij dat trots de kaft van mijn promotieboekje siert.

Toos Heijmans, mijn moeder, en mijn schoonouders, Noël en Margriet van Hooren, veel dank voor alles wat jullie voor mij gedaan hebben, vroeger en nu.

Mijn zoontjes Toine en Remi, er komt nu weer tijd vrij om met jullie door te brengen en het zal er ook ontspannender aan toe gaan, dat beloof ik.

Als allerlaatste, maar wel als allerbelangrijkste, dank ik mijn lieve vrouw Vivian. Schat, bedankt voor je begrip, je medeleven en je liefde. Je zag vanaf het begin dat het doen van onderzoek geen kortstondig gebeuren zou zijn, maar gaf me toch alle ruimte en steun.

Bedankt lieverd. 


\section{CURRICULUM VITAE}

John Heijmans werd op 6 februari 1960 geboren te Echt (Limburg). Na zijn middelbaar onderwijs aan het Bisschoppelijk College te Echt begon hij an de opleiding voor Leraar Lichamelijke Opvoeding aan de Katholieke Academie voor Lichamelijke Opvoeding (K.A.L.O.) te Tilburg (1979-1983). Hierna studeerde hij Bewegingswetenschappen (Inspanningsfysiologie) aan de afdeling Physical Education and Human Movement Studies van de University of Oregon, Eugene, Oregon, USA (1984-1986) waar hij zijn Master of Science (MS) behaalde. In 1997 startte hij zijn opleiding geneeskunde aan de Universiteit van Maastricht (1998 Propaedeutisch examen, 2000 Doctoraal examen, 2002 Artsexamen). Na een jaar werkzaam te zijn geweest op de eerste hulp afdeling van het St-Gregorius ziekenhuis te Brunssum en een jaar op de afdeling Cardiopulmonale chirurgie van het academisch ziekenhuis Maastricht, begon hij in 2000 aan zijn opleiding Anesthesiologie (2000-2005) met als opleider Prof. Dr. S. de Lange. Sindsdien is hij werkzaam als staf-anesthesioloog met als aandachtsgebied cardio-anesthesiologie, thoracoabdominale vaatchirurgie en research. Verder is hij lid van het implementatieproject "Short-Stay Intensive Care after Cardiac Surgery" en van de bloedtransfusie commissie van het azM.

John Heijmans was born in Echt, the Netherlands, on February six 1960. After finishing high-school at the Bisschoppelijk College in Echt, he studied Physical Education at the Catholic Academy for Physical Education (K.A.L.O.) in Tilburg (1979-1983). He studied at the department of Physical Education and Human Movement Studies at the University of Oregon, Eugene, Oregon, USA, were he received his Master of Science (MS) degree (1984-1986). In 1997 he started his medical studies at the University of Maastricht, Maastricht, the Netherlands (1997 2002). As a resident, he worked for one year at the First-Aid in the St-Gregorius hospital in Brunssum and one year at the department of Cardiothoracic surgery at the University Hospital Maastricht. He started his training as a resident in anesthesiology at the department of Anesthesiology of the University Hospital Maastricht (Head: Professor Dr. S. de Lange) (2000-2005) and continued working at the department of Anesthesiology as a staf-anesthesiologist with special interest in cardiac anesthesiology, thoracoabdominal vascular surgery and research. He is member of the "Short-Stay Intensive Care after Cardiac Surgery " clinical pathway development program and member of the blood transfusion committee of the University Hospital Maastricht. 


\section{PUBLICATIONS}

\section{ARTICLES}

Heijmans J.H., G.A.P.G. van Mastrigt, J.G. Maessen, and P.M.H.J. Roekaerts. Shortening ICU Stay After Coronary Artery Surgery. Submitted for publication.

Heijmans J.H., Y.M. Ganushak, M.S. Theunissen, J.G. Maessen, and P.M.H.J. Roekaerts. Assessment of Cardiac Responsiveness to Fluid Therapy After Cardiac Surgery. Accepted for publication in the Journal of Cardiothoracic and Vascular Surgery.

Willigers H., J. Heijmans, J. Maessen, and P. Roekaerts. Homeostatic Responses after Cardiac Surgery: Effect of Anaesthesia Management. Submitted for publication

Heijmans J. H., K.S.A.E. Liem, G.M.C. Damoiseaux, J.G. Maessen, and P.M.H.J. Roekaerts. Pulmonary Inflammation and Abnormalities After Coronary Revascularisation With or Without Cardiopulmonary Bypass. Anaesthesia 2007, doi: 10.1111/j.1365-2044.2007.05254.x

Heijmans J., E. Fransen, W. Buurman, J. Maessen, and Paul Roekaerts Comparison of the Modulatory Effect of Four Different Fast-Track Anesthetic Techniques on the Inflammatory Response to Cardiac Surgery with Cardiopulmonary Bypass. Journal Cardiothoracic and Vascular Anesthesia. Vol 21, No 4 (August), 2007: pp 512-518.

Mastrigt van G.A.P.G, J.G. Maessen, J.H. Heijmans, J.L. Severens and M.H. Prins. Does Fast-Track Treatment Lead to a Decrease of Intensive Care Unit and Hospital Length of Stay in Coronary Artery Bypass Patients? A Meta-Regression of Randomized Clinical Trials. Critical Care Medicine, 2006. Vol 34, No 6, p: 1-11.

Mastrigt van G.A.P.G., J.H. Heijmans, J.L. Severens, E.J. Fransen, P.M.H.J. Roekaerts, G. Vos and J.G. Maessen. Short-Stay Intensive Care After Coronary Artery Bypass Surgery: Randomized Clinical Trial on Safety and Cost-Effectiveness. Critical Care Medicine, 2006. Vol. 34, No 1, p: 65-75.

Heijmans J., Maessen J. and Roekaerts P. Remifentanil Provides Better Protection Against Noxious Stimuli During Cardiac Surgery than Alfentanil. European Journal of Anaesthesiology, 2004. Vol 21. p: 612-618. 
Heijmans J., Maessen J. and Roekaerts P. Risk Stratification for Adverse Outcome in Cardiac Surgery, Review. European Journal of Anaesthesiology, 2003. Vol 20. p: 515-527.

Heijmans J.H., Smeets H.G.W. and Janevski B.K. Leiomyosarcoma of the Inferior Vena Cava. A Case History. Vascular Surgery, 1995. Vol 29, No 5, p: 405-409.

Peeze Binkhorst F.M., Kuipers H., Heijmans J., Frederik P.M., Slaaf D.W., Tangelder G-J. and Reneman R.S. Exercise Induced Focal Skeletal Muscle Fiber Degeneration and Capillary Morphology. Journal of Applied Physiology, 1989. Vol 66, No 6, p: 2857-2865.

Pieter W., L.T. Bercades, and J. Heijmans. Injuries in Young and Adult Taekwondo Athletes. Kinesiology, 1998. Vol. 30, No 1: p 22-30.

Pieter W., G. van Ryssegem, R. Lufting, and J. Heijmans. Injury Situation and Injury Mechanism at the 1993 European Taekwondon Cup. Journal of Human Movement Studies, 1995. Vol. 28, no 1:p $1-24$.

Pieter W., D. Taaffe, R. Troxel, and J. Heijmans. Quadriceps/Hamstrings Ratios of Taekwondo Club Athletes and Beginning Tennis Players. Journal of Physical Education and Sport Sciences, 1992. Vol. IV, No 1: p 31-39.

Pieter W., E.D. Zemper, and J. Heijmans. Taekwondo Blessures. Geneeskunde en Sport, 1990. Vol. 23, No 6: p $222-228$.

Pieter w., D. Taaffe, and J. Heijmans. Heart Rate Response to Taekwondo Forms and Technique Combinations: A Pilot Study. The Journal of Sports Medicine and Physical Fitness, 1990. Vol. 30, no 1:p 97-102.

Pieter W., and J. Heijmans. Hamstring/Quadriceps Ratios en Piek Vermogen bij Toptaekwondoka. Nederlands Tijdschrift voor Fysiotherapie, 1989. Vol. 99, No 10: p $283-286$.

Heijmans J. en W. Pieter. Verschillen in Maximaal Moment van Mannelijke en Vrouwelijke Vechtsporters. Sportmedische Tijdingen, 1989. Vol. 11, No 41: p 95-101.

Pieter W., J. Heijmans, and D. Taaffe. Ioskinetic Leg Strength of Taekwondo Practitioners. Asian Journal of Physical Education, 1989. Vol. 12, No 3: p 57-64. 
Pieter W., D. Taaffe, R. Troxel, and J. Heijmans. Isokinetic Peak Torque of the Quadriceps and Hamstrings of College Age Taekwondo Athletes. Journal of Human Movement Studies, 1989. Vol. 16, No 1: p 17-25.

Pieter F. Pieter W., and J. Heijmans. Movement Analysis of Taekwondo Techniques. Asian Journal of Physical Education, 1987. Vol. 10, No 3: p 45-58.

Heijmans J.H. . A Comparison of Lower Extremity Strenght Patterns Between Young Gymnasts and Nonathletes (1986). Master's Thesis. Begeleider Prof. Dr. J. Broekhoff. University of Oregon, Eugene, Oregon, USA.

\section{ABSTRACTS}

Severdija E., J. Heijmans, G. Geskes, D. Jong, and J. Maessen. Reduced Heart Lung Machine Prime Reduce Blood Transfusion Requirements in Coronary Artery Surgery. SATS and SCANSECT abstract book congress proceedings, 016. August, 2006 Reykjavik, Iceland.

Heijmans J. H., A.K.S.E. Liem, J.G. Maessen and P.M.H.J. Roekaerts. OffPump Cardiac Surgery Causes Less Systemic Inflammation But This Does Not Translate Into Less Organ Dysfunction. European Journal of Anaesthesiology, 2006. Vol 23, Suppl 38, Abstract O-39: p 14.

Liem A.K.S.E., J.H. Heijmans, J.G.M.C. Damoiseaux, J.G. Maessen, and P.M.H.J. Roekaerts. Off-Pump Cardiac Surgery Does Not Reduce Inflammatory Mediator (IL-8 and TNF-a) Lung Measurements. Anesthesiology 2006; 105: A1021

Heijmans J., E. Fransen, J. Maessen, and P. Roekaerts. Effects of Anesthetic Technique on the Inflammatory Response to Cardiac Surgery. Nederlands Tijdschrift voor Anesthesiologie, 2006. Vol. 19, maart: p 31-32.

Roekaerts .P, J. Heijmans, G. van Mastrigt, J. Severens, E. Fransen, G. Voss, J. Maessen. Short-Stay Intensive Care na Coronairchirurgie: Een Veilige en KostenEffectieve Methode? Nederlands Tijdschrift voor Anesthesiologie. Vol. 17, Mei 2004, Supplement.

Garsse van L., J. Heijmans, M. de Baets, and J. Maessen. Robot Assisted Radical Thymectomy; First Clinical Experiences. Voordracht: Nederlandse Vereniging voor Thorax Chirurgie. Voorjaarsvergadering 22 april 2005, te Amsterdam. Netherlands Heart Journal 2005; Vol 13: 1: p 24. 
Roekaerts P.M.H.J., J.H. Heijmans, S.J.H. Heines, and J.J. Dinjens. Lung Recruitment After Cardiac Surgery: A Comparison of 2 different Alveolar Recruitment Strategies. Intensive Care Medicine. Volume 30, Supplement 1, September, 2004.

van Mastrigt G.A.P.G., J.L. Severens, P. Roekaerts, J. Heijmans J, E.J. Fransen and J.G. Maessen. Short Stay Intensive Care for Low Risk CABG-Patients: The New Standard Treatment for Postoperative Care of Low Risk CABG-Patients in the Netherlands? Netherlands Heart Journal. Volume 12. No 7/8, Augustus, 2004.

Fransen E.J., J.H. Heijmans, P.M.H.J. Roekaerts, W.A. Buurman and J.G. Maessen. The Impact of Anesthesia Regimen on the Release of Inflammatory Mediators and Catecholamines: A Prospective Randomized Trial in Cardiac Surgery Patients. Chest, 2002. Abstracts of Original Investigators. Vol 122, no 4 (suppl) October, p: 120S.

Peeze Binkhorst F.M., H. Kuipers, J. Heijmans, P.M. Frederik, D.W. Slaaf D and R.S. Reneman. Exercise Induced Capillary Changes in Skeletal Muscle. Ultramicroscopy, 1988. Vol 24, p: 445.

Pieter W., L.T. Bercades, and J. Heijmans. Competition Injuries in Olympic Taekwondo. In: Congress Proceedings fourth IOC World congress on Sport Sciences, 1997. Monte Carlo: International Olympic Committee, p: 117.

Pieter W., D. Taaffe, R. Troxel, and J. Heijmans. Isokinetic Strenght of Elite Taekwondo Athletes, Seoul Olympic Scientific Congress Organizing Committee (ed), Abstract. New Horizon of Human Movement, p 69. Seoul: Danhook University Cheonan Campus.

\section{ORAL PRESENTATIONS}

European Association of Cardiothoracic Anaesthesiologists (EACTA). Venice Mestre, Italy, May 24-27, 2006.

Title: Off-pump Versus Conventional Cardiac Surgery: Less Systemic Inflammation but Not Less Organ Dysfunction?

The Failing Heart Under Stress. Multifactorial Aspects of Acute and Chronic Heart Failure. Amsterdam, 20-22 November 2005.

Title: Effects of Anesthetic Technique on the Inflammatory Response to Cardiac Surgery.

2e Wetenschaps Dag Anesthesiologie. AMC, 30 September, 2005, Amsterdam. 
Title: Effects of Anesthetic Technique on the Inflammatory Response to Cardiac Surgery.

Nederlandse Verening Anesthesiologie (NVA). Anesthesiologen Dagen: Maastricht, 19-20 mei, 2005.

Title: Effects of Anesthetic Technique on the Inflammatory Response to Cardiac Surgery.

Nederlandse Verening Anesthesiologie (NVA). Anesthesiologen Dagen: Papendal, 13- 4 Mei, 2004.

Title: Short-Stay Intensive Care na Coronair Chirurgie: Een Veilige en KostenEffectieve Methode?

Symposium Doelmatiger Gezondheidszorg: Kleine Projecten, Grote Verbeteringen! (ZonMw) Vrije Universiteit Medisch Centrum te Amsterdam, 18 Juni 2004.

Title: Short-Stay Intensive Care na Coronair Chirurgie: Een Veilige en KostenEffectieve Methode?

European Association of Cardiothoracic Anesthesiologists (EACTA). Czech Republic, Prague, 25-28 May 2003.

Title: Remifentanil Versus Alfentanil and Target-Controlled Infusion of Propofol for Cardiac Anesthesia.

\section{POSTER PRESENTATIONS}

3e Wetenschaps Dag Anesthesiologie. AMC, 29 September 2006, Amsterdam.

Title: Off-Pump Versus Conventional Cardiac surgery: Less Systemic Inflammation but Not Less Lung Dysfunction?

3e Wetenschaps Dag Anesthesiologie. AMC, 29 September 2006, Amsterdam.

Title: Stroke Volume Variation is a Reliable Parameter for Preducting the Hemodynamic Response to a Fluid Load.

The Failing Heart Under Stress. Multifactorial Aspects of Acute and Chronic Heart Failure. Amsterdam, 20-22 November 2005.

Title: Effects of Anesthetic Technique on the Inflammatory Response to Cardiac Surgery.

European Society of Intensive Care Medicine (ESICM). Berlin, 10-13 October 2004. 
Title: Lung Recruitment After Cardiac surgery: A Comparison of Two Different Alveolar Recruitment Strategies.

\section{BOOKS}

Pieter W and Heijmans J. (1997, 2nd Edition 2000), Scientific Coaching of Competitive Taekwondo Athletes. Meyer \& Meyer Verlag, Aachen. (English translation of German version, Taekwondo. Technik, Training, Selbstverteidigung.).

Pieter W and Heijmans J. (1995), Taekwondo. Technik, Training, Selbstverteidigung. Meyer \& Meyer Verlag, Aachen.

Pieter W, en Heijmans J. (1989), Taekwondo (WTF) Stijlfiguren. De Vrieseborch, Haarlem. 